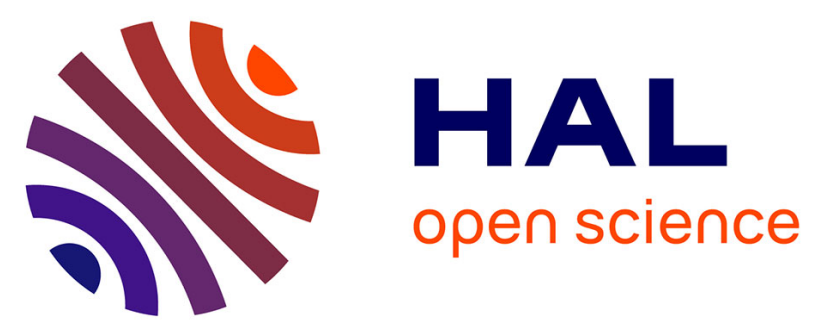

\title{
Proteomic investigation of phosphorylation sites in poly(ADP-ribose) polymerase-1 and poly(ADP-ribose) glycohydrolase.
}

Jean-Philippe Gagné, Xavier Moreel, Pierre Gagné, Yves Labelle, Arnaud Droit, Mélissa Chevalier-Paré, Sylvie Bourassa, Darin Mcdonald, Michael J. Hendzel, Claude Prigent, et al.

\section{To cite this version:}

Jean-Philippe Gagné, Xavier Moreel, Pierre Gagné, Yves Labelle, Arnaud Droit, et al.. Proteomic investigation of phosphorylation sites in poly(ADP-ribose) polymerase- 1 and poly(ADP-ribose) glycohydrolase.. Journal of Proteome Research, 2009, 8 (2), pp.1014-29. 10.1021/pr800810n . inserm00354464

\section{HAL Id: inserm-00354464 https://www.hal.inserm.fr/inserm-00354464}

Submitted on 21 Sep 2011

HAL is a multi-disciplinary open access archive for the deposit and dissemination of scientific research documents, whether they are published or not. The documents may come from teaching and research institutions in France or abroad, or from public or private research centers.
L'archive ouverte pluridisciplinaire HAL, est destinée au dépôt et à la diffusion de documents scientifiques de niveau recherche, publiés ou non, émanant des établissements d'enseignement et de recherche français ou étrangers, des laboratoires publics ou privés. 


\section{PROTEOMIC INVESTIGATION OF PHOSPHORYLATION SITES IN}

\section{POLY(ADP-RIBOSE) POLYMERASE-1 AND POLY(ADP-RIBOSE)}

\section{GLYCOHYDROLASE}

Jean-Philippe Gagné ${ }^{1,2}$, Xavier Moreel ${ }^{1}$, Pierre Gagné ${ }^{1}$, Yves Labelle ${ }^{1}$, Arnaud Droit ${ }^{1}$, Mélissa Chevalier-Paré ${ }^{1}$, Sylvie Bourassa ${ }^{3}$, Darin McDonald ${ }^{4}$, Michael J. Hendzel $^{4}$, Claude Prigent $^{2}$ and Guy G. Poirier ${ }^{1,3 *}$

${ }^{1}$ Laval University Medical Research Center, CHUQ, Faculty of Medicine, Laval University, 2705, Boulevard Laurier, G1V4G2, Québec, Canada

${ }^{2}$ CNRS UMR 6061 Institut de Génétique et Développement de Rennes, Université de Rennes 1, IFR140, 2 Avenue du Pr Léon Bernard, Rennes, France

${ }^{3}$ Proteomics Platform, Québec Genomic Center, Laval University Medical Research Center, CHUQ, Faculty of Medicine, Laval University, 2705, Boulevard Laurier, G1V4G2,

Québec, Canada

${ }^{4}$ Department of Oncology, University of Alberta and Cross Cancer Institute, Edmonton, Alberta, Canada T6G 1Z2

* Corresponding author

Guy.poirier@crchul.ulaval.ca

Tel: 418-654-2267

Fax: 418-654-2159

Keywords: PARP, PARG, Mass spectrometry, Phosphorylation 


\section{Summary}

Phosphorylation is a very common posttranslational modification event known to modulate a wide range of biological responses. Beyond the regulation of protein activity, the interrelation of phosphorylation with other posttranslational mechanisms is responsible for the control of diverse signaling pathways. Several observations suggest that phosphorylation of poly(ADP-ribose) polymerase-1 (PARP-1) regulates its activity. There is also accumulating evidence to suggest the establishment of phosphorylation-dependent assembly of PARP-1-associated multi-protein complexes. Although it is relatively straightforward to demonstrate phosphorylation of a defined target, identification of the actual amino acids involved still represents a technical challenge for many laboratories. Using a combination of bioinformatics-based predictions tools for generic and kinasespecific phosphorylation sites, in vitro phosphorylation assays and mass spectrometry analysis, we investigated the phosphorylation profile of PARP-1 and poly(ADP-ribose) glycohydrolase (PARG), two major enzymes responsible for poly(ADP-ribose) turnover. Mass spectrometry analysis revealed the phosphorylation of several serine/threonine residues within important regulatory domains and motifs of both enzymes. Using in vivo microirradiation-induced DNA damage, we show that altered phosphorylation at specific sites can modify the dynamics of assembly and disassembly of PARP-1 at sites of DNA damage. By documenting and annotating a collection of known and newly identified phosphorylation sites, this targeted proteomics study significantly advances our understanding of the roles of phosphorylation in the regulation of PARP-1 and PARG. 


\section{Introduction}

Poly(ADP-ribose) polymerase-1 (PARP-1) is an enzyme that catalyzes the hydrolysis of NAD and the enzymatic transfer of multiple ADP-ribose units to attach poly(ADP-ribose) ( $\mathrm{pADPr}$ ) on numerous target proteins, including PARP-1 itself. PARP-1 plays a role in major processes involving genomic integrity ${ }^{1}$ such as the detection and repair of DNA damage $^{2}$, chromatin modification ${ }^{3}$ as well as replication and transcription mechanisms ${ }^{4}$. In addition, PARP-1 is involved in cell death signaling pathways ${ }^{5,6}$ making it an attractive candidate for potential cancer therapies. The pADPr itself has been shown to be an apoptogenic molecule ${ }^{7,8}$. The rapid turnover of pADPr is mediated by poly(ADP-ribose) glycohydrolase (PARG), the major cellular pADPr catabolizing enzyme. Although PARP-1 and PARG have been mostly studied in a context of DNA-damage, chromatin-associated functions and apoptosis, there is accumulating evidence that pADPr metabolism is important in normal cellular physiology ${ }^{9-11}$.

Many studies suggest that PARP-1 activity may be regulated by phosphorylation involving several kinases that are parts of important regulatory pathways. Initial in vitro experiments indicated that phosphorylation of PARP-1 by protein kinase C (PKC) inhibits its DNAbinding and polymerase activities ${ }^{12,13}$. Subsequently, in vivo data revealed that PARP-1 is phosphorylated following phytohemagglutinin stimulation of resting lymphocytes ${ }^{14}$ and during Xenopus laevis oocyte maturation ${ }^{15}$. More recently, Beckert and collaborators ${ }^{16}$ showed that treatment of endothelial cells with IGF-I resulted in an inhibition of PARP-1 activity concomitantly with its phosphorylation. Because activation of the extracellular 
signal-regulated kinases 1 and 2 (Erk1/2) pathway is a major effector in response to IGF-I binding its receptor ${ }^{17}$, it is tempting to speculate that the phosphorylation of PARP-1 by Erk is responsible for the decrease in PARP-1 activity. However, a report from Kauppinen et al. ${ }^{18}$ appears to be in conflict with this hypothesis as the authors observed that the phosphorylation of PARP-1 by Erk1/2 is required for its full activation following treatment with the alkylating agent N-methyl-N'-nitro-N-nitrosoguanidine (MNNG). DNAindependent PARP-1 activation can also be triggered by the direct interaction of PARP-1 with phosphorylated Erk-2, an intriguing phenomenon in which PARP-1 is activated by a phosphorylated kinase without being phosphorylated itself ${ }^{19}$. There is still some confusion in the literature with respect to the role of ERK on PARP activation. For example, a recent study from Ethier et al. ${ }^{20}$ demonstrated that pADPr synthesis following MNNG-induced genotoxicity is unchanged in HeLa cells upon indirectly inhibiting ERK by treating cells with a specific inhibitor of its upstream regulator MEK. Another PARP-1 mediated mitogen-activated protein kinase pathway (MAPK) was implicated in the contribution of the c-Jun-N-terminal kinase 1 (JNK1) to PARP-1 activation in response to $\mathrm{H}_{2} \mathrm{O}_{2}$-induced cell death ${ }^{21}$. In vitro phosphorylation assays have notably shown that PARP-1 is a direct phosphorylation target of JNK1.

Other kinases such as the calmodulin kinase (CaMK) have been postulated to regulate PARP-1 activity. PARP-1 and calmodulin pathways were found to be targeted by sulphur mustard-induced apoptosis ${ }^{22}$. The demonstration of PARP-1 activation by CaMK-II phosphorylation was reported by $\mathrm{Ju}$ et $a l .{ }^{23}$ in a model neurogenic gene activation pathway. Recently, membrane depolarization mediated by CaMK-II has been shown to induce 
dissociation of the PARP-1 repressor KIF4 from PARP-1 through CaMK-II-dependent phosphorylation of PARP-1 which results in an activation of PARP-1 ${ }^{24}$.

Recent observations have led to the view that PARP-1 signalling and involvement in DNAdamage response could also be mediated by its phosphorylation. Indeed, dynamic changes in protein-protein interactions regulating the assembly of multiprotein complexes are mediated by the specific phosphorylation of some members of these complexes. For example, phosphorylation of PARP-1 is required for the association with Txk, a member of the tec family of tyrosine kinases ${ }^{25}$.

Casein kinase 2 (CKII) is involved in the phosphorylation and regulation of several DNAdamage related proteins such as $\mathrm{p} 53^{26-28}, \mathrm{BRCA}^{29}, \mathrm{XRCC}^{30,31}$ and RNA components of the translational apparatus ${ }^{32}$, thus making this kinase a potentially interesting effector of PARP-1 mediated DNA-damage signalling pathway. One of the earliest reports suggesting that the cell's response to DNA damage is modulated by CKII-dependent protein phosphorylation is the work of Teitz et al. (1990) ${ }^{33}$ in which authors reported that a Xeroderma pigmentosum cell line exhibits increased resistance to UV irradiation when transfected with a cDNA encoding a CKII subunit. Later, a defective CKII has been identified as a gene that affects cells' ability to adapt to the yeast's DNA damage checkpoint ${ }^{34}$. In addition, the DNA-binding functions of $\mathrm{p} 53$ following UV radiationinduced DNA damage have been shown to be activated by phosphorylation of CKII sites ${ }^{28,}$ 35, 36 . The DNA damage response also involves the repression of genes whose products are components of the translational machinery ${ }^{32}$. Indeed, CKII has been proposed as the 
terminal effector in a signalling pathway that represses pol III transcription when genome integrity is compromised ${ }^{37}$. The unanticipated role for CKII in the control of DNA-damage response (reviewed in ${ }^{31}$ ) has been further supported by recent work from Loizou et al. ${ }^{30}$ in which CKII phosphorylation of XRCC1 has been demonstrated to be required for assembly and activity of DNA single-strand break repair protein complexes. Moreover, the association of the novel DNA end processing factor APFL (aprataxin- and PNK-like factor) with XRCC1, which results in a recruitment of DNA single- and double-strand break repair machinery, requires CKII-specific phosphorylation ${ }^{38,39}$. A role for CKII in double-strand break re-joining is also proposed via the phosphorylation of histone $\mathrm{H} 4$ in nucleosomes proximal to the double-strand break. This phosphorylation is required for the maintenance of genome integrity and may reflect the modified histones functioning as binding sites for various DNA repair proteins ${ }^{40}$.

Finally, a link between PARP-1 and the cyclin-dependent kinase 5 (CDK5) has been proposed since silencing this kinase strongly sensitize cells deficient in homologous recombination to PARP inhibitors ${ }^{41}$. Notably, CDK5 has been shown to be required for the DNA-damage response ${ }^{41,42}$. PARP-1 is also suspected to be a substrate of the integrinlinked kinase (ILK) ${ }^{43-45}$ and has been shown to be phosphorylated by the AMP-activated protein kinase (AMP-K) ${ }^{46}$, resulting in a marked increase in PARP-1 activity. Thus, there is a compelling body of evidence converging towards a complex PARP-1 phosphorylation model involving multiple kinases with different consequences on PARP activity. 
Collectively, these data indicate that phosphorylation is likely to play a significant role in the regulation of PARP-1 activity through both direct catalytic regulation and the regulation of the assembly of PARP-1 complexes. Because diverse signalling kinases may phosphorylate PARP-1, there is a potential for complex and dynamic regulation of PARP-1 in response to changing biological conditions. To date, there are no reports indicating that PARG can be regulated by phosphorylation although endogenous phosphorylation sites were detected by large-scale mass spectrometry analysis of phosphoproteomes ${ }^{47-50}$.

This study has been undertaken to gain further insight into the phosphorylation status of PARP-1 and PARG with potential relevance to multiple biological functions. Phosphorylated PARP-1 and PARG were digested with trypsin and analyzed by mass spectrometry. Several phosphorylated amino acid residues were identified in both enzymes. The phosphorylation sites were further analyzed as potential phosphorylation sites using an in silico method employing neural network predictions for serine, threonine and tyrosine phosphorylation sites in eukaryotic proteins (NetPhos 2.0) ${ }^{51}$ and predictions of kinasespecific eukaryotic protein phosphorylation sites (NetPhosK $1.0{ }^{52}$ and Phoscan ${ }^{53}$ ). Many PARP-1 phosphorylation sites are evolutionarily conserved and located within important functional domains, consistent with regulatory roles in vivo. Most intriguing is the clustering of PARG phosphorylation sites within the N-terminal putative regulatory domain. This suggests a confined and targeted phosphorylation pattern within the noncatalytic domain with potentially important regulatory effects on localization signals, caspase cleavage sites and putative protein-protein interaction motifs. 


\section{Materials and methods}

3.1 In silico prediction of PARP-1 and PARG phosphorylation sites

FASTA formatted human PARP-1 and PARG amino acid sequences (Swiss-Prot accession numbers, PARP-1: P09874, PARG: Q86W56) were submitted to NetPhos 2.0 server (http://www.cbs.dtu.dk/services/NetPhos/) to generate neural network predictions for serine, threonine and tyrosine phosphorylation potential. A generic prediction score classification was applied to the dataset for the phosphoresidue prediction: low: 0 to 0.499 , high 0.500 to 0.999 . Kinase-specific protein phosphorylation site prediction employing ESS filtering (Evolutionary stable sites) on the NetPhosK 1.0 server (http://www.cbs.dtu.dk/services/NetPhosK/) was also performed using the same sequence (threshold $=0.5)$. NetPhosK prediction of kinase-specific eukaryotic protein phosphorylation sites was performed with a dataset covering the following kinases: protein kinase A (PKA), protein kinase C (PKC), protein kinase G (PKG), casein Kinase II (CKII), cell division cycle 2 kinase (CDC2), calcium/calmodulin-dependent protein kinase type II (CaMK-II), serine-protein kinase Ataxia telangiectasia mutated (ATM), DNA-dependent protein kinase (DNA-PK), cyclin-dependent kinase 5 (CDK5), mitogen-activated protein kinase p38 (p38MAPK), glycogen synthase kinase 3 (GSK3), casein kinase I (CKI), protein kinase B (PKB), ribosomal S6 kinase (S6K), insulin receptor kinase (INSR), epidermal growth factor receptor (EGFR) and Src tyrosine kinase (SRC). These predictions were also corroborated by a Phoscan prediction of PARP-1 and PARG phosphorylation sites (http://bioinfo.au.tsinghua.edu.cn/phoscan/) using the following kinases with low 
stringency lod-odds ratios: PKA, cyclin-dependent kinase (CDK), CKII, PKC, protein kinase B (PKB), mitogen-activated protein kinase (MAPK) and ATM. Predictions with consensus sequences were generated from the following kinase dataset: Myosin light chain kinase (MLCK), cGMP-dependent protein kinase (PKG), S6K, CaMK-I, CaMK-II, AMPactivated protein kinase (AMP-K), extracellular signal-regulated kinase (ERK), Polo-like kinase 1 (PLK1), RNA-dependent protein kinase (PKR), p21-activated kinase (PAK), Abelson tyrosine kinase (Abl), c-terminal Src protein kinase (CSK), epidermal growth factor receptor (EGFR), fibroblast growth factor receptor (FGFR), insulin receptor (IR), focal adhesion kinase (FAK), TGF-beta receptor (TGFR), transforming growth factor beta receptor protein kinase (TGFbeta receptor PK), MAP kinase activated protein kinase 1 (MAPKAP-1), MAPKAP-2, never in mitosis protein kinase (NIMA) and DNA-PK.

\subsection{Recombinant PARP-1 and PARG}

Purified human PARP-1 expressed in SF-9 insect cell line was obtained from Alexis Biochemicals (San Diego, USA). Recombinant PARG was expressed and purified essentially according to Invitrogen and Qiagen's recommendations. E. coli BL21-AI ${ }^{\mathrm{TM}}$ (Invitrogen, Carlsbad, U.S.A.) transformed with OmicsLink ${ }^{\mathrm{TM}}$ (Genecopoeia Inc, Germantown, U.S.A.) vector (equivalent of Invitrogen's pDEST17 (T7 promoter, N-His tag)) expressing full length $6 \mathrm{X}$-His tagged human PARG protein was grown with shaking to an $\mathrm{OD}_{600}$ of 0.6 in a $37^{\circ} \mathrm{C}$ incubator, followed by the addition of $0.2 \% \mathrm{~L}$-arabinose. Cells were harvested 120 min post induction by centrifugation and suspended in non-denaturing 
lysis buffer (50 mM Tris- $\mathrm{HCl} \mathrm{pH}$ 8.0, $300 \mathrm{mM} \mathrm{NaCl}, 10 \mathrm{mM}$ imidazole and supplemented with Complete ${ }^{\mathrm{TM}}$ antiprotease (Roche Applied Science, Indianapolis, U.S.A.)) according to the manufacturer's instructions. Cells were disrupted using five 10-second bursts at 200$300 \mathrm{~W}$ with a 10-second cooling period between each burst. The lysate was then centrifuged at $10,000 \mathrm{xg}$ for $10 \mathrm{~min}$ at $4^{\circ} \mathrm{C}$ to pellet cellular debris. The supernatant was applied to $5 \mathrm{ml} \mathrm{Ni-NTA}$ agarose beads (Qiagen) pre-washed with lysis buffer and mixed gently for $60 \mathrm{~min}$ at $4^{\circ} \mathrm{C}$. The beads were washed three times with $30 \mathrm{ml}$ of lysis buffer containing $20 \mathrm{mM}$ imidazole. 6X-His tagged PARG was eluted from the beads using lysis buffer containing $250 \mathrm{mM}$ imidazole.

\subsection{In vitro phosphorylation assays}

Kinases used for PARP-1 phosphorylation assays were obtained from Upstate Biotechnology (Charlottesville, USA) [Erk1, Erk2, CaMK-II, PKC $\alpha$ and PKC $\beta$ ], New England Biolabs (Ipswich, USA) [CKII] and SignalChem (Richmond, BC, Canada) [JNK1 and CDK5]. Phosphorylation reactions were carried out as recommended by the manufacturers in the presence of either cold or $\left[\gamma_{-}^{32} \mathrm{P}\right] \mathrm{ATP}$. One microgram of recombinant PARP-1 was used in each phosphorylation reaction. Plasmin was obtained from SigmaAldrich Corp. (St. Louis, USA), and $2 \mu \mathrm{g}$ of PARP-1 was digested with $35 \mathrm{ng}$ of plasmin for $30 \mathrm{~min}$ at $30^{\circ} \mathrm{C}$ in phosphorylation buffer. Recombinant PARG $(2 \mu \mathrm{g})$ was phosphorylated with 500 units of CKII (New England Biolabs, Ipswitch, U.S.A.) according to the supplier's instructions. ${ }^{32} \mathrm{P}$-labeled PARP-1 and PARG were resolved by SDS- 
PAGE. After drying the gel, the radiolabeled proteins were visualized and quantified with an Instant Imager (Perkin-Elmer, Waltham, U.S.A), while unlabeled products were separated by SDS-PAGE and stained with Coomassie blue.

3.4 Sample preparation of in vitro phosphorylated PARP-1 and PARG for MS analysis

PARP-1 and PARG protein bands were manually excised from a SDS-PAGE gel and reduced with $10 \mathrm{mM}$ dithiothreitol (Sigma-Aldrich, St-Louis, U.S.A.) for 30 minutes at $37^{\circ} \mathrm{C}$ and then alkylated with $55 \mathrm{mM}$ iodoacetamide (Sigma-Aldrich) for 30 minutes. In-gel protein digestion was performed overnight with $250 \mathrm{ng}$ of mass spectrometry-grade trypsin (Promega, Madison, WI) at $37^{\circ} \mathrm{C}$. Peptides were extracted from the gel using a $50 \%$ acetonitrile:water solution and dried out with a vacuum dryer (SpeedVac, Savant instrument, Holbrook, NY).

\subsection{LC-MS/MS Analysis}

Dried peptides were resuspended in $4 \mu 1$ of $0.1 \%$ formic acid solution. On-line liquid chromatography of $2 \mu \mathrm{L}$ of the resuspended sample was performed using a Thermo MicroAS autosampler and Surveyor MS pump (Thermo Electron, San Jose, U.S.A.). Chromatographic separation was achieved on a PicoFrit column BioBasic C18, $10 \mathrm{~cm}$ x 75 $\mu \mathrm{m}$ (New Objective, Woburn, MA) with a linear gradient from $2 \%$ to $50 \%$ solvent B (acetonitrile, $0.1 \%$ formic acid) against solvent $\mathrm{A}$ (water, $0.1 \%$ formic acid) in 30 minutes, at $200 \mathrm{~nL} / \mathrm{min}$. The procedure for the analyses performed at the Taplin Facility (Harvard 
Medical School, Boston, U.S.A.) was the following. A nano-scale reverse-phase HPLC capillary column was created by packing $5 \mu \mathrm{m} \mathrm{C} 18$ spherical silica beads into a fused silica capillary $(75 \mu \mathrm{m}$ inner diameter x $12 \mathrm{~cm})$ with a flame-drawn tip. After equilibrating the column each sample was pressure-loaded off-line onto the column, and the column was then reattached to the HPLC system. A gradient was formed and peptides were eluted with increasing concentrations of solvent B (97.5\% acetonitrile, $0.1 \%$ formic acid).

Peptides eluted through the column directly into a LTQ linear ion trap mass spectrometer (Thermo Electron). Mass spectra were acquired using a data-dependent acquisition mode in which each full-scan mass spectrum (400 to $2000 \mathrm{~m} / \mathrm{z}$ ) was followed by collision-induced dissociation of the seven most-intense ions. The dynamic exclusion function was enabled, and the relative collisional fragmentation energy was set to $35 \%$.

3.6 Enrichment of phosphopeptides from endogenously phosphorylated PARP-1

A dried peptide extract obtained from a tryptic digestion of $4 \mu \mathrm{g}$ of recombinant PARP-1 was enriched for phosphopeptides with $\mathrm{TiO}_{2}$ mono-tip from GL science (Tokyo, Japan). The $\mathrm{TiO}_{2}$ resin was preconditioned with $100 \%$ acetonitrile $(\mathrm{ACN})$ and then conditioned with $0.2 \mathrm{M}$ phosphate buffer $\mathrm{pH}$ 7.0. Peptides were reconstituted in $100 \mu$ l loading solution (350 mg/ml 2,5-dihydroxybenzoïc acid in 80\% ACN and $0.1 \%$ trifluoroacetic acid (TFA)) and loaded on the tip. The $\mathrm{TiO}_{2}$ resin was washed with the loading solution followed by a second washing step with $80 \%$ ACN and $0.1 \%$ TFA. Phosphopeptides were eluted with 400 
$\mathrm{mM} \mathrm{NH}_{4} \mathrm{OH}$ and evaporated in a vacuum dryer (SpeedVac, Savant instrument, Holbrook, NY).

\subsection{MALDI MS analysis}

MALDI mass spectrometry was performed using both classical crystalline matrices and ionic liquid matrices (ILM). Classical 2,5-dihydroxybenzoic acid (DHB) (Sigma-Aldrich Corp) matrix was prepared in $50 \%$ acetonitrile/water to final concentration of $150 \mathrm{mM}$ while for ILM, $300 \mathrm{mM}$ of DHB and $300 \mathrm{mM}$ of n-Butylamine (BuA) (Fluka) were prepared in $50 \%$ acetonitrile/water to final concentration of $150 \mathrm{mM}$. Analyses were performed using a MALDI quadrupole time-of-flight (QqTOF) mass spectrometer (QSTAR-XL, MDS Sciex / Applied Biosystems) equipped with an OMALDI 2 highrepetition rate laser. Measurements were performed in both positive and negative ion mode. Data was collected using the Analyst QS 1.1 software and oMALDI server (MDS Sciex / Applied Biosystems). External mass calibration was achieved using standard peptides. Measurements using DHB were performed by searching for 5-10 hot spots, accumulating 100 shots in total. All measurements were repeated in at least two independent experiments.

\subsection{Interpretation of MS/MS Spectra}

MS/MS spectra were first processed with Sequest using a two-pass approach, similar in design and implementation to the second, "refinement" pass of TheGPM's search engine

(http://www.thegpm.org) ${ }^{54}$. The first pass was used to identify which proteins were present 
in the sample whereas the second pass maximized the number of correctly identified phosphopeptides. For both passes, tandem mass spectra were extracted from the LTQ raw files by BioWorks version 3.2 and analyzed using Sequest (Thermo Scientific, San Jose, CA; version 27 rev. 12), with a fragment ion mass tolerance of $1.0 \mathrm{Da}$ and a parent ion tolerance of 2.0 $\mathrm{Da}$. The first pass reasonably assumed the existence of two detectable and minimally modified tryptic peptides for each protein present in the LC-MS/MS sample. For the first pass, Sequest was set up to search the International Protein Index's human database (version 3.30) ${ }^{55}$ supplemented with a few common contaminants. The only fixed and variable modifications specified were respectively cysteine carbamidomethylation and methionine oxidation. Scaffold (version Scaffold_2_01_01, Proteome Software Inc., Portland, OR) was used to validate MS/MS-based peptide and protein identifications. Peptide identifications required deltaCn scores greater than 0.10 and XCorr scores greater than $1.8,2.5$, and 3.5 for singly, doubly, and triply charged peptides respectively. Protein identifications were accepted if they contained at least 2 identified peptides. For the second pass, each biological sample was processed separately. This time, Sequest was set up to search a sample-specific protein sequence database containing only those proteins identified in the sample during the first pass. Tryptic digestion with a maximum of 3 internal missed cleavages was assumed. Cysteine carbamidomethylation was specified as a fixed modification whereas oxidation of methionine and phosphorylation of serine, threonine and tyrosine were specified as variable modifications.

Scaffold (Proteome Software) was used to filter out second-pass peptide spectral matches (PSMs) with XCorr scores lower than 2.0 and annotate the fragments of the remaining 
MS/MS spectra. Every PSM corresponding to a possible PARP-1 or PARG phosphopeptide was manually validated for a high-quality match and, in the case of phosphoserine/threonine-containing peptides, for a strong MS/MS fragment corresponding to the neutral loss of phosphate from the precursor. Finally, when many possible phosphorylation sites existed within the identified phosphopeptide, the Ascore from Beausoleil et al. (2006) ${ }^{49}$ was computed using the authors' server (http://ascore.med.harvard.edu) in order to determine whether or not the phosphorylation could be confidently assigned to a specific residue. The Ascore is a probability-based score which measures the probability of correct phosphorylation site localization based on the presence and intensity of site-determining ions in the phosphopeptide MS/MS spectrum.

\subsection{Plasmid constructs and mutagenesis}

Plasmids used for GFP-tagged PARP-1 expression were all based on the constructs described previously ${ }^{56,57}$. Point mutations were introduced by site-directed mutagenesis (QuikChange mutagenesis kit (Stratagene)). Successive mutagenesis rounds were used to produce S27-S32-S41 triple mutants. The sequences of primers used for the various mutants are: S27D [5-CGTTCTTTACGTCGCTCCTGTAGGGGTTCCTGAGC-3], S32E [5-CGTAGGGGTTCCTGCTCGAGGCCTACCGGTAGTACC-3] and S41E [5CCTACCGGTAATACCACGTCCTCGGGTACAAACTACC-3]. In order to prevent hairpin loops, a silencing mutation was introduced at the codon corresponding to I37. 


\subsection{Two-photon micro irradiation}

The evaluation of recruitment kinetics of PARP-1 phosphomutants was performed essentially as described ${ }^{56}$ with the exception of the following modifications. After overnight transfections with Effectene reagent (Qiagen), HeLa cells were placed in medium containing $1 \mu \mathrm{g} / \mathrm{ml}$ Hoechst 33258 for 30 minutes. The medium was then removed and replaced with fresh one. A $37^{\circ} \mathrm{C}$ pre-heated stage was used for the acquisition period on the Zeiss LSM510 NLO laser-scanning confocal microscope. Because of the rapid accumulation but longer duration of accumulation of PARP-1 at sites of DNA damage, multiple acquistion rates were used. For the 30 first seconds, 20 images were collected every $250 \mathrm{~ms}$ followed by 10 images for $500 \mathrm{~ms}$ and 10 images for $2 \mathrm{~s}$. The following 20 pictures were collected for $36 \mathrm{~s}$ for a total of 60 images over $12.5 \mathrm{~min}$. Background and photobleaching corrections were applied to each datasets as described ${ }^{56}$.

\section{Results and discussion}

\subsection{In silico prediction of PARP-1 generic phosphorylation sites}

Prediction of phosphorylation sites were generated using NetPhos ${ }^{51}$ and Phoscan ${ }^{53}$ servers, which are both integrated systems for accurately predicting the location of phosphorylation sites. The first approach lies in the estimation of the phosphorylation potential of PARP-1 and PARG using $\mathrm{S} / \mathrm{T} / \mathrm{Y}$ "sequence logos" that represent the sequence context at experimentally verified phosphorylation sites. The NetPhos algorithm calculates a 
probability score for a phosphorylation at a given position. This computational prediction approach generates a generic score which is correlated to the likelihood of a phosphorylation without specificity determinants for a single kinase. This prediction approach is a valuable tool to pinpoint protein areas where phosphorylation has a high potential to occur and can assist in the determination of functionally important regulatory domains within a protein. Using this strategy, PARP-1 and PARG were subjected to sequence-based phosphorylation site prediction. The phosphorylation potential is depicted by a color-coded $\mathrm{S} / \mathrm{T} / \mathrm{Y}$ bar graph with respect to each protein amino acid sequence (Figure 1A and 1B, see Supplementary Table S1 for detailed prediction scores and complete PARP1 and PARG Serine/Threonine/Tyrosine (S/T/Y) coverage). Putative highly phosphorylatable PARP-1 residues are dispersed throughout the amino acid sequence but specific regions appear more likely to be phosphorylated (Fig. 1A). Notably, the first Nterminal zinc finger domains and the region centered around the BRCT domain have higher phosphorylation potentials. In contrast, the phosphorylation potential is remarkably low in the PARP signature motif that comprises the active site. The consequences of phosphorylated residues in functional and regulatory domains of PARP-1 are poorly understood but the identification of potential phosphorylation sites in specific areas can help in the design of targeted mutagenesis experiments. The increased susceptibility of phosphorylation of important motifs can also be taken into account when designing functional studies. 


\subsection{In silico prediction of PARG generic phosphorylation sites}

In contrast to PARP-1, which displays a dispersed putative phosphorylation pattern throughout most of its amino acid sequence, PARG most probable phosphorylation sites are clustered in the N-terminal part of the protein comprised of the first four exons (Fig 1B). This clustering of predicted phosphorylation sites is even more dramatic in the region surrounding the two caspase-3 cleavage sites. A closer analysis of this particular region reveals that the amino acid composition makes it an acidic region, which contrasts with the basic charge of the catalytic domain ${ }^{58}$. This acidic region is highly enriched in consensus CKII phosphorylation sites (Supplementary Table S1). Interestingly, a characteristic pattern of CKII phosphorylation in acidic regions has been reported for several proteins ${ }^{59-63}$, making CKII a prototypic acidic-directed kinase.

The preferential localization of putative phosphorylation sites within PARG N-terminal region suggests that this posttranslational modification might be important in the regulation of PARG activity. However, the N-terminal part of PARG, to which has been assigned a 45 $\mathrm{kDa}$ putative regulatory domain, remains to be characterized with respect to the regulation of PARG catalytic activity. Nonetheless, subcellular localization and susceptibility to caspase cleavage are a consequence of specific signals located within this N-terminal region. 
4.3 Prediction of specific kinase phosphorylation sites in PARP-1 and PARG

NetPhosK and Phoscan were also used to predict PARP-1 and PARG kinase-specific phosphorylation sites from PARP-1 and PARG (Suplementary Table S2) as these predictions help interpret mass spectrometric results, namely why some sites appear to be phosphorylated only by a specific kinase. NetPhosK and Phoscan use different methods to generate kinase-specific predictions. NetPhosK is based on a machine learning technique called "artificial neural networks" that computes a probability score accounting for the sequence variations around the kinases acceptor sites. NetPhosK also examines homologous phosphorylation sites in related species to improve prediction reliability (evolutionary stable sites filtering). For each putative S/T/Y phosphoresidues, NetPhosK estimates which kinase is more likely to find its substrate given the kinase's general phosphorylation pattern. Kinase-specific phosphorylation modelling has been further developed by Li et al. ${ }^{53}$ with the Phoscan program. Phoscan integrates biological information regarding amino acid composition in the neighbourhood of phosphorylation sites and kinase-specific features for recognizing substrates. Phoscan integrates phosphorylation potential predictions of candidate sites based on their amino acid composition to kinase-specific features for recognizing substrates as reported in the literature, resulting in a more accurate prediction. 
4.4 Mass spectrometry analysis of PARP-1 and PARG phosphoresidues

We were inspired to map PARP-1 and PARG kinase-specific phosphorylation sites by mass spectrometry for two reasons. First, although in silico prediction of phosphorylated residues within PARP-1 and PARG sequences is a remarkable tool to designate residue candidates for functional analysis, experimental validation of a given phosphorylation site increases the probability that it could be functionally relevant. Secondly, while some kinases are known to phosphorylate PARP-1, there is currently very little information in the literature in relation to the exact phosphorylation sites. Kinase-specific mapping was accomplished by subjecting recombinant PARP-1 to phosphorylation by kinases suspected to be involved in PARP-1-dependent pathways such as PKC, Erk-1/2, CaMK-II, JNK1 and CDK5. CKII phosphorylation sites were investigated in both PARP-1 and PARG since there is accumulating evidence of CKII as a key participant in the cellular response to DNA

damage $^{31}$. Moreover, the identification of CKII phosphorylation sites in PARG is particularly interesting seeing that predicted CKII phosphorylation sites are abundant in the N-terminal PARG regulatory region (Supplementary Table S1).

\subsubsection{PARP-1 phosphomapping}

Considering the initial reports of phosphorylation of PARP-1 by PKC ${ }^{12,13,64}$ and the overrepresentation of predicted PCK phosphorylation sites in PARP-1 (about one third of 
all predicted sites (Supplementary Table S1)), we first incubated human PARP-1 with PKC $\alpha$ and $\beta$ in the presence of $\left[\gamma^{32} \mathrm{P}\right] \mathrm{ATP}$. In both cases, we observed strong phosphorylation of PARP-1 (Fig. 2A). Since the signal was stronger with PKC $\beta$, we used this kinase to phosphorylate PARP-1 with unlabeled ATP and perform LC-MS/MS on the trypsindigested, phosphorylated PARP-1. Three phosphopeptides were identified, two containing a phosphoserine $\left(\mathrm{S}^{504}\right.$ and $\mathrm{S}^{519}$ ) and one containing a phosphothreonine $\left(\mathrm{T}^{656}\right)$. Table 1 lists all the phosphopeptides and the phosphorylated residues identified by mass spectrometry. SEQUEST Xcorr and deltaCn scores, which are indicative of the quality of the peptidespectral match are presented for each phosphopeptide identified by MS/MS.

Post-translational modifications such as phosphorylation can be observed by mass spectrometry since they shift, by a characteristic value, the mass of the modified peptide and of all its collision-induced fragments bearing the modification. For instance, phosphorylation increases the mass of the peptide and of the MS/MS fragments bearing the phosphate by $79.97 \mathrm{Da}$. Furthermore, in the case of phosphoserine/threonine-containing peptides, collision-activated dissociation generates, additionally to the usual b- and ypeptide fragments, a very intense fragment corresponding to the neutral loss of phosphoric acid $\left(\mathrm{H}_{3} \mathrm{PO}_{4}, 97.98 \mathrm{Da}\right)$ from the phosphorylated peptide. This approximately $98 \mathrm{Da}$ neutral loss is characteristic of phosphoserine or phosphothreonine (but not phosphotyrosine) and its observation confirms that a phosphopeptide has been fragmented. At times, it is observed not only for the precursor (fragmented peptide) but also for some of the phosphorylated $\mathrm{b}$ - and $\mathrm{y}$-ions. The phosphopeptides reported in Table 1 all bear phosphate 
groups on either serine or threonine residues (never tyrosine). Consequently and as expected, an approiximately 98 Da neutral loss from the precursor was observed on the MS/MS spectrum for each of them (see Figure 3 for a representative phosphopeptide MS/MS spectrum), validating the presence of a phosphorylated peptide. A comprehensive list of all phosphopeptides observed for each kinase is presented in Supplementary Information, along with the phosphopeptides' annotated MS/MS spectra and MS/MS fragmentation tables.

To add confidence to the localization of those PKC $\beta$ sites, we digested phosphorylated PARP-1 with plasmin, which cleaves the protein in three polypeptides of $29 \mathrm{kDa}, 36 \mathrm{kDa}$ (both $\mathrm{N}$-terminal) and $56 \mathrm{kDa}\left(\mathrm{C}\right.$-terminal) ${ }^{65}$. As expected, only the $56 \mathrm{kDa}$ carboxyterminal fragment was observed to be phosphorylated (data not shown), confirming the absence of a phosphorylation site in the amino-terminal portion of the enzyme. Similar results have been obtained by Bauer and collaborators ${ }^{13}$.

The application of MS/MS analysis to in vitro-phosphorylated PARP-1 by PKC $\beta$ resulted in the identification of three phosphoresidues in the vicinity of the protein's C-terminus that were predicted to be PKC phosphorylation sites. All these locations meet a general PKC consensus phosphorylation sequence although serines $\mathrm{S}^{504}$ and $\mathrm{S}^{519}$, which are both localized in the automodification domain, are predicted to be in a more phosphorylatable environment than the threonine $\mathrm{T}^{656}$. This information, coupled with the fact that $\mathrm{S}^{504}$ and $\mathrm{S}^{519}$ are evolutionarily conserved among mammalian PARP-1, as opposed to $\mathrm{T}^{656}$, makes them good candidates for the study of PKC-dependent PARP-1 regulation. 
In vitro phosphorylation of PARP-1 by Erk-1, Erk-2, CKII, CDK5, JNK1 and CamK-II was also examined relative to PKC $\beta$. Fig. $2 \mathrm{~B}$ shows that all these kinases phosphorylate PARP-1, albeit at lower levels than PKC $\beta$. Ten phosphopeptides were identified by LCMS/MS in PARP-1 samples incubated with Erk-1 (Table-1). Kauppinen and collaborators have reported the phosphorylation of PARP-1 by Erk-1 ${ }^{18}$ but were not able to identify the phosphorylation sites using mass spectrometry. Additional mass spectrometry analysis of Erk-2 phosphorylation products led to the detection of a doubly-phosphorylated phosphopeptide $\left({ }^{368}\right.$-TPPPSTASAPAAVNSSAS- ${ }^{385}$, Kauppinen T., personnal communication) bearing three potential phosphorylation sites: $\mathrm{T}^{368}, \mathrm{~S}^{372}$, and $\mathrm{T}^{373}$. Mutation studies revealed that phosphorylation of $\mathrm{S}^{372}$ and $\mathrm{T}^{373}$ activates PARP-1 activity while phosphorylation of $\mathrm{T}^{368}$ has no effect on PARP-1 activity. We also identified a singly phosphorylated Erk-1 phosphopeptide phosphorylated at either $\mathrm{T}^{368}, \mathrm{~S}^{372}, \mathrm{~T}^{373}$, or $\mathrm{S}^{374}$ (Table 1). Precise localization of phosphorylated residues in peptides containing multiple $\mathrm{S} / \mathrm{T} / \mathrm{Y}$ is particularly challenging. To assist us in the interpretation of MS data, we used a probability-based approach that measures the probability of correct phosphorylation site assignment based on the presence and intensity of site-determining ions in the MS/MS spectrum $^{49}$. This approach generates a score, called the Ascore, for each S/T/Y amino acid residues found in the phosphopeptide. It has been shown that greater than $99.8 \%$ of peptides with Ascore $\geq 19$ were localized correctly in the large datasets used to validate the statistical approach $^{49}$. For several of the phosphopeptides observed in this study, the Ascore values indicated that the phosphorylation sites could be confidently assigned but others still lack site-determining MS/MS ions such as the Erk-1 phosphopeptide 
IFPPETSASVAATPPPSTASAPAAVNSSASADKPLSNMK bearing an equivocal phosphorylation site (either $\mathrm{T}^{368}, \mathrm{~S}^{372}, \mathrm{~T}^{373}$, or $\mathrm{S}^{374}$; Table 1). In silico, kinase-specific phosphorylation site prediction can make a contribution to the interpretation of phosphopeptide spectra and help determine which sites should be given further experimental consideration.

Two independent LC-MS/MS investigations to discover CKII phosphosites failed even though CKII does phosphorylate PARP-1 in vitro (Fig. 2B). This is likely due to the low level of phosphorylation obtained after incubation with CKII, and/or to intrinsic characteristics of the CKII-phosphorylated tryptic peptides which prevented their mass spectrometric detection (too large a mass, too many positive charges, low ionizability, low solubility, etc.).

Recently, Ju and collaborators ${ }^{23}$ proposed a model of neurogenic gene activation in which phosphorylation of PARP-1 by CaMK-II is a central event. We therefore tested whether this kinase could phosphorylate PARP-1 in vitro. Fig. 2B shows that CaMK-II can phosphorylate PARP-1 although at a much lower level than the other kinases tested. Even though PARP-1 is barely phosphorylated by CaMK-II, two phosphopeptides were identified for this kinase (Table 1), revealing potential CaMK-II phosphorylation sites ambiguously positioned respectively at $\mathrm{S}^{257} / \mathrm{T}^{258}$ and $\mathrm{S}^{782} / \mathrm{S}^{785} . \mathrm{S}^{257}$ and $\mathrm{S}^{782}$ are, however, the only sites predicted by Phoscan to be within a CaMK-II phosphorylation motif (Table 2). 
Identification of phophorylation sites from PARP-1 phosphorylated in vitro by JNK1 and CDK5 led to the identification of the same phosphoresidues as those obtained with CaMKII (Table 1). As seen on figure 2B, the level of phosphorylation achieved by Erk-2, CKII, CDK5, JNK1 and CaMK-II is significantly lower than that of PKC $\beta$ and Erk-1 for which we identified several unique phosphorylation sites. This suggests that, for Erk-2, CKII, CDK5, JNK1 and CaMK-II, the low stochiometry of phosphorylated PARP-1 relative to the unphosphorylated state prevents optimal mass spectrometric analysis. Indeed, the analysis of phosphorylated peptides by mass spectrometry has always been challenging because of their intrinsically low abundance and poor ionization.

The recombinant PARP-1 used in the present study has been produced in SF-9 insect cells. We were aware that endogenously in vivo phosphorylated sites could be present prior to phosphorylation assays. To better understand these in vivo phosphorylated sites and to distinguish them from kinase-specific phosphorylation sites, we also performed for PARP-1 a general mass spectrometric screening of endogenously phosphorylated residues.

However, because of the low stoichiometry and therefore low abundance of endogenously phosphorylated PARP-1, a phosphopeptide enrichment step was required in order to increase our chance of acquiring phosphopeptide spectra. Several approaches have been developed to enrich phosphoproteins and phosphopeptides for MS analysis ${ }^{66}$ such as antiphosphoresidue immunoprecipitation, affinity chromatography and chemical modification. Immobilized metal affinity chromatography (IMAC) and titanium dioxide $\left(\mathrm{TiO}_{2}\right)$ chromatography are two common methods performed to selectively enrich phosphopeptides based on the chemical affinity of these resins to the phosphate group. 
IMAC and $\mathrm{TiO}_{2}$ are complementary since they tend to exhibit differential affinities towards mono- or multiphosphorylated peptides. Recent optimization of the $\mathrm{TiO}_{2}$ procedure makes it more appropriate for some applications and specifically for matrix-assisted laser desorption ionisation (MALDI) mass spectrometry ${ }^{67}$. We chose to enrich the tryptic digest using $\mathrm{TiO}_{2}$ because we wanted to analyze the enriched tryptic extract using both Electrospray ionization (ESI) and MALDI as these two ionisation modes are known to lead to the detection of complementary sets of peptides and thus increase protein sequence coverage ${ }^{68}$. Three PARP-1 phosphopeptides were identified from MALDI MS analysis of $\mathrm{TiO}_{2}$ enriched peptide extracts (Table 1). Unfortunately, these phosphopeptides were identified solely through their measured mass-to-charge ratio as ion signal intensity was weak and MS/MS signal did not rise above noise level. On the other hand, LC-MS/MS with the LTQ led to the MS/MS confirmation of one the phosphopeptide observed by MALDI, as well as the MS/MS identification of three phosphopeptides not seen by MALDI (Table 1). All in all, six phosphopeptides were detected, confirming the endogenous phosphorylation of PARP-1 produced in insect cells.

Dephosphorylation of recombinant human PARP-1 with calf intestinal alkaline phosphatase (CIAP) has been shown to drastically reduce its enzymatic activity ${ }^{18}$, suggesting that phosphorylated residues are essential to PARP-1 catalytic activity. Table 2 summarizes the information stored in Table 1 by displaying it in a simplified array format. This enables a global view of PARP-1 endogenous and kinase-specific phosphorylation sites along with corresponding in silico phosphorylation predictions: all the phosphorylation sites identified by mass spectrometry are listed relative to their experimental context and the consensus 
kinase recognition sites predicted by in silico computations. In addition, phosphorylation sites reported in the literature are also shown so as to complete the list of currently-known phosphorylation sites for both PARP-1 and PARG. Of particular interest is $\mathrm{S}^{782}$, a phosphosite found by MALDI TOF MS of endogenously phosphorylated PARP-1 and confirmed by LC MS/MS. This site has been consistently identified with high confidence for Erk1, CamK-II, JNK1 and CDK5, which is not unexpected since it was present prior to the phosphorylation assay. Its identification is recurrent in most of the analyzed samples and it is thus likely to be an authentic endogenously phosphorylated residue. In a similar way, the phosphorylation site $\mathrm{S}^{257} / \mathrm{T}^{258}\left(\mathrm{~S}^{257}\right.$ or $\left.\mathrm{T}^{258}\right)$ found within two phosphopeptides from endogenous PARP-1 was also found in Erk-1, CaMK-II and JNK1. The repeated identification of the same phosphorylated residues from independent phosphorylation assays strongly supports its validation as an actual phosphorylation site.

Knowing which sites are phosphorylated endogenously in PARP-1 is not only important for the understanding of PARP-1 regulation under physiological conditions but also to add confidence in the identification of $\mathrm{S} / \mathrm{T} / \mathrm{Y}$ residues phosphorylated by specific kinases. Considering the phosphorylation sites present in recombinant PARP-1 prior to the in vitro assays, we can isolate $\mathrm{T}^{335}$ as a unique endogenous phosphosite not detected in any of the in vitro kinase-specific phosphorylated extracts. Likewise, $\mathrm{S}^{41}, \mathrm{~S}^{177}, \mathrm{~T}^{420 / 422}, \mathrm{~S}^{455}$ and $\mathrm{S}^{542}$ are specific to Erk-1 while $\mathrm{S}^{504}$ and $\mathrm{S}^{519}$ are specific to PKC $\beta$. Alternatively, we could have dephosphorylated recombinant PARP-1 with a phosphatase prior to the in vitro kinases assays in order to overcome the ambiguity in the assignement of a phosphosite to a specific kinase. However, it can be challenging to work with phosphatases when looking for low- 
abundance phosphorylation sites. Moreover, these endogenous phosphorylation sites seem to guide the phosphorylatability of PARP-1 with respect to some kinases as dephosphorylated PARP-1 does not achieve the same level of phosphorylation than untreated PARP-1 and significant impacts PARP-1 activity (Gagné J.-P. et al., unpublished data).

\subsubsection{PARG phosphomapping}

At the time this study was undertaken, the only posttranslational modification known for PARG was an endogenous phosphorylation at $\mathrm{S}^{316}$, which is located next to a caspase cleavage site, identified in a large-scale phosphorylation analysis of HeLa nuclear proteins 47. This position is predicted to be an evolutionarily conserved CKII consensus

phosphorylation site ${ }^{10}$. In recent years, several lines of evidence have indicated that CKII is involved in the regulation of apoptosis, cell cycle and the DNA-damage response ${ }^{69-72}$ (reviewed in ${ }^{73}$ ), three biological processes in which pADPr has been clearly shown to have regulatory functions ${ }^{74}$. The over-representation of putative CKII phosphorylation sites in the $\mathrm{N}$-terminal regulatory domain of PARG, especially in the acidic region that bears the two caspase cleavage sites, also inspired us to determine whether or not CKII could actually phosphorylate PARG. Using affinity-purified PARG over-expressed in E.coli, we found that PARG is indeed a good substrate for CKII (Figure 2C) and validated by mass spectrometry $\mathrm{S}^{316}$ as a CKII phosphorylation site (Table 1). 
We also identified 7 other phosphorylation sites and, out of these, 6 are located in the Nterminal putative regulatory region (Table 1). Furthermore, a study from Villen et al. ${ }^{48}$ identified additional phosphorylation sites $\left(S^{137}, S^{261}, S^{264}\right)$ in the N-terminal region of PARG (Table 2). Therefore, besides $\mathrm{S}^{962 / 966}$ located close to the C-terminus, all identified phosphorylation sites were found in the putative regulatory domain of PARG, raising the possibility that phosphorylation events might modulate PARG functions. For instance, one could hypothesize that the addition of the negative charges from the phosphate groups could impair binding of negatively charged pADPr to PARG and thus modify the affinity of PARG to its substrate. Phosphorylation of PARG could also result in unexpected conformational changes with consequences on activity and protein-protein interactions, or caspase cleavage protection as this has been reported for the caspase substrates $\mathrm{Bid}^{72}$, Max ${ }^{75}$ and PTEN ${ }^{71}$. Further work is in progress to test these hypotheses.

In view of the scarcity of information regarding PARG regulation mechanisms and the lack of known effectors that could be involved in its control, the phosphomapping of PARG phosphorylation sites coupled with mutagenesis studies to determine whether phosphorylation sites are linked to changes in PARG behaviour may significantly improve our understanding of PARG regulation.

4.5 Coupling in silico prediction of phosphorylation sites to phosphopeptide analysis

As it can be seen from Table 2, several phosphopeptides bear ambiguity on the exact phosphorylation site; however computational prediction algorithms such as NetPhosK and 
Phoscan can help determine the exact phosphorylation sites. The determination of a highlyprobable phosphorylation site assigned to a specific kinase is particularly valuable for the design of mutagenesis experiments. The phosphorylation potentials shown in Figure 1A and 1B corresponding to phosphorylation sites within conserved domains of PARP-1 and PARG may also be used to target the most relevant positions related to functional regulation by specific kinases. For example, one could prioritize $\mathrm{S}^{257}$ over $\mathrm{T}^{258}$ to be mutagenized in a functional assay since, in the PARP-1 phosphopeptide KVCpSpTNDLKELLIFNK from which these sites originate, $\mathrm{S}^{257}$ is predicted to be in an environment highly favorable to phosphorylation compared to $\mathrm{T}^{258}$. Predicted kinasespecific phosphorylation sites in phosphopeptides bearing ambiguous phosphosites should also be favoured when planning mutagenesis strategy for functional analysis.

Coupling computational approaches to MS analysis contributes to more targeted mutagenesis experiments which could result in significantly reduced validation procedures. The consolidation of this information with characterized protein domains, motifs or evolutionarily conserved regions will further help us in unravelling the functionality of posttranslational modifications.

4.6 Structure-function modeling of phosphorylated residues of PARP-1 in functional domains

The localization of phosphorylated residues in PARP-1 revealed by mass spectrometry can be coupled to conformational analysis of the crystallographic data. In contrast to PARG, for which there is no three-dimensional structure available, most of the PARP-1 structure has 
been determined. Thus, localization of the phosphorylated residues in three-dimensional models was investigated. Figure 4 shows the localization of phosphoresidues in functional motifs of PARP-1. Phosphorylation sites can be pinpointed in the three zinc-finger motifs (Figure 4a, 4b, 4c), the BRCT domain (Figure 4d), WGR motif (Figure 4e) and the Cterminal catalytic fragment of PARP-1 (Figure 4f). The first N-terminal zinc-finger of PARP-1 is structurally very similar to a homologus domain in DNA ligase III (Figure 4a). A study of the DNA ligase III DNA-binding surface reported by Kulczyk et al. ${ }^{76}$ revealed that amino acids residues located in the extended loop of the finger are strongly affected by the addition of DNA, confirming the importance of the finger loop for DNA-binding, an observation that is very likely to apply to the PARP-1 first zinc-finger. Interestingly, both DNA ligase III and PARP-1 present a highly conserved serine residue in the extended loop required for DNA-binding. This serine residue, $\mathrm{S}^{41}$, has been found in PARP-1 extracts from in vitro phosphorylation assays by Erk-1. Phosphosite $\mathrm{S}^{32}$ was also identified as an endogenous PARP-1 phosphorylation site from MALDI MS analysis (Table-1). This suggest that the DNA-binding of PARP-1 could be regulated by phosphorylating specific residues within the zinc fingers. Following the same idea, figure $4 \mathrm{~b}$ shows two phosphorylation sites in the second zinc-finger of PARP-1 $\left(\mathrm{S}^{177}\right.$ and $\left.\mathrm{S}^{179}\right)$. These sites are located in a highly flexible part of the zinc-finger structure connecting the two helices. In contrast to the first zinc-finger of PARP-1, these phosphorylation sites are not located in the extended loop or the $\beta$-sheets that make contact with DNA so the impact on DNA-binding would probably less dramatic as it was proposed for DNA ligase III. 
Figure $4 \mathrm{c}$ represents the third zinc-finger of PARP-1 in a dimer model developed by Langelier et al. ${ }^{77}$. Indeed, apart from being a zinc-binding structure that also mediates interdomain communication, the authors reported that this motif self-associates in the crystal lattice, forming an extensive homodimer interface. Endogenous phosphorylations sites $\left(\mathrm{S}^{257} / \mathrm{T}^{258}\right.$ and $\mathrm{T}^{335}$ ) were localized near the dimer interface (Figure $4 \mathrm{c}$ ) thus inviting to the speculation that phosphorylation of this position might affect the protein dimerization interface.

Phosphosites unique to Erk-1 were localized in the BRCT domain of PARP-1 (Figure 4d), an important protein-protein interaction motif found predominantly in proteins involved in cell cycle checkpoint functions responsive to DNA damage (e.g. XRCC1, DNA ligase III, BRCA1, 53BP1, MDC1). The functional consequences of phosphorylating the BRCT domain of PARP-1 are unknown. However, the kinase Aurora-B has been reported to bind PARP-1 via the BRCT motif and to phosphorylate PARP-1 ${ }^{78}$. The BRCT domain of PARP-1 is highly similar to the BRCT domain of XRCC1 (Figure 4d). The core of the BRCT domain is composed of $\beta$-sheets important for adequate folding of the structure. Indeed, mutations in the core of XRCC1 BRCT motif $\left(\mathrm{V}^{584}\right.$ and $\left.\mathrm{I}^{585}\right)$ has been shown to disrupt the interaction between XRCC1 and DNA ligase III ${ }^{79} \cdot \mathrm{T}^{420}$ and $\mathrm{S}^{455}$ phosphosites are also localized in the $\beta$-sheets of PARP-1 BRCT domain. It would be interesting to know how a phosphorylated BRCT domain of PARP-1 will behave in a context of proteinprotein interactions with its partners. 
Finally, three phosphosites are found in the PARP regulatory domain within the C-terminal catalytic domain of PARP-1 $\left(\mathrm{Y}^{775}, \mathrm{~S}^{782}, \mathrm{~S}^{785}\right.$ or $\left.\mathrm{S}^{786}\right)$ that could also be involved in the regulation of PARP-1 activity. These sites are localized in a flexible and highly accessible loop of the catalytic domain (Figure 4f). Previous studies have shown that phosphorylation sites are mainly located in parts of proteins without regular structure such as hinges and loops ${ }^{80-83}$. A closer look at the N-terminal domain of PARG reveals that this region is predicted to be an extended loop region. Indeed, a secondary structure prediction of PARG

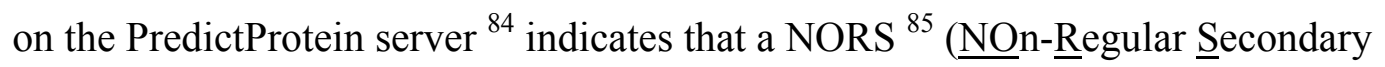
Structure) spans the entire putative regulatory domain of PARG where most of the phosphorylation sites are clustered.

Following the same idea, unstructured interdomain regions of PARP-1 are thought to present structural attributes favorable to phosphorylation. This seems to be applicable to this study since several phosphorylation sites are localized within inter domain regions of PARP-1 (Figure 1a) for which no structural data is available. However, these phsophorylation sites located ouside characterized motif have proven to be of great importance in the regulation of PARP-1 activity. Indeed, the phosphosites $\mathrm{S}^{372}$ and $\mathrm{T}^{373}$ located between the third zinc finger and the BRCT domain of PARP-1 (Figure 1a) are Erk phosphorylation sites reported by Kaupinnen et al. to be required for full activation of PARP- ${ }^{18}$. We also identified these sites in an endogenously phosphorylated peptide bearing multiple phosphorylatable residues (IFPPETSASVAApTPPPpSpTApSAPAAVNSSASADKPLSNMK, see Table-1). A cluster of phsophorylated serine residues is also present between the BRCT and the WGR domain of PARP-1 $\left(\mathrm{S}^{509}, \mathrm{~S}^{519}, \mathrm{~S}^{542}\right.$, Figure 
1a) and a single phosphothreonine residue $\left(\mathrm{T}^{656}\right)$ is located between the WGR and the PRD domains. The analysis of the 3D coordinates of the WGR motif of PARP-1 that extend outside the motif in the N-terminal direction shows that phosphoresidues $\mathrm{S}^{519}$ and $\mathrm{S}^{542}$ are actually in unstructured and flexible regions (Figure 4e). Inspection of the tertiary structure of PARP-1 coupled to localization of the phosphorylations sites revealed by mass spectrometry analysis is particularly interesting in a structure-function evaluation and to target phsophosites for further experimental validation.

4.7 In vivo validation of some PARP-1 phosphosites in a context of DNA damage signaling

The recruitment of PARP-1 at sites of DNA damage and its subsequent activation result in a rapid and transient accumulation of pADPr. Recent studies suggest a mechanism by which pADPr-binding interaction may contribute to the initial damage signalling that leads to the recruitment of DNA repair proteins ${ }^{56,86,87}$. Because altering the properties of PARP1 recruitment during the initial DNA-damage response is likely to have a large impact on the damage signalling and the subsequent DNA repair process, we analyzed the recruitment kinetics of selected phosphorylation sites in this context.

The analysis of site-specific PARP-1 phosphorylation in response to direct phosphorylation by Erk1 and Erk2, reported by Kauppinen et al. ${ }^{18}$, is the only study that provides a link between PARP-1 phosphorylation at specific sites and enzymatic functions. In our study, we report several new Erk1 phosphorylation sites (Table 1). Of particular interest is the 
Erk-1 phosphosite found at $\mathrm{S}^{41}$, a typical (S/T)P proline-directed MAPK phosphorylation consensus found in a variety of substrates of MAP-related kinases. The phosphosite $\mathrm{S}^{41}$ is similar to the $\mathrm{S}^{372}$ phosphosite validated by Kauppinen and colleagues, which was another consensus proline-directed MAPK phosphorylation site. Moreover, the evolutionarily conserved $\mathrm{S}^{41}$ is predicted by both kinase-specific computational tools as a MAPK phosphorylation site (Table 1). Therefore, $\mathrm{S}^{41}$ and other proximal phosphorylation sites ( $\mathrm{S}^{27}$ and $\mathrm{S}^{32}$ ) were selected as good candidates for in vivo validation.

Using laser microirradiation-induced DNA damage, we analyzed the recruitment kinetics of GFP-PARP-1 phosphomutants within the first N-terminal zinc finger domain of PARP-1. Single and triple glutamate- and aspartate-substituted derivatives designated S27D-S32ES41E were generated to mimic a permanently phosphorylated protein. Figure 5 shows that a single mutation at $\mathrm{S}^{41}$ severely impairs the recruitment and persistence of PARP-1 at DNA damage sites while similar substitutions at phosphosites $\mathrm{S}^{27}$ and $\mathrm{S}^{32}$ show little divergence compared to the wild-type protein. A triple substitution including proximal serine phosphorylation sites (S27D-S32E-S41E) do not show additive effects indicating that the altered kinetics observed for the triple phosphomutant are a consequence of the single contribution of site $\mathrm{S}^{41}$. This observation suggests that the phosphorylation of PARP-1 may be part of a regulation mechanism to delocalize the enzyme from the DNA damage site and limit the extent of poly(ADP-ribosylation) and NAD depletion in the nucleus. This is the first demonstration of phosphorylation-dependent dynamics of PARP-1 using live cell imaging combined with laser microirradiation. 


\section{Concluding remarks}

Cataloguing PARP-1 and PARG protein phosphorylation states represents a major challenge given that this posttranslational modification occurs at low stoichiometry, includes dynamic and reversible states with spatiotemporal regulation within cells. Even though global phosphoproteome analysis has provided valuable information and has led to the discovery of new phosphorylation sites, these are primarily abundant sites. Indeed, almost all phosphopeptides found with large-scale analysis or with endogenously-modified recombinant proteins correspond to high-potential phosphorylation sites. More targeted strategies, such as that used in the present study, which include specific coverage of kinases and phosphopeptide enrichment techniques will deepen of understanding of how the various phosphorylated isoforms of PARP-1 and PARG regulate the functions and activities of these enzymes

\section{Acknowledgements}

We would like to thank the Taplin Biological Mass Spectrometry Facility, Department of Cell Biology (Harvard Medical School, Boston, U.S.A.) for processing some PARP-1 and PARG samples for the determination of phosphorylation sites. This work was supported by grants from Genome Canada, Génome Québec, Genome Prairie and Genome Alberta. JPG is supported by a doctoral training award from Fonds de la Recherche en Santé du Québec (FRSQ). AD is funded by a Canadian Institutes of Health Research (CIHR) training grant 
in functional genomics. MJH is a senior scholar of the Alberta Heritage Foundation for Medical Research. GGP holds a Canada Research Chair in Proteomics.

\section{Supporting Information Available}

Table of generic and kinase-specific predictions of phosphorylation for PARP-1 and PARG and MS/MS data of phosphopeptides. This material is available free of charge via the Internet at http://pubs.acs.org. 


\section{Figure legends}

Fig. 1. Diagrammatic representation of PARP-1 (A) and PARG (B) phosphorylation sites. An in silico-based prediction approach was used for the analysis of phosphorylation sites within the primary amino acid sequences of PARP-1 and PARG. The phosphorylation sites were predicted by the NetPhos program which evaluates serine, threonine and tyrosine $(\mathrm{S} / \mathrm{T} / \mathrm{Y})$ phosphorylation sites in eukaryotic proteins by applying a neural network method that recognizes the amino acid patterns around the phosphorylated residue. Each colourcoded diagram depicts predicted $\mathrm{S} / \mathrm{T} / \mathrm{Y}$ phosphorylation sites according to NetPhos algorithm in relation with the protein amino acid sequence. Schematic organization of functional domains is shown with experimentally-determined phosphorylation sites (see Table 2 for a description of the experimental context). Dashed boxes representing highprobability areas of phosphorylation are depicted with respect to functional domains. PARG's isoelectric points are estimated for the N-terminal putative regulatory domain, the acidic region and the catalytic C-terminal domain. N: amino-terminal, $\mathrm{C}$ : carboxy-terminal, ZF: zinc fingers, NLS: nuclear localization signal, NES: nuclear export signal, MLS: mitochondrial localization signal, AMD: automodification domain, LZM : putative leucine zipper-like motif, PADR: Poly(ADP-ribose) polymerases-associated domain, BRCT : BRCA1 carboxy-terminal domain, PS: PARP signature, PRD: PARP regulatory domain, WGR: tryptophan(W)-glycine(G)-arginine(R) domain. 
Fig. 2. In vitro phosphorylation of recombinant PARP-1 and PARG. Kinase assays consisted of incubating purified PARP-1 and PARG with the protein kinase in the presence of $\left[\gamma^{32}{ }^{32} \mathrm{P}\right] \mathrm{ATP}$ as described in Materials and Methods. The reaction products were separated by SDS-PAGE, dried and autoradiographied. (A) PARP-1 is phosphorylated by PKC $\alpha$ and $\beta$. (B) PARP-1 is phosphorylated by various kinases suspected to be involved in PARP-1-dependent pathways. (C) PARG is a substrate of CKII. In vitro phosphorylation of PARG by CKII was assayed in presence of $\left[\gamma^{32} \mathrm{P}\right] \mathrm{ATP}$. The corresponding coomassie bluestained PARG protein band is shown under each lane.

Fig 3. Tandem mass spectrum of a representative PARP-1 tryptic phosphopeptide. Purified recombinant PARP-1 expressed in SF-9 cells was digested with trypsin and the resulting peptide mixture was enriched in phosphopeptides using titanium dioxide (TiO2). Analysis was performed on a LTQ linear ion trap mass spectrometer. MS/MS spectra were assigned with Sequest using a two-pass approach as described in Materials and Methods. Scaffold was used to filter MS/MS-based identifications and annotate MS/MS spectra. The spectrum shows the phosphopeptide GGpSDDSSKDPIDVNYEK which contains a phosphoserine residue $\left(\mathrm{S}^{782}\right)$ identified by the analysis of the peptide's $y$ and $b$ fragments. An MS/MS peptide fragmentation table of $y$ and $b$ ions is shown under the spectrum. The prominent neutral loss of a phosphate group (-98 Da) from the phosphopeptide precursor is indicated by a green arrow. Sequest: $\mathrm{XCorr}=4.85$, deltaCn $=0.11$; Ascore $=53.3$. See Supplementary Information for zoomed regions of the spectrum. 
Fig 4. Structural models of PARP-1 domains. Schematic representations of PARP-1 domains that contain phosphoresidues are illustrated. Alpha helices (cyan and green) and $\beta$ sheets are rendered as solid ribbons. Phosphorylation sites identified by MS analysis are indicated by an arrow. (A) The first zinc-finger domain of PARP-1 (Protein Data Bank (PDB) accession code 2DMJ) is depicted with the homologous zinc-finger of DNA ligase III (1UW0). Side chains of amino acid residues that were shown to be required for DNAbinding in the corresponding extended loop of DNA ligase III zinc finger are labelled in green. Structure was modelled after that proposed by Kulczyk et al. ${ }^{76}$. The position of the zinc atom is depicted by a black sphere. (B) The second zinc-finger of PARP-1 (2CS2) is modelled in the same orientation as the first zinc-finger. (C) Schematic representation of PARP-1 dimer interface in the third zinc-finger (2RIQ) as proposed by Langelier et al. ${ }^{77}$. (D) Homologuous BRCT domains of PARP-1 (2COK) and XRCC1 (1CDZ) are modelled according to Zhang et al. ${ }^{88}$. Point mutations (V584D and I585D) in the BRCT domain of $\mathrm{XRCC} 1$ that disrupt protein-protein interactions are shown in green. (E) The WGR domain (2CR9) of PARP-1. (F) The C-terminal catalytic domain of PARP-1 (1WOK) complexed with an inhibitor (black sticks).

Fig 5. $\mathrm{S}^{41}$ Erk-1 phosphorylation site located in the first zinc finger motif of PARP-1 reveals altered recruitment kinetics at sites of DNA damage following laser microirradiation. The spatial dynamics of GFP-tagged single and triple glutamate- and aspartate-substituted derivatives that mimic a permanently phosphorylated protein (S27DS32E-S41E) were investigated by micro irradiation-induced DNA damage using a 750-nm titanium-sapphire laser. The relative fluorescence intensity at the microirradiated region of 
HeLa cells was normalized and plotted as a function of time after integrating data from at least five low-expressing cells. The error bars represent the standard error. 


\section{References}

1. Meyer-Ficca, M. L.; Meyer, R. G.; Jacobson, E. L.; Jacobson, M. K., Poly(ADPribose) polymerases: managing genome stability. Int J Biochem Cell Biol 2005, 37, (5), $920-6$.

2. Malanga, M.; Althaus, F. R., The role of poly(ADP-ribose) in the DNA damage signaling network. Biochem Cell Biol 2005, 83, (3), 354-64.

3. Faraone-Mennella, M. R., Chromatin architecture and functions: the role(s) of poly(ADP-RIBOSE) polymerase and poly(ADPribosyl)ation of nuclear proteins. Biochem Cell Biol 2005, 83, (3), 396-404.

4. Petermann, E.; Keil, C.; Oei, S. L., Importance of poly(ADP-ribose) polymerases in the regulation of DNA-dependent processes. Cell Mol Life Sci 2005, 62, (7-8), 731-8.

5. Bouchard, V. J.; Rouleau, M.; Poirier, G. G., PARP-1, a determinant of cell survival in response to DNA damage. Exp Hematol 2003, 31, (6), 446-54.

6. Koh, D. W.; Dawson, T. M.; Dawson, V. L., Mediation of cell death by poly(ADPribose) polymerase-1. Pharmacol Res 2005, 52, (1), 5-14.

7. Andrabi, S. A.; Kim, N. S.; Yu, S. W.; Wang, H.; Koh, D. W.; Sasaki, M.; Klaus, J. A.; Otsuka, T.; Zhang, Z.; Koehler, R. C.; Hurn, P. D.; Poirier, G. G.; Dawson, V. L.; Dawson, T. M., Poly(ADP-ribose) (PAR) polymer is a death signal. Proc Natl Acad Sci U S $A$ 2006, 103, (48), 18308-13. 
8. Yu, S. W.; Andrabi, S. A.; Wang, H.; Kim, N. S.; Poirier, G. G.; Dawson, T. M.; Dawson, V. L., Apoptosis-inducing factor mediates poly(ADP-ribose) (PAR) polymerinduced cell death. Proc Natl Acad Sci U S A 2006, 103, (48), 18314-9.

9. Schreiber, V.; Dantzer, F.; Ame, J. C.; de Murcia, G., Poly(ADP-ribose): novel functions for an old molecule. Nat Rev Mol Cell Biol 2006, 7, (7), 517-28.

10. Gagne, J. P.; Hendzel, M. J.; Droit, A.; Poirier, G. G., The expanding role of poly(ADP-ribose) metabolism: current challenges and new perspectives. Curr Opin Cell Biol 2006, 18, (2), 145-51.

11. Burkle, A., Poly(ADP-ribose). The most elaborate metabolite of NAD+. Febs $J$ 2005, 272, (18), 4576-89.

12. Tanaka, Y.; Koide, S. S.; Yoshihara, K.; Kamiya, T., Poly (ADP-ribose) synthetase is phosphorylated by protein kinase C in vitro. Biochem Biophys Res Commun 1987, 148, (2), 709-17.

13. Bauer, P. I.; Farkas, G.; Buday, L.; Mikala, G.; Meszaros, G.; Kun, E.; Farago, A., Inhibition of DNA binding by the phosphorylation of poly ADP-ribose polymerase protein catalysed by protein kinase C. Biochem Biophys Res Commun 1992, 187, (2), 730-6.

14. Bauer, P. I.; Farkas, G.; Mihalik, R.; Kopper, L.; Kun, E.; Farago, A., Phosphorylation of poly(ADP-ribose)polymerase protein in human peripheral lymphocytes stimulated with phytohemagglutinin. Biochim Biophys Acta 1994, 1223, (2), 234-9. 
15. Aoufouchi, S.; Shall, S., Regulation by phosphorylation of Xenopus laevis poly(ADP-ribose) polymerase enzyme activity during oocyte maturation. Biochem $J$ 1997, 325 ( Pt 2), 543-51.

16. Beckert, S.; Farrahi, F.; Perveen Ghani, Q.; Aslam, R.; Scheuenstuhl, H.; Coerper, S.; Konigsrainer, A.; Hunt, T. K.; Hussain, M. Z., IGF-I-induced VEGF expression in HUVEC involves phosphorylation and inhibition of poly(ADP-ribose)polymerase. Biochem Biophys Res Commun 2006, 341, (1), 67-72.

17. LeRoith, D.; Roberts, C. T., Jr., The insulin-like growth factor system and cancer. Cancer Lett 2003, 195, (2), 127-37.

18. Kauppinen, T. M.; Chan, W. Y.; Suh, S. W.; Wiggins, A. K.; Huang, E. J.;

Swanson, R. A., Direct phosphorylation and regulation of poly(ADP-ribose) polymerase-1 by extracellular signal-regulated kinases 1/2. Proc Natl Acad Sci U S A 2006, 103, (18), $7136-41$.

19. Cohen-Armon, M.; Visochek, L.; Rozensal, D.; Kalal, A.; Geistrikh, I.; Klein, R.; Bendetz-Nezer, S.; Yao, Z.; Seger, R., DNA-independent PARP-1 activation by phosphorylated ERK2 increases Elk1 activity: a link to histone acetylation. Mol Cell 2007, 25, (2), 297-308.

20. Ethier, C.; Labelle, Y.; Poirier, G. G., PARP-1-induced cell death through inhibition of the MEK/ERK pathway in MNNG-treated HeLa cells. Apoptosis 2007, 12, (11), 203749. 
21. Zhang, S.; Lin, Y.; Kim, Y. S.; Hande, M. P.; Liu, Z. G.; Shen, H. M., c-Jun Nterminal kinase mediates hydrogen peroxide-induced cell death via sustained poly(ADPribose) polymerase-1 activation. Cell Death Differ 2007, 14, (5), 1001-10.

22. Rosenthal, D. S.; Simbulan-Rosenthal, C. M.; Iyer, S.; Smith, W. J.; Ray, R.; Smulson, M. E., Calmodulin, poly(ADP-ribose)polymerase and p53 are targets for modulating the effects of sulfur mustard. J Appl Toxicol 2000, 20 Suppl 1, S43-9.

23. Ju, B. G.; Solum, D.; Song, E. J.; Lee, K. J.; Rose, D. W.; Glass, C. K.; Rosenfeld, M. G., Activating the PARP-1 sensor component of the groucho/ TLE1 corepressor complex mediates a CaMKinase IIdelta-dependent neurogenic gene activation pathway. Cell 2004, 119, (6), 815-29.

24. Midorikawa, R.; Takei, Y.; Hirokawa, N., KIF4 motor regulates activity-dependent neuronal survival by suppressing PARP-1 enzymatic activity. Cell 2006, 125, (2), 371-83.

25. Maruyama, T.; Nara, K.; Yoshikawa, H.; Suzuki, N., Txk, a member of the nonreceptor tyrosine kinase of the Tec family, forms a complex with poly(ADP-ribose) polymerase 1 and elongation factor 1alpha and regulates interferon-gamma gene transcription in Th1 cells. Clin Exp Immunol 2007, 147, (1), 164-75.

26. Blaydes, J. P.; Hupp, T. R., DNA damage triggers DRB-resistant phosphorylation of human p53 at the CK2 site. Oncogene 1998, 17, (8), 1045-52. 
27. McKendrick, L.; Milne, D.; Meek, D., Protein kinase CK2-dependent regulation of p53 function: evidence that the phosphorylation status of the serine 386 (CK2) site of p53 is constitutive and stable. Mol Cell Biochem 1999, 191, (1-2), 187-99.

28. Keller, D. M.; Zeng, X.; Wang, Y.; Zhang, Q. H.; Kapoor, M.; Shu, H.; Goodman, R.; Lozano, G.; Zhao, Y.; Lu, H., A DNA damage-induced p53 serine 392 kinase complex contains CK2, hSpt16, and SSRP1. Mol Cell 2001, 7, (2), 283-92.

29. O'Brien, K. A.; Lemke, S. J.; Cocke, K. S.; Rao, R. N.; Beckmann, R. P., Casein kinase 2 binds to and phosphorylates BRCA1. Biochem Biophys Res Commun 1999, 260, (3), 658-64.

30. Loizou, J. I.; El-Khamisy, S. F.; Zlatanou, A.; Moore, D. J.; Chan, D. W.; Qin, J.; Sarno, S.; Meggio, F.; Pinna, L. A.; Caldecott, K. W., The protein kinase CK2 facilitates repair of chromosomal DNA single-strand breaks. Cell 2004, 117, (1), 17-28.

31. Morales, J. C.; Carpenter, P. B., Breaking in a new function for casein kinase 2. Sci Aging Knowledge Environ 2004, 2004, (22), pe24.

32. Schultz, M. C., DNA damage regulation of the RNA components of the translational apparatus: new biology and mechanisms. IUBMB Life 2003, 55, (4-5), 243-7.

33. Teitz, T.; Eli, D.; Penner, M.; Bakhanashvili, M.; Naiman, T.; Timme, T. L.; Wood, C. M.; Moses, R. E.; Canaani, D., Expression of the cDNA for the beta subunit of human casein kinase II confers partial UV resistance on xeroderma pigmentosum cells. Mutat Res 1990, 236, (1), 85-97. 
34. Toczyski, D. P.; Galgoczy, D. J.; Hartwell, L. H., CDC5 and CKII control adaptation to the yeast DNA damage checkpoint. Cell 1997, 90, (6), 1097-106.

35. Kapoor, M.; Lozano, G., Functional activation of p53 via phosphorylation following DNA damage by UV but not gamma radiation. Proc Natl Acad Sci U S A 1998, 95, (6), 2834-7.

36. Keller, D. M.; Lu, H., p53 serine 392 phosphorylation increases after UV through induction of the assembly of the CK2.hSPT16.SSRP1 complex. J Biol Chem 2002, 277, (51), 50206-13.

37. Ghavidel, A.; Schultz, M. C., TATA binding protein-associated CK2 transduces DNA damage signals to the RNA polymerase III transcriptional machinery. Cell 2001, 106, (5), 575-84.

38. Iles, N.; Rulten, S.; El-Khamisy, S. F.; Caldecott, K. W., APLF (C2orf13) is a novel human protein involved in the cellular response to chromosomal DNA strand breaks. Mol Cell Biol 2007, 27, (10), 3793-803.

39. Bekker-Jensen, S.; Fugger, K.; Danielsen, J. R.; Gromova, I.; Sehested, M.; Celis, J.; Bartek, J.; Lukas, J.; Mailand, N., Human Xip1 (C2orf13) is a novel regulator of cellular responses to DNA strand breaks. J Biol Chem 2007, 282, (27), 19638-43.

40. Altaf, M.; Saksouk, N.; Cote, J., Histone modifications in response to DNA damage. Mutat Res 2007, 618, (1-2), 81-90. 
41. Turner, N. C.; Lord, C. J.; Iorns, E.; Brough, R.; Swift, S.; Elliott, R.; Rayter, S.; Tutt, A. N.; Ashworth, A., A synthetic lethal siRNA screen identifying genes mediating sensitivity to a PARP inhibitor. Embo J 2008, 27, (9), 1368-77.

42. Lee, J. H.; Kim, H. S.; Lee, S. J.; Kim, K. T., Stabilization and activation of p53 induced by Cdk5 contributes to neuronal cell death. J Cell Sci 2007, 120, (Pt 13), 2259-71.

43. Lee, J. M.; Dedhar, S.; Kalluri, R.; Thompson, E. W., The epithelial-mesenchymal transition: new insights in signaling, development, and disease. J Cell Biol 2006, 172, (7), 973-81.

44. Ullrich, O.; Diestel, A.; Eyupoglu, I. Y.; Nitsch, R., Regulation of microglial expression of integrins by poly(ADP-ribose) polymerase-1. Nat Cell Biol 2001, 3, (12), $1035-42$.

45. McPhee, T. R.; McDonald, P. C.; Oloumi, A.; Dedhar, S., Integrin-linked kinase regulates E-cadherin expression through PARP-1. Dev Dyn 2008.

46. Walker, J. W.; Jijon, H. B.; Madsen, K. L., AMP-activated protein kinase is a positive regulator of poly(ADP-ribose) polymerase. Biochem Biophys Res Commun 2006, $342,(1), 336-41$.

47. Beausoleil, S. A.; Jedrychowski, M.; Schwartz, D.; Elias, J. E.; Villen, J.; Li, J.; Cohn, M. A.; Cantley, L. C.; Gygi, S. P., Large-scale characterization of HeLa cell nuclear phosphoproteins. Proc Natl Acad Sci US A 2004, 101, (33), 12130-5. 
48. Villen, J.; Beausoleil, S. A.; Gerber, S. A.; Gygi, S. P., Large-scale phosphorylation analysis of mouse liver. Proc Natl Acad Sci U S A 2007, 104, (5), 1488-93.

49. Beausoleil, S. A.; Villen, J.; Gerber, S. A.; Rush, J.; Gygi, S. P., A probability-based approach for high-throughput protein phosphorylation analysis and site localization. Nat Biotechnol 2006, 24, (10), 1285-92.

50. Imami, K.; Sugiyama, N.; Kyono, Y.; Tomita, M.; Ishihama, Y., Automated phosphoproteome analysis for cultured cancer cells by two-dimensional nanoLC-MS using a calcined titania/C18 biphasic column. Anal Sci 2008, 24, (1), 161-6.

51. Blom, N.; Gammeltoft, S.; Brunak, S., Sequence and structure-based prediction of eukaryotic protein phosphorylation sites. J Mol Biol 1999, 294, (5), 1351-62.

52. Blom, N.; Sicheritz-Ponten, T.; Gupta, R.; Gammeltoft, S.; Brunak, S., Prediction of post-translational glycosylation and phosphorylation of proteins from the amino acid sequence. Proteomics 2004, 4, (6), 1633-49.

53. Li, T.; Li, F.; Zhang, X., Prediction of kinase-specific phosphorylation sites with sequence features by a log-odds ratio approach. Proteins 2007.

54. Craig, R.; Beavis, R. C., A method for reducing the time required to match protein sequences with tandem mass spectra. Rapid Commun Mass Spectrom 2003, 17, (20), 23106. 
55. Kersey, P. J.; Duarte, J.; Williams, A.; Karavidopoulou, Y.; Birney, E.; Apweiler, R., The International Protein Index: an integrated database for proteomics experiments. Proteomics 2004, 4, (7), 1985-8.

56. Haince, J. F.; McDonald, D.; Rodrigue, A.; Dery, U.; Masson, J. Y.; Hendzel, M. J.; Poirier, G. G., PARP1-dependent kinetics of recruitment of MRE11 and NBS1 proteins to multiple DNA damage sites. J Biol Chem 2008, 283, (2), 1197-208.

57. Haince, J. F.; Ouellet, M. E.; McDonald, D.; Hendzel, M. J.; Poirier, G. G., Dynamic relocation of poly(ADP-ribose) glycohydrolase isoforms during radiation-induced DNA damage. Biochim Biophys Acta 2006, 1763, (2), 226-37.

58. Ame, J. C.; Jacobson, E. L.; Jacobson, M. K., Molecular heterogeneity and regulation of poly(ADP-ribose) glycohydrolase. Mol Cell Biochem 1999, 193, (1-2), 75-81.

59. Kayukawa, K.; Makino, Y.; Yogosawa, S.; Tamura, T., A serine residue in the Nterminal acidic region of rat RPB6, one of the common subunits of RNA polymerases, is exclusively phosphorylated by casein kinase II in vitro. Gene 1999, 234, (1), 139-47.

60. Allende-Vega, N.; Dias, S.; Milne, D.; Meek, D., Phosphorylation of the acidic domain of Mdm2 by protein kinase CK2. Mol Cell Biochem 2005, 274, (1-2), 85-90.

61. Thomsen, M. S.; Franssen, L.; Launholt, D.; Fojan, P.; Grasser, K. D., Interactions of the basic $\mathrm{N}$-terminal and the acidic $\mathrm{C}$-terminal domains of the maize chromosomal HMGB1 protein. Biochemistry 2004, 43, (25), 8029-37. 
62. Krohn, N. M.; Stemmer, C.; Fojan, P.; Grimm, R.; Grasser, K. D., Protein kinase CK2 phosphorylates the high mobility group domain protein SSRP1, inducing the recognition of UV-damaged DNA. J Biol Chem 2003, 278, (15), 12710-5.

63. Phelps, C. B.; Sengchanthalangsy, L. L.; Huxford, T.; Ghosh, G., Mechanism of I kappa B alpha binding to NF-kappa B dimers. J Biol Chem 2000, 275, (38), 29840-6.

64. Bauer, P. I.; Buki, K. G.; Kun, E., Selective augmentation of histone H1 phosphorylation sites by interaction of poly(ADP-ribose) polymerase and cdc2-kinase: comparison with protein kinase C. Int J Mol Med 2001, 8, (6), 691-3.

65. Buki, K. G.; Kun, E., Polypeptide domains of ADP-ribosyltransferase obtained by digestion with plasmin. Biochemistry 1988, 27, (16), 5990-5.

66. Reinders, J.; Sickmann, A., State-of-the-art in phosphoproteomics. Proteomics 2005, 5, (16), 4052-61.

67. Larsen, M. R.; Thingholm, T. E.; Jensen, O. N.; Roepstorff, P.; Jorgensen, T. J., Highly selective enrichment of phosphorylated peptides from peptide mixtures using titanium dioxide microcolumns. Mol Cell Proteomics 2005, 4, (7), 873-86.

68. Bodnar, W. M.; Blackburn, R. K.; Krise, J. M.; Moseley, M. A., Exploiting the complementary nature of LC/MALDI/MS/MS and LC/ESI/MS/MS for increased proteome coverage. J Am Soc Mass Spectrom 2003, 14, (9), 971-9. 
69. Yamane, K.; Kinsella, T. J., Casein kinase 2 regulates both apoptosis and the cell cycle following DNA damage induced by 6-thioguanine. Clin Cancer Res 2005, 11, (6), $2355-63$.

70. Yamane, K.; Kinsella, T. J., CK2 inhibits apoptosis and changes its cellular localization following ionizing radiation. Cancer Res 2005, 65, (10), 4362-7.

71. Torres, J.; Rodriguez, J.; Myers, M. P.; Valiente, M.; Graves, J. D.; Tonks, N. K.; Pulido, R., Phosphorylation-regulated cleavage of the tumor suppressor PTEN by caspase3: implications for the control of protein stability and PTEN-protein interactions. $J$ Biol Chem 2003, 278, (33), 30652-60.

72. Desagher, S.; Osen-Sand, A.; Montessuit, S.; Magnenat, E.; Vilbois, F.; Hochmann, A.; Journot, L.; Antonsson, B.; Martinou, J. C., Phosphorylation of bid by casein kinases I and II regulates its cleavage by caspase 8. Mol Cell 2001, 8, (3), 601-11.

73. Litchfield, D. W., Protein kinase CK2: structure, regulation and role in cellular decisions of life and death. Biochem J 2003, 369, (Pt 1), 1-15.

74. Hassa, P. O.; Haenni, S. S.; Elser, M.; Hottiger, M. O., Nuclear ADP-ribosylation reactions in mammalian cells: where are we today and where are we going? Microbiol Mol Biol Rev 2006, 70, (3), 789-829.

75. Krippner-Heidenreich, A.; Talanian, R. V.; Sekul, R.; Kraft, R.; Thole, H.; Ottleben, H.; Luscher, B., Targeting of the transcription factor Max during apoptosis: 
phosphorylation-regulated cleavage by caspase- 5 at an unusual glutamic acid residue in position P1. Biochem J 2001, 358, (Pt 3), 705-15.

76. Kulczyk, A. W.; Yang, J. C.; Neuhaus, D., Solution structure and DNA binding of the zinc-finger domain from DNA ligase IIIalpha. J Mol Biol 2004, 341, (3), 723-38.

77. Langelier, M. F.; Servent, K. M.; Rogers, E. E.; Pascal, J. M., A third zinc-binding domain of human poly(ADP-ribose) polymerase-1 coordinates DNA-dependent enzyme activation. J Biol Chem 2008, 283, (7), 4105-14.

78. Monaco, L.; Kolthur-Seetharam, U.; Loury, R.; Murcia, J. M.; de Murcia, G.; Sassone-Corsi, P., Inhibition of Aurora-B kinase activity by poly(ADP-ribosyl)ation in response to DNA damage. Proc Natl Acad Sci US A 2005, 102, (40), 14244-8.

79. Taylor, R. M.; Wickstead, B.; Cronin, S.; Caldecott, K. W., Role of a BRCT domain in the interaction of DNA ligase III-alpha with the DNA repair protein XRCC1. Curr Biol 1998, 8, (15), 877-80.

80. Dunker, A. K.; Brown, C. J.; Lawson, J. D.; Iakoucheva, L. M.; Obradovic, Z., Intrinsic disorder and protein function. Biochemistry 2002, 41, (21), 6573-82.

81. Iakoucheva, L. M.; Radivojac, P.; Brown, C. J.; O'Connor, T. R.; Sikes, J. G.; Obradovic, Z.; Dunker, A. K., The importance of intrinsic disorder for protein phosphorylation. Nucleic Acids Res 2004, 32, (3), 1037-49. 
82. Gnad, F.; Ren, S.; Cox, J.; Olsen, J. V.; Macek, B.; Oroshi, M.; Mann, M., PHOSIDA (phosphorylation site database): management, structural and evolutionary investigation, and prediction of phosphosites. Genome Biol 2007, 8, (11), R250.

83. Collins, M. O.; Yu, L.; Campuzano, I.; Grant, S. G.; Choudhary, J. S., Phosphoproteomic analysis of the mouse brain cytosol reveals a predominance of protein phosphorylation in regions of intrinsic sequence disorder. Mol Cell Proteomics 2008, 7, (7), $1331-48$.

84. Rost, B.; Yachdav, G.; Liu, J., The PredictProtein server. Nucleic Acids Res 2004, 32, (Web Server issue), W321-6.

85. Liu, J.; Rost, B., NORSp: Predictions of long regions without regular secondary structure. Nucleic Acids Res 2003, 31, (13), 3833-5.

86. Haince, J. F.; Kozlov, S.; Dawson, V. L.; Dawson, T. M.; Hendzel, M. J.; Lavin, M. F.; Poirier, G. G., Ataxia telangiectasia mutated (ATM) signaling network is modulated by a novel poly(ADP-ribose)-dependent pathway in the early response to DNA-damaging agents. J Biol Chem 2007, 282, (22), 16441-53.

87. Hong, Z.; Jiang, J.; Hashiguchi, K.; Hoshi, M.; Lan, L.; Yasui, A., Recruitment of mismatch repair proteins to the site of DNA damage in human cells. J Cell Sci $\mathbf{2 0 0 8 .}$

88. Zhang, X.; Morera, S.; Bates, P. A.; Whitehead, P. C.; Coffer, A. I.; Hainbucher, K.; Nash, R. A.; Sternberg, M. J.; Lindahl, T.; Freemont, P. S., Structure of an XRCC1 BRCT domain: a new protein-protein interaction module. Embo J 1998, 17, (21), 6404-11. 
89. Matsuoka, S.; Ballif, B. A.; Smogorzewska, A.; McDonald, E. R., 3rd; Hurov, K. E.; Luo, J.; Bakalarski, C. E.; Zhao, Z.; Solimini, N.; Lerenthal, Y.; Shiloh, Y.; Gygi, S. P.; Elledge, S. J., ATM and ATR substrate analysis reveals extensive protein networks responsive to DNA damage. Science 2007, 316, (5828), 1160-6.

90. Rush, J.; Moritz, A.; Lee, K. A.; Guo, A.; Goss, V. L.; Spek, E. J.; Zhang, H.; Zha, X. M.; Polakiewicz, R. D.; Comb, M. J., Immunoaffinity profiling of tyrosine phosphorylation in cancer cells. Nat Biotechnol 2005, 23, (1), 94-101. 
Table 1. Phosphopeptides detected by mass spectrometry analysis of tryptic digests of PARP-1 and PARG. The probability of correct peptide identification is reflected by either Sequest's XCorr and deltaCn ( $\mathrm{dCn})$ scores (MS/MS identifications) or the mass difference $\delta \mathrm{m}$ between the observed and theoretical peptide mass (MALDI-TOF MS observations). Ascore values are reported for phosphopeptides identified by MS/MS when there is more than one possible site of phosphorylation within the peptide. Ascore values lower than 20 indicate that the site of phosphorylation cannot be confidently assigned. In those cases: all the possible sites, given the observed MS/MS spectrum, are listed in the "Phosphorylated residue(s)" column separated by "or"; and the XCorr and deltaCn scores of the corresponding phosphopeptides are reported. When there are many deltaCn scores for a peptide, all values but the last thus correspond to XCorr differences between the possible phosphopeptides whereas the last value corresponds to the XCorr difference between the last possible phosphopeptide and the next Sequest peptide-spectrum match. See Supplementary information for detailed analysis of each peptide-spectrum match. 


\begin{tabular}{|c|c|c|c|c|c|c|}
\hline \multirow{2}{*}{ Kinase } & \multicolumn{2}{|l|}{ Phosphopeptide } & \multicolumn{3}{|c|}{$\begin{array}{c}\text { Identified by MS/MS } \\
\text { (LC-ESI-LTQ) }\end{array}$} & \multirow{2}{*}{$\begin{array}{c}\begin{array}{r}\text { Observed by MS } \\
\text { (MALDI-TOF) }\end{array} \\
\delta m \\
(D a)\end{array}$} \\
\hline & Sequence & $\begin{array}{l}\text { Phosphorylated } \\
\text { residue(s) }\end{array}$ & $\begin{array}{c}\text { Sequest } \\
\text { Xcorr }\end{array}$ & $\begin{array}{c}\text { Sequest } \\
\text { dCn }\end{array}$ & Ascore & \\
\hline \multicolumn{7}{|l|}{ PARP-1 } \\
\hline \multirow{6}{*}{$\begin{array}{l}\text { Endo- } \\
\text { genous }\end{array}$} & KCSESIPKDpSLR & $\mathrm{S}^{32}$ & - & - & - & -0.16 \\
\hline & KVCpSpTNDLK & $\mathrm{S}^{257}$ or $\mathrm{T}^{258}$ & $3.28,2.78$ & $0.15,0.90$ & 11.5 & - \\
\hline & KVCpSpTNDLKELLIFNK & $\mathrm{S}^{257}$ or $\mathrm{T}^{258}$ & $4.50,4.17$ & $0.07,0.80$ & 13.3 & - \\
\hline & KEWVpTPKEFR & $\mathrm{T}^{335}$ & - & - & - & -0.09 \\
\hline & $\begin{array}{l}\text { IFPPETSASVAApTPPPpSpTApSAPAAVNS } \\
\text { SASADKPLSNMK }\end{array}$ & $\mathrm{T}^{368}$ or $\mathrm{S}^{372}$ or $\mathrm{T}^{373}$ or $\mathrm{S}^{374}$ & $\begin{array}{l}4.35,4.34 \\
4.27,4.24\end{array}$ & $\begin{array}{c}0.003,0.02 \\
0.03,0.12\end{array}$ & 8.9 & - \\
\hline & GGpSDDSSKDPIDVNYEK & $S^{782}$ & 4.85 & 0.11 & 53.3 & -0.53 \\
\hline \multirow{10}{*}{ Erk1 } & MAIMVQpSPMFDGK & $S^{41}$ & 4.21 & 0.46 & N/A & - \\
\hline & NREELGFRPEYpSASQLK & $S^{177}$ & $2.89,2.89$ & $0.003,0.21$ & $\begin{array}{l}0 \text { (explained in } \\
\text { Suppl. data) }\end{array}$ & - \\
\hline & KVCpSpTNDLKELLIFNK & $\mathrm{S}^{257}$ or $\mathrm{T}^{258}$ & $3.85,3.65$ & $0.05,0.66$ & 0 & - \\
\hline & VCpSpTNDLKELLIFNK & $\mathrm{S}^{257}$ or $\mathrm{T}^{258}$ & $3.10,2.85$ & $0.08,0.49$ & 9.3 & - \\
\hline & $\begin{array}{l}\text { IFPPETSASVAApTPPPpSpTApSAPAAVNS } \\
\text { SASADKPLSNMK }\end{array}$ & $\mathrm{T}^{368}$ or $\mathrm{S}^{372}$ or $\mathrm{T}^{373}$ or $\mathrm{S}^{374}$ & $\begin{array}{l}4.30,4.18 \\
4.18,4.17\end{array}$ & $\begin{array}{l}0.03,0.03 \\
0.03,0.17\end{array}$ & 16.1 & - \\
\hline & LGGKLpTGpTANK & $\mathrm{T}^{420}$ or $\mathrm{T}^{422}$ & $3.62,3.04$ & $0.16,0.54$ & 14.4 & - \\
\hline & VVpSEDFLQDVSASTK & $S^{455}$ & 3.96 & 0.71 & 53.8 & - \\
\hline & GGAAVDPDSGLEHpSAHVLEK & $\mathrm{S}^{542}$ & 3.85 & 0.88 & 21.1 & - \\
\hline & KLpTVNPGTK & $\mathrm{T}^{656}$ & 1.96 & 0.77 & 22.9 & - \\
\hline & GGpSDDpSpSKDPIDVNYEK & $\mathrm{S}^{782}$ and $\left(\mathrm{S}^{785}\right.$ or $\left.\mathrm{S}^{786}\right)$ & $5.46,4.85$ & $0.11,0.26$ & $48.3,11.5$ & - \\
\hline \multirow{3}{*}{ РКС $\beta$} & SGAALpSKK & $\mathrm{S}^{504}$ & 1.51 & 0.67 & 75.5 & - \\
\hline & GQVKEEGINKpSEKR & $\mathrm{S}^{519}$ & 3.92 & 0.43 & N/A & - \\
\hline & KLpTVNPGTK & $T^{656}$ & 2.45 & 0.74 & 21.2 & - \\
\hline \multirow{2}{*}{ CaMK-II } & KVCpSpTNDLK & $\mathrm{S}^{257}$ or $\mathrm{T}^{258}$ & $2.24,2.05$ & $0.08,0.64$ & 0 & - \\
\hline & GGpSDDpSpSKDPIDVNYEK & $\mathrm{S}^{782}$ and $\left(\mathrm{S}^{785}\right.$ or $\left.\mathrm{S}^{786}\right)$ & $5.04,4.79$ & $0.05,0.13$ & $28.3,11.5$ & - \\
\hline \multirow{2}{*}{ JNK1 } & KVCpSpTNDLKELLIFNK & $\mathrm{S}^{257}$ or $\mathrm{T}^{258}$ & $5.29,4.97$ & $0.06,0.85$ & 13.5 & - \\
\hline & GGpSDDSSKDPIDVNYEK & $\mathrm{S}^{782}$ & 5.43 & 0.99 & 32.4 & - \\
\hline CDK5 & GGpSDDpSpSKDPIDVNYEK & $\mathrm{S}^{782}$ or $\mathrm{S}^{785}$ or $\mathrm{S}^{786}$ & $\begin{array}{l}4.56,4.12 \\
\quad 3.96\end{array}$ & $\begin{array}{l}0.10,0.13 \\
0.91\end{array}$ & 13.49 & - \\
\hline \multicolumn{7}{|l|}{ PARG } \\
\hline \multirow[t]{6}{*}{ CKII } & SPQNDDHpSDpTDSEENRDNQQFLTTVK & $\mathrm{S}^{197}$ and $\mathrm{T}^{199}$ & 4.93 & 0.11 & $23.2,29.9$ & - \\
\hline & QESCLGNSPPFEKEPSEPESPMDVDNSK & $\mathrm{S}^{298}$ & 4.97 & 0.10 & 26.3 & - \\
\hline & $\begin{array}{l}\text { SCDPGEDCASCQQDEIDVVPEpSPLpSDV } \\
\text { GSEDVGTGPK }\end{array}$ & $\mathrm{S}^{261}$ or $\mathrm{S}^{264}$ & $2.72,2.63$ & $0.03,0.11$ & 0 & - \\
\hline & $\begin{array}{l}\text { NSCQDpSEADEETSPGFDEQEDGSSSQTA } \\
\text { NKPSR }\end{array}$ & $S^{316}$ & 6.29 & 0.14 & 23.4 & - \\
\hline & LYPFIYHAVEpSCAEpTADHSGQR & $\mathrm{S}^{962}$ or $\mathrm{T}^{966}$ & $3.97,3.76$ & $0.05,0.10$ & 7.7 & - \\
\hline & HpTEQLLEpSEPQpTVTLVPEQFSNANIDR & $\mathrm{T}^{164}$ or $\mathrm{S}^{170}$ or $\mathrm{T}^{174}$ & $\begin{array}{l}3.50,3.50 \\
3.25\end{array}$ & $\begin{array}{l}0.00,0.01 \\
0.08\end{array}$ & 5.1 & - \\
\hline
\end{tabular}


Table 2. Prediction-aided interpretation of the phosphorylation sites identified by mass spectrometry. Each phosphorylation site is listed with corresponding kinase-specific predictions from Phoscan and NetphosK as described in Materials and Methods. The phosphorylation sites listed include the PARP-1 and PARG phosphoresidues identified in this study as well as those reported in the literature. The predicted phosphorylation potential for each phosphoresidue is also displayed (see Supplementary Table S1 for a complete score listing). 


\begin{tabular}{|c|c|c|c|c|c|c|c|c|c|c|c|}
\hline \multirow[t]{2}{*}{$\begin{array}{l}\text { Phospho-rylated } \\
\text { residue(s) }\end{array}$} & \multicolumn{7}{|c|}{$\begin{array}{l}\text { For each phosphorylation experiment: } \\
\text { Phosphorylation sites as revealed by either MS/MS } \\
\text { (black rectangle) or MALDI-TOF MS (gray } \\
\text { rectangle). («x» : reported in the litterature) }\end{array}$} & \multirow[t]{2}{*}{$\begin{array}{l}\text { Phoscan } \\
\text { kinase } \\
\text { prediction }\end{array}$} & \multirow[t]{2}{*}{$\begin{array}{l}\text { Netphosk } \\
\text { kinase } \\
\text { prediction }\end{array}$} & \multirow[t]{2}{*}{$\begin{array}{l}\text { Phospho- } \\
\text { rylation } \\
\text { potential }\end{array}$} & \multirow[t]{2}{*}{ References } \\
\hline & $\begin{array}{l}\text { Endo- } \\
\text { genous }\end{array}$ & Erk1 & CKII & PKC $\beta$ & $\begin{array}{c}\text { CaMK } \\
-I I\end{array}$ & $J N K 1$ & CDK5 & & & & \\
\hline \multicolumn{12}{|l|}{ PARP-1 } \\
\hline$S^{32}$ & & & & & & & & PKA, PKC & Not predicted & High & This study \\
\hline$S^{41}$ & & & & & & & & CDK, MAPK & CDK5, p38MAPK & Low & This study \\
\hline $\mathrm{S}^{177}$ & & & & & & & & Not predicted & Not predicted & Low & This study \\
\hline$S^{179}$ & $\mathbf{X}$ & & & & & & & ATM, DNA-PK & $\begin{array}{l}\text { ATM, DNA-PK, } \\
\text { CDC2 }\end{array}$ & Low & Matsuoka et $a l^{89}$ \\
\hline $\mathrm{S}^{257}$ or $\mathrm{T}^{258}$ & & & & & & & & $\begin{array}{l}\text { CaMK-II / Not } \\
\text { predicted }\end{array}$ & Not predicted / PKC & High / Low & This study \\
\hline $\mathrm{T}^{335}$ & & & & & & & & CDK, MAPK & CKII & High & This study \\
\hline $\begin{array}{l}\mathrm{T}^{368} \text { or } \mathrm{S}^{372} \text { or } \mathrm{T}^{373} \text { or } \\
\mathrm{S}^{375}\end{array}$ & & $\mathbf{X}$ & & & & & & $\begin{array}{l}\text { CDK, MAPK / } \\
\text { DNA-PK / MAPK / } \\
\text { Not predicted }\end{array}$ & $\begin{array}{l}\text { GSK3, CDC2, } \\
\text { CDK5, p38MAPK / } \\
\text { Not predicted / Not } \\
\text { predicted / Not } \\
\text { predicted }\end{array}$ & $\begin{array}{l}\text { High / High / Low / } \\
\text { Low }\end{array}$ & $\begin{array}{l}\text { This study and } \\
\text { Kauppinen } \text { et al. }\end{array}$ \\
\hline $\mathrm{T}^{420}$ or $\mathrm{T}^{422}$ & & & & & & & & $\mathrm{PKC} / \mathrm{PKC}$ & $\mathrm{PKC} / \mathrm{PKC}$ & Low / Low & This study \\
\hline$S^{455}$ & & & & & & & & PKA, CKII, AMP-K & RSK & Low & This study \\
\hline$S^{504}$ & & & & & & & & PKC & PKC & High & This study \\
\hline$S^{519}$ & & & & & & & & PKC & PKC & High & This study \\
\hline $\mathrm{S}^{542}$ & & & & & & & & Not predicted & Not predicted & Low & This study \\
\hline $\mathrm{T}^{656}$ & & & & & & & & PKA, PKC & PKC & Low & This study \\
\hline$Y^{775}$ & $\mathbf{X}$ & & & & & & & Not predicted & Not predicted & Low & Rush et al. ${ }^{90}$ \\
\hline$S^{782}$ & & & & & & & & $\begin{array}{l}\text { PKA, CKII, CaMK- } \\
\text { II, MAPKAP-2 }\end{array}$ & PKA & High & This study \\
\hline \multirow[t]{2}{*}{$\mathrm{S}^{782}$ and $\left(\mathrm{S}^{785}\right.$ or $\left.\mathrm{S}^{786}\right)$} & & & & & & & & $\begin{array}{l}\text { PKA, CKII, CaMK- } \\
\text { II, MAPKAP-2 / Not } \\
\text { predicted / Not } \\
\text { predicted }\end{array}$ & $\begin{array}{l}\mathrm{PKA} / \mathrm{CDC} 2 / \\
\mathrm{CDC} 2, \mathrm{CKII}\end{array}$ & High / High / High & This study \\
\hline & & & & & & & & $\begin{array}{l}\text { PKA, CKII, CaMK- } \\
\text { II, MAPKAP-2 / Not } \\
\text { predicted / Not } \\
\text { predicted }\end{array}$ & $\begin{array}{l}\mathrm{PKA} / \mathrm{CDC} 2 / \\
\mathrm{CDC} 2, \mathrm{CKII}\end{array}$ & High / High / High & This study \\
\hline \multicolumn{12}{|l|}{ PARG } \\
\hline$S^{22}$ & $\mathbf{X}$ & & & & & & & CDK, MAPK & CDK5, GSK3 & High & Beausoleil et al. ${ }^{49}$ \\
\hline $\mathrm{S}^{137}$ & $\mathbf{x}$ & & & & & & & MAPK & CDK5, GSK3 & High & Villen et al $^{48}$ \\
\hline $\mathrm{S}^{139}$ & $\mathbf{x}$ & & & & & & & $\begin{array}{l}\text { PKC, CaMK-II, } \\
\text { DNA-PK }\end{array}$ & PKC & High & Imami et al. ${ }^{50}$ \\
\hline $\mathrm{T}^{164}$ or $\mathrm{S}^{170}$ or $\mathrm{T}^{174}$ & & & & & & & & $\begin{array}{l}\text { CKII / Not predicted } \\
\text { / Not Predicted }\end{array}$ & $\begin{array}{l}\text { CKII / CKI, CDC2 / } \\
\text { Not predicted }\end{array}$ & Low / High / Low & This study \\
\hline $\mathrm{S}^{197}$ and $\mathrm{T}^{199}$ & & & & & & & & CKII / CKII & CKII / CKII & High / High & This study \\
\hline$S^{261}$ and $S^{264}$ & $\mathbf{X}$ & & & & & & & MAPK, ERK / CKII & $\begin{array}{l}\text { P38MAPK,GSK3 / } \\
\text { CKI }\end{array}$ & High / High & Villen et al. ${ }^{48}$ \\
\hline $\mathrm{S}^{261}$ or $\mathrm{S}^{264}$ & & & & & & & & MAPK, ERK / CKII & P38MAPK,GSK3 / & High / High & This study \\
\hline
\end{tabular}




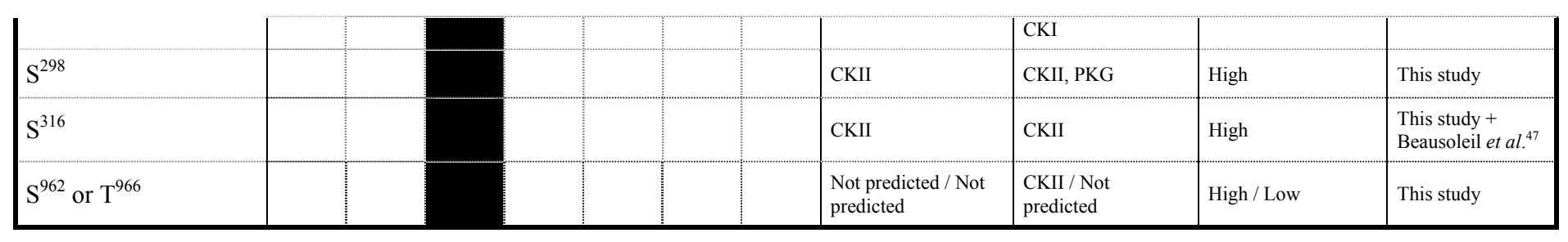


Table of contents

Phosphorylation is a very common posttranslational modification event known to modulate a wide range of biological responses. Beyond the regulation of protein activity, the interrelation of phosphorylation with other posttranslational mechanisms is responsible for the control of diverse signaling pathways. Several observations suggest that phosphorylation of poly(ADP-ribose) polymerase 1 (PARP-1) regulates its activity.

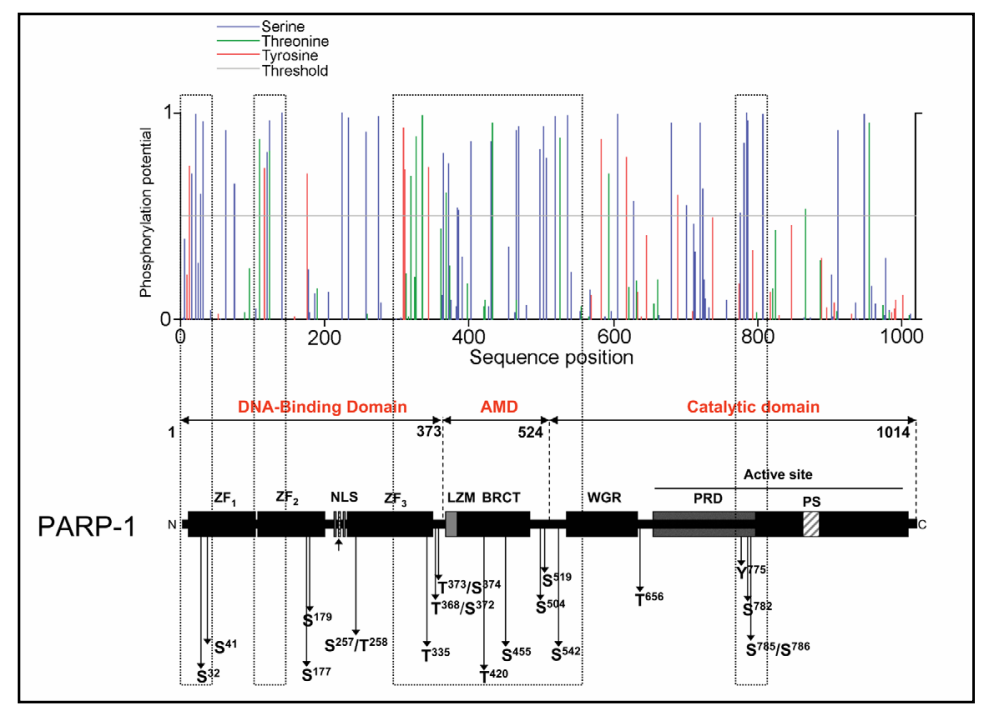
There is also accumulating evidence to suggest the establishment of phosphorylationdependent assembly of PARP-1-associated multi-protein complexes. Although it is relatively straightforward to demonstrate phosphorylation of a defined target, identification of the actual amino acids involved still represents a technical challenge for many laboratories. Using a combination of bioinformatics-based predictions tools for generic and kinase-specific phosphorylation sites, in vitro phosphorylation assays and mass spectrometry analysis, we investigated the phosphorylation profile of PARP-1 and poly(ADP-ribose) glycohydrolase (PARG), two major enzymes responsible for poly(ADPribose) turnover. Mass spectrometry analysis revealed the phosphorylation of several serine/threonine residues within important regulatory domains and motifs of both enzymes. Using in vivo microirradiation-induced DNA damage, we show that altered phosphorylation at specific sites can modify the dynamics of assembly and disassembly of PARP-1 at sites of DNA damage. By documenting and annotating a collection of known and newly identified phosphorylation sites, this targeted proteomics study significantly advances our understanding of the roles of phosphorylation in the regulation of PARP-1 and PARG. 


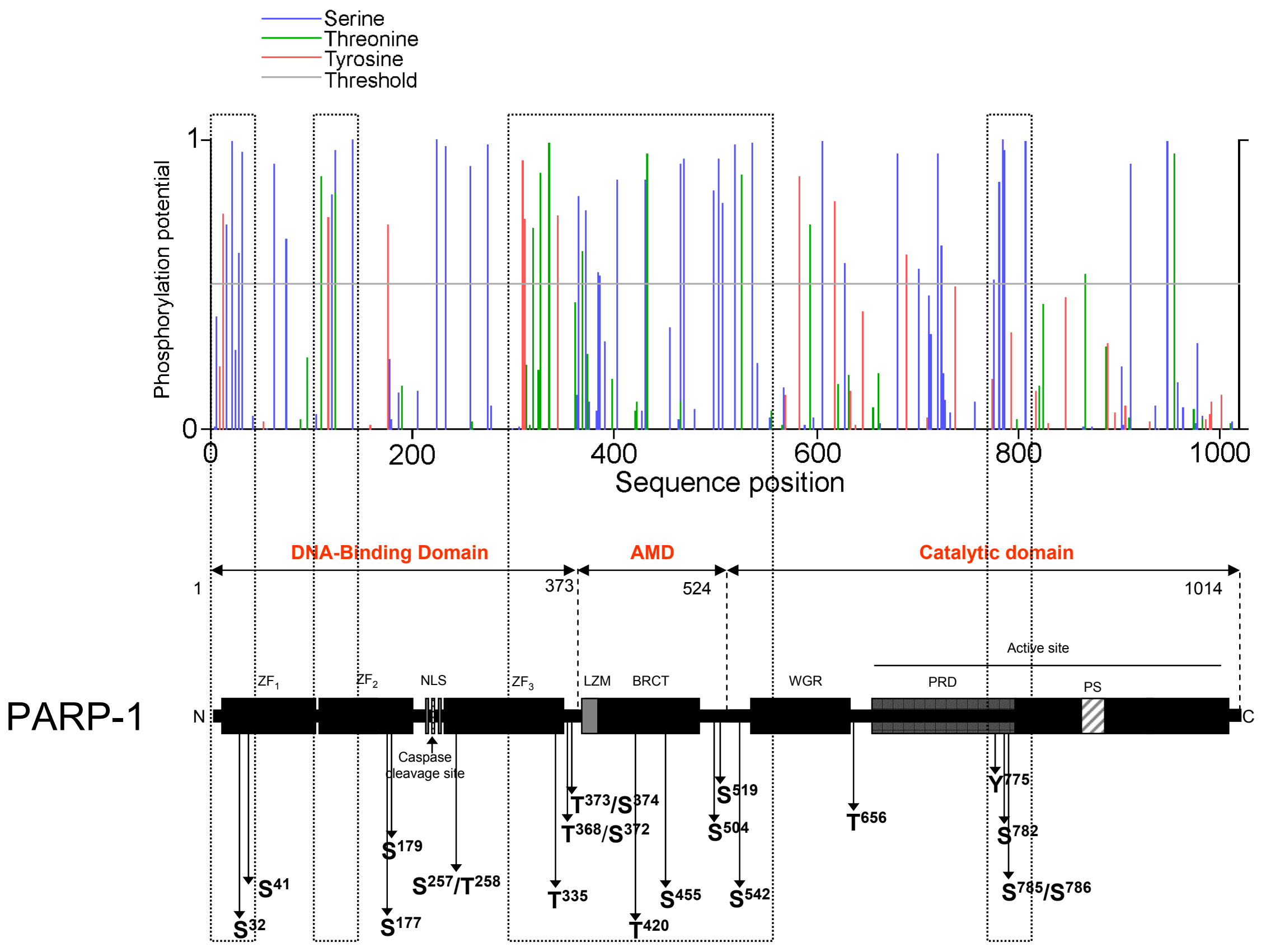

Figure 1A 


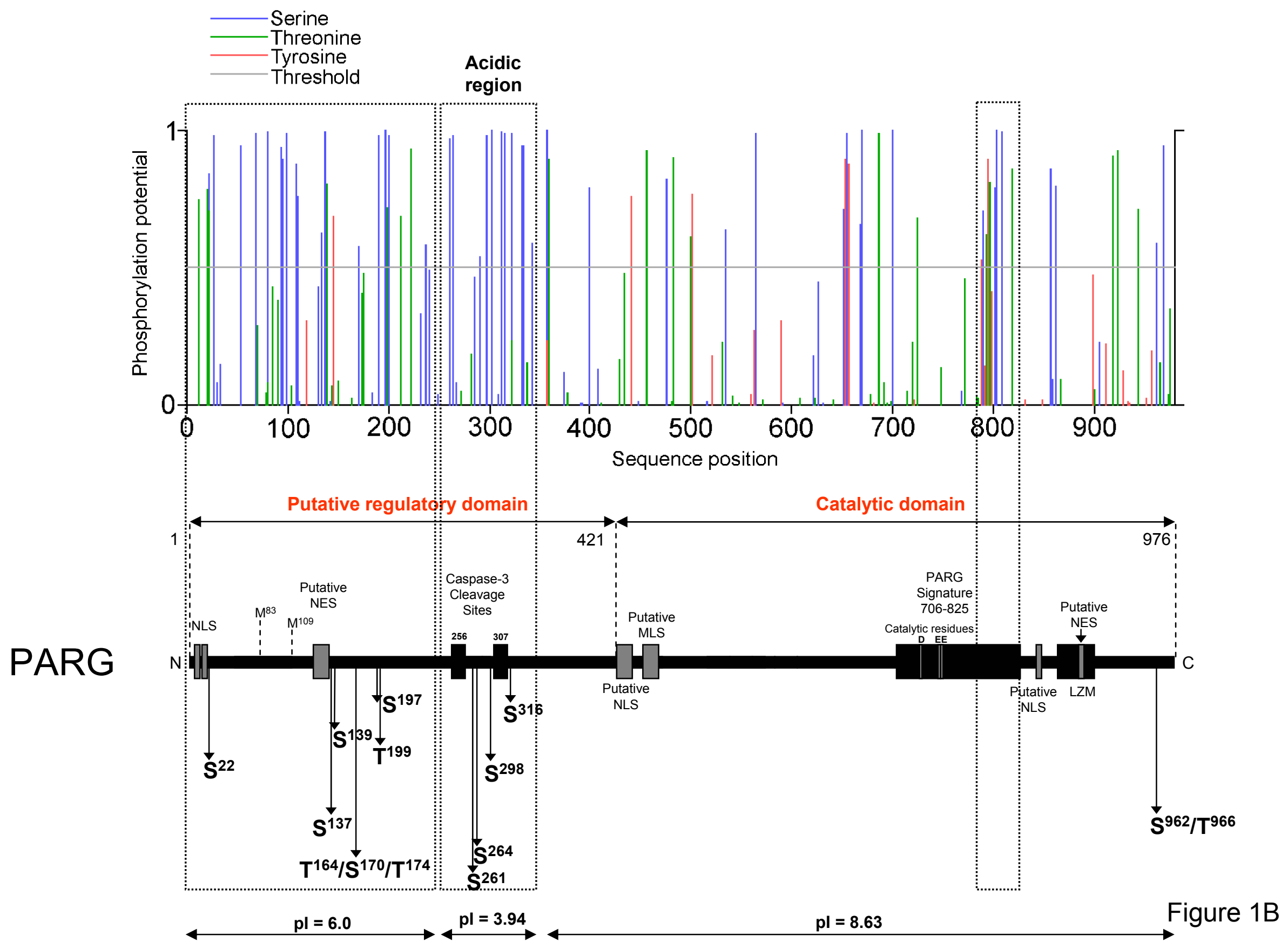


A

PARP-1

ATP

PKC $\alpha$

PKC $\beta$

$+$

$+$

+
+
+

+
+
+

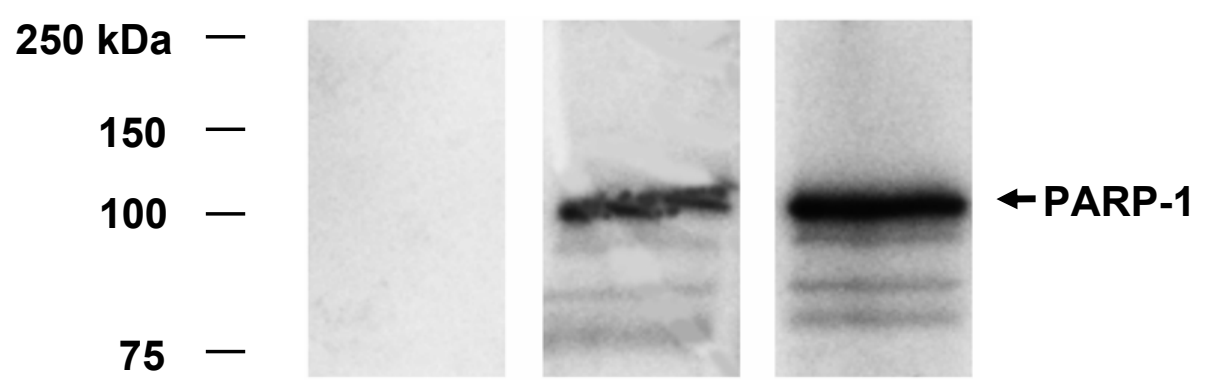

B

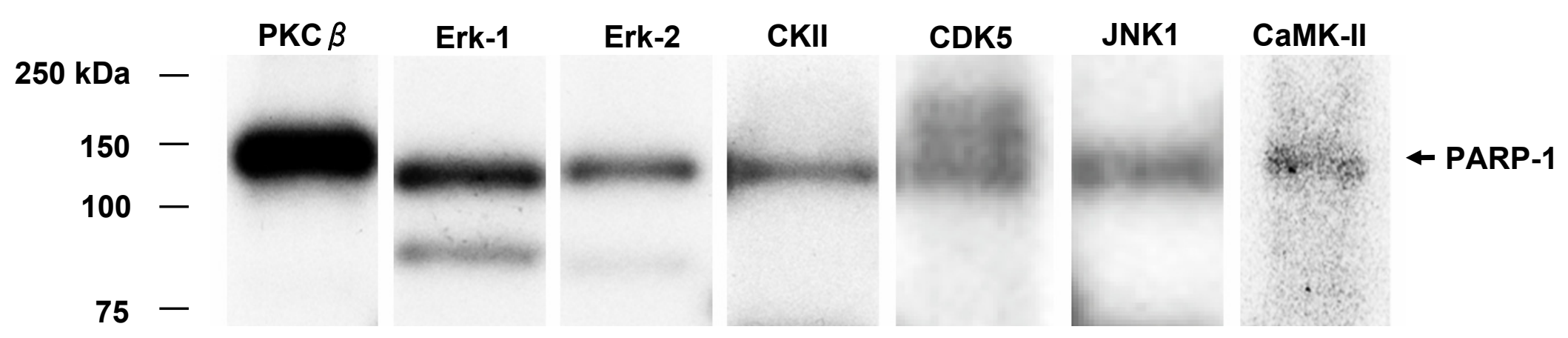

C

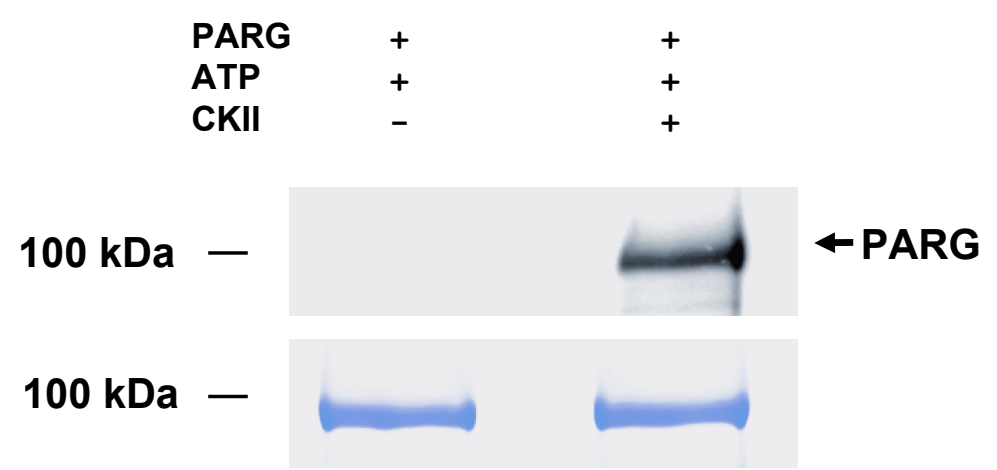

Figure 2 


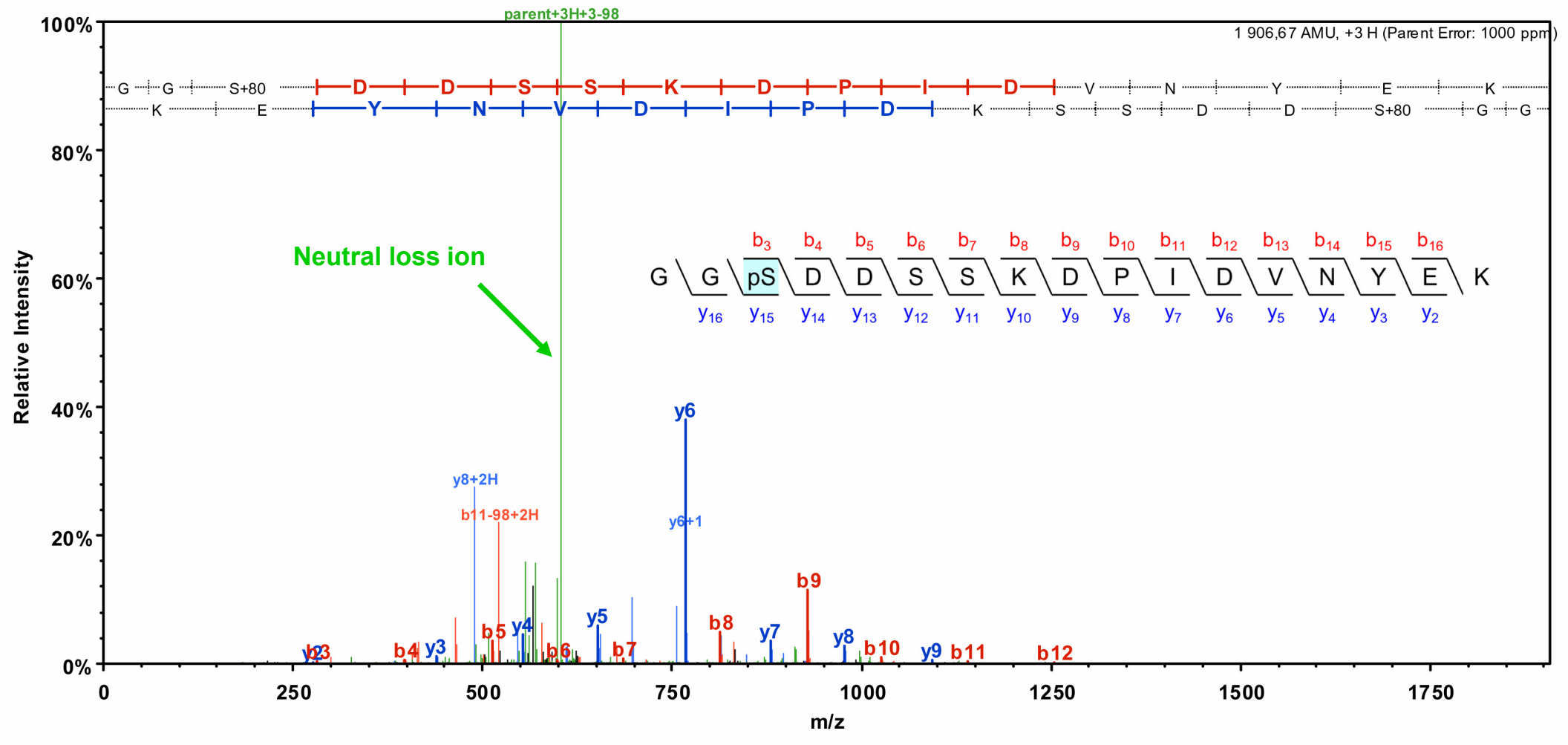

\begin{tabular}{|c|c|c|c|c|c|c|c|c|c|c|}
\hline B & B Ions & $\mathrm{B}+2 \mathrm{H}$ & $\mathrm{B}-\mathrm{NH} 3$ & $\mathrm{~B}-\mathrm{H} 2 \mathrm{O}$ & AA & $Y$ Ions & $\mathrm{Y}+2 \mathrm{H}$ & $\mathrm{Y}-\mathrm{NH} 3$ & $\mathrm{Y}-\mathrm{H} 2 \mathrm{O}$ & $Y$ \\
\hline 1 & 58,0 & & & & G & 1905,8 & 953,4 & 1888,7 & 1887,8 & 17 \\
\hline 2 & 115,1 & & & & G & 1848,8 & 924,9 & 1831,7 & 1830,7 & 16 \\
\hline 3 & 282,0 & & & 264,0 & $5+80$ & 1791,7 & 896.4 & 1774,7 & 1773,7 & 15 \\
\hline 4 & 397.1 & & & 379,1 & D & 1624,7 & 812,9 & 1607,7 & 1606,7 & 14 \\
\hline 5 & 512.1 & & & 494,1 & D & 1509,7 & 755,4 & 1492,7 & 1491,7 & 13 \\
\hline 6 & 599.1 & 300.1 & & 581,1 & S & 1394,7 & 697,8 & 1377,7 & 1376,7 & 12 \\
\hline 7 & 686.2 & 343,6 & & 668.2 & S & 1307.6 & 654.3 & 1290,6 & 1289.6 & 11 \\
\hline 8 & 814.3 & 407.6 & 797,2 & 796.3 & K & 1220,6 & 610.8 & 1203,6 & 1202,6 & 10 \\
\hline 9 & 929.3 & 465.1 & 912,3 & 911,3 & D & 1092,5 & 546,8 & 1075,5 & 1074,5 & 9 \\
\hline 10 & 1026.3 & 513,7 & 1009.3 & 1008,3 & $\mathbf{P}$ & 977.5 & 489,3 & 960.5 & 959.5 & 8 \\
\hline 11 & 1139.4 & 570,2 & 1122,4 & 1121.4 & I & 880,4 & 440,7 & 863,4 & 862,4 & 7 \\
\hline 12 & 1254,5 & 627.7 & 1237,4 & 1236,4 & D & 767.4 & 384,2 & 750.3 & 749,3 & 6 \\
\hline 13 & 1353,5 & 677,3 & 1336,5 & 1335,5 & V & 652,3 & & 635,3 & 634,3 & 5 \\
\hline 14 & 1467,6 & 734,3 & 1450,5 & 1449,6 & $\mathbf{N}$ & 553.3 & & 536.2 & 535,3 & 4 \\
\hline 15 & 1630,6 & 815,8 & 1613,6 & 1612,6 & $\mathbf{Y}$ & 439,2 & & 422,2 & 421,2 & 3 \\
\hline 16 & 1759,7 & 880.3 & 1742,6 & 1741,7 & $E$ & 276.2 & & 259,1 & 258,1 & 2 \\
\hline 17 & 1905,8 & 953,4 & 1888,7 & 1887,8 & K & 147,1 & & 130,1 & & 1 \\
\hline
\end{tabular}

Figure 3 

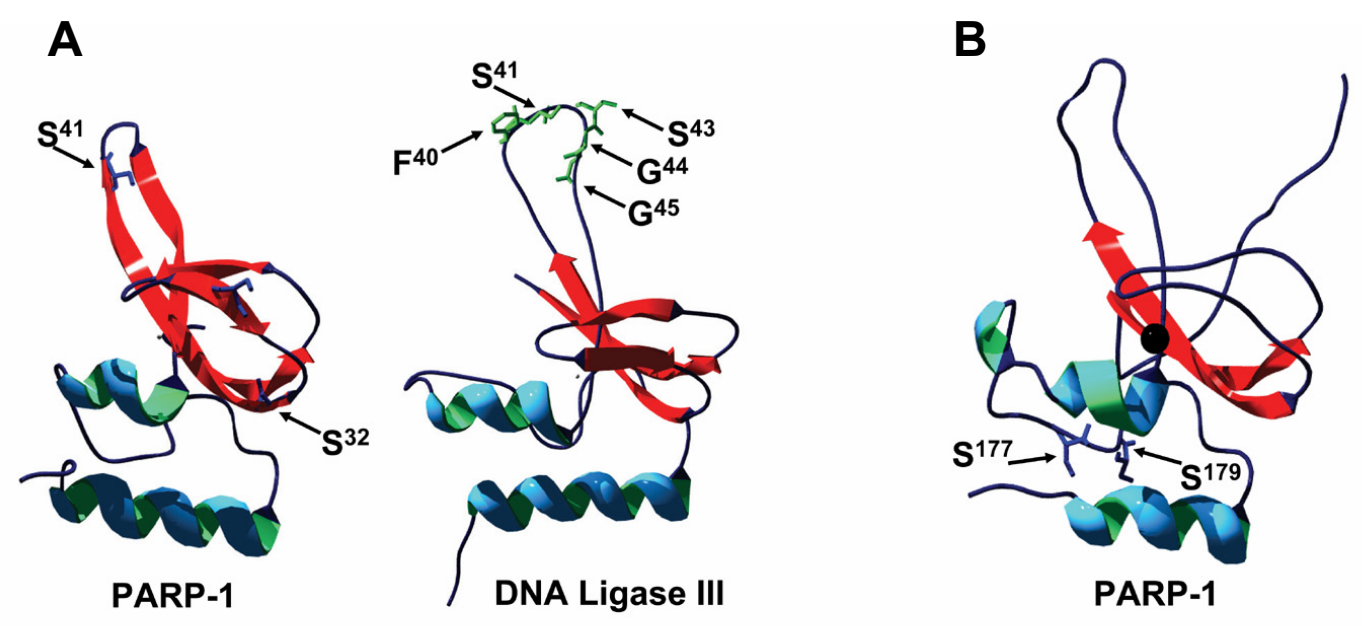

C
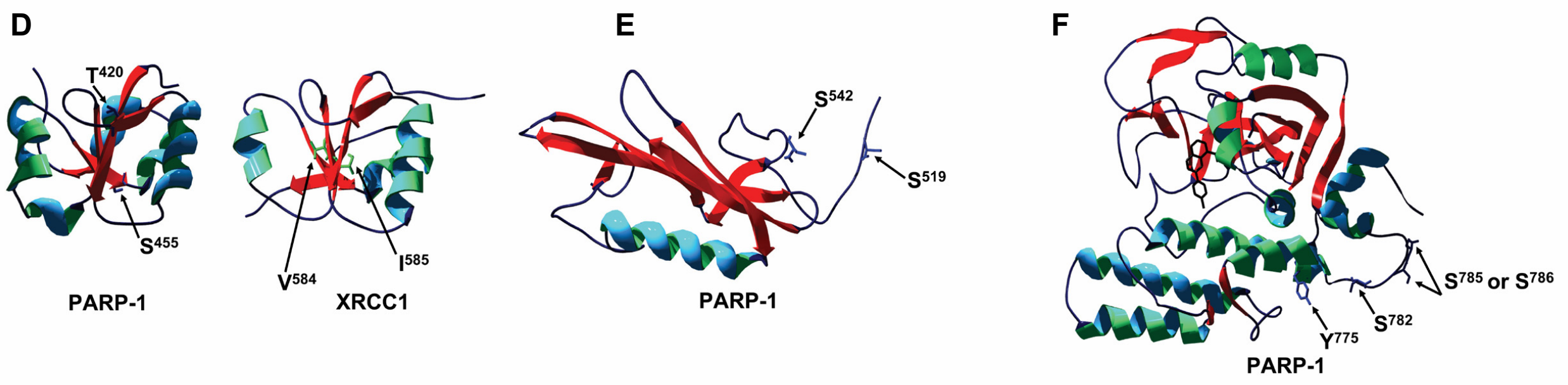

Figure 4 

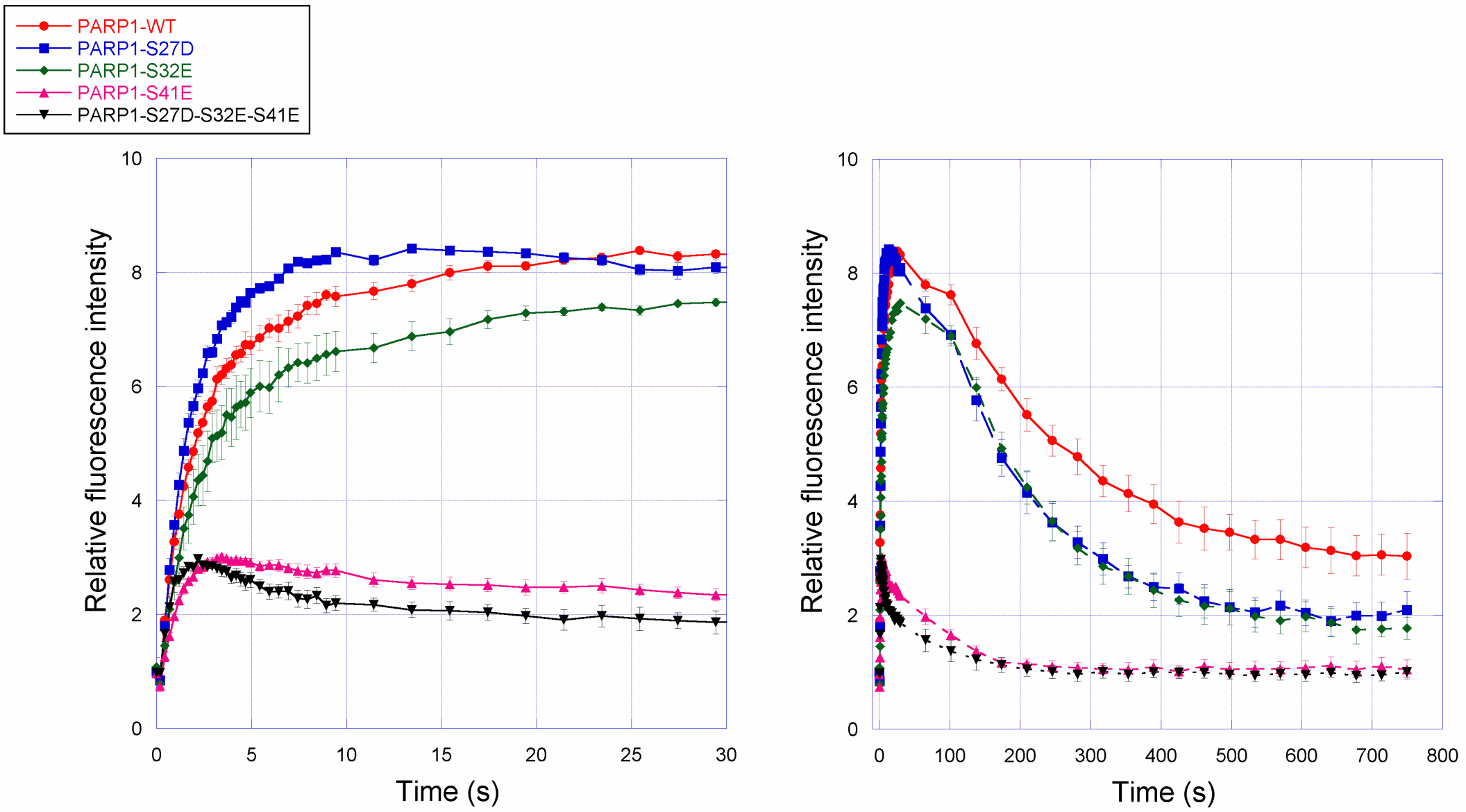

Figure 5 


\section{Supplementary mass spectrometric data}

\section{Legend for MS/MS spectra's fragmentation tables}

- $\quad$ Red background: observed singly-charged (+1) and doubly-charged (+2) b ions.

- Blue background: observed +1 and +2 y ions.

- $\quad$ Green background: $b$ and y ions with either a water (-H2O) or ammonia (-NH3) loss.

- "C+57": carboxyamidomethylated cysteine

- "M+16": Oxidized methionine.

- $\quad$ "S+80", “ $T+80$ ", " $Y+80$ ": phosphorylated serine, threonine, tyrosine.

\section{Legend for MS/MS spectra}

\section{Peptide sequence:}

- $\quad$ The top line corresponds to $b$ ions and the second line to $y$ ions.

- When two consecutive singly $\boldsymbol{b}(\boldsymbol{y})$ ions are observed, the amino acid corresponding to the mass difference is shown in red (blue).

\section{MS/MS spectrum:}

- Phosphate neutral loss is flagged by "-98".

- $\quad$ Red lines correspond to observed +1 and $+2 \boldsymbol{b}$ ions, and blue lines to observed +1 and $+2 \boldsymbol{y}$ ions.

- $\quad \boldsymbol{b}$ and $\boldsymbol{y}$ ions with a loss of either a water (-H2O) ammonia (-NH3), or phosphate (-H3PO4) are depicted by green vertical lines. The parent (precursor) ion as well as a and $\mathbf{x}$ ions, with or without loss, are also depicted as green vertical lines.

- $\quad$ " $+2 H$ " and " $+3 H$ " indicate doubly and triply charged ions respectively.

- "+1", “+2", and "+3" indicates a match to the second, third, or fourth peak of the theoretical isotopic envelope of the fragment ion. The $L T Q$ does not resolve the isotopic envelope of the fragment ions and, in the case of heavy fragments, the centroided (average) mass can be significantly higher than the monoisotopic mass and lead to a match 1 to 3 Da higher than the monoisotopic mass.

- $\quad$ Black vertical lines indicate unidentified MS/MS fragments. 


\section{Please use the hyperlink in the "Sequence" and " $\delta m$ " columns to directly access the peptide's MS supplementary data}

\begin{tabular}{|c|c|c|c|c|c|c|}
\hline \multirow{2}{*}{ Kinase } & \multicolumn{2}{|l|}{ Phosphopeptide } & \multicolumn{3}{|c|}{$\begin{array}{l}\text { Identified by MS/MS } \\
\quad \text { (LC-ESI-LTQ) }\end{array}$} & \multirow{2}{*}{$\begin{array}{c}\begin{array}{c}\text { Observed by MS } \\
\text { (MALDI-TOF) }\end{array} \\
\delta m \\
(D a)\end{array}$} \\
\hline & Sequence & $\begin{array}{l}\text { Phosphorylated } \\
\text { residue(s) }\end{array}$ & $\begin{array}{c}\text { Sequest } \\
\text { Xcorr }\end{array}$ & $\begin{array}{l}\text { Sequest } \\
\text { dCn }\end{array}$ & Ascore & \\
\hline \multicolumn{7}{|l|}{ PARP-1 } \\
\hline \multirow{6}{*}{$\begin{array}{l}\text { Endo- } \\
\text { genous }\end{array}$} & KCSESIPKDpSLR & $\mathrm{S}^{32}$ & - & - & - & -0.16 \\
\hline & KVCpSpTNDLK & $\mathrm{S}^{257}$ or $\mathrm{T}^{258}$ & $3.28,2.78$ & $0.15,0.90$ & 11.5 & - \\
\hline & KVCpSpTNDLKELLIFNK & $\mathrm{S}^{257}$ or $\mathrm{T}^{258}$ & $4.50,4.17$ & $0.07,0.80$ & 13.3 & - \\
\hline & KEWVpTPKEFR & $\mathrm{T}^{335}$ & - & - & - & -0.09 \\
\hline & $\begin{array}{l}\text { IFPPETSASVAApTPPPpSpTApSAPAAVNS } \\
\text { SASADKPLSNMK }\end{array}$ & $\mathrm{T}^{368}$ or $\mathrm{S}^{372}$ or $\mathrm{T}^{373}$ or $\mathrm{S}^{374}$ & $\begin{array}{l}4.35,4.34 \\
4.27,4.24\end{array}$ & $\begin{array}{c}0.003,0.02 \\
0.03,0.12\end{array}$ & 8.9 & - \\
\hline & GGpSDDSSKDPIDVNYEK & $S^{782}$ & 4.85 & 0.11 & 53.3 & -0.53 \\
\hline \multirow{10}{*}{ Erk1 } & MAIMVQpSPMFDGK & $S^{41}$ & 4.21 & 0.46 & N/A & - \\
\hline & NREELGFRPEYpSASQLK & $S^{177}$ & $2.89,2.89$ & $0.003,0.21$ & $\begin{array}{l}0 \text { (explained in } \\
\text { Suppl. data) }\end{array}$ & - \\
\hline & KVCpSpTNDLKELLIFNK & $\mathrm{S}^{257}$ or $\mathrm{T}^{258}$ & $3.85,3.65$ & $0.05,0.66$ & 0 & - \\
\hline & VCpSpTNDLKELLIFNK & $\mathrm{S}^{257}$ or $\mathrm{T}^{258}$ & $3.10,2.85$ & $0.08,0.49$ & 9.3 & - \\
\hline & $\begin{array}{l}\text { IFPPETSASVAApTPPPpSpTApSAPAAVNS } \\
\text { SASADKPLSNMK }\end{array}$ & $\mathrm{T}^{368}$ or $\mathrm{S}^{372}$ or $\mathrm{T}^{373}$ or $\mathrm{S}^{374}$ & $\begin{array}{l}4.30,4.18 \\
4.18,4.17\end{array}$ & $\begin{array}{l}0.03,0.03 \\
0.03,0.17\end{array}$ & 16.1 & - \\
\hline & LGGKLpTGpTANK & $\mathrm{T}^{420}$ or $\mathrm{T}^{422}$ & $3.62,3.04$ & $0.16,0.54$ & 14.4 & - \\
\hline & VVpSEDFLQDVSASTK & $S^{455}$ & 3.96 & 0.71 & 53.8 & - \\
\hline & GGAAVDPDSGLEHpSAHVLEK & $S^{542}$ & 3.85 & 0.88 & 21.1 & - \\
\hline & KLpTVNPGTK & $\mathrm{T}^{656}$ & 1.96 & 0.77 & 22.9 & - \\
\hline & GGpSDDpSpSKDPIDVNYEK & $\mathrm{S}^{782}$ and $\left(\mathrm{S}^{785}\right.$ or $\left.\mathrm{S}^{786}\right)$ & $5.46,4.85$ & $0.11,0.26$ & $48.3,11.5$ & - \\
\hline \multirow{3}{*}{ PKC $\beta$} & SGAALpSKK & $S^{504}$ & 1.51 & 0.67 & 75.5 & - \\
\hline & GQVKEEGINKpSEKR & $S^{519}$ & 3.92 & 0.43 & N/A & - \\
\hline & KLpTVNPGTK & $\mathrm{T}^{656}$ & 2.45 & 0.74 & 21.2 & - \\
\hline \multirow{2}{*}{ CaMK-II } & KVCpSpTNDLK & $\mathrm{S}^{257}$ or $\mathrm{T}^{258}$ & $2.24,2.05$ & $0.08,0.64$ & 0 & - \\
\hline & GGpSDDpSpSKDPIDVNYEK & $\mathrm{S}^{782}$ and $\left(\mathrm{S}^{785}\right.$ or $\left.\mathrm{S}^{786}\right)$ & $5.04,4.79$ & $0.05,0.13$ & $28.3,11.5$ & - \\
\hline \multirow{2}{*}{ JNK1 } & KVCpSpTNDLKELLIFNK & $\mathrm{S}^{257}$ or $\mathrm{T}^{258}$ & $5.29,4.97$ & $0.06,0.85$ & 13.5 & - \\
\hline & GGpSDDSSKDPIDVNYEK & $S^{782}$ & 5.43 & 0.99 & 32.4 & - \\
\hline CDK5 & GGpSDDpSpSKDPIDVNYEK & $\mathrm{S}^{782}$ or $\mathrm{S}^{785}$ or $\mathrm{S}^{786}$ & $\begin{array}{l}\text { 4.56, } 4.12 \\
3.96\end{array}$ & $\begin{array}{l}0.10,0.13 \\
0.91\end{array}$ & 13.49 & - \\
\hline \multicolumn{7}{|l|}{ PARG } \\
\hline \multirow[t]{6}{*}{ CKII } & SPQNDDHpSDpTDSEENRDNQQFLTTVK & $\mathrm{S}^{197}$ and $\mathrm{T}^{199}$ & 4.93 & 0.11 & 23.2, 29.9 & - \\
\hline & QESCLGNSPPFEKEPSEPESPMDVDNSK & $\mathrm{S}^{298}$ & 4.97 & 0.10 & 26.3 & - \\
\hline & $\begin{array}{l}\text { SCDPGEDCASCQQDEIDVVPEpSPLpSDV } \\
\text { GSEDVGTGPK }\end{array}$ & $\mathrm{S}^{261}$ or $\mathrm{S}^{264}$ & $2.72,2.63$ & $0.03,0.11$ & 0 & - \\
\hline & $\begin{array}{l}\text { NSCQDpSEADEETSPGFDEQEDGSSSQTA } \\
\text { NKPSR }\end{array}$ & $S^{316}$ & 6.29 & 0.14 & 23.4 & - \\
\hline & LYPFIYHAVEpSCAEpTADHSGQR & $\mathrm{S}^{962}$ or $\mathrm{T}^{966}$ & $3.97,3.76$ & $0.05,0.10$ & 7.7 & - \\
\hline & HpTEQLLEpSEPQpTVTLVPEQFSNANIDR & $\mathrm{T}^{164}$ or $\mathrm{S}^{170}$ or $\mathrm{T}^{174}$ & $\begin{array}{l}3.50,3.50 \\
3.25\end{array}$ & $\begin{array}{l}0.00,0.01 \\
0.08\end{array}$ & 5.1 & - \\
\hline
\end{tabular}




\section{Supplementary mass spectrometric data:}

\section{PARP-1, endogenous phosphorylation}

Spectrum Parp_TiO2_20061213.4843.4843.2.dta (charge state: +2)

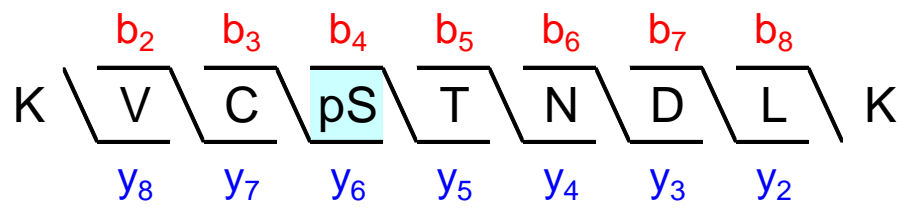

Sequest: $\mathrm{XCorr}=3.28, \mathrm{dCn} 1=0.15$ (between $1^{\text {st }}$ and $2^{\text {nd }}$ best matches), $\mathrm{dCn} 2=0.90$ (between $2^{\text {nd }}$ and $3^{\text {rd }}$ best matches).

Ascore $=11.51$.

Sequest's second best match with XCorr $=2.78$ is to the same peptide sequence but with an alternative phosphorylation site: KVCSPTNDLK.

The weak Ascore indicates that, given the observed MS/MS spectrum, the phosphopeptide identified could either be Sequest's first or second best match (Nat Biotechnol. 2006;24(10):1285-92).

\section{MS/MS spectrum's fragmentation table}

\begin{tabular}{|c|c|c|c|c|c|c|c|c|c|c|}
\hline B & B Ions & $\mathrm{B}+2 \mathrm{H}$ & $\mathrm{B}-\mathrm{NH} 3$ & $\mathrm{~B}-\mathrm{H} 2 \mathrm{O}$ & AA & $Y$ Ions & $\mathrm{Y}+2 \mathrm{H}$ & $\mathrm{Y}-\mathrm{NH} 3$ & $\mathrm{Y}-\mathrm{H} 2 \mathrm{O}$ & $Y$ \\
\hline 1 & 129,1 & 65.1 & 112,1 & & $K$ & 1144,5 & 572,8 & 1127,5 & 1126,5 & 9 \\
\hline 2 & 228.2 & 114,6 & 211,1 & & V & 1016.4 & 508.7 & 999,4 & 998,4 & 8 \\
\hline 3 & 388.2 & 194,6 & 371.2 & & $C+57$ & 917.3 & 459.2 & 900,3 & 899,3 & 7 \\
\hline 4 & 555.2 & 278.1 & 538.2 & 537,2 & $S+80$ & 757.3 & 379.2 & 740,3 & 739,3 & 6 \\
\hline 5 & 656.2 & 328.6 & 639.2 & 638,2 & $\mathbf{T}$ & 590.3 & & 573,3 & 572,3 & 5 \\
\hline 6 & 770.3 & 385,6 & 753.3 & 752,3 & $\mathrm{~N}$ & 489.3 & & 472.2 & 471,3 & 4 \\
\hline 7 & 885.3 & 443,2 & 868.3 & $\mathbf{8 6 7 . 3}$ & D & 375.2 & & 358.2 & 357.2 & 3 \\
\hline 8 & 998.4 & 499.7 & 981.4 & 980,4 & L & 260.2 & & 243.2 & & 2 \\
\hline 9 & 1144.5 & 572,8 & 1127.5 & 1126.5 & K & 147,1 & & 130,1 & & 1 \\
\hline
\end{tabular}




\section{MS/MS spectrum}

\section{Full-range}

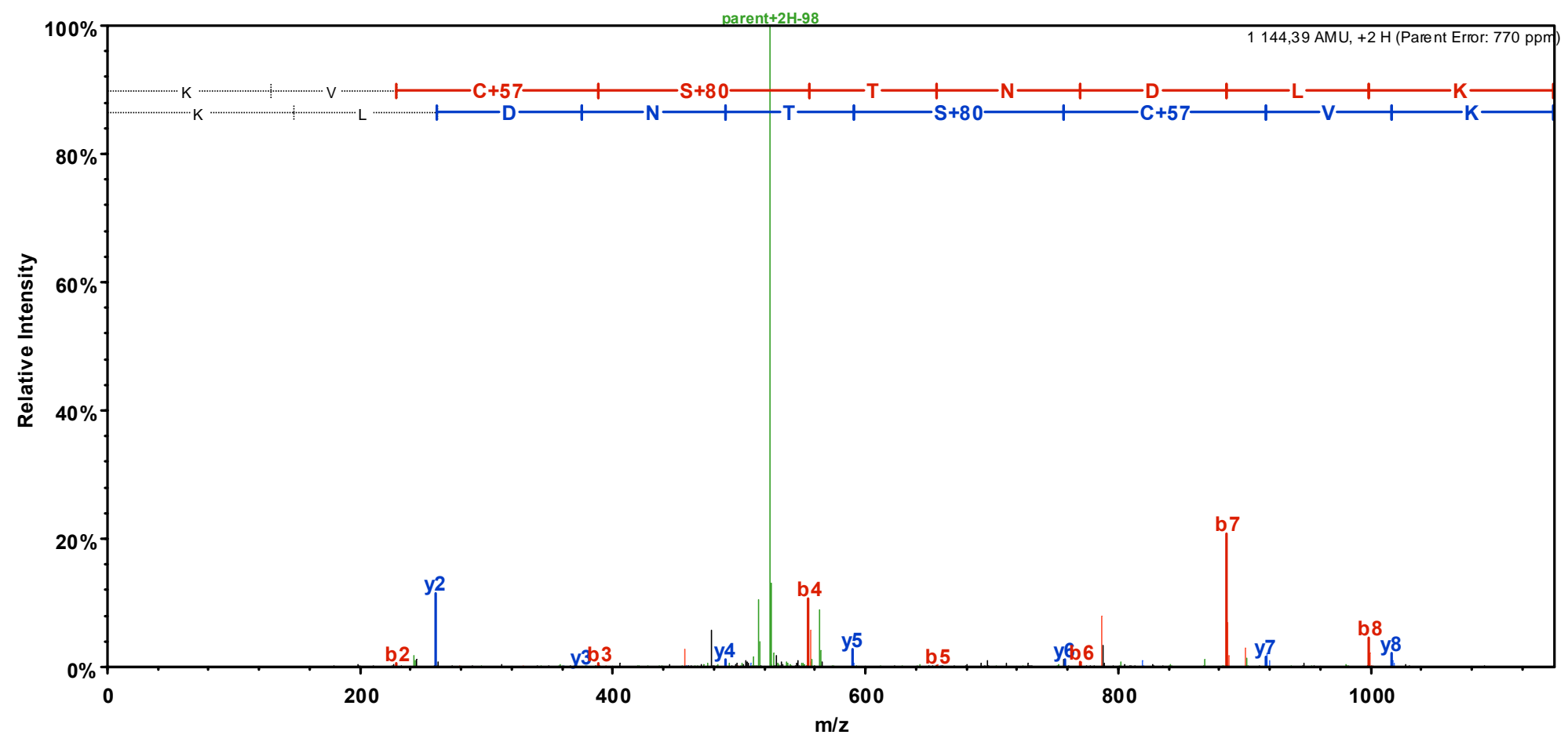

\section{$\underline{\text { Close to precursor }}$}

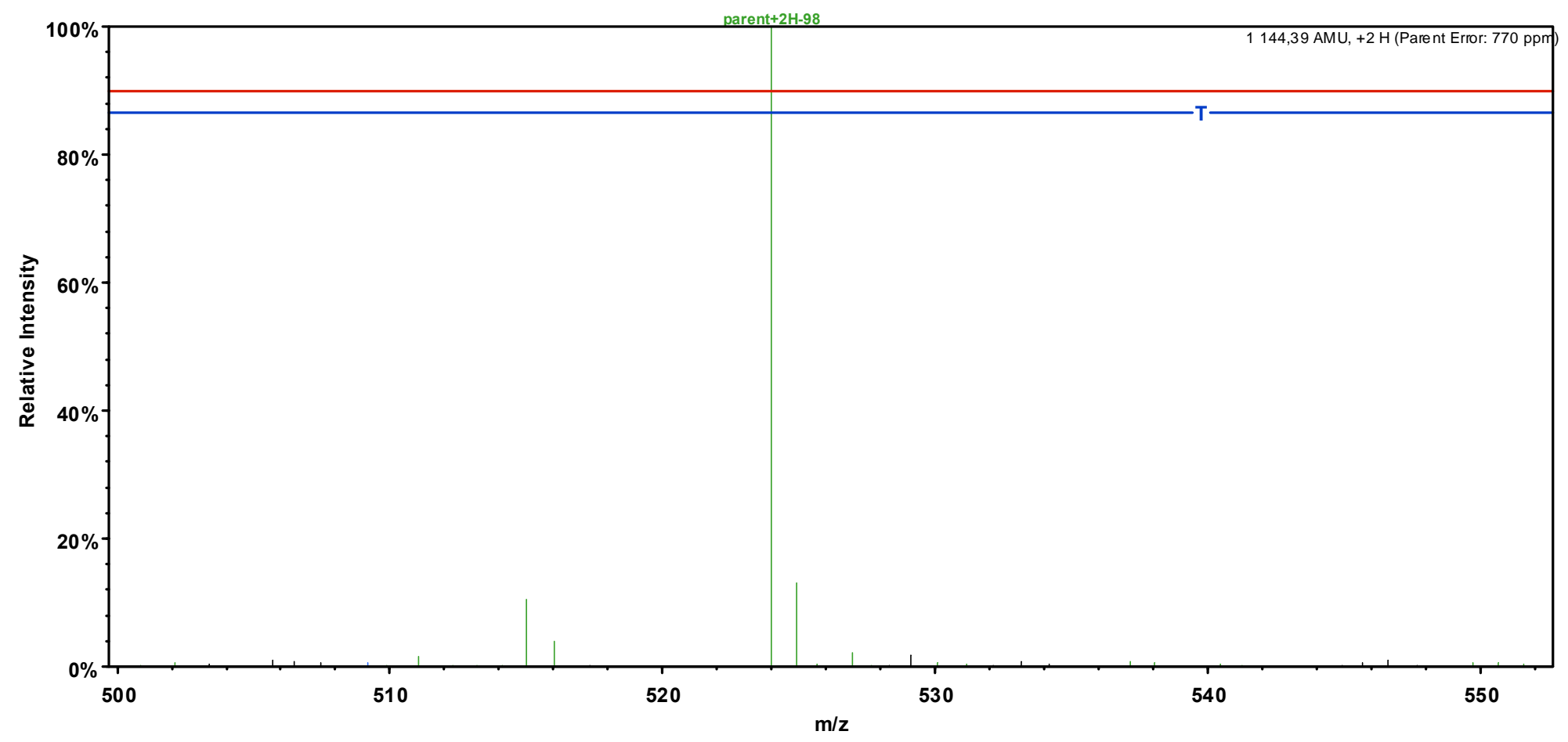




\section{Left of precursor}

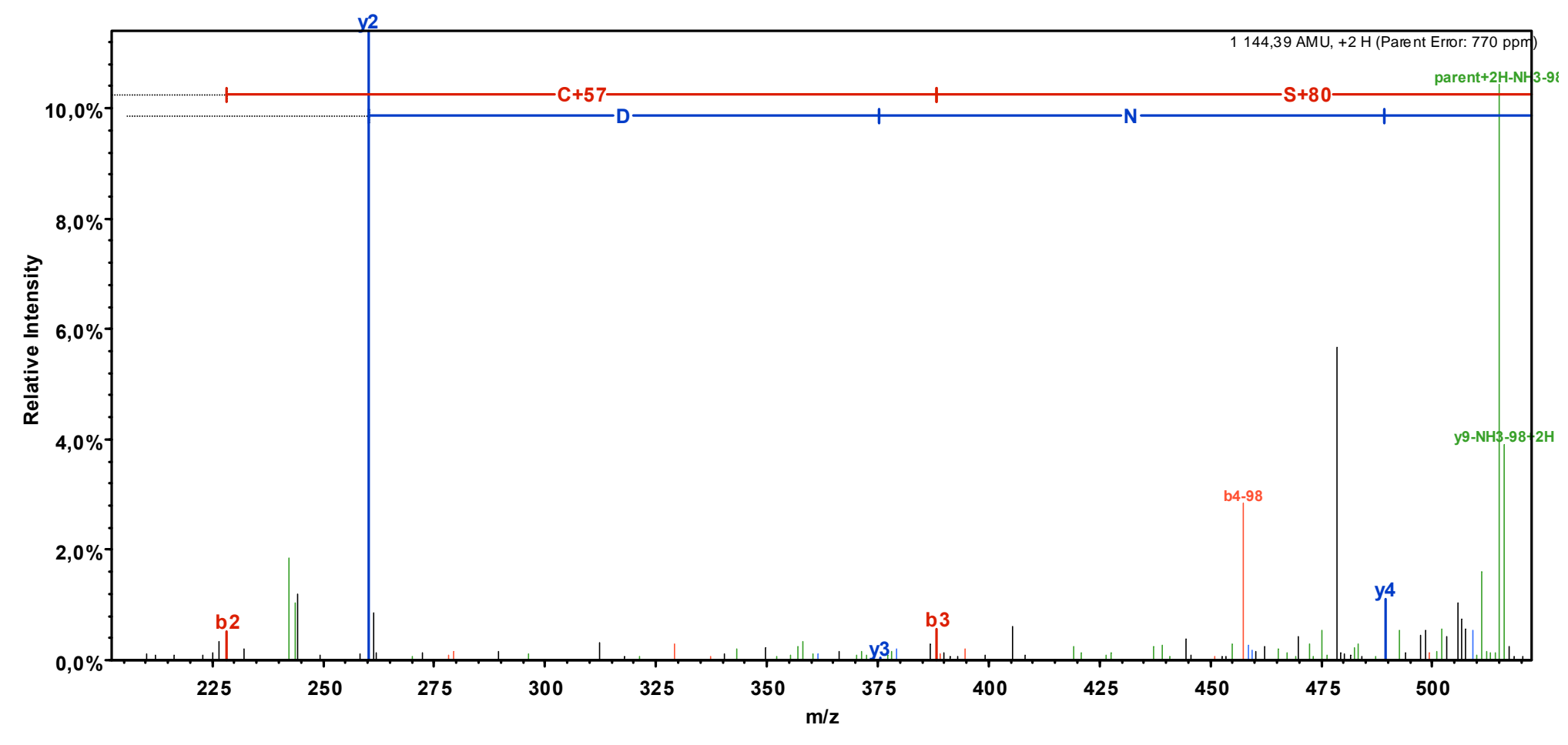

\section{$\underline{\text { Right of precursor }}$}

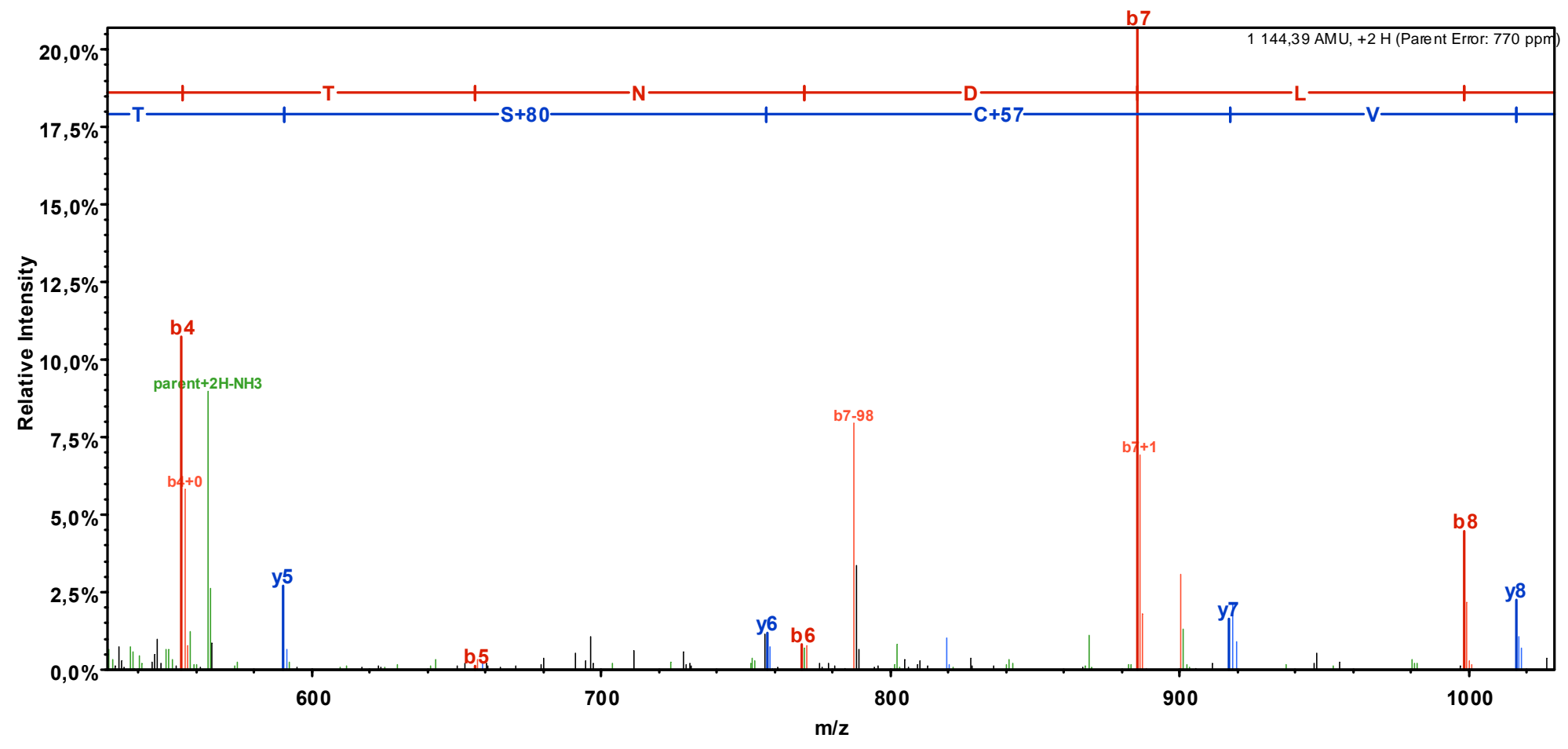




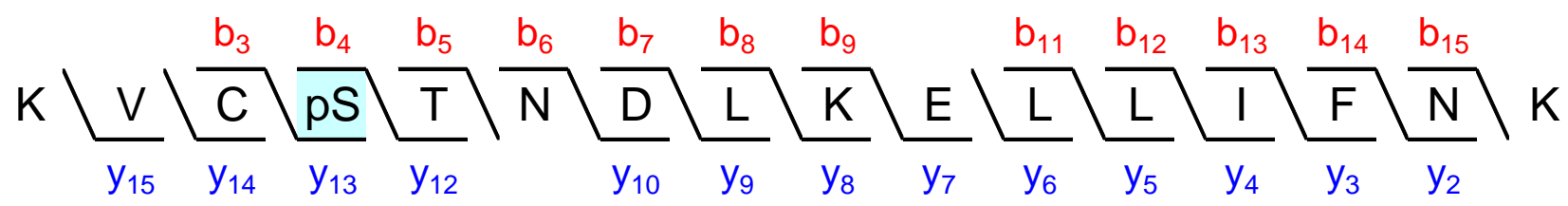

Sequest: $X$ Corr $=4.50, \mathrm{dCn} 1=0.07$ (between $1^{\text {st }}$ and $2^{\text {nd }}$ best matches), $\mathrm{dCn} 2=0.80$ (between $2^{\text {nd }}$ and $3^{\text {rd }}$ best matches).

Ascore $=13.27$

Sequest's second best match with XCorr $=4.17$ is to the same peptide sequence but with an alternative phosphorylation site: KVCSPTNDLKELLIFNK.

The weak Ascore indicates that, given the observed MS/MS spectrum, the phosphopeptide identified could either be Sequest's first or second best match (Nat Biotechnol. 2006;24(10):1285-92).

\section{MS/MS spectrum's fragmentation table}

\begin{tabular}{|c|c|c|c|c|c|c|c|c|c|c|}
\hline B & B Ions & $\mathrm{B}+2 \mathrm{H}$ & $\mathrm{B}-\mathrm{NH} 3$ & $\mathrm{~B}-\mathrm{H} 2 \mathrm{O}$ & AA & $Y$ Ions & $\mathrm{Y}+2 \mathrm{H}$ & $\mathrm{Y}-\mathrm{NH} 3$ & $\mathrm{Y}-\mathrm{H} 2 \mathrm{O}$ & $Y$ \\
\hline 1 & 129,1 & 65,1 & 112,1 & & $\mathrm{~K}$ & 2002,0 & 1001,5 & 1985,0 & 1984,0 & 16 \\
\hline 2 & 228,2 & 114,6 & 211,1 & & V & 1873,9 & 937.5 & 1856,9 & 1855,9 & 15 \\
\hline 3 & 388.2 & 194,6 & 371,2 & & $C+57$ & 1774,8 & 887.9 & 1757,8 & 1756,8 & 14 \\
\hline 4 & 555.2 & 278,1 & 538,2 & 537.2 & $S+80$ & 1614,8 & 807.9 & 1597,8 & 1596,8 & 13 \\
\hline 5 & 656.2 & 328,6 & 639.2 & 638.2 & $\mathbf{T}$ & 1447,8 & 724.4 & 1430,8 & 1429,8 & 12 \\
\hline 6 & 770.3 & 385,6 & 753.3 & 752,3 & $\mathrm{~N}$ & 1346,8 & 673.9 & 1329,7 & 1328,8 & 11 \\
\hline 7 & 885.3 & 443,2 & 868.3 & 867,3 & D & 1232,7 & 616.9 & 1215,7 & 1214,7 & 10 \\
\hline 8 & 998.4 & 499,7 & 981,4 & 980.4 & L & 1117,7 & 559.4 & 1100.7 & 1099,7 & 9 \\
\hline 9 & 1126.5 & 563,8 & 1109.5 & 1108,5 & K & 1004.6 & 502,8 & 987,6 & 986.6 & 8 \\
\hline 10 & 1255,5 & 628,3 & 1238.5 & 1237,5 & $E$ & 876.5 & 438,8 & 859.5 & 858,5 & 7 \\
\hline 11 & 1368,6 & 684.8 & 1351,6 & 1350,6 & L & 747.5 & 374.2 & 730.5 & & 6 \\
\hline 12 & 1481,7 & 741.4 & 1464,7 & 1463,7 & L & 634,4 & & 617.4 & & 5 \\
\hline 13 & 1594,8 & 797.9 & 1577,8 & 1576.8 & I & 521,3 & & 504,3 & & 4 \\
\hline 14 & 1741,9 & 871.4 & 1724,8 & 1723,8 & $F$ & 408.2 & & 391,2 & & 3 \\
\hline 15 & 1855,9 & 928.5 & 1838,9 & 1837,9 & $\mathrm{~N}$ & 261.2 & & 244.1 & & 2 \\
\hline 16 & 2002,0 & 1001,5 & 1985,0 & 1984,0 & K & 147,1 & & 130,1 & & 1 \\
\hline
\end{tabular}




\section{MS/MS spectrum}

\section{Full-range}

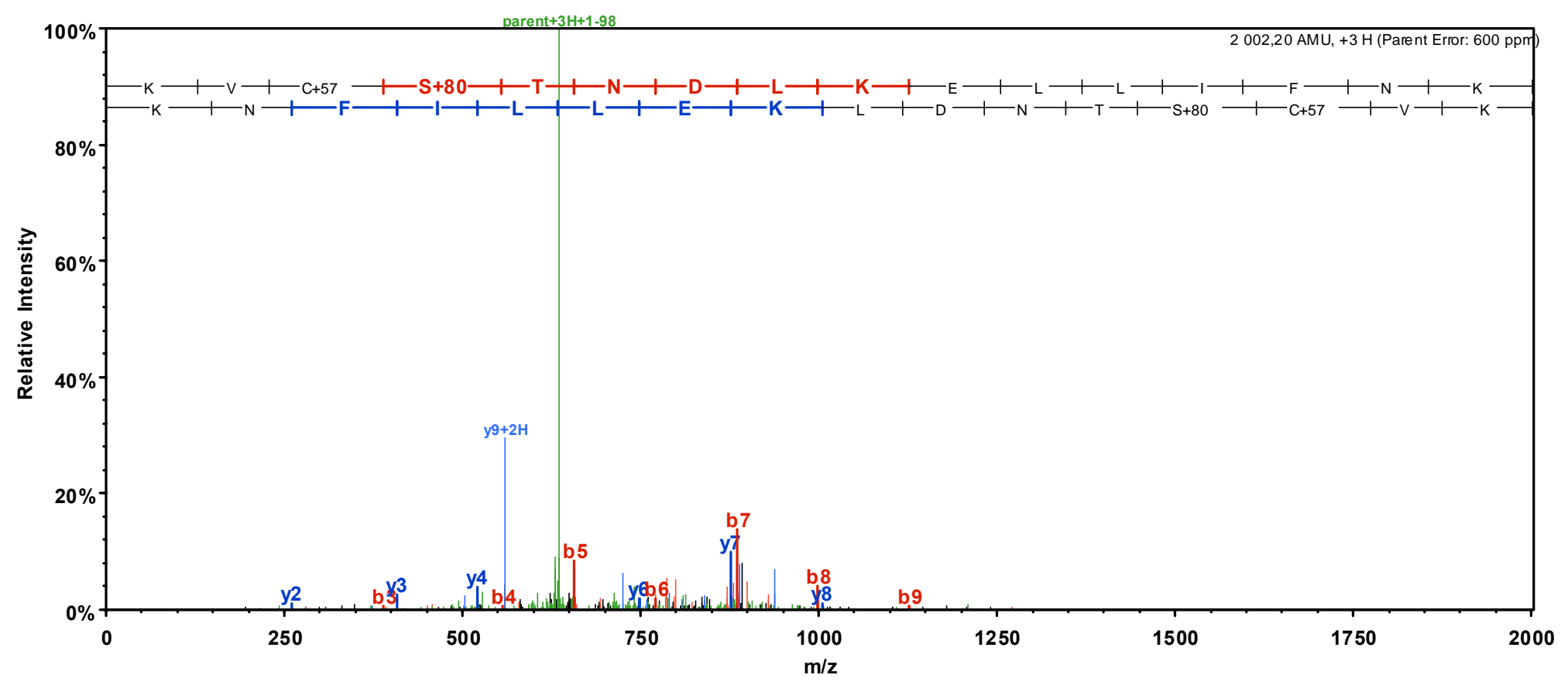

\section{$\underline{\text { Close to precursor }}$}

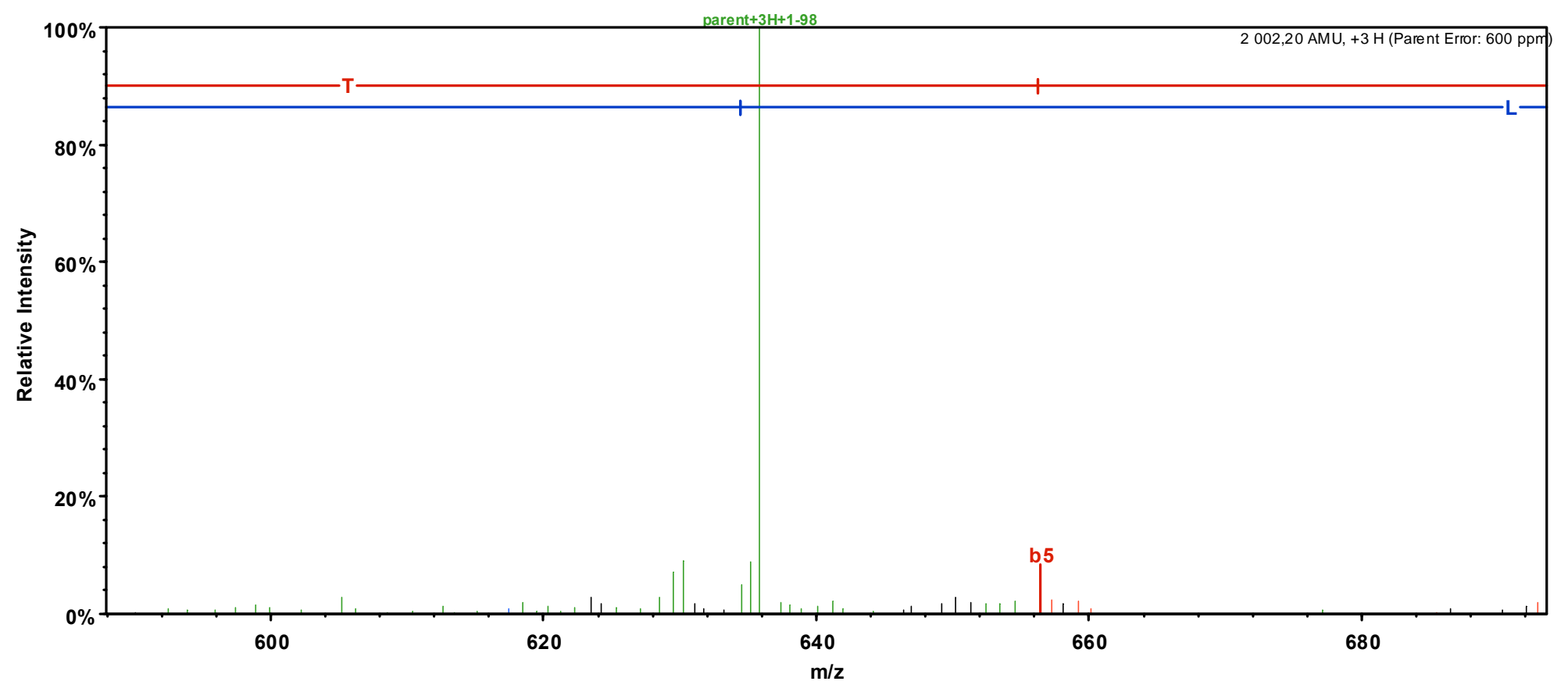




\section{Left of precursor}

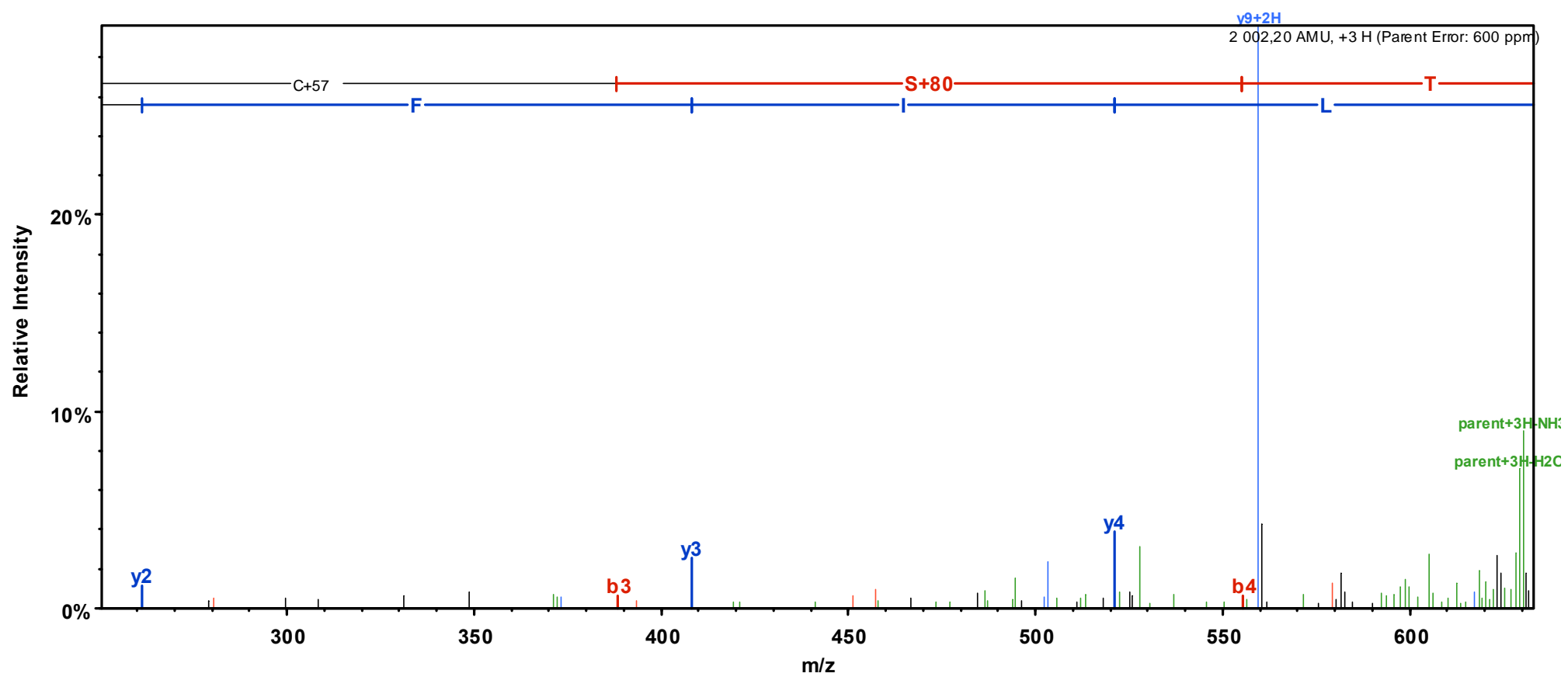

\section{$\underline{\text { Right of precursor }}$}

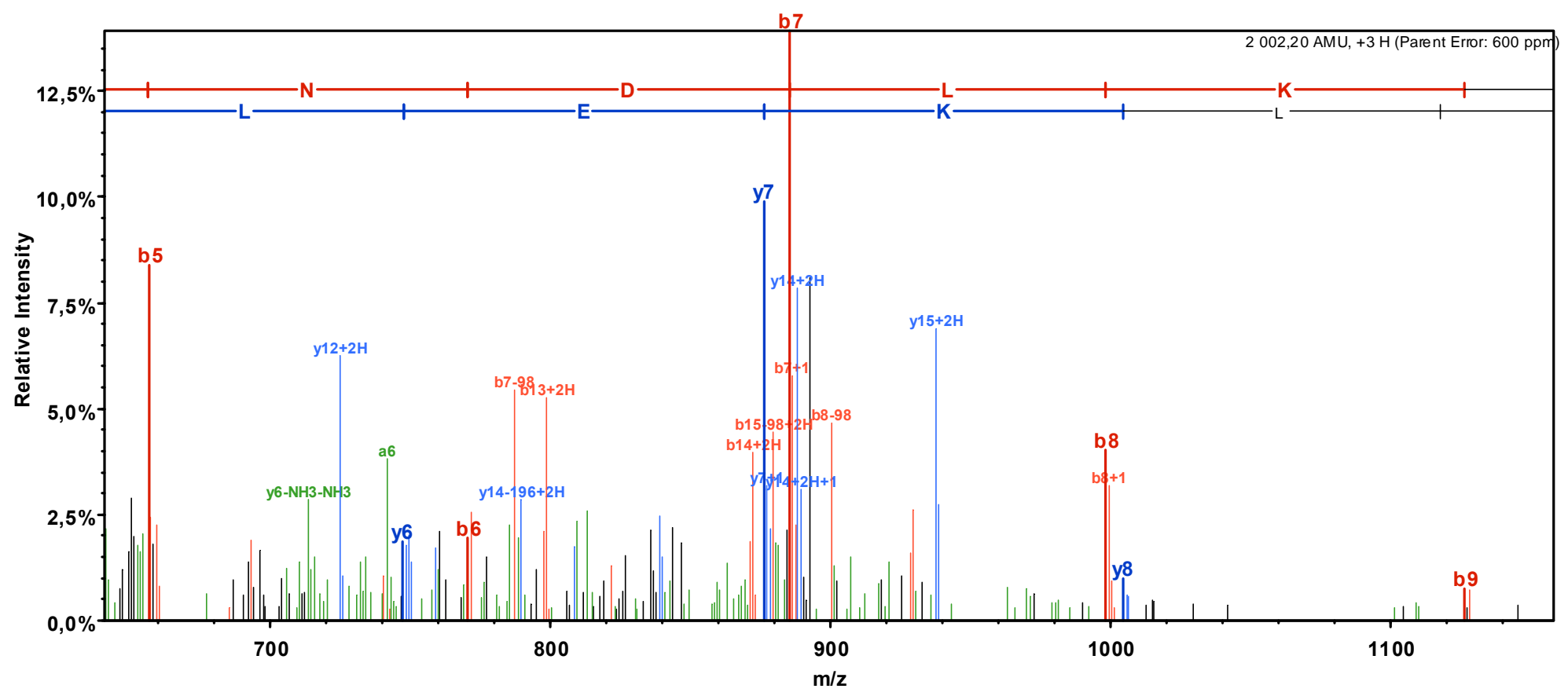




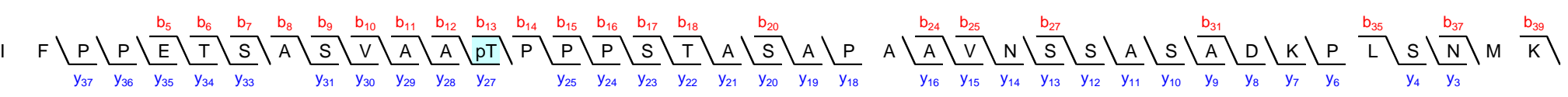

Sequest: $\mathrm{XCorr}=4.35, \mathrm{dCn} 1=0.003$ (between $1^{\text {st }}$ and $2^{\text {nd }}$ best matches), $\mathrm{dCn} 2=0.02$ (between $2^{\text {nd }}$ and $3^{\text {rd }}$ best matches), $\mathrm{dCn} 3=0.03$ (between $3^{\text {rd }}$ and $4^{\text {th }}$ best matches), $0.12 \mathrm{dCn} 4=$ (between $4^{\text {th }}$ and $5^{\text {th }}$ best matches).

Ascore $=8.92$.

Sequest's second, third and fourth best matches with XCorr $=4.34 .4 .27$ and 4.24 respectively are to the same peptide sequence but with alternative phosphorylation sites:
IFPPETSASVAATPPPPSTASAPAAVNSSASADKPLSNMK
IFPPETSASVAATPPPSPTASAPAAVNSSASADKPLSNMK
IFPPETSASVAATPPPSTAPSAPAAVNSSASADKPLSNMK

The weak Ascore indicates that, given the observed MS/MS spectrum, the phosphopeptide identified could be either of the above four (Nat Biotechnol. 2006;24(10):1285-92).

\section{MS/MS spectrum's fragmentation table}

\begin{tabular}{|c|c|c|c|c|c|c|c|c|c|c|}
\hline B & B Ions & $\mathrm{B}+2 \mathrm{H}$ & $\mathrm{B}-\mathrm{NH} 3$ & $\mathrm{~B}-\mathrm{H} 2 \mathrm{O}$ & AA & $Y$ Ions & $\mathrm{Y}+2 \mathrm{H}$ & $\mathrm{Y}-\mathrm{NH} 3$ & $\mathrm{Y}-\mathrm{H} 2 \mathrm{O}$ & $Y$ \\
\hline 1 & 114.1 & & & & I & 3863,8 & 1932,4 & 3846,8 & 3845,8 & 39 \\
\hline 2 & 261,2 & & & & $\mathbf{F}$ & 3750,8 & 1875.9 & 3733.7 & 3732.7 & 38 \\
\hline 3 & 358,2 & & & & $\mathbf{P}$ & 3603.7 & 1802,3 & 3586,7 & 3585,7 & 37 \\
\hline 4 & 455.3 & & & & $\mathbf{P}$ & 3506.6 & 1753.8 & 3489.6 & 3488,6 & 36 \\
\hline 5 & 584.3 & & & 566.3 & $E$ & 3409,6 & 1705.3 & 3392,6 & 3391,6 & 35 \\
\hline 6 & 685.4 & 343,2 & & 667,3 & $\mathbf{T}$ & 3280,5 & 1640.8 & 3263.5 & 3262,5 & 34 \\
\hline 7 & 772.4 & 386,7 & & 754,4 & S & 3179,5 & 1590.3 & 3162,5 & 3161,5 & 33 \\
\hline 8 & 843.4 & 422,2 & & 825,4 & A & 3092,5 & 1546,7 & 3075,4 & 3074,5 & 32 \\
\hline 9 & 930.5 & 465,7 & & 912,4 & S & 3021,4 & 1511.2 & 3004.4 & 3003.4 & 31 \\
\hline 10 & 1029.5 & 515,3 & & 1011,5 & V & 2934,4 & 1467.7 & 2917,4 & 2916.4 & 30 \\
\hline 11 & 1100.6 & 550,8 & & 1082.6 & A & 2835,3 & 1418.2 & 2818,3 & 2817,3 & 29 \\
\hline 12 & 1171,6 & 586,3 & & 1153.6 & A & 2764.3 & 1382.6 & 2747,3 & 2746.3 & 28 \\
\hline 13 & 1352.6 & 676,8 & & 1334.6 & $\mathbf{T}+\mathbf{8 0}$ & 2693.2 & 1347.1 & 2676,2 & 2675,2 & 27 \\
\hline 14 & \begin{tabular}{|l|l|}
1449.7 \\
\end{tabular} & 725,3 & & 1431,7 & P & 2512,2 & 1256,6 & 2495,2 & 2494,2 & 26 \\
\hline 15 & 1546.7 & 773,9 & & 1528.7 & $\mathbf{P}$ & 2415,2 & 1208.1 & 2398,2 & 2397,2 & 25 \\
\hline 16 & 1643,8 & 822.4 & & 1625,8 & $\mathbf{P}$ & 2318,1 & 1159.6 & 2301,1 & 2300,1 & 24 \\
\hline 17 & 1730.8 & 865.9 & & 1712,8 & S & 2221,1 & 1111.0 & 2204,1 & 2203,1 & 23 \\
\hline 18 & 1831,9 & 916.4 & & 1813,8 & $\mathbf{T}$ & 2134,0 & 1067.5 & 2117,0 & 2116,0 & 22 \\
\hline 19 & 1902,9 & 951,9 & & 1884,9 & A & 2033,0 & 1017.0 & 2016.0 & 2015,0 & 21 \\
\hline 20 & 1989,9 & 995.5 & & 1971,9 & $\mathrm{~s}$ & 1962.0 & 981.5 & 1944.9 & 1943,9 & 20 \\
\hline 21 & 2061,0 & 1031,0 & & 2042,9 & A & 1874,9 & 938.0 & 1857,9 & 1856,9 & 19 \\
\hline 22 & 2158,0 & 1079,5 & & 2140,0 & $\mathbf{P}$ & 1803.9 & 902,4 & 1786.9 & 1785,9 & 18 \\
\hline 23 & 2229,0 & 1115,0 & & 2211,0 & A & 1706.8 & 853,9 & 1689,8 & 1688.8 & 17 \\
\hline 24 & 2300,1 & 1150.5 & & 2282,1 & A & 1635,8 & 818.4 & 1618,8 & 1617,8 & 16 \\
\hline 25 & 2399,2 & 1200.1 & & 2381,1 & V & 1564.8 & 782.9 & 1547,7 & 1546,8 & 15 \\
\hline 26 & 2513,2 & 1257,1 & 2496,2 & 2495,2 & $\mathrm{~N}$ & 1465.7 & 733.4 & 1448,7 & 1447,7 & 14 \\
\hline 27 & 2600,2 & 1300.6 & 2583,2 & 2582,2 & S & 1351.7 & 676,3 & 1334.6 & 1333.6 & 13 \\
\hline 28 & 2687,3 & 1344.1 & 2670,2 & 2669,2 & S & 1264.6 & 632,8 & 1247.6 & 1246,6 & 12 \\
\hline 29 & 2758,3 & 1379,7 & 2741,3 & 2740,3 & A & 1177.6 & 589,3 & 1160,6 & 1159,6 & 11 \\
\hline 30 & 2845,3 & 1423,2 & 2828,3 & 2827,3 & S & 1106.6 & 553,8 & 1089,5 & 1088.5 & 10 \\
\hline 31 & 2916.4 & 1458.7 & 2899,3 & 2898,4 & A & 1019.5 & 510,3 & 1002.5 & 1001.5 & 9 \\
\hline 32 & 3031,4 & 1516,2 & 3014,4 & 3013.4 & D & 948.5 & 474,7 & 931,5 & 930,5 & 8 \\
\hline 33 & 3159,5 & 1580,2 & 3142,5 & 3141,5 & K & 833.5 & 417,2 & 816.4 & 815,4 & 7 \\
\hline 34 & 3256.5 & 1628,8 & 3239.5 & 3238.5 & $\mathbf{P}$ & 705.4 & 353,2 & 688,3 & 687.4 & 6 \\
\hline 35 & 3369,6 & 1685.3 & 3352,6 & 3351,6 & L & 608,3 & & 591,3 & 590,3 & 5 \\
\hline 36 & 3456,7 & 1728.8 & 3439,6 & 3438.6 & S & 495.2 & & 478,2 & 477,2 & 4 \\
\hline 37 & 3570,7 & 1785.9 & 3553,7 & 3552,7 & $\mathrm{~N}$ & 408.2 & & 391,2 & & 3 \\
\hline 38 & 3717,7 & 1859,4 & 3700,7 & 3699,7 & $M+16$ & 294,1 & & 277,1 & & 2 \\
\hline 39 & 3863,8 & 1932.4 & 3846,8 & 3845,8 & $\mathbf{K}$ & 147,1 & & 130,1 & & 1 \\
\hline
\end{tabular}




\section{MS/MS spectrum}

Note: Base peak (m/z 1202.6) is y37+3H (PPETSASVAATPPPpSTASAPAAVNSSASADKPLSNMK, theoretical m/z of 1201.9) and is not unexpected since fragments with $\mathrm{N}$-terminal proline are typically very intense. It was not identified by the visualisation software (Scaffold) because it does not handle triply-charged fragments. Simarly, the unidentified fragment at $\mathrm{m} / \mathrm{z} 1170$ is $y 36+3 \mathrm{H}$, another fragment with an $\mathrm{N}$-terminal proline.

\section{Full-range}

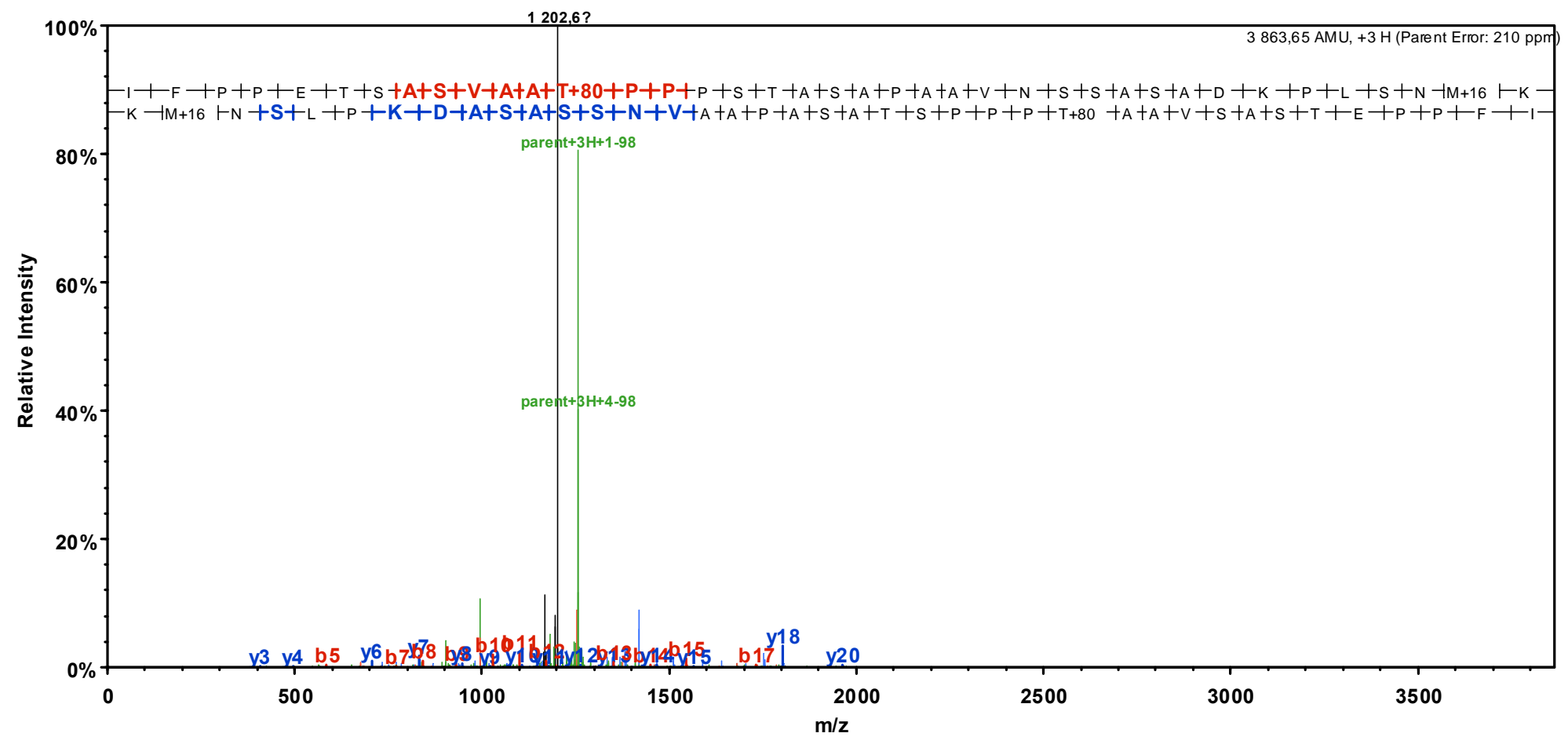

\section{Close to precursor}

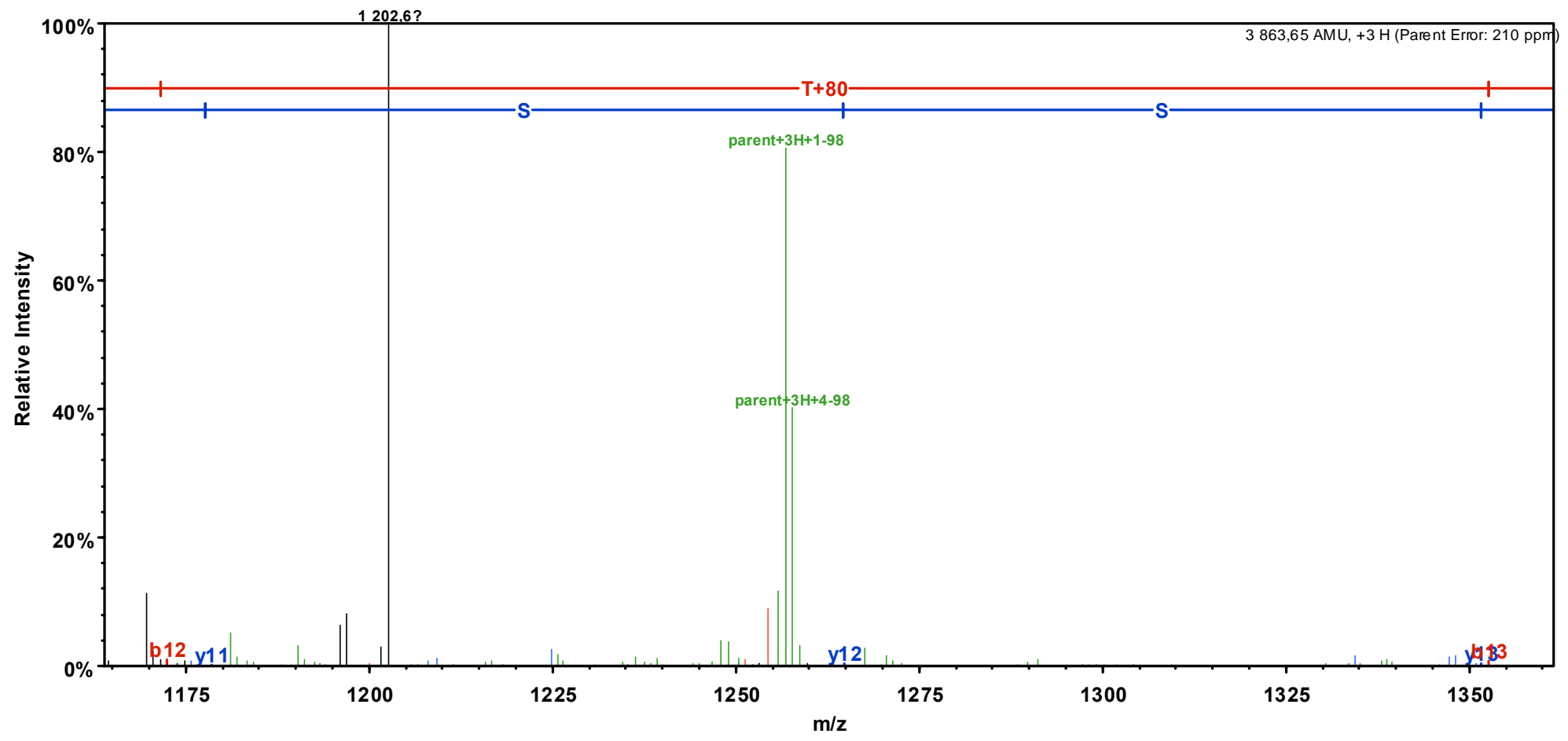




\section{Left of precursor}

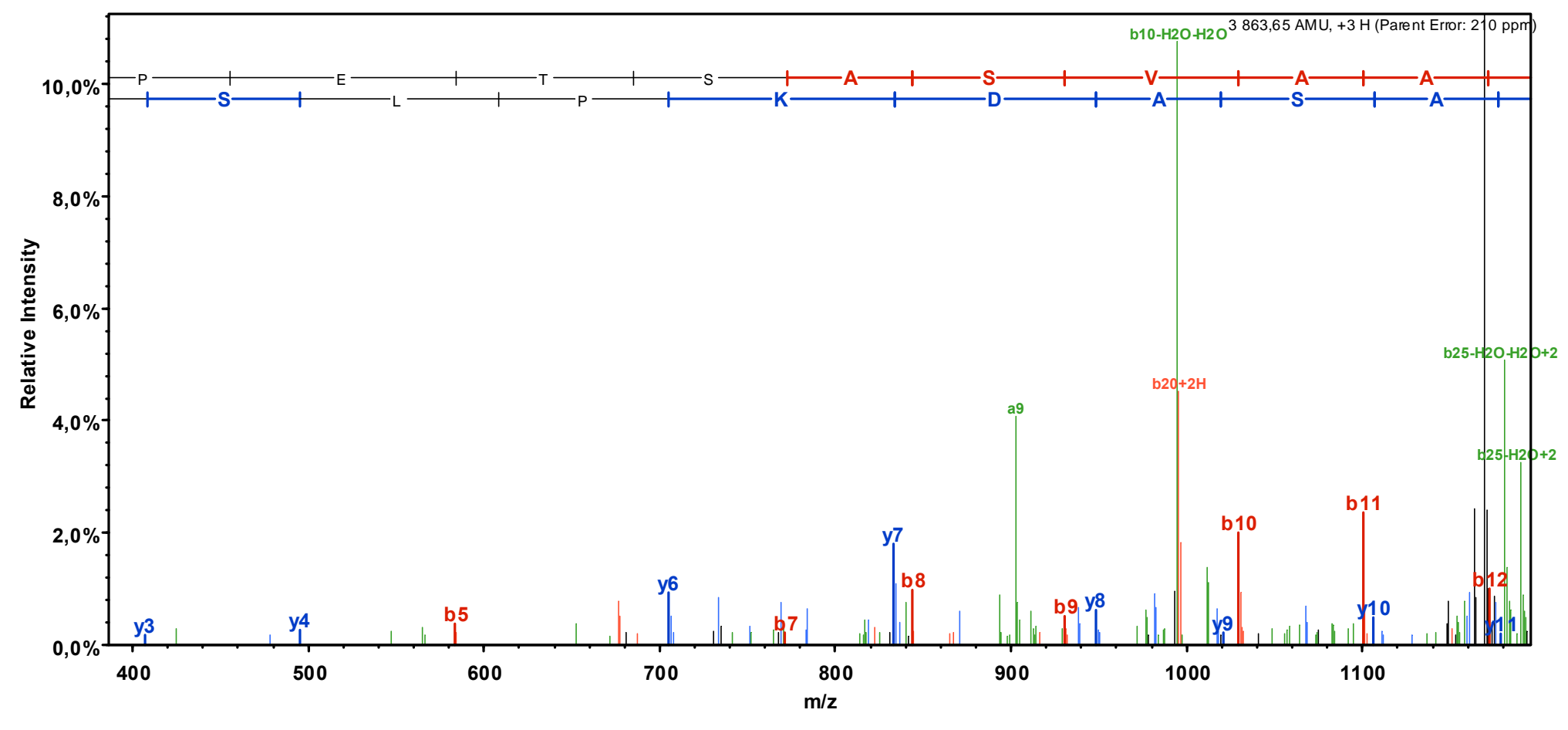

\section{$\underline{\text { Right of precursor }}$}

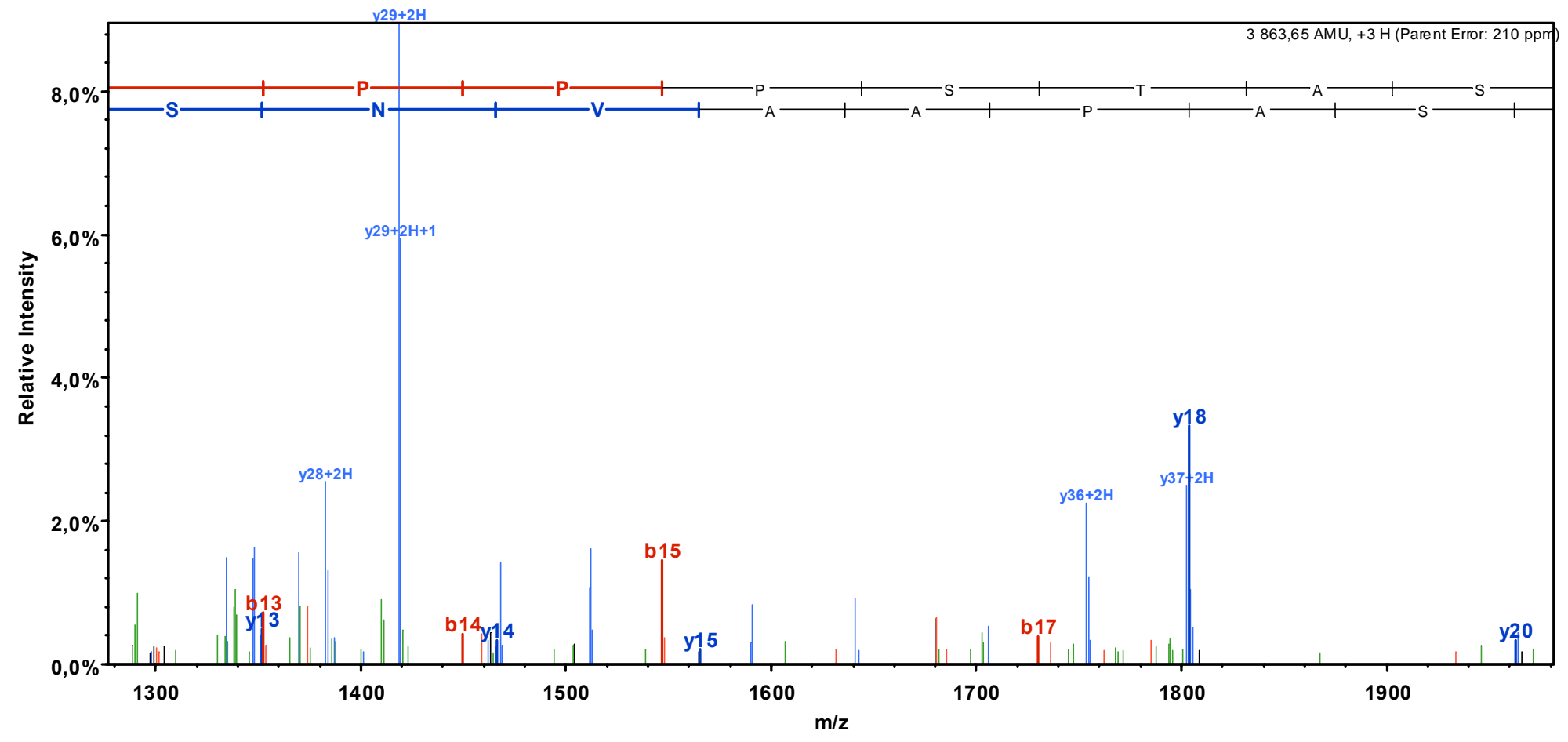




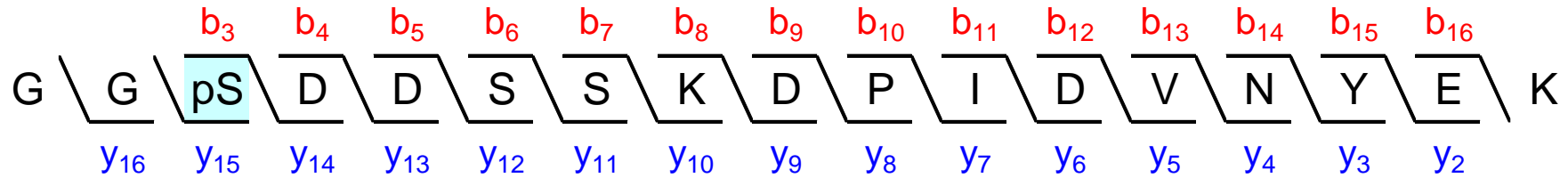

Sequest: XCorr $=4.85, \mathrm{dCn}=0.11$.

Ascore $=53.29$

Phosphorylation site is localized with $\geq 99 \%$ certainty as Ascore $\geq 20$ (Nat Biotechnol. 2006;24(10):1285-92).

\section{MS/MS spectrum's fragmentation table}

\begin{tabular}{|c|c|c|c|c|c|c|c|c|c|c|}
\hline B & B Ions & $\mathrm{B}+2 \mathrm{H}$ & $\mathrm{B}-\mathrm{NH} 3$ & $\mathrm{~B}-\mathrm{H} 2 \mathrm{O}$ & AA & Y Ions & $\mathrm{Y}+2 \mathrm{H}$ & $\mathrm{Y}-\mathrm{NH} 3$ & $\mathrm{Y}-\mathrm{H} 2 \mathrm{O}$ & $Y$ \\
\hline 1 & 58,0 & & & & $\mathbf{G}$ & 1905,8 & 953.4 & 1888.7 & 1887,8 & 17 \\
\hline 2 & 115,1 & & & & G & 1848,8 & 924.9 & 1831,7 & 1830,7 & 16 \\
\hline 3 & 282.0 & & & 264,0 & $5+80$ & 1791,7 & 896.4 & 1774,7 & 1773,7 & 15 \\
\hline 4 & 397.1 & & & 379,1 & D & 1624,7 & 812.9 & 1607,7 & 1606,7 & 14 \\
\hline 5 & 512.1 & & & 494.1 & D & 1509,7 & 755.4 & 1492,7 & 1491,7 & 13 \\
\hline 6 & 599.1 & 300.1 & & 581.1 & S & 1394,7 & 697.8 & 1377.7 & 1376.7 & 12 \\
\hline 7 & 686.2 & 343,6 & & 668.2 & S & 1307,6 & 654.3 & 1290,6 & 1289,6 & 11 \\
\hline 8 & 814.3 & 407.6 & 797.2 & 796.3 & K & 1220,6 & 610.8 & 1203,6 & 1202,6 & 10 \\
\hline 9 & 929.3 & 465.1 & 912,3 & 911,3 & D & 1092.5 & 546.8 & 1075,5 & 1074,5 & 9 \\
\hline 10 & 1026.3 & 513,7 & 1009.3 & 1008.3 & $\mathbf{P}$ & 977.5 & 489.3 & 960.5 & 959.5 & 8 \\
\hline 11 & 1139.4 & 570,2 & 1122,4 & 1121.4 & I & 880.4 & 440.7 & 863.4 & 862,4 & 7 \\
\hline 12 & 1254.5 & 627.7 & 1237.4 & 1236.4 & D & 767.4 & 384.2 & 750.3 & 749.3 & 6 \\
\hline 13 & 1353,5 & 677.3 & 1336.5 & 1335.5 & V & 652.3 & & 635,3 & 634,3 & 5 \\
\hline 14 & 1467,6 & 734.3 & 1450.5 & 1449.6 & $\mathrm{~N}$ & 553.3 & & 536.2 & 535.3 & 4 \\
\hline 15 & 1630,6 & 815.8 & 1613,6 & 1612,6 & $\mathbf{Y}$ & 439.2 & & 422,2 & 421,2 & 3 \\
\hline 16 & 1759,7 & 880.3 & 1742,6 & 1741,7 & E & 276.2 & & 259,1 & 258,1 & 2 \\
\hline 17 & 1905,8 & 953.4 & 1888,7 & 1887,8 & K & 147,1 & & 130,1 & & 1 \\
\hline
\end{tabular}




\section{MS/MS spectrum}

\section{Full-range}

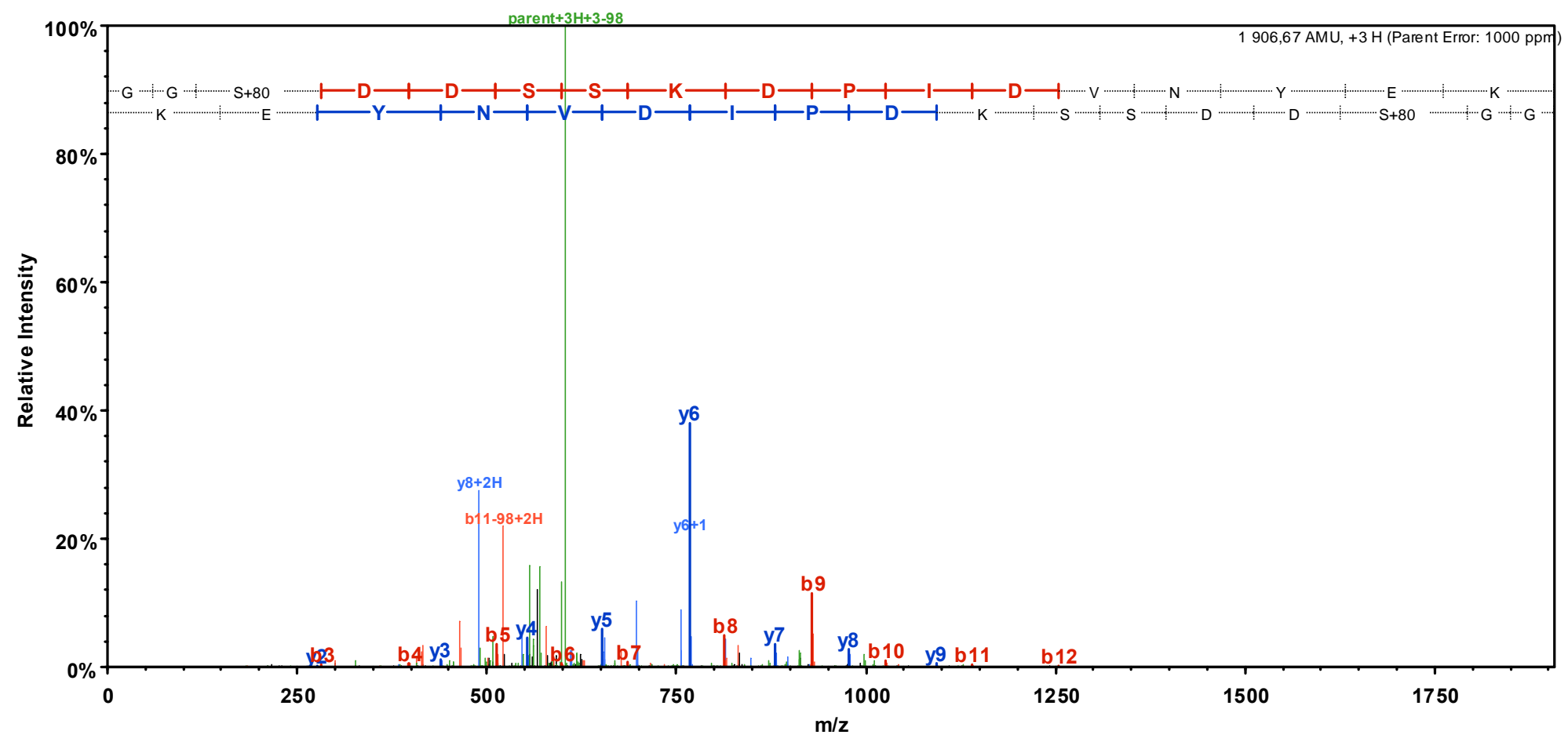

\section{$\underline{\text { Close to precursor }}$}

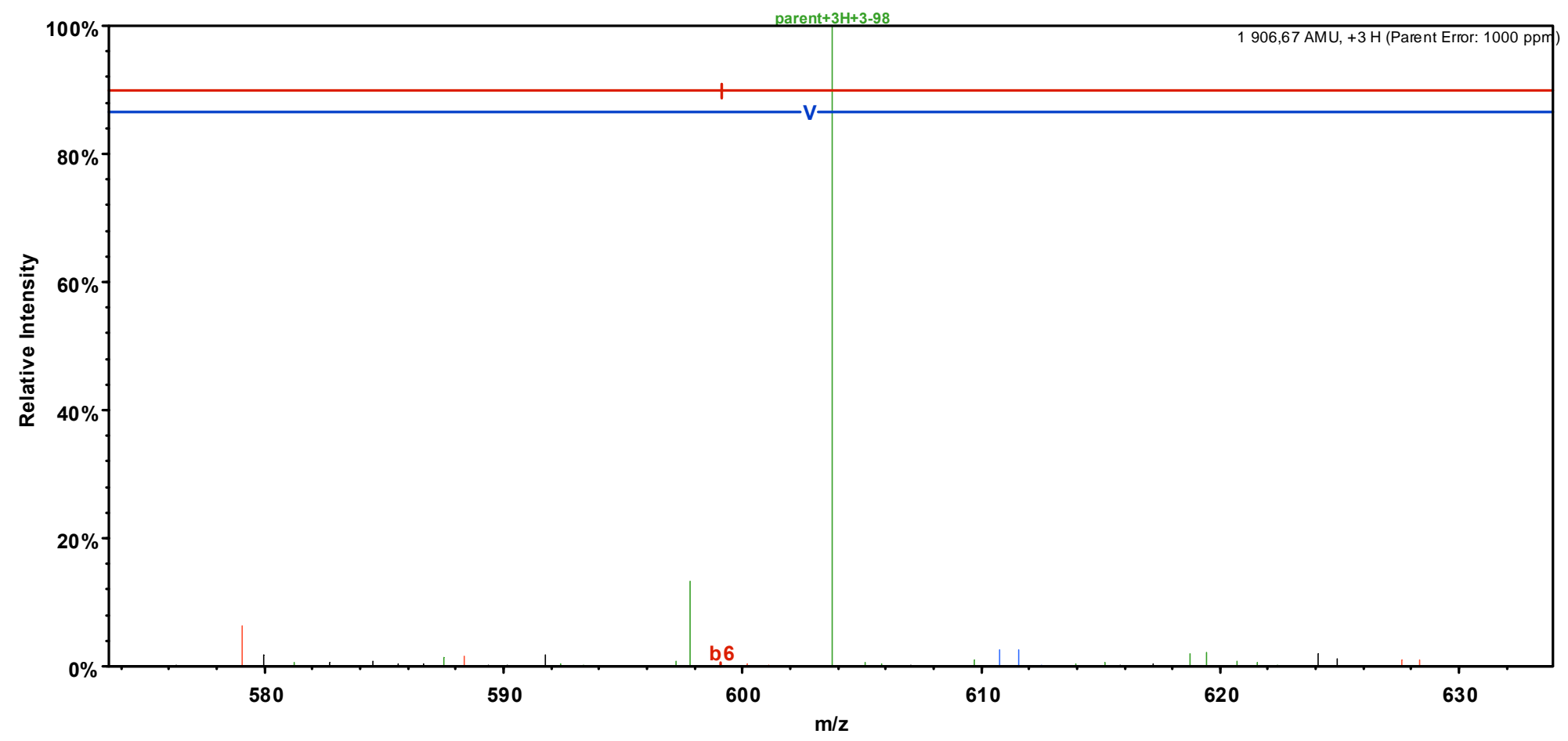




\section{Left of precursor}

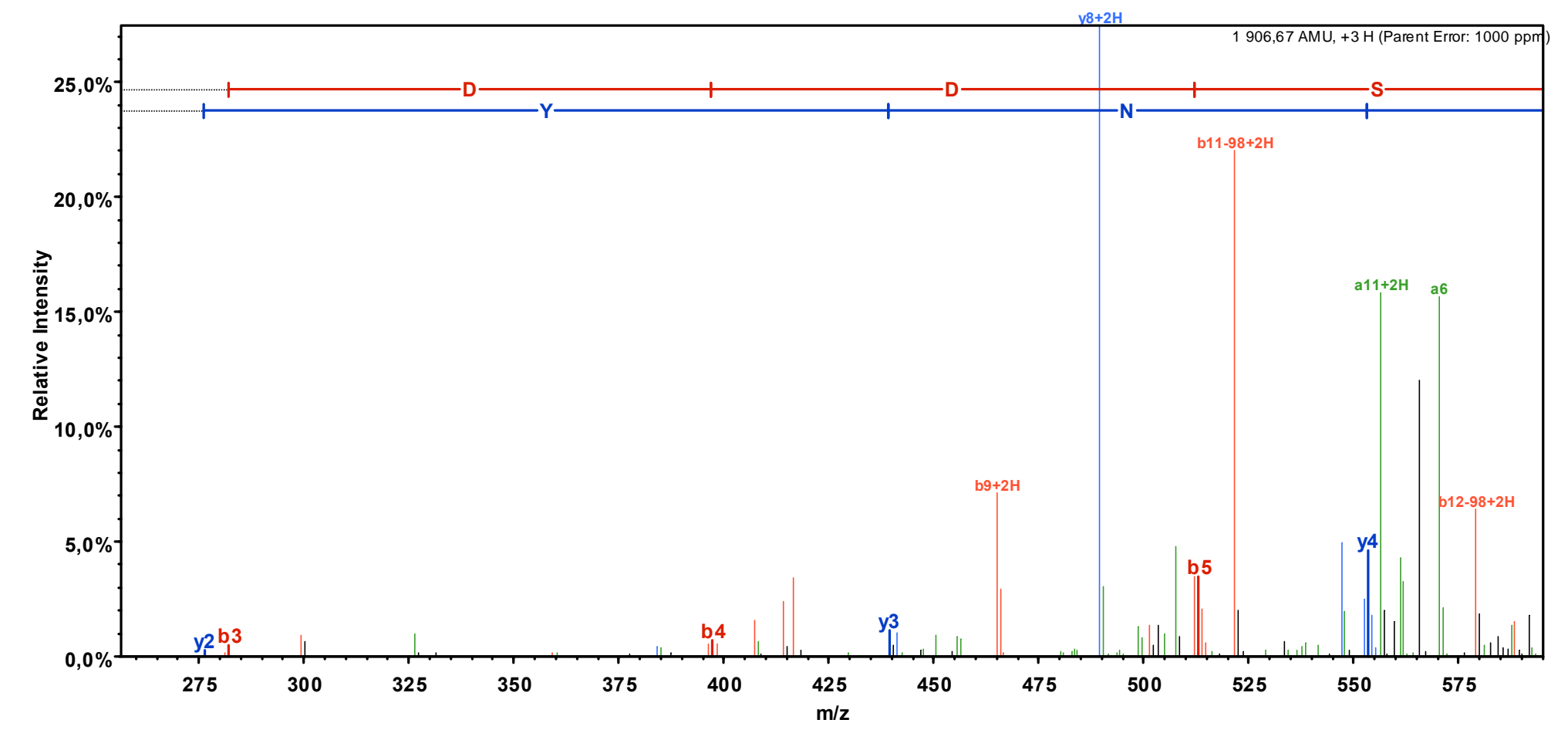

\section{$\underline{\text { Right of precursor }}$}

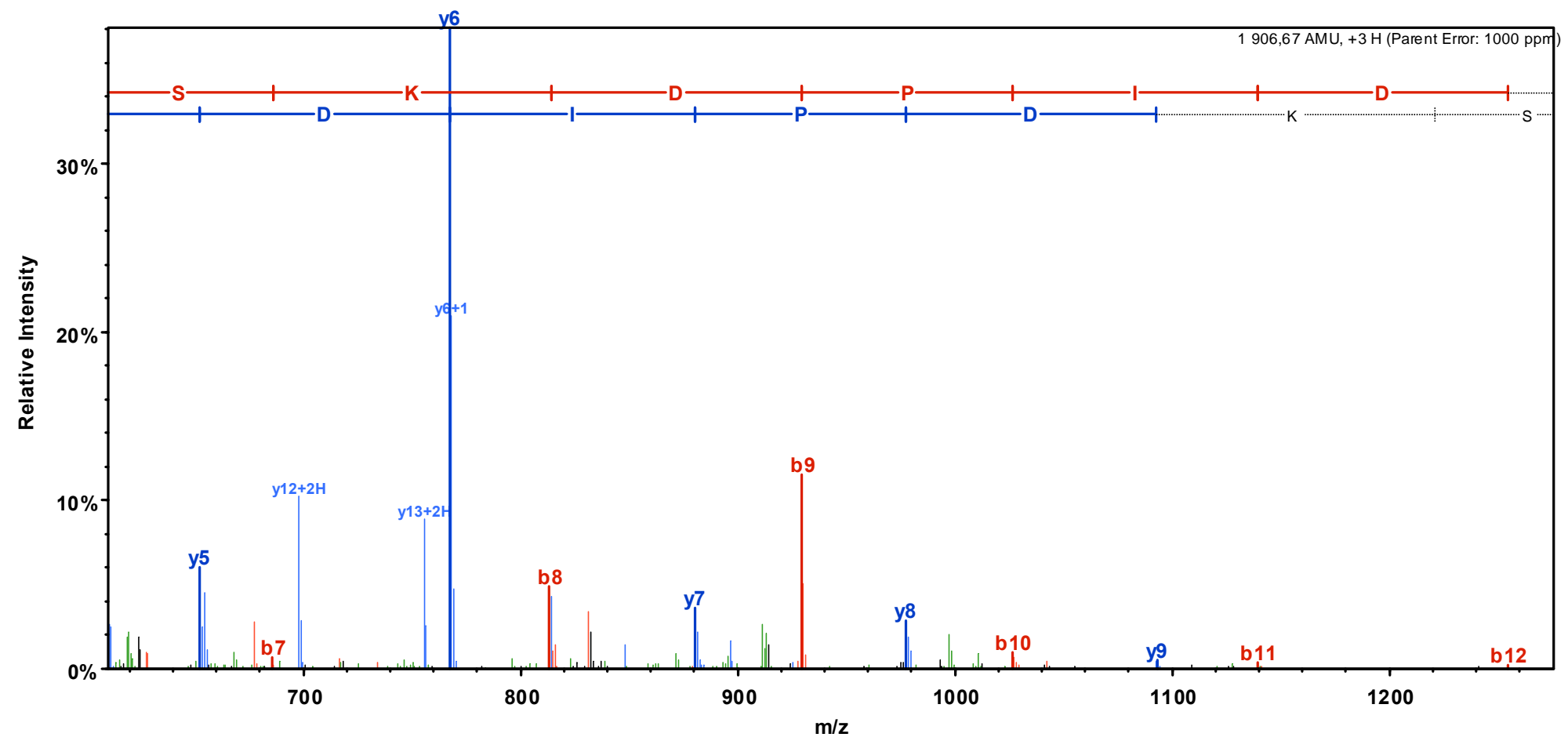


MALDI-TOF MS spectrum

Full range

+TOF MS: 60 MCA scans from PARP1_tio2_20061206_02.wiff
a=3.56095657219908010e-004, to =-8.76782082264835480e+00

Max. 793.0 counts.

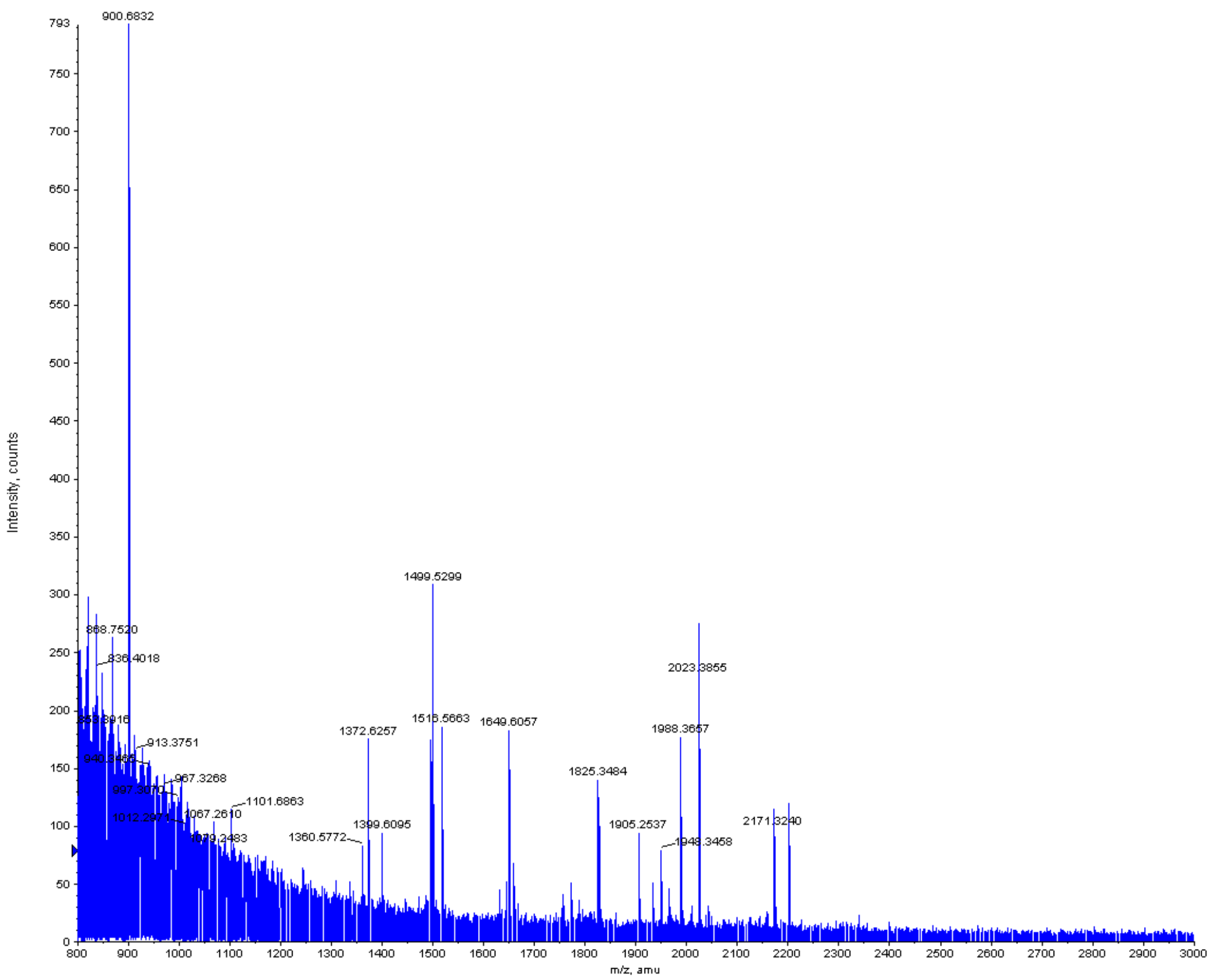




\section{First partial (zoomed) spectrum showing resolved isotopic envelopes}

\section{KEWVpTPKEFR}

Theoretical $\mathrm{MH}+$ (monoisotopic): $\mathrm{m} / \mathrm{z} 1399.68$

Observed $\mathrm{MH}+$ (monoisotopic): $\mathrm{m} / \mathrm{z} 1399.61$

$\delta m=-0.09 \mathrm{Th}$

\section{KCSESIPKDpSLR}

Theoretical $\mathrm{MH}+$ (monoisotopic): m/z 1499.69

Observed $\mathrm{MH}+$ (monoisotopic): m/z 1499.53

$\delta m=-0.16 \mathrm{Th}$

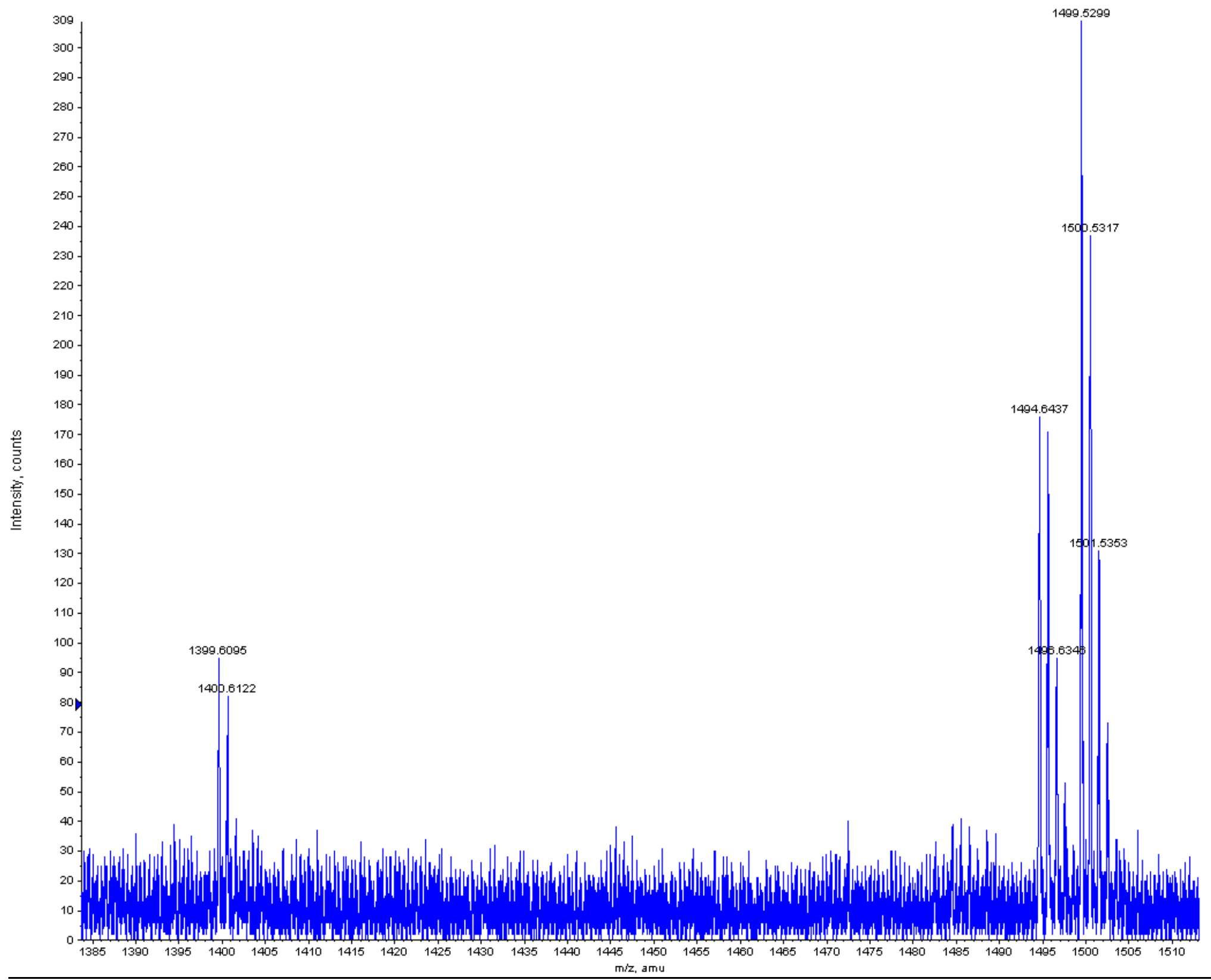




\section{GGpSDDSSKDPIDVNYEK}

Theoretical $\mathrm{MH}+$ (monoisotopic): $\mathrm{m} / \mathrm{z} 1905.78$

Observed $\mathrm{MH}+$ (monoisotopic): $\mathrm{m} / \mathrm{z} 1905.25$

$\delta m=-0.53 T h$

+TOF MS: 60 MCA scans from PARP1_ti02_20061206_02. mitf

$a=3.56095657219908010 \mathrm{e}-004, t 0=-8.76782082264835480 \mathrm{e}+001$

Max. 793.0 counts.

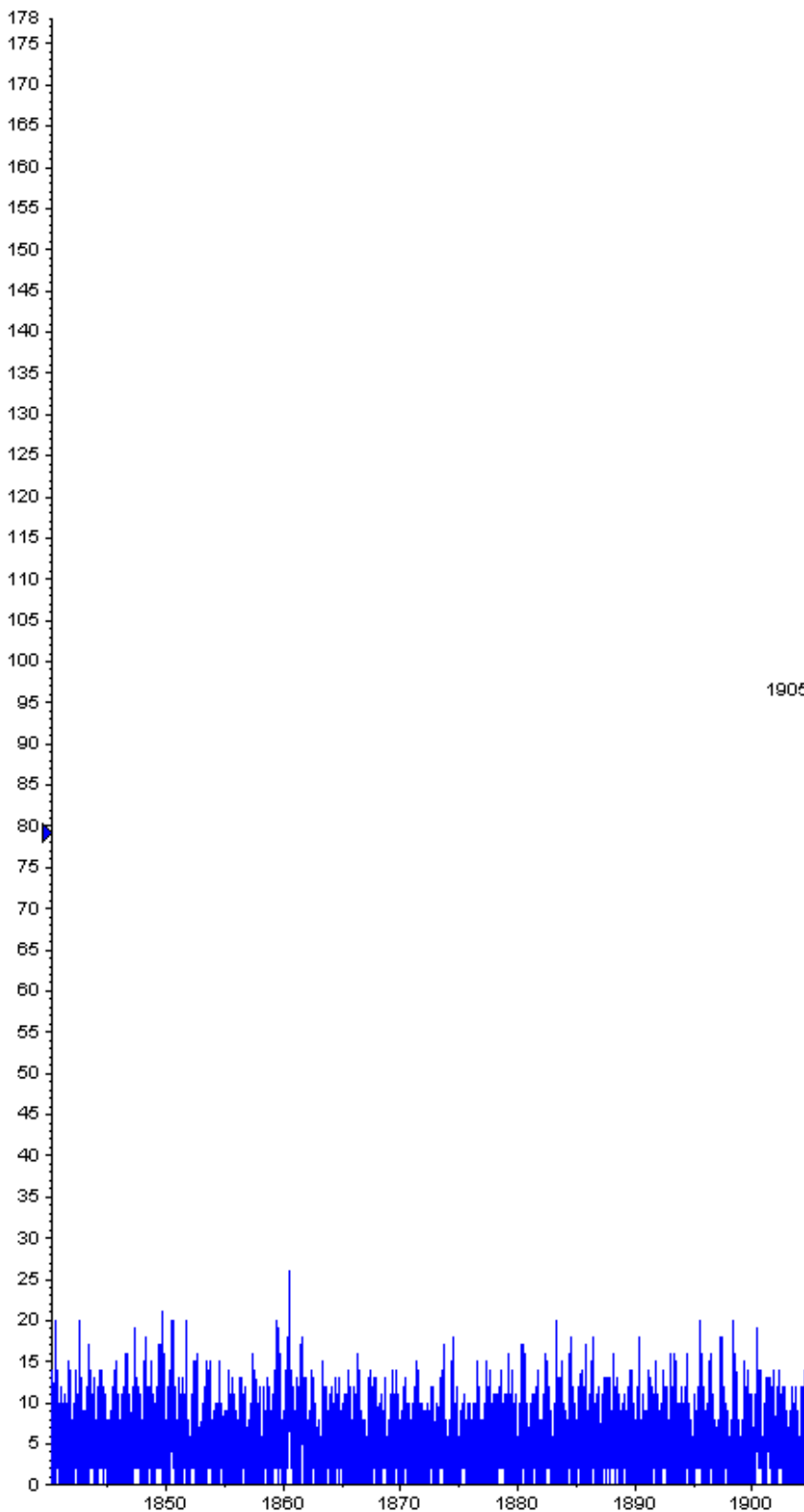

1905.2537
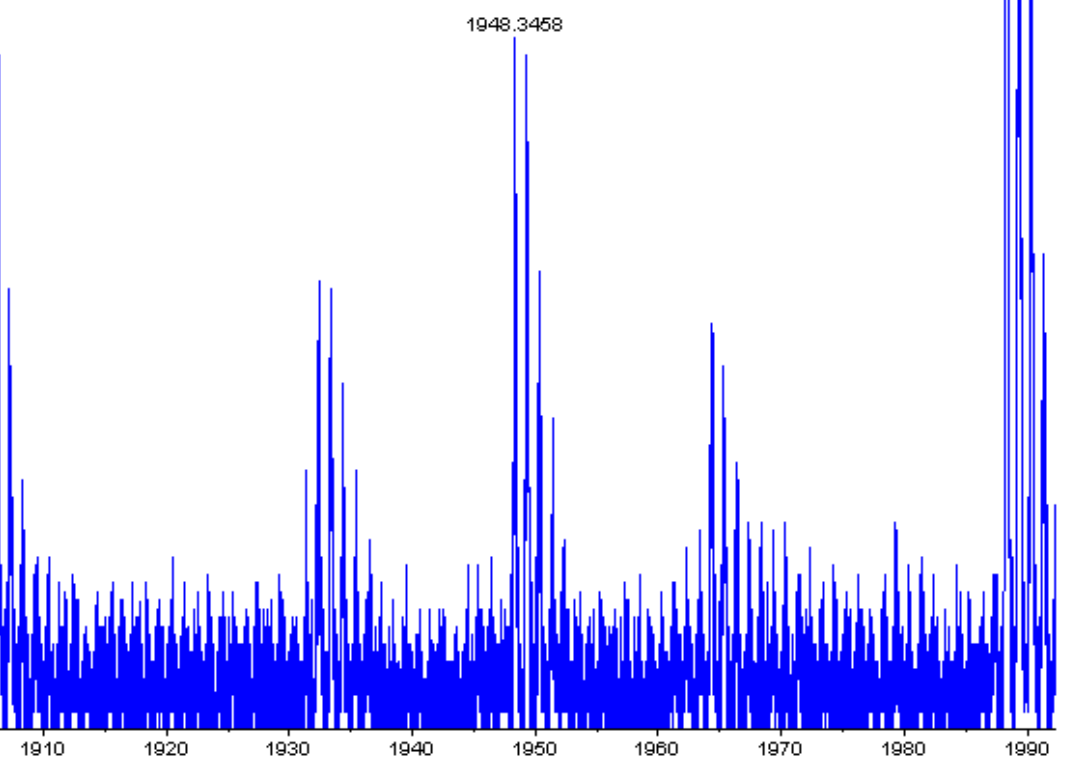

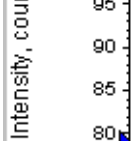


Supplementary mass spectrometric data:

PARP-1, phosphorylated by extracellular signal-regulated kinase-1 (ERK-1)

\section{Spectrum 7674.3412.3412.2.dta (charge state: +2)}

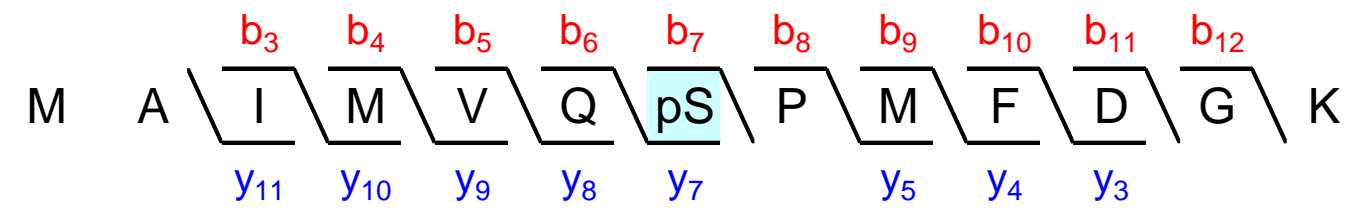

Sequest: $\mathrm{XCorr}=4.21, \mathrm{dCn}=0.46$.

Ascore $=$ Not available (only one possible phosphorylation site).

The phosphorylation is located at the only possible phosphorylation site (no other S, T, Y within the peptide).

\section{MS/MS spectrum's fragmentation table}

\begin{tabular}{|c|c|c|c|c|c|c|c|c|c|c|}
\hline B & B Ions & $\mathrm{B}+2 \mathrm{H}$ & $\mathrm{B}-\mathrm{NH} 3$ & $\mathrm{~B}-\mathrm{H} 2 \mathrm{O}$ & AA & $Y$ Ions & $\mathrm{Y}+2 \mathrm{H}$ & $\mathrm{Y}-\mathrm{NH} 3$ & $\mathrm{Y}-\mathrm{H} 2 \mathrm{O}$ & $Y$ \\
\hline 1 & 148,0 & & & & $M+16$ & 1566.6 & 783,8 & 1549,6 & 1548,6 & 13 \\
\hline 2 & 219,1 & & & & A & 1419.6 & 710,3 & 1402,6 & 1401,6 & 12 \\
\hline 3 & 332.2 & & & & I & 1348.6 & 674.8 & 1331.5 & 1330,6 & 11 \\
\hline 4 & 479.2 & & & & $M+16$ & 1235.5 & 618.2 & 1218.5 & 1217.5 & 10 \\
\hline 5 & 578.3 & & & & $\mathrm{~V}$ & 1088.4 & 544,7 & 1071.4 & 1070.4 & 9 \\
\hline 6 & 706.3 & 353,7 & 689,3 & & $\mathbf{Q}$ & 989.4 & 495.2 & 972,4 & 971,4 & 8 \\
\hline 7 & 873.3 & 437,2 & 856.3 & 855,3 & $S+80$ & 861.3 & 431,2 & 844,3 & 843.3 & 7 \\
\hline 8 & 970.4 & 485.7 & 953.4 & 952,4 & $\mathbf{P}$ & 694,3 & 347,7 & 677,3 & 676,3 & 6 \\
\hline 9 & 1101.4 & 551,2 & 1084.4 & 1083.4 & M & 597.3 & & 580,2 & 579,3 & 5 \\
\hline 10 & 1248.5 & 624.7 & 1231.5 & 1230.5 & $\mathbf{F}$ & 466.2 & & 449,2 & 448,2 & 4 \\
\hline 11 & 1363.5 & 682.3 & 1346.5 & 1345.5 & D & 319.2 & & 302,1 & 301,2 & 3 \\
\hline 12 & 1420.5 & 710,8 & 1403.5 & 1402,5 & G & 204,1 & & 187,1 & & 2 \\
\hline 13 & 1566,6 & 783,8 & 1549,6 & 1548,6 & K & 147,1 & & 130,1 & & 1 \\
\hline
\end{tabular}




\section{MS/MS spectrum}

\section{Full-range}

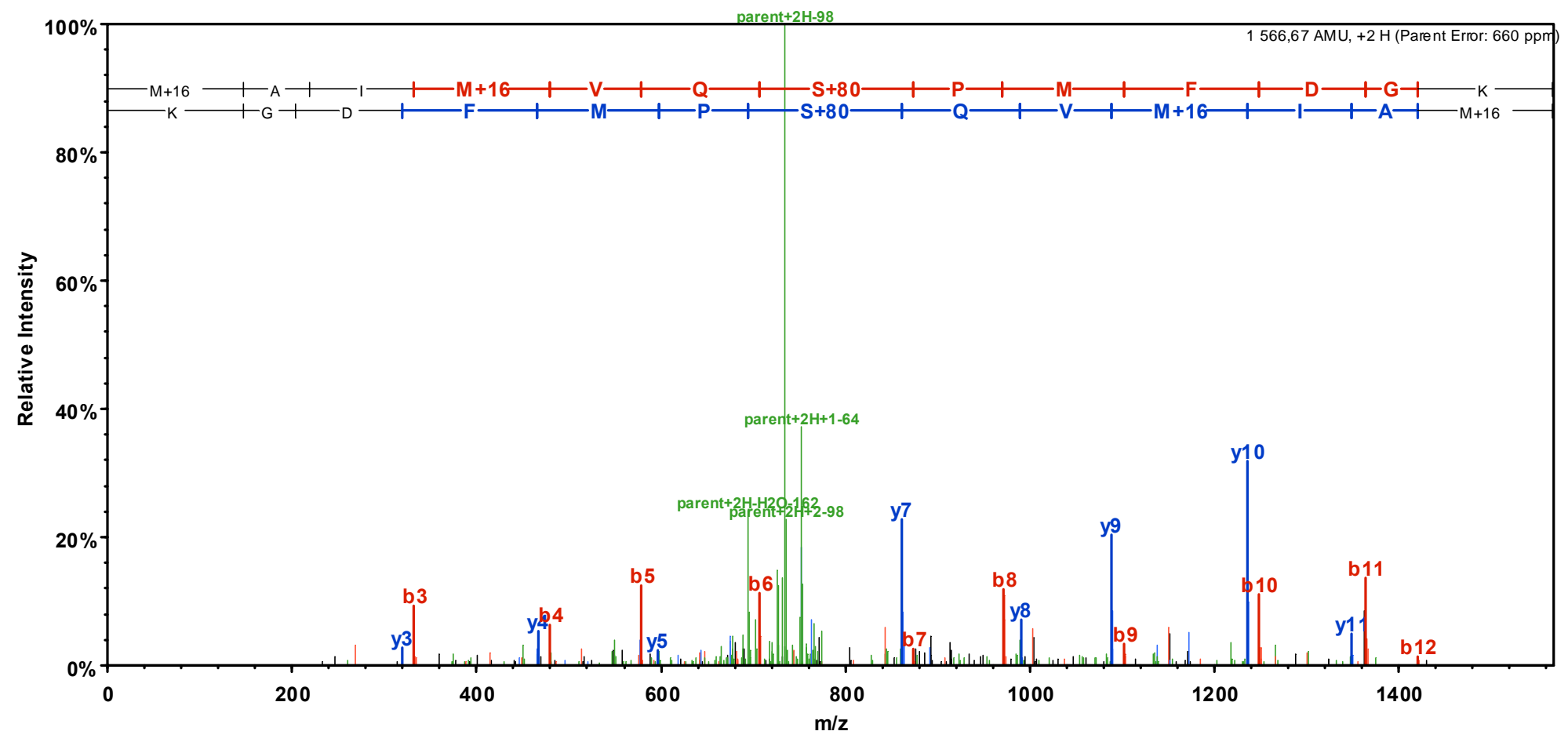

\section{$\underline{\text { Close to precursor }}$}

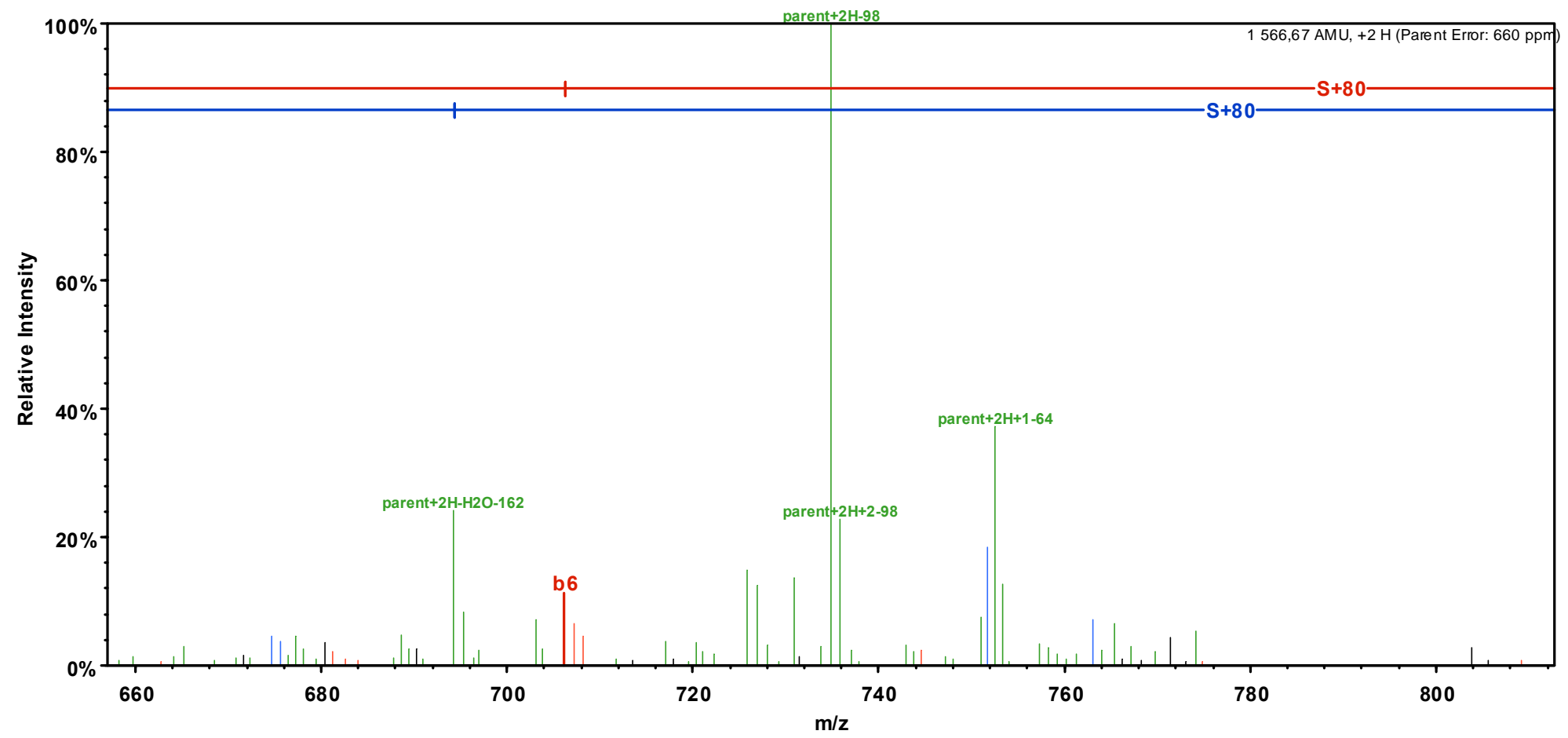




\section{Left of precursor}

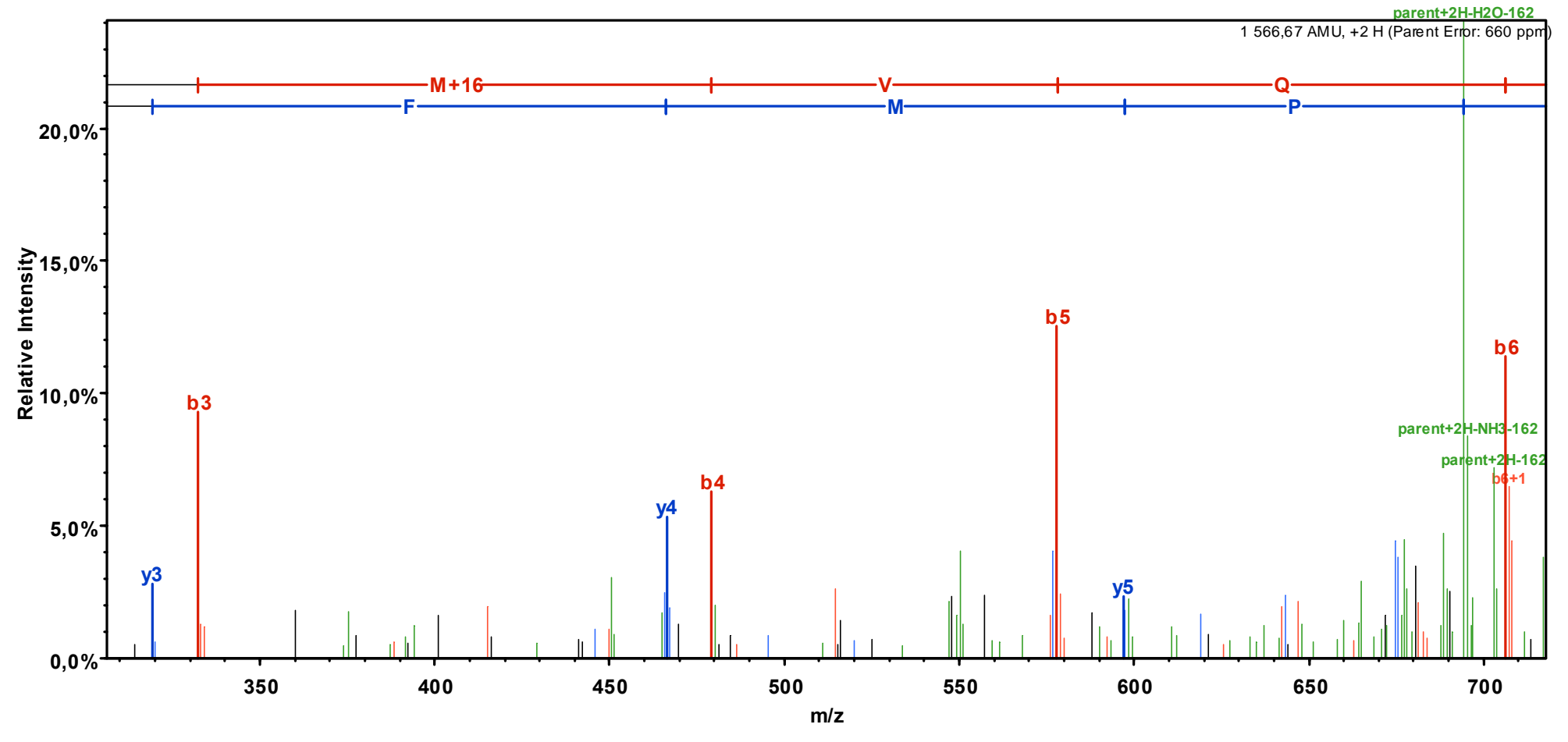

\section{$\underline{\text { Right of precursor }}$}

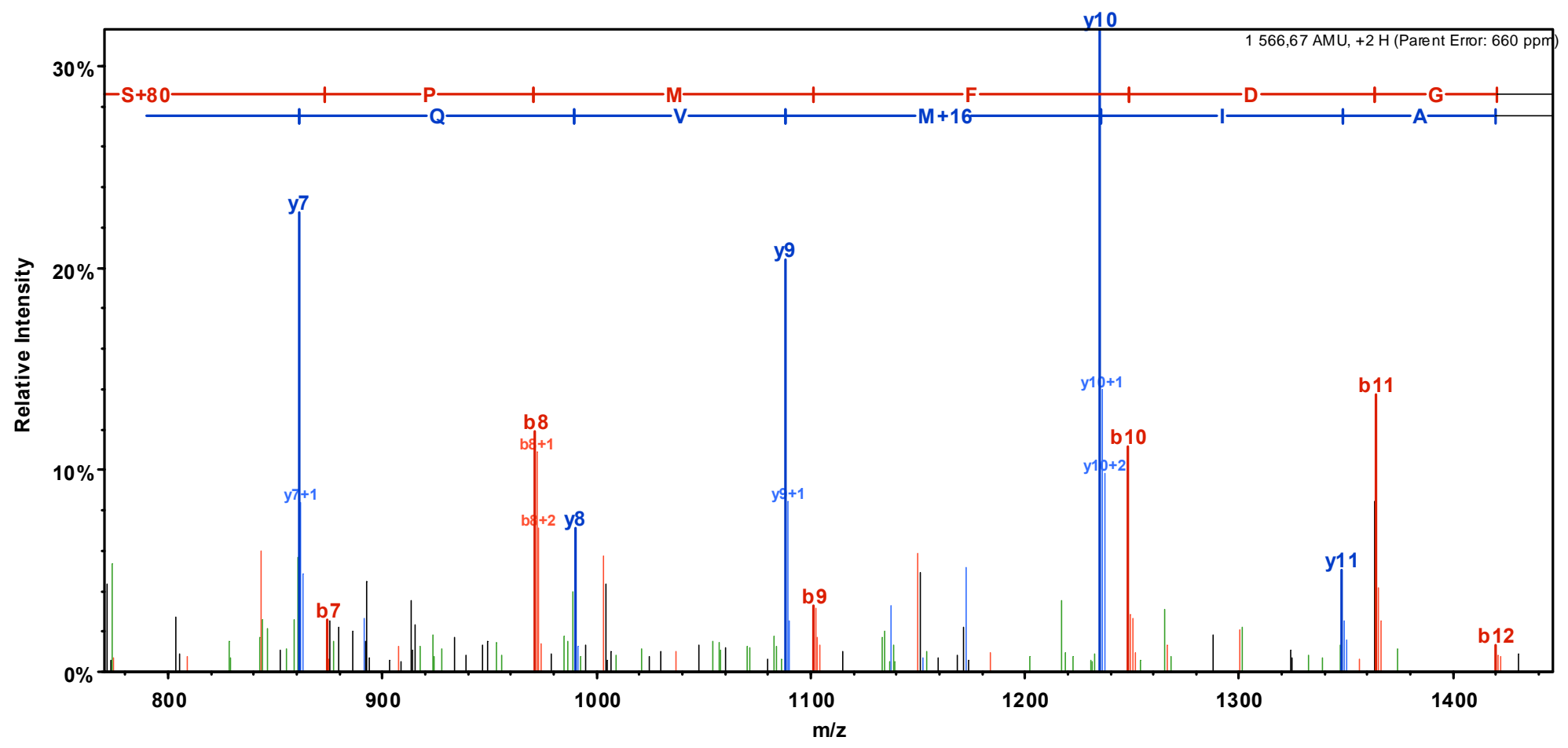




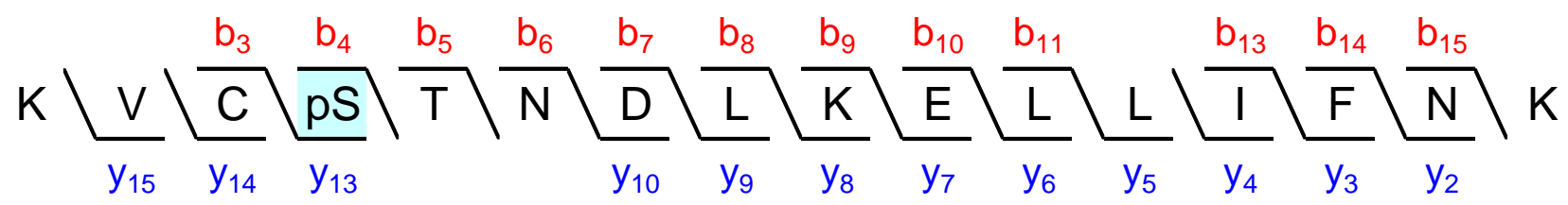

Sequest: $X$ Corr $=3.85, \mathrm{dCn} 1=0.05$ (between $1^{\text {st }}$ and $2^{\text {nd }}$ best matches), $\mathrm{dCn} 2=0.66$ (between $2^{\text {nd }}$ and $3^{\text {rd }}$ best matches) . $A_{\text {score }}=0$.

Sequest's second best match with XCorr $=3.65$ is to the same peptide sequence but with an alternative phosphorylation site: KVCSPTNDLKELLIFNK.

The very weak Ascore indicates that, given the observed MS/MS spectrum, the phosphopeptide identified could either be Sequest's first or second best match (Nat Biotechnol. 2006;24(10):1285-92).

\section{MS/MS spectrum's fragmentation table}

\begin{tabular}{|c|c|c|c|c|c|c|c|c|c|c|}
\hline B & B Ions & $\mathrm{B}+2 \mathrm{H}$ & $\mathrm{B}-\mathrm{NH} 3$ & $\mathrm{~B}-\mathrm{H} 2 \mathrm{O}$ & AA & $Y$ Ions & $\mathrm{Y}+2 \mathrm{H}$ & $\mathrm{Y}-\mathrm{NH} 3$ & $\mathrm{Y}-\mathrm{H} 2 \mathrm{O}$ & $Y$ \\
\hline 1 & 129,1 & 65.1 & 112,1 & & $\mathrm{~K}$ & 2002,0 & 1001,5 & 1985,0 & 1984,0 & 16 \\
\hline 2 & 228,2 & 114,6 & 211,1 & & V & 1873,9 & 937.5 & 1856,9 & 1855,9 & 15 \\
\hline 3 & 388.2 & 194,6 & 371,2 & & $C+57$ & 1774,8 & 887.9 & 1757,8 & 1756,8 & 14 \\
\hline 4 & 475,2 & 238,1 & 458.2 & 457.2 & S & 1614,8 & 807.9 & 1597,8 & 1596,8 & 13 \\
\hline 5 & 656.2 & 328.6 & 639.2 & 638.2 & $\mathbf{T}+\mathbf{8 0}$ & 1527,8 & 764,4 & 1510,8 & 1509,8 & 12 \\
\hline 6 & 770,3 & 385,6 & 753.3 & 752.3 & $\mathrm{~N}$ & 1346,8 & 673,9 & 1329,7 & 1328,8 & 11 \\
\hline 7 & 885.3 & 443.2 & 868.3 & 867.3 & D & 1232,7 & 616.9 & 1215.7 & 1214,7 & 10 \\
\hline 8 & 998,4 & 499,7 & 981,4 & 980,4 & L & 1117,7 & 559.4 & 1100,7 & 1099,7 & 9 \\
\hline 9 & 1126.5 & 563.8 & 1109,5 & 1108,5 & K & 1004.6 & 502,8 & 987.6 & 986.6 & 8 \\
\hline 10 & 1255,5 & 628.3 & 1238,5 & 1237,5 & E & 876.5 & 438,8 & 859.5 & 858,5 & 7 \\
\hline 11 & 1368,6 & 684.8 & 1351,6 & 1350,6 & L & 747.5 & 374,2 & 730.5 & & 6 \\
\hline 12 & 1481,7 & 741,4 & 1464,7 & 1463,7 & L & 634.4 & & 617,4 & & 5 \\
\hline 13 & 1594,8 & 797.9 & 1577,8 & 1576,8 & I & 521.3 & & 504.3 & & 4 \\
\hline 14 & 1741,9 & 871.4 & 1724,8 & 1723,8 & $F$ & 408.2 & & 391,2 & & 3 \\
\hline 15 & 1855,9 & 928.5 & 1838,9 & 1837,9 & $\mathrm{~N}$ & 261.2 & & 244.1 & & 2 \\
\hline 16 & 2002,0 & 1001,5 & 1985,0 & 1984,0 & K & 147,1 & & 130,1 & & 1 \\
\hline
\end{tabular}




\section{MS/MS spectrum}

\section{Full-range}

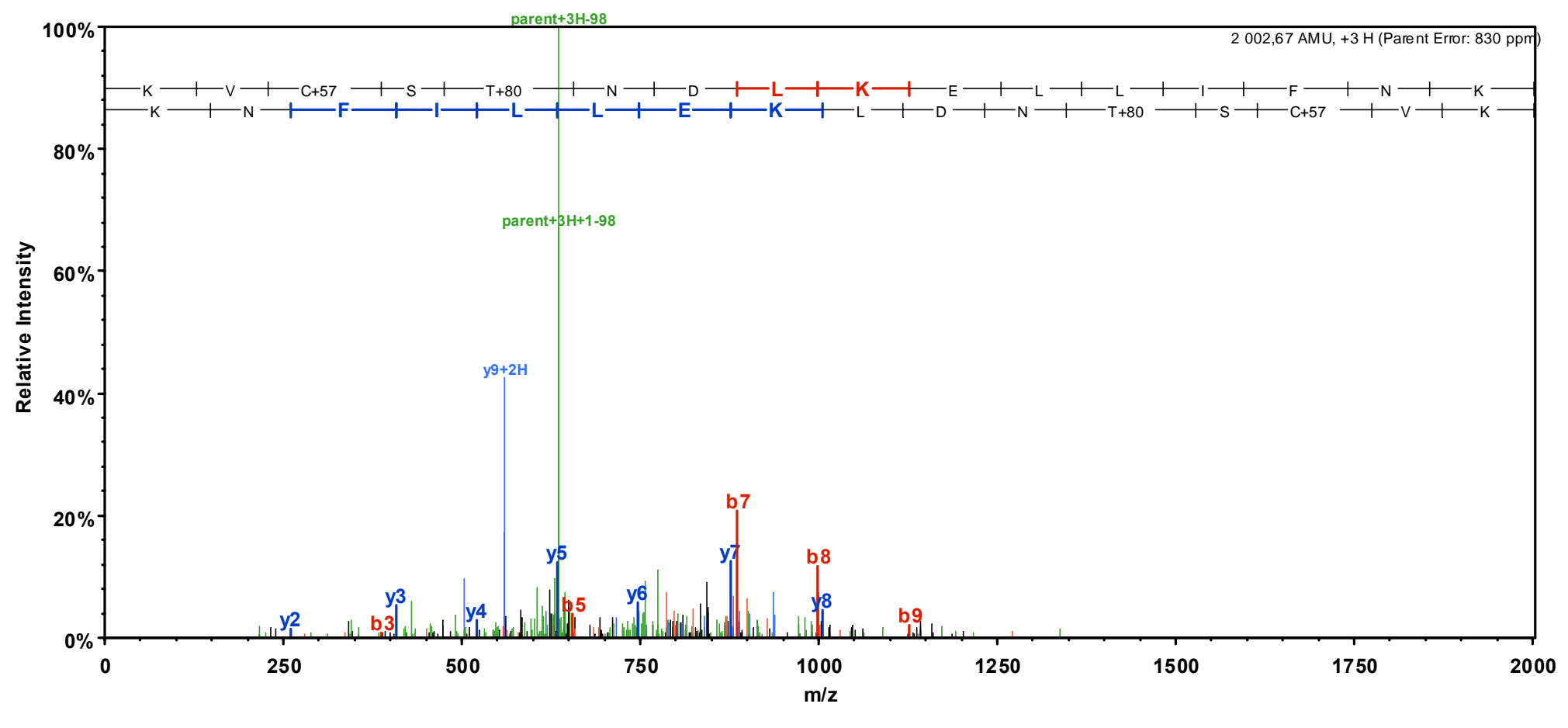

\section{$\underline{\text { Close to precursor }}$}

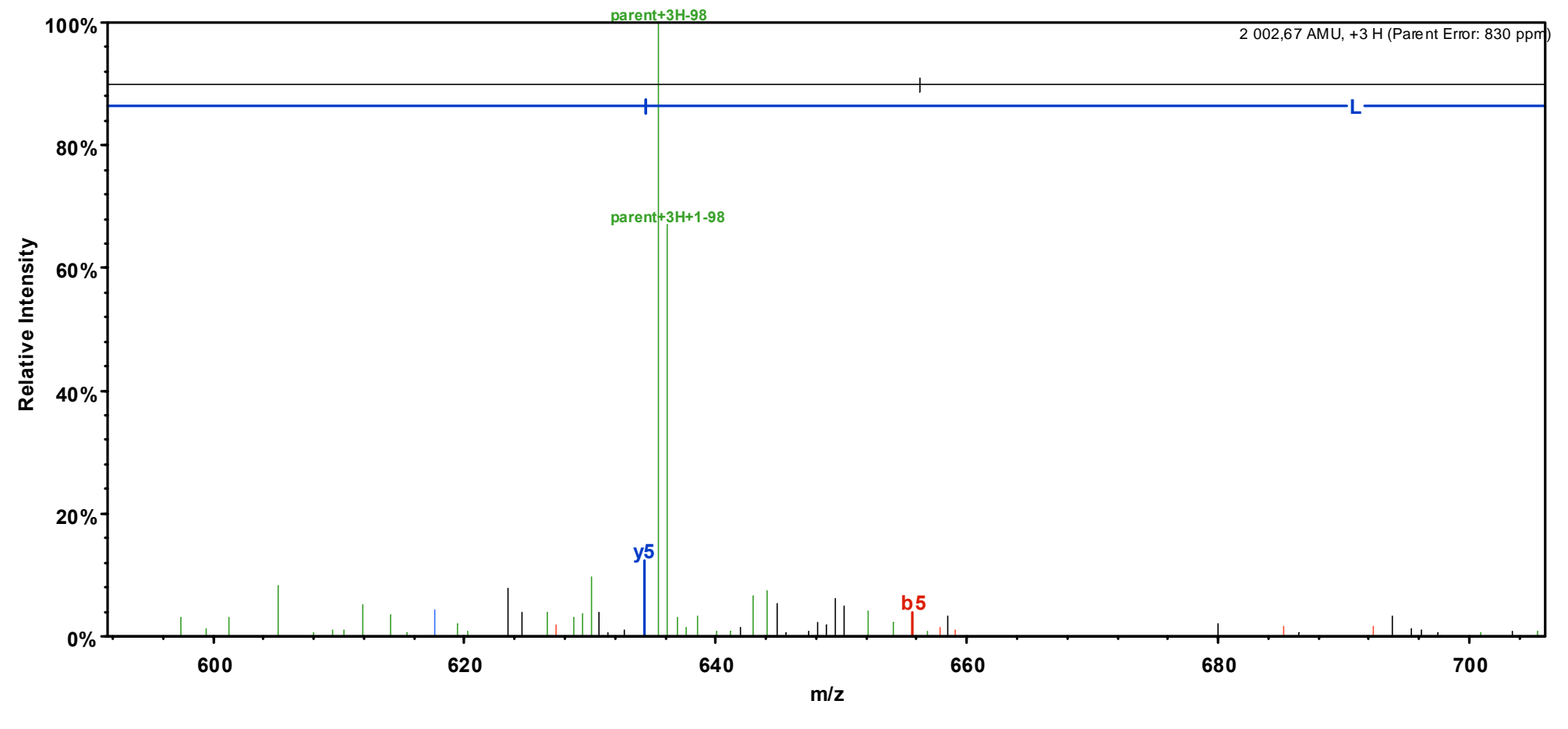




\section{Left of precursor}

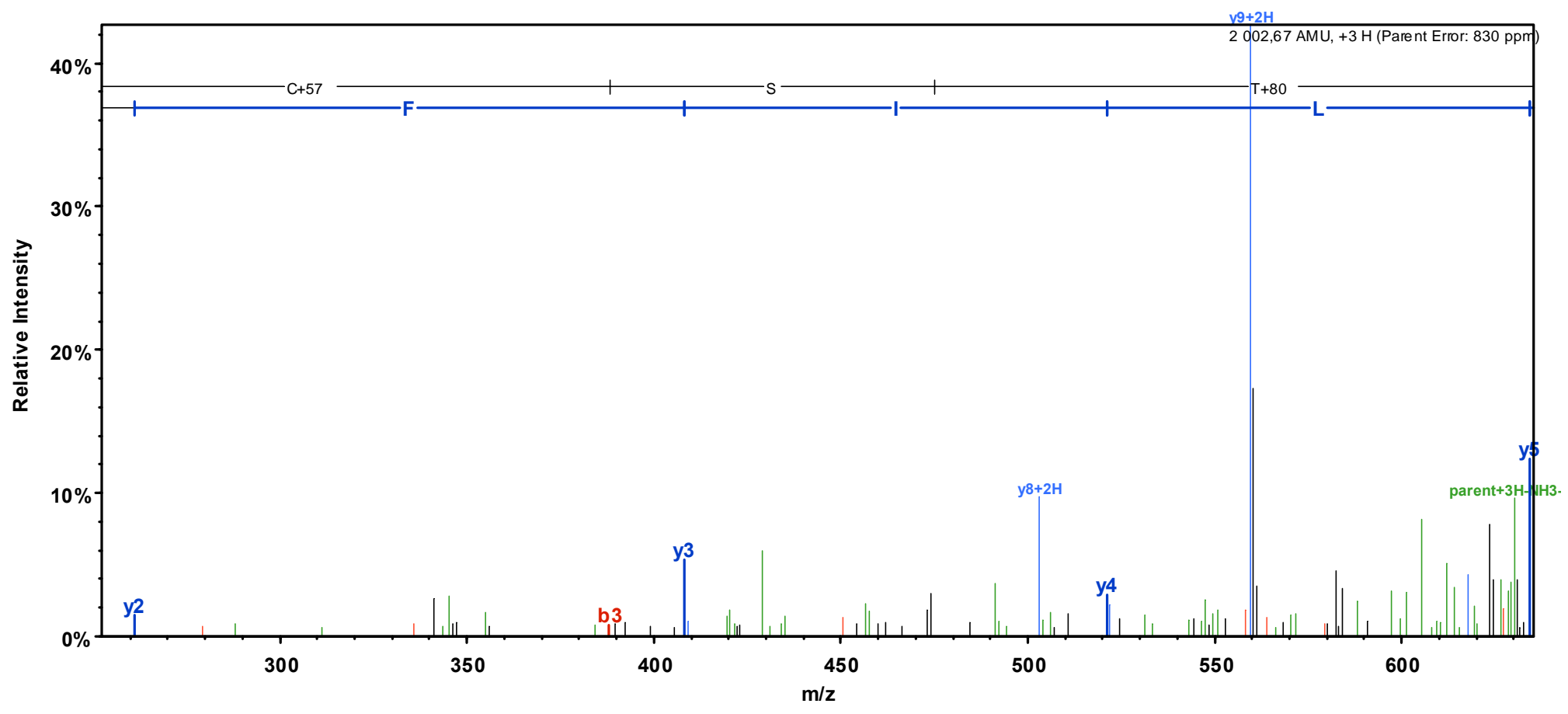

\section{$\underline{\text { Right of precursor }}$}

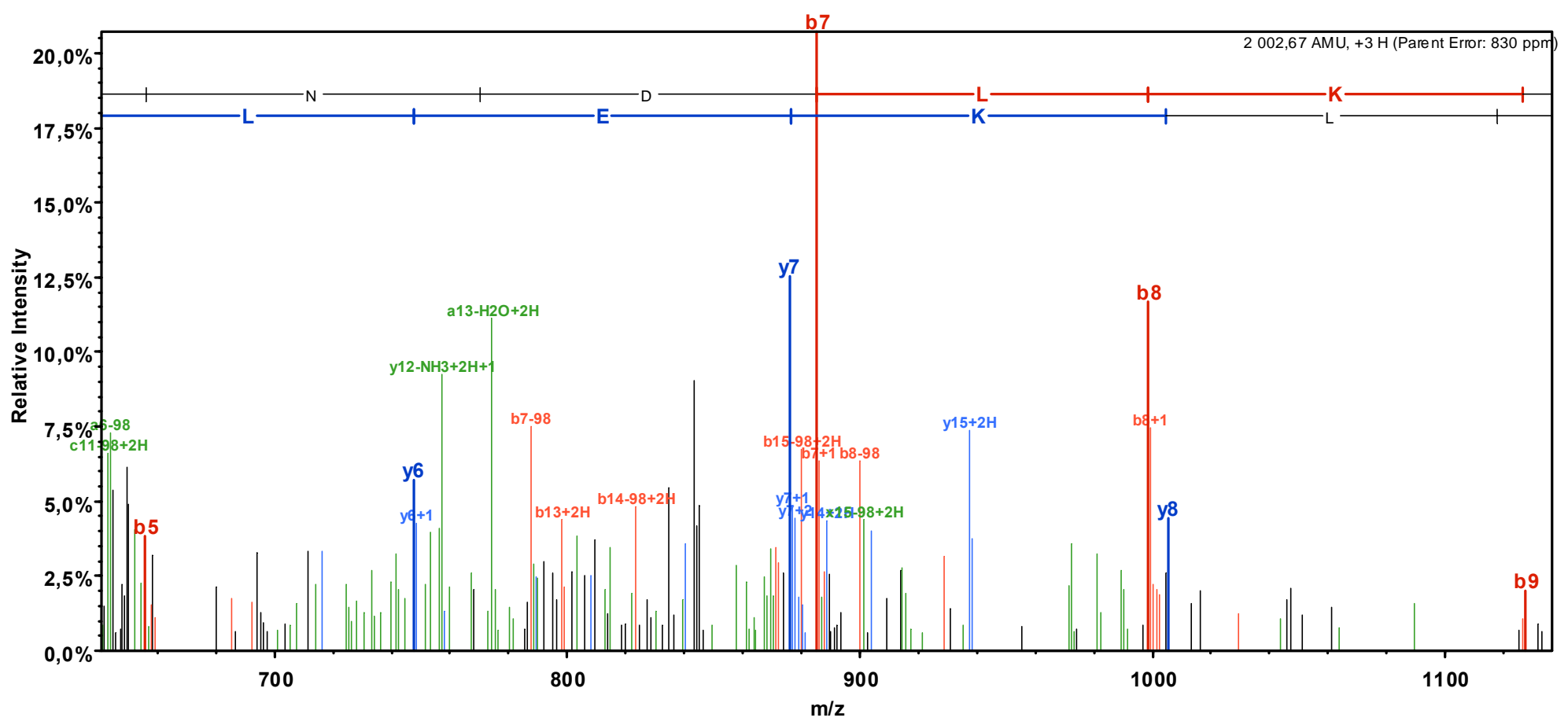




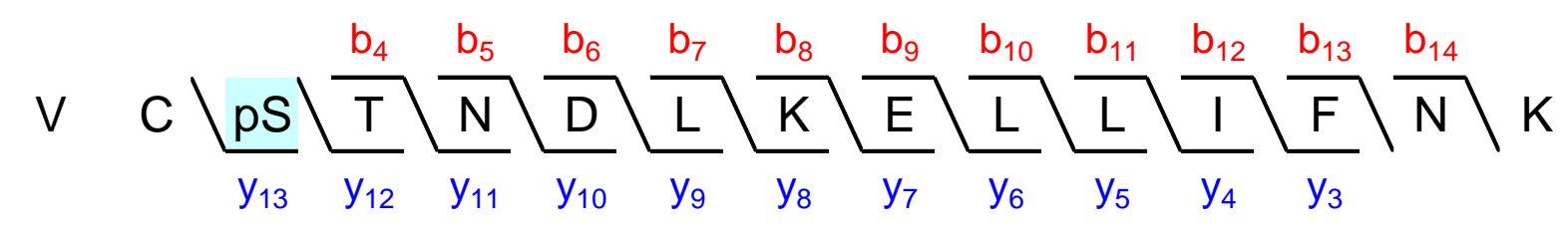

Sequest: $X$ Corr $=3.10, \mathrm{dCn} 1=0.08$ (between $1^{\text {st }}$ and $2^{\text {nd }}$ best matches), $\mathrm{dCn} 2=0.49$ (between $2^{\text {nd }}$ and $3^{\text {rd }}$ best matches). $A_{\text {score }}=9.34$.

Sequest's second best match with XCorr $=2.85$ is to the same peptide sequence but with an alternative phosphorylation site: VCSPTNDLKELLIFNK.

The very weak Ascore indicates that, given the observed MS/MS spectrum, the phosphopeptide identified could either be Sequest's first or second best match (Nat Biotechnol. 2006;24(10):1285-92).

\section{MS/MS spectrum's fragmentation table}

\begin{tabular}{|c|c|c|c|c|c|c|c|c|c|c|}
\hline B & B Ions & $\mathrm{B}+2 \mathrm{H}$ & $\mathrm{B}-\mathrm{NH} 3$ & $\mathrm{~B}-\mathrm{H} 2 \mathrm{O}$ & $\mathrm{AA}$ & $Y$ Ions & $\mathrm{Y}+2 \mathrm{H}$ & $\mathrm{Y}-\mathrm{NH} 3$ & $\mathrm{Y}-\mathrm{H} 2 \mathrm{O}$ & Y \\
\hline 1 & 100,1 & & & & $\mathrm{~V}$ & 1873,9 & 937,5 & 1856.9 & 1855,9 & 15 \\
\hline 2 & 260,1 & & & & $C+57$ & 1774,8 & 887,9 & 1757,8 & 1756,8 & 14 \\
\hline 3 & 427,1 & & & 409,1 & $S+80$ & 1614,8 & $\mathbf{8 0 7 . 9}$ & 1597.8 & 1596,8 & 13 \\
\hline 4 & 528.2 & & & 510,1 & $\mathbf{T}$ & 1447.8 & 724.4 & 1430,8 & 1429,8 & 12 \\
\hline 5 & 642.2 & & 625,2 & 624,2 & $\mathrm{~N}$ & 1346.8 & 673.9 & 1329,7 & 1328.8 & 11 \\
\hline 6 & 757.2 & 379,1 & 740.2 & 739,2 & D & 1232.7 & 616.9 & 1215.7 & 1214,7 & 10 \\
\hline 7 & 870.3 & 435,7 & 853.3 & 852,3 & L & 1117.7 & 559,4 & 1100,7 & 1099,7 & 9 \\
\hline 8 & 998.4 & 499,7 & 981,4 & 980.4 & $\mathrm{~K}$ & 1004.6 & 502,8 & 987,6 & 986,6 & 8 \\
\hline 9 & 1127.4 & 564,2 & 1110.4 & 1109,4 & $E$ & 876.5 & 438,8 & 859,5 & 858,5 & 7 \\
\hline 10 & 1240,5 & 620,8 & 1223.5 & 1222,5 & $\mathbf{L}$ & 747,5 & 374.2 & 730,5 & & 6 \\
\hline 11 & 1353.6 & 677,3 & 1336,6 & 1335.6 & L & 634.4 & & 617.4 & & 5 \\
\hline 12 & 1466.7 & 733.9 & 1449,7 & 1448,7 & I & 521,3 & & 504.3 & & 4 \\
\hline 13 & 1613,8 & 807.4 & 1596.7 & 1595,8 & $F$ & 408.2 & & 391,2 & & 3 \\
\hline 14 & 1727,8 & 864.4 & 1710,8 & 1709,8 & $\mathbf{N}$ & 261,2 & & 244,1 & & 2 \\
\hline 15 & 1873,9 & 937,5 & 1856,9 & 1855,9 & K & 147,1 & & 130,1 & & 1 \\
\hline
\end{tabular}




\section{MS/MS spectrum}

\section{Full-range}

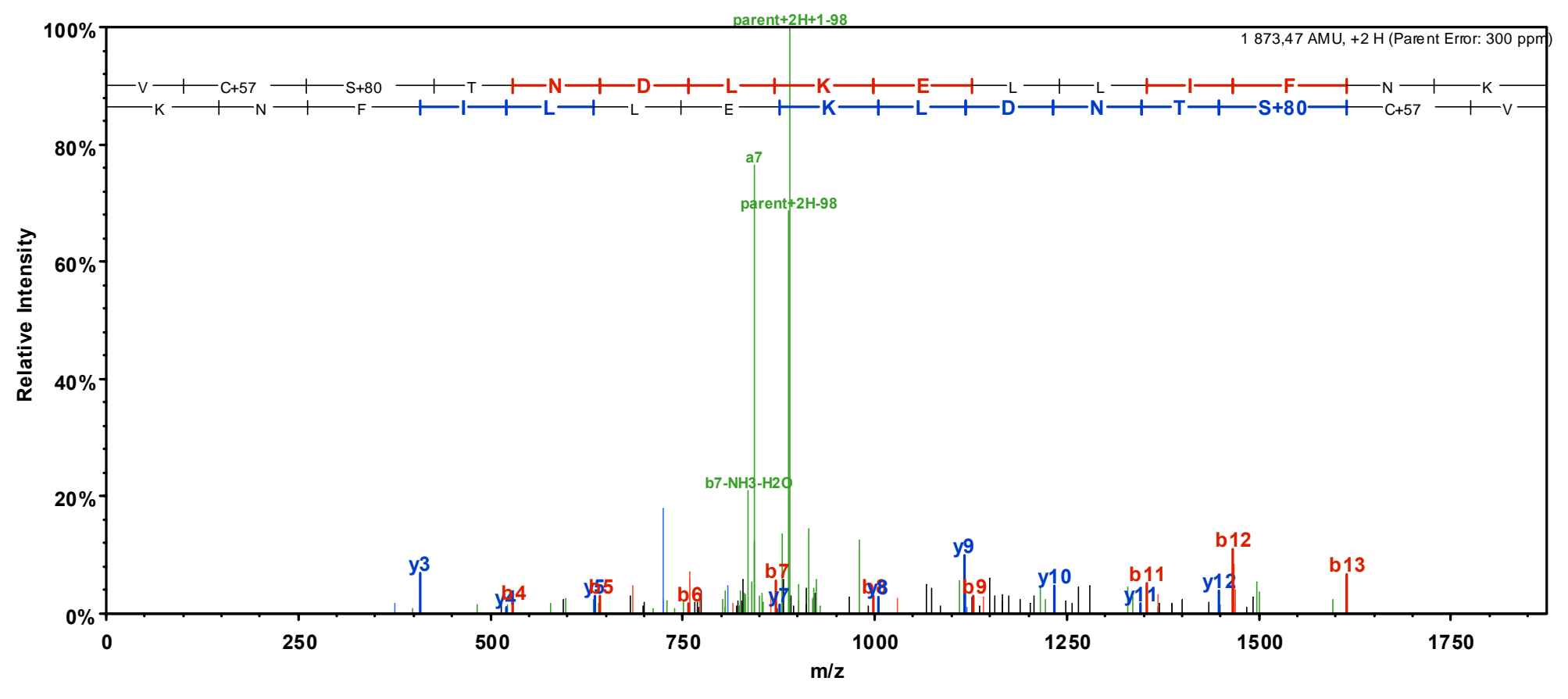

\section{$\underline{\text { Close to precursor }}$}

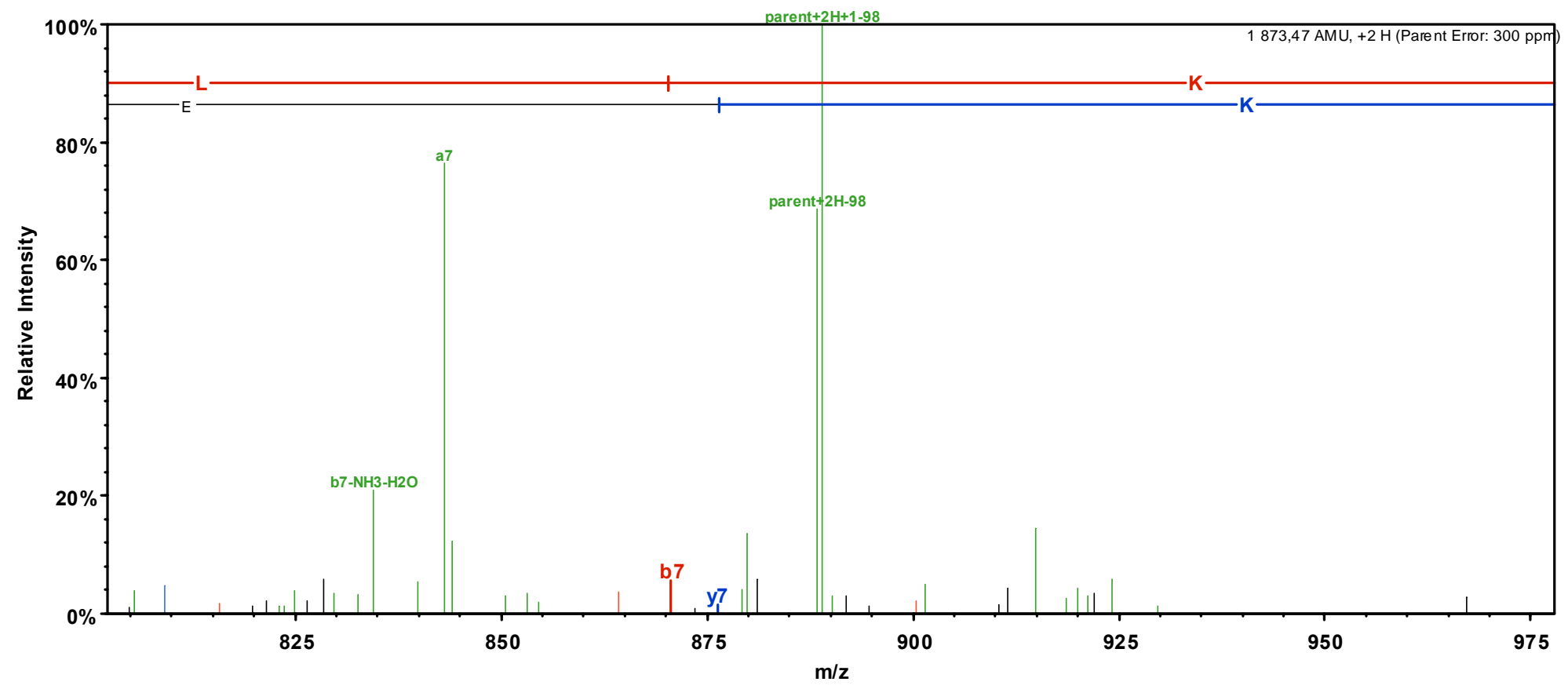




\section{Left of precursor}

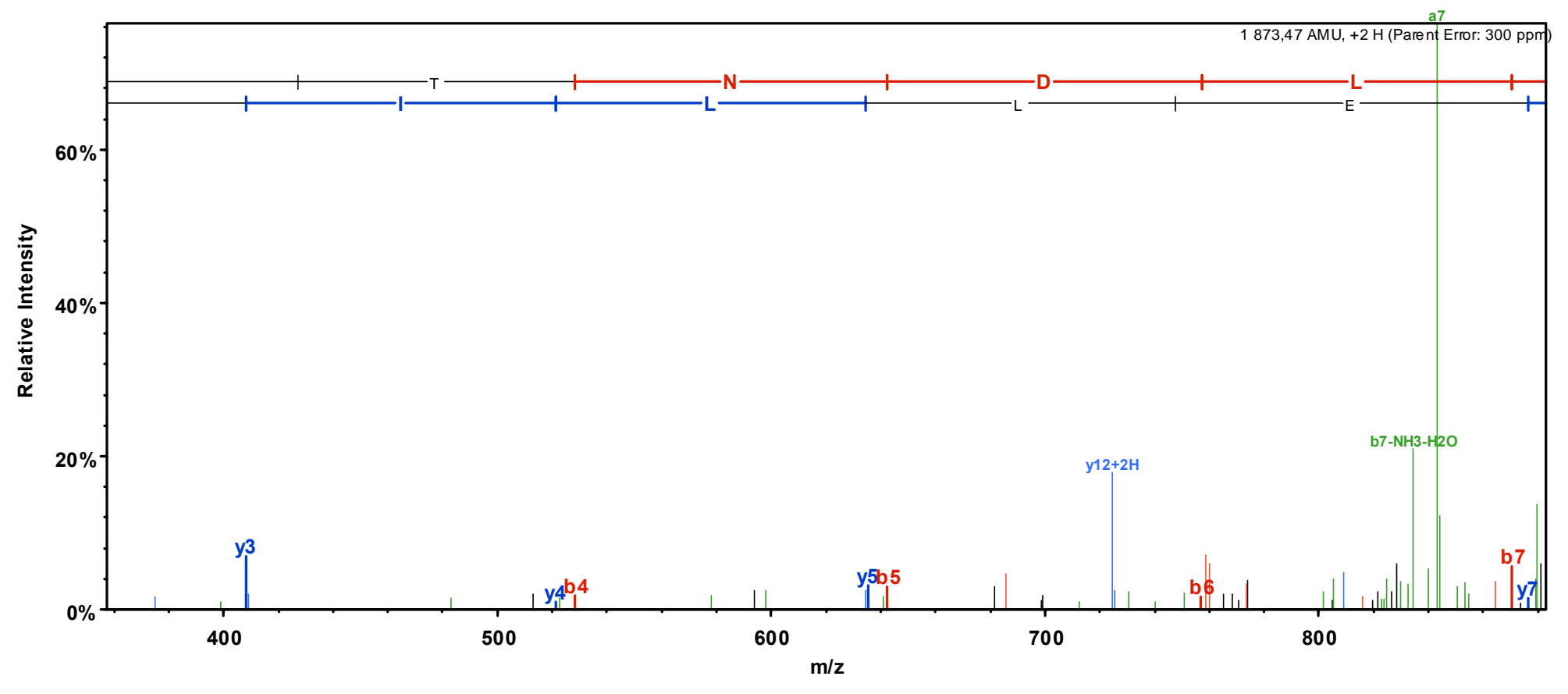

\section{$\underline{\text { Right of precursor }}$}

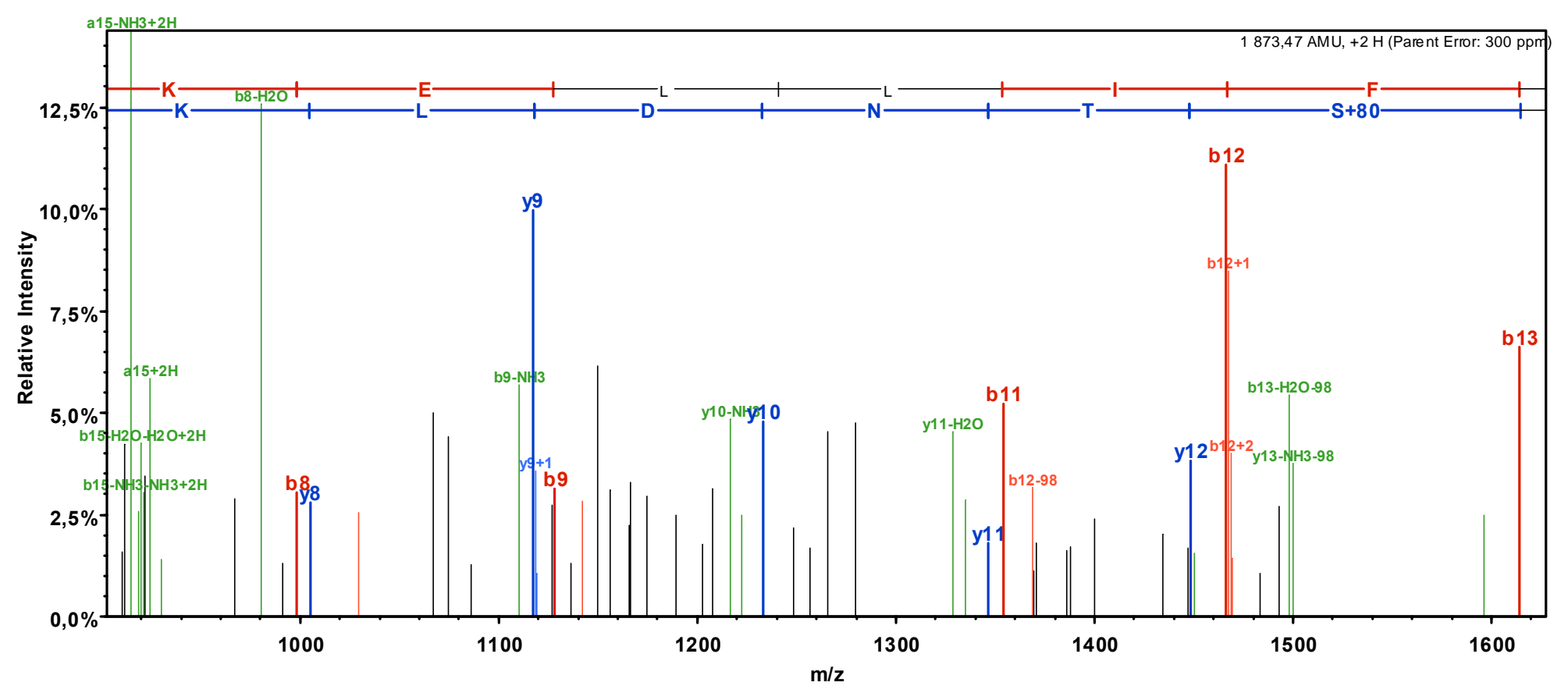




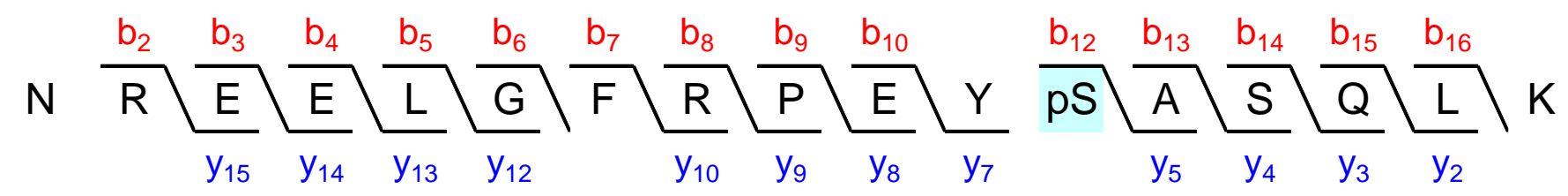

Sequest: $\mathrm{XCorr}=2.89, \mathrm{dCn} 1=0.003$ (between $1^{\text {st }}$ and $2^{\text {nd }}$ best matches), $\mathrm{dCn} 2=0.21$ (between $2^{\text {nd }}$ and $3^{\text {rd }}$ best matches) . $A_{\text {score }}=0$.

Sequest's second best match with XCorr $=2.89$ is to the same peptide sequence but with an alternative phosphorylation site: NREELGFRPEPYSASQLK.

The very weak Ascore indicates that, given the observed MS/MS spectrum, the phosphopeptide identified could either be Sequest's first or second best match (Nat Biotechnol. 2006;24(10):1285-92). However, since a strong neutral loss of the phosphate ion is observed, the phosphotyrosine peptide can be ruled out since phosphotyrosine do not undergo neutral loss.

\section{MS/MS spectrum's fragmentation table}

\begin{tabular}{|c|c|c|c|c|c|c|c|c|c|c|}
\hline B & B Ions & $\mathrm{B}+2 \mathrm{H}$ & $\mathrm{B}-\mathrm{NH} 3$ & $\mathrm{~B}-\mathrm{H} 2 \mathrm{O}$ & AA & $Y$ Ions & $\mathrm{Y}+2 \mathrm{H}$ & $\mathrm{Y}-\mathrm{NH} 3$ & $\mathrm{Y}-\mathrm{H} 2 \mathrm{O}$ & $Y$ \\
\hline 1 & 115,1 & & 98,0 & & $\mathbf{N}$ & 2104,0 & 1052,5 & 2087,0 & 2086,0 & 17 \\
\hline 2 & 271.2 & 136.1 & 254,1 & & $\mathbf{R}$ & 1989,9 & 995,5 & 1972,9 & 1971,9 & 16 \\
\hline 3 & 400.2 & 200,6 & 383,2 & 382,2 & $E$ & 1833,8 & 917.4 & 1816,8 & 1815,8 & 15 \\
\hline 4 & 529.2 & 265,1 & 512,2 & 511,2 & E & 1704,8 & 852.9 & 1687,8 & 1686,8 & 14 \\
\hline 5 & 642.3 & 321,7 & 625.3 & 624,3 & L & 1575,8 & 788.4 & 1558.7 & 1557,7 & 13 \\
\hline 6 & 699,3 & 350,2 & 682,3 & 681,3 & G & 1462,7 & 731.8 & 1445,6 & 1444,7 & 12 \\
\hline 7 & 846.4 & 423.7 & 829.4 & 828,4 & $F$ & 1405,7 & 703,3 & 1388.6 & 1387,6 & 11 \\
\hline 8 & 1002.5 & 501,8 & 985.5 & 984,5 & $\mathbf{R}$ & 1258,6 & 629.8 & 1241,6 & 1240,6 & 10 \\
\hline 9 & 1099.6 & 550.3 & 1082,5 & 1081,6 & $\mathbf{P}$ & 1102.5 & 551.7 & 1085,5 & 1084,5 & 9 \\
\hline 10 & 1228.6 & 614,8 & 1211.6 & 1210,6 & $\mathrm{E}$ & 1005.4 & 503.2 & 988.4 & 987.4 & 8 \\
\hline 11 & 1391,7 & 696,3 & 1374,6 & 1373,7 & $\mathbf{Y}$ & 876.4 & 438,7 & 859.4 & $\mathbf{8 5 8 . 4}$ & 7 \\
\hline 12 & 1558.7 & 779.8 & 1541,6 & 1540,7 & $S+80$ & 713,3 & 357,2 & 696,3 & 695,3 & 6 \\
\hline 13 & 1629,7 & 815,4 & 1612,7 & 1611,7 & A & 546.3 & & 529,3 & 528,3 & 5 \\
\hline 14 & 1716.7 & 858.9 & 1699,7 & 1698,7 & S & 475.3 & & 458,3 & 457,3 & 4 \\
\hline 15 & 1844,8 & 922.9 & 1827,8 & 1826,8 & Q & 388.3 & & 371.2 & & 3 \\
\hline 16 & 1957,9 & 979.4 & 1940,9 & 1939,9 & L & 260.2 & & 243,2 & & 2 \\
\hline 17 & 2104,0 & 1052,5 & 2087,0 & 2086.0 & K & 147,1 & & 130,1 & & 1 \\
\hline
\end{tabular}




\section{MS/MS spectrum}

\section{Full-range}

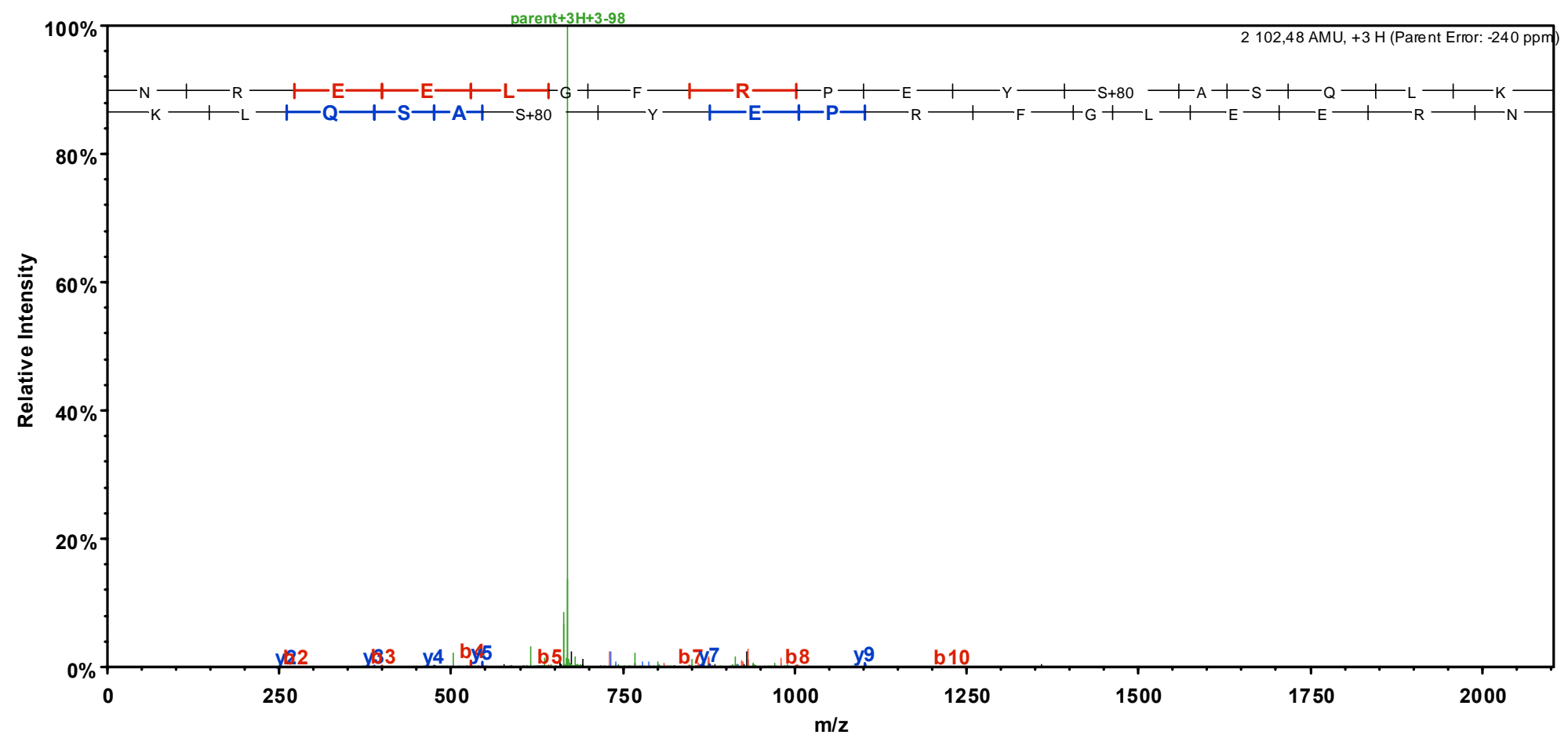

\section{$\underline{\text { Close to precursor }}$}

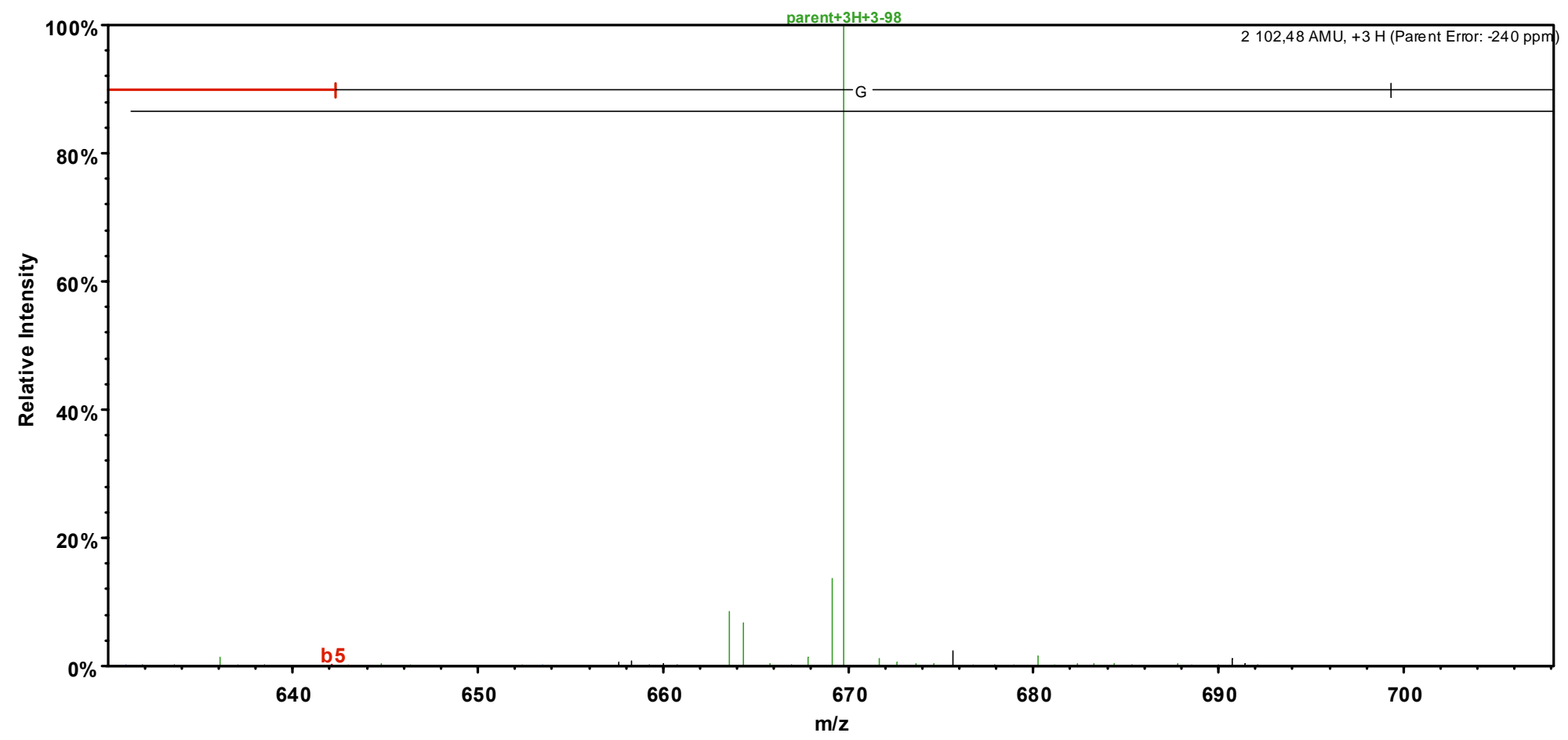




\section{Left of precursor}

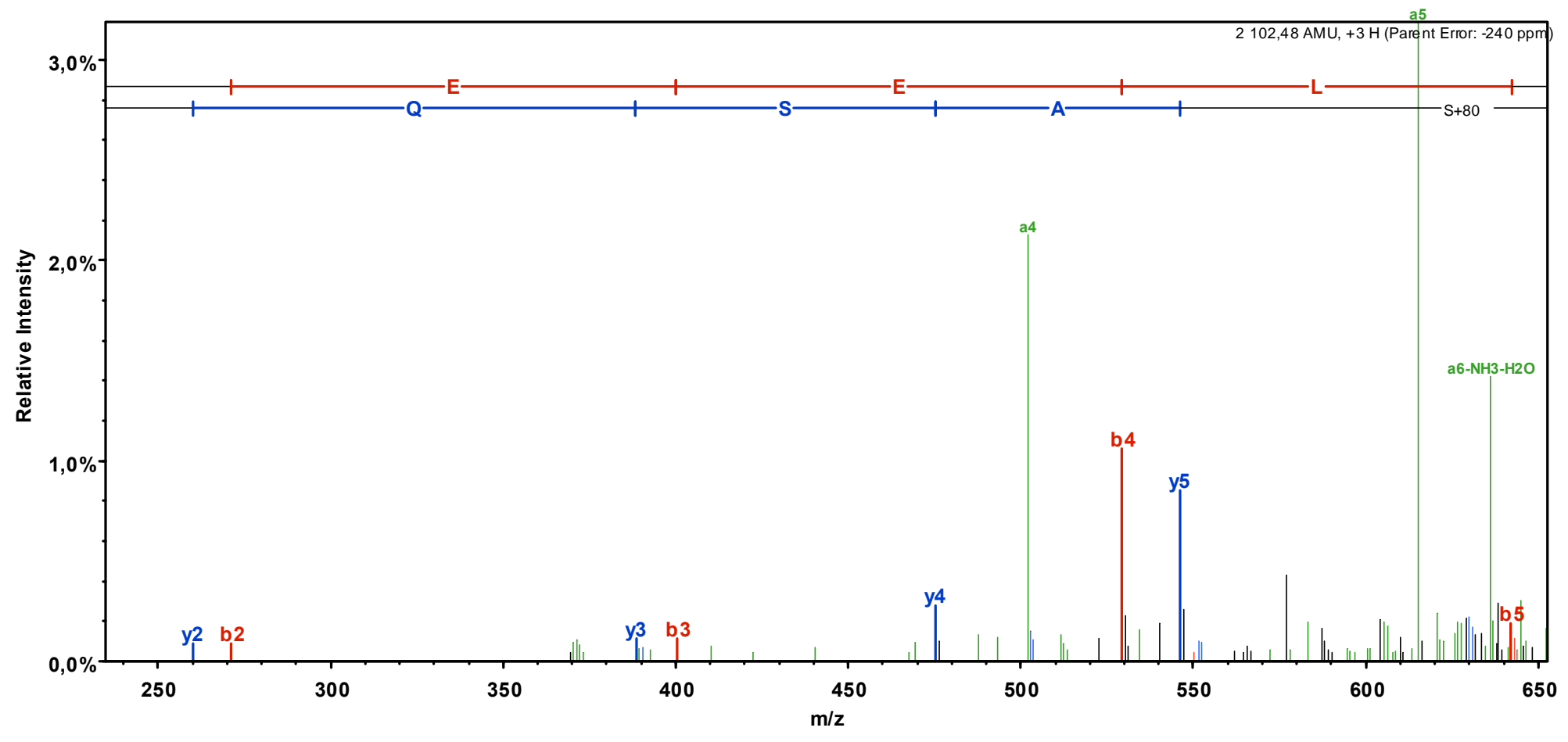

\section{$\underline{\text { Right of precursor }}$}

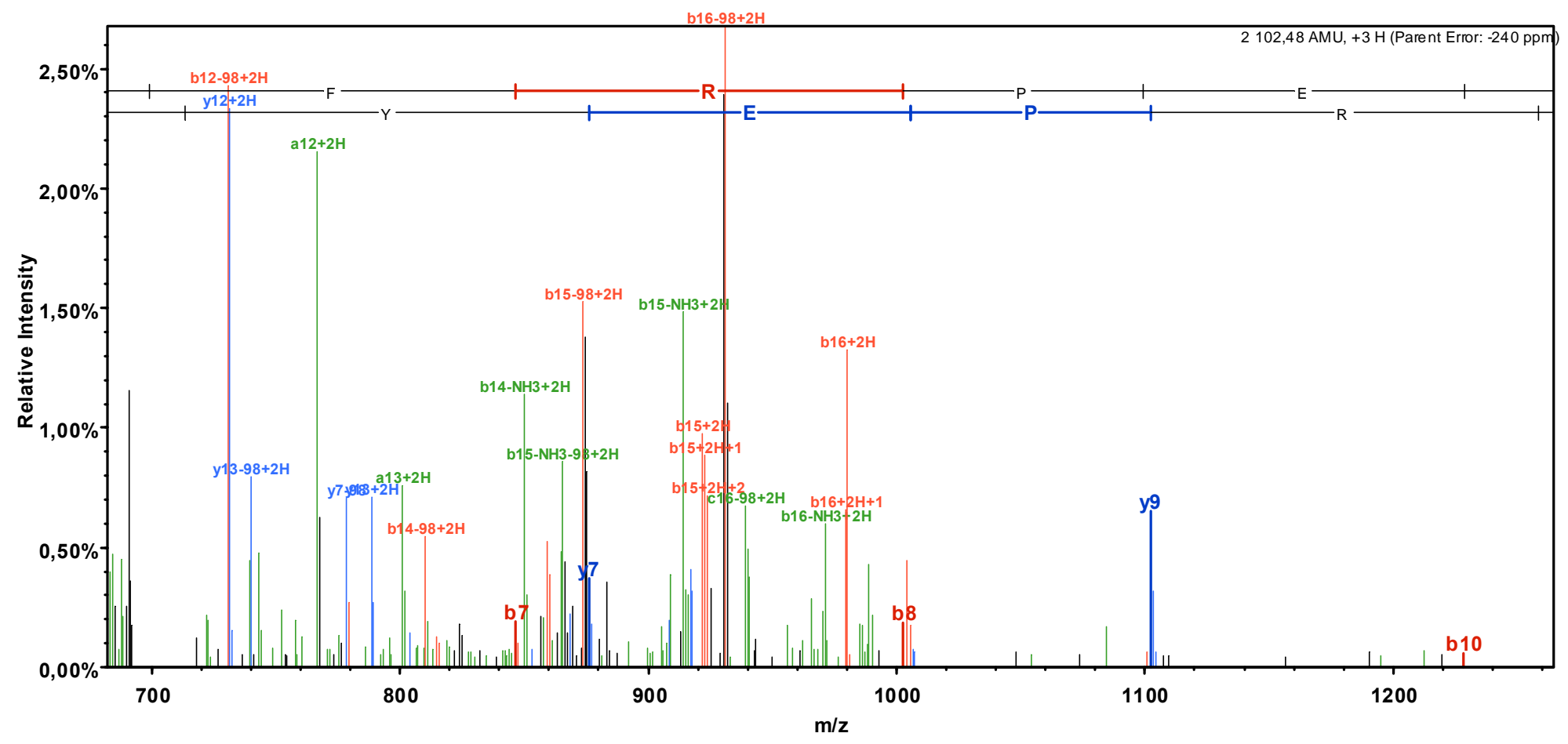




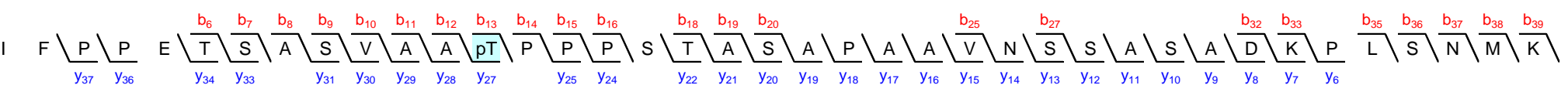

Sequest: $X$ Corr $=4.30, \mathrm{dCn} 1=0.03$ (between $1^{\text {st }}$ and $2^{\text {nd }}$ best matches), $\mathrm{dCn} 2=0.03$ (between $2^{\text {nd }}$ and $3^{\text {rd }}$ best matches), $\mathrm{dCn} 3=0.03$ (between $3^{\text {rd }}$ and $4^{\text {th }}$ best matches), $0.17 \mathrm{dCn} 4=$ (between $4^{\text {th }}$ and $5^{\text {th }}$ best matches).

Ascore $=16.10$.

Sequest's second, third and fourth best matches with XCorr $=4.18,4.18$ and 4.17 respectively are to the same peptide sequence but with alternative phosphorylation sites:
IFPPETSASVAATPPPSPTASAPAAVNSSASADKPLSNMK
IFPPETSASVAATPPPPSTASAPAAVNSSASADKPLSNMK
IFPPETSASVAATPPPSTAPSAPAAVNSSASADKPLSNMK

The weak Ascore indicates that, given the observed MS/MS spectrum, the phosphopeptide identified could be either of the above four (Nat Biotechnol. 2006;24(10):1285-92).

\section{MS/MS spectrum's fragmentation table}

\begin{tabular}{|c|c|c|c|c|c|c|c|c|c|c|}
\hline B & B Ions & $\mathrm{B}+2 \mathrm{H}$ & $\mathrm{B}-\mathrm{NH} 3$ & $\mathrm{~B}-\mathrm{H} 2 \mathrm{O}$ & AA & $Y$ Ions & $\mathrm{Y}+2 \mathrm{H}$ & $\mathrm{Y}-\mathrm{NH} 3$ & $\mathrm{Y}-\mathrm{H} 2 \mathrm{O}$ & $Y$ \\
\hline 1 & 114,1 & & & & I & 3863,8 & 1932,4 & 3846,8 & 3845,8 & 39 \\
\hline 2 & 261,2 & & & & $F$ & 3750,8 & 1875,9 & 3733,7 & 3732,7 & 38 \\
\hline 3 & 358,2 & & & & $\mathbf{P}$ & 3603.7 & 1802.3 & 3586.7 & 3585,7 & 37 \\
\hline 4 & 455,3 & & & & $\mathbf{P}$ & 3506,6 & 1753.8 & 3489,6 & 3488,6 & 36 \\
\hline 5 & 584,3 & & & 566,3 & $E$ & 3409,6 & 1705.3 & 3392,6 & 3391,6 & 35 \\
\hline 6 & 685.4 & 343,2 & & 667.3 & $\mathbf{T}$ & 3280,5 & 1640.8 & 3263,5 & 3262,5 & 34 \\
\hline 7 & 772.4 & 386,7 & & 754,4 & S & 3179,5 & 1590.3 & 3162,5 & 3161,5 & 33 \\
\hline 8 & 843.4 & 422,2 & & 825,4 & A & 3092,5 & 1546,7 & 3075,4 & 3074,5 & 32 \\
\hline 9 & 930.5 & 465,7 & & 912.4 & S & 3021.4 & 1511.2 & 3004.4 & 3003.4 & 31 \\
\hline 10 & 1029.5 & 515,3 & & 1011.5 & $\mathrm{~V}$ & 2934,4 & 1467.7 & 2917,4 & 2916.4 & 30 \\
\hline 11 & 1100.6 & 550,8 & & 1082.6 & A & 2835,3 & 1418.2 & 2818,3 & 2817,3 & 29 \\
\hline 12 & 1171.6 & 586,3 & & 1153.6 & A & 2764,3 & 1382.6 & 2747,3 & 2746,3 & 28 \\
\hline 13 & 1352.6 & 676.8 & & 1334.6 & $\mathbf{T}+\mathbf{8 0}$ & 2693,2 & 1347.1 & 2676,2 & 2675,2 & 27 \\
\hline 14 & 1449.7 & 725,3 & & 1431,7 & $\mathbf{P}$ & 2512,2 & 1256.6 & 2495,2 & 2494,2 & 26 \\
\hline 15 & 1546.7 & 773,9 & & 1528,7 & $\mathbf{P}$ & 2415,2 & 1208.1 & 2398,2 & 2397,2 & 25 \\
\hline 16 & 1643.8 & 822.4 & & 1625,8 & $\mathbf{P}$ & 2318,1 & 1159.6 & 2301,1 & 2300,1 & 24 \\
\hline 17 & 1730,8 & 865,9 & & 1712,8 & $S$ & 2221,1 & 1111.0 & 2204,1 & 2203.1 & 23 \\
\hline 18 & 1831,9 & 916.4 & & 1813,8 & $\mathbf{T}$ & 2134,0 & 1067.5 & 2117.0 & 2116,0 & 22 \\
\hline 19 & 1902.9 & 951,9 & & 1884,9 & A & 2033,0 & 1017.0 & 2016.0 & 2015,0 & 21 \\
\hline 20 & 1989.9 & 995.5 & & 1971,9 & S & 1962.0 & 981.5 & 1944,9 & 1943,9 & 20 \\
\hline 21 & 2061,0 & 1031,0 & & 2042,9 & A & 1874.9 & 938.0 & 1857,9 & 1856.9 & 19 \\
\hline 22 & 2158,0 & 1079.5 & & 2140,0 & $\mathbf{P}$ & 1803.9 & 902.4 & 1786,9 & 1785,9 & 18 \\
\hline 23 & 2229,0 & 1115,0 & & 2211,0 & A & 1706,8 & 853.9 & 1689,8 & 1688.8 & 17 \\
\hline 24 & 2300,1 & 1150,5 & & 2282,1 & A & 1635,8 & 818.4 & 1618,8 & 1617,8 & 16 \\
\hline 25 & 2399,2 & 1200.1 & & 2381,1 & V & 1564.8 & 782.9 & 1547,7 & 1546,8 & 15 \\
\hline 26 & 2513,2 & 1257,1 & 2496,2 & 2495,2 & $\mathrm{~N}$ & 1465.7 & 733.4 & 1448,7 & 1447,7 & 14 \\
\hline 27 & 2600,2 & 1300.6 & 2583,2 & 2582,2 & S & 1351.7 & 676,3 & 1334,6 & 1333.6 & 13 \\
\hline 28 & 2687,3 & 1344,1 & 2670,2 & 2669,2 & S & 1264.6 & 632,8 & 1247.6 & 1246,6 & 12 \\
\hline 29 & 2758,3 & 1379,7 & 2741,3 & 2740,3 & A & 1177.6 & 589,3 & 1160.6 & 1159,6 & 11 \\
\hline 30 & 2845,3 & 1423,2 & 2828,3 & 2827,3 & S & 1106.6 & 553,8 & 1089.5 & 1088,5 & 10 \\
\hline 31 & 2916.4 & 1458,7 & 2899,3 & 2898.4 & A & 1019.5 & 510,3 & 1002,5 & 1001,5 & 9 \\
\hline 32 & 3031,4 & 1516.2 & 3014,4 & 3013.4 & D & 948.5 & 474,7 & 931,5 & 930,5 & 8 \\
\hline 33 & 3159,5 & 1580.2 & 3142,5 & 3141,5 & K & 833.5 & 417,2 & 816,4 & $\mathbf{8 1 5 , 4}$ & 7 \\
\hline 34 & 3256.5 & 1628,8 & 3239,5 & 3238.5 & $\mathbf{P}$ & 705.4 & 353,2 & 688,3 & 687,4 & 6 \\
\hline 35 & 3369,6 & 1685.3 & 3352,6 & 3351,6 & L & 608,3 & & 591,3 & 590,3 & 5 \\
\hline 36 & 3456,7 & 1728.8 & 3439,6 & 3438,6 & S & 495,2 & & 478,2 & 477,2 & 4 \\
\hline 37 & 3570,7 & 1785.9 & 3553.7 & 3552,7 & $\mathrm{~N}$ & 408,2 & & 391,2 & & 3 \\
\hline 38 & 3717,7 & 1859.4 & 3700,7 & 3699,7 & $M+16$ & 294,1 & & 277,1 & & 2 \\
\hline 39 & 3863,8 & 1932.4 & 3846,8 & 3845,8 & K & 147,1 & & 130,1 & & 1 \\
\hline
\end{tabular}




\section{MS/MS spectrum}

Note: Base peak (m/z 1202.6) is y37+3H (PPETSASVAATPPPpSTASAPAAVNSSASADKPLSNMK, theoretical m/z of 1201.9) and is not unexpected since fragments with $\mathrm{N}$-terminal proline are typically very intense. It was not identified by the visualisation software (Scaffold) because it does not handle triply-charged fragments. Simarly, the unidentified fragment at $\mathrm{m} / \mathrm{z} 1169$ is $y 36+3 \mathrm{H}$, another fragment with an $\mathrm{N}$-terminal proline.

\section{Full-range}

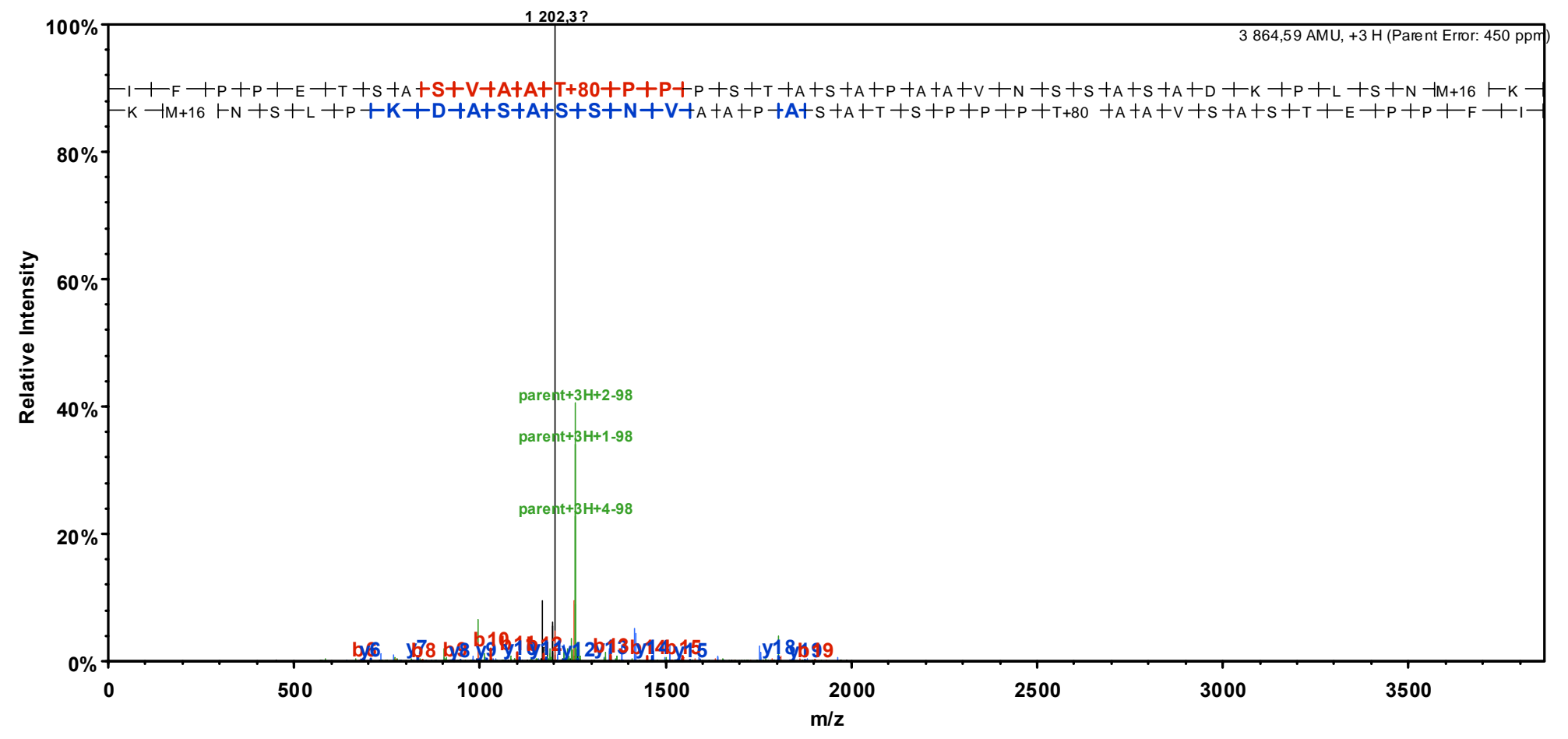

\section{$\underline{\text { Close to precursor }}$}

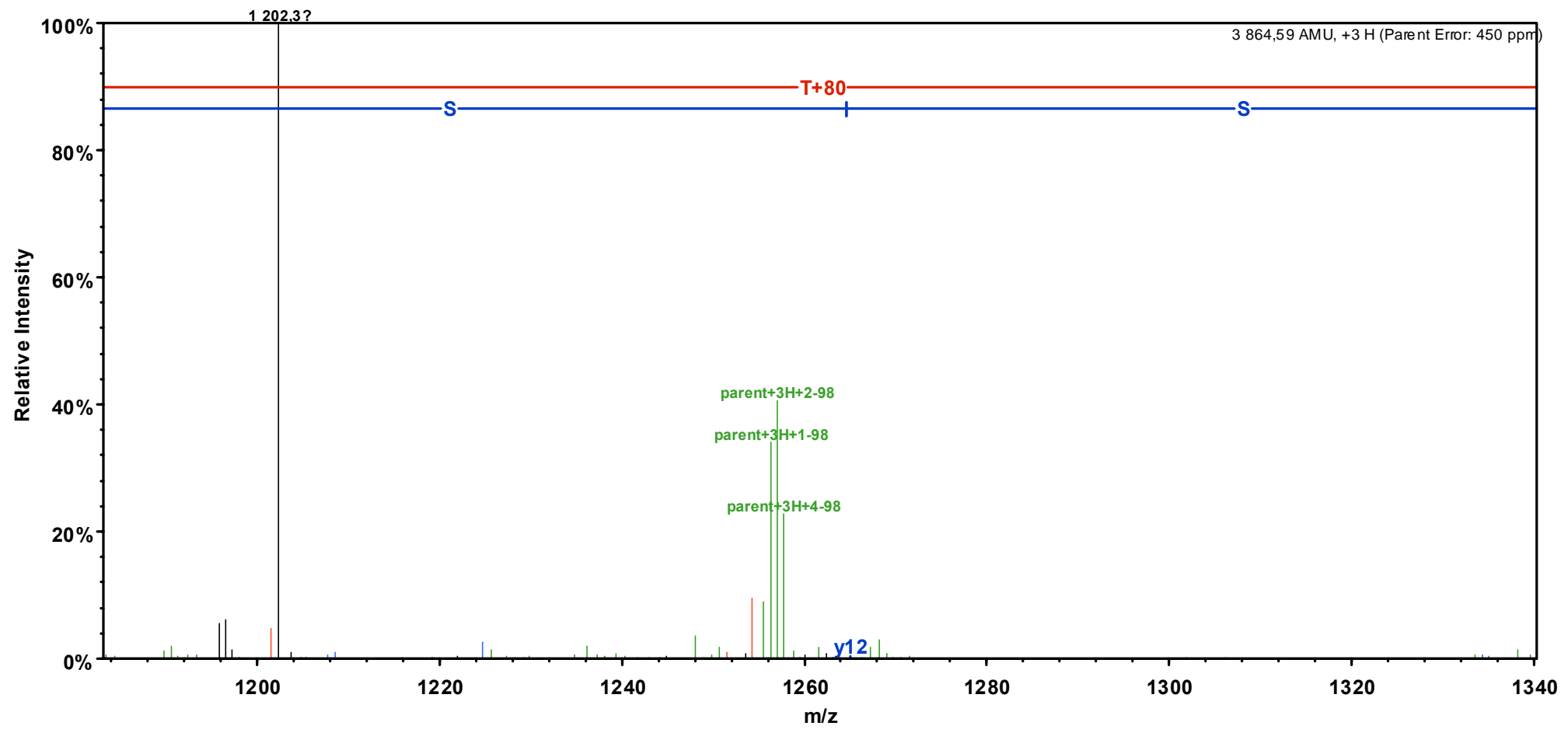




\section{Left of precursor}

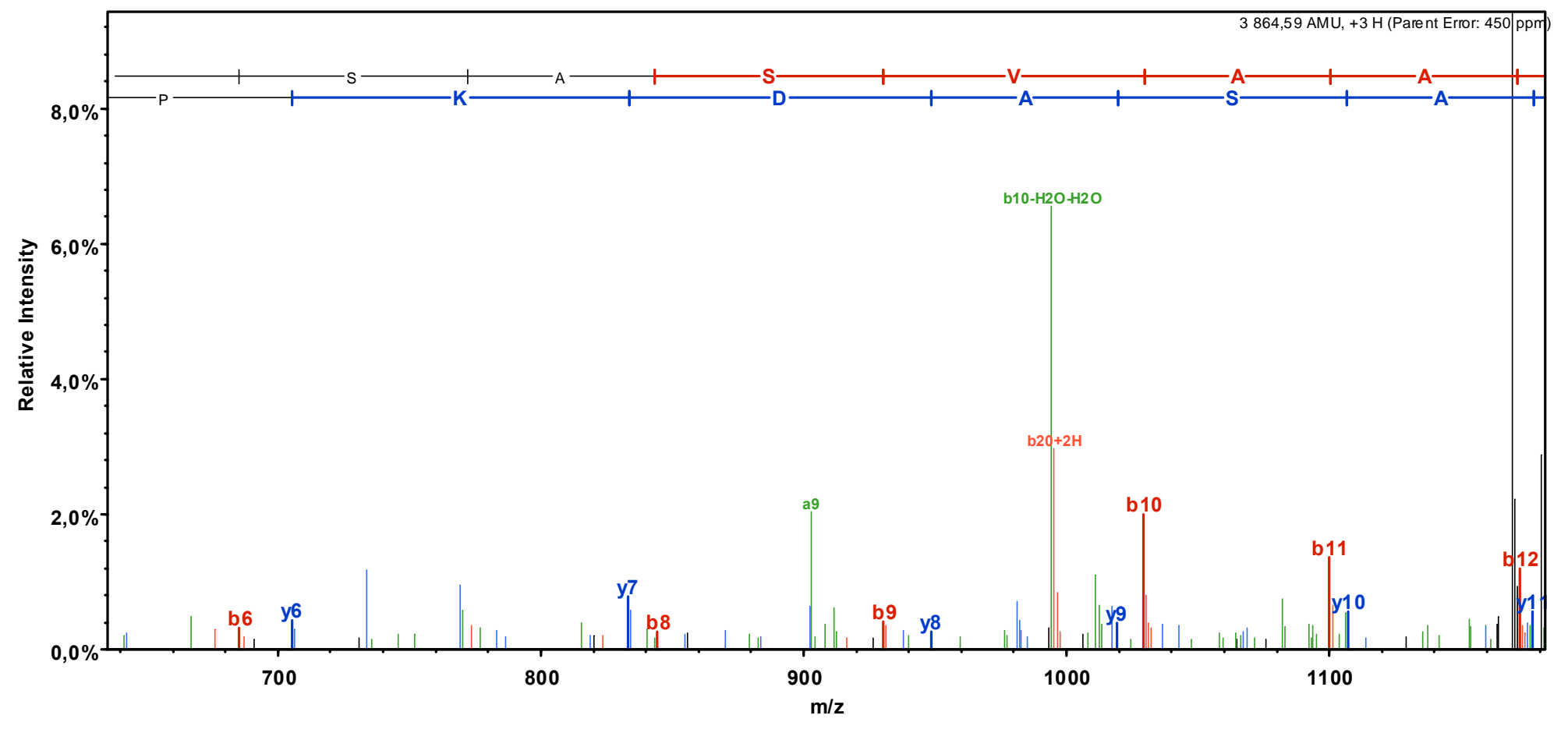

\section{$\underline{\text { Right of precursor }}$}

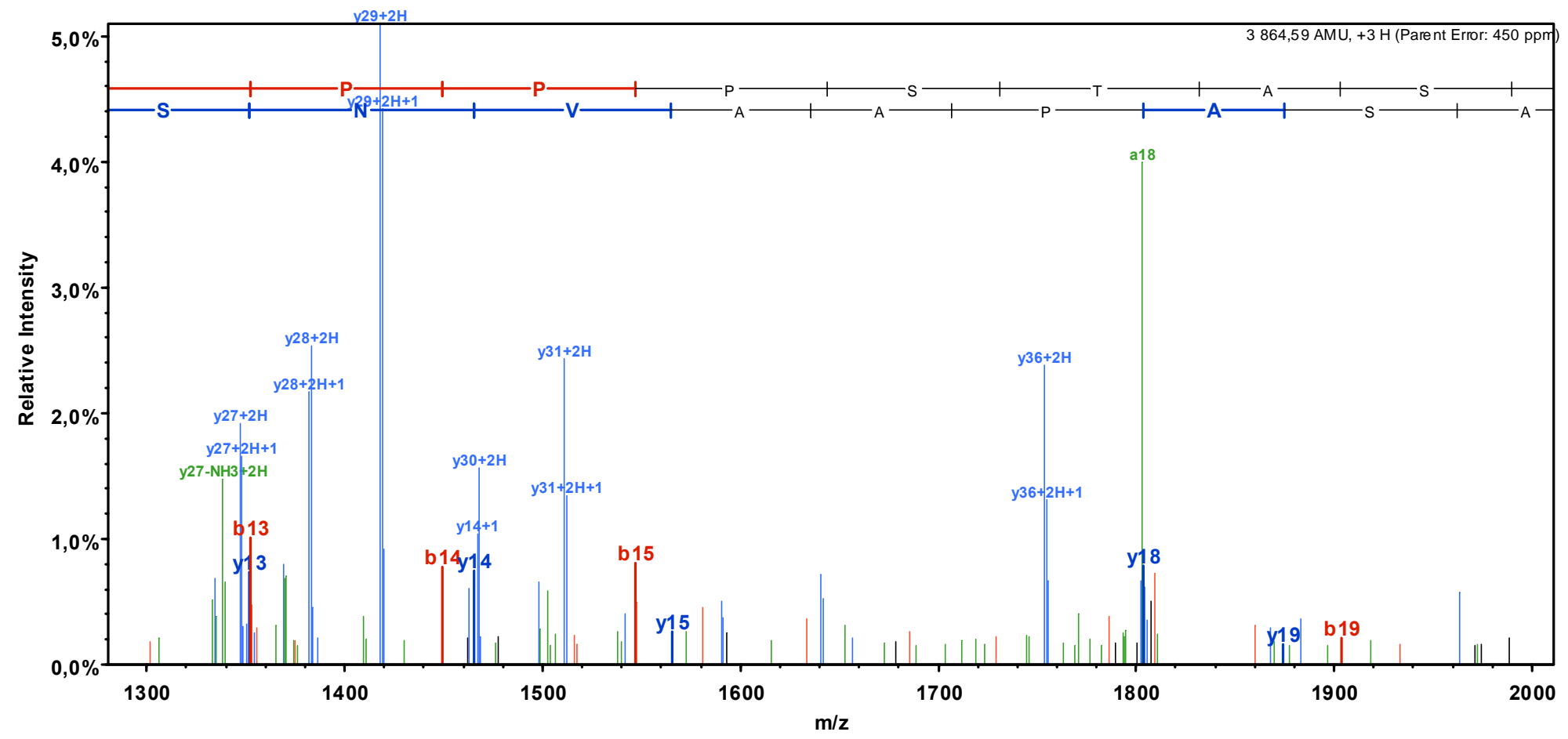




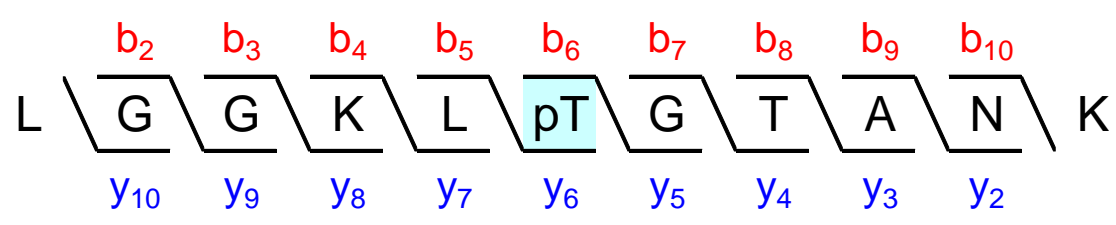

Sequest: $X$ Corr $=3.62, \mathrm{dCn} 1=0.16$ (between $1^{\text {st }}$ and $2^{\text {nd }}$ best matches), $\mathrm{dCn} 2=0.54$ (between $2^{\text {nd }}$ and $3^{\text {rd }}$ best matches). $\mathrm{A}_{\text {score }}=14.42$.

Sequest's second best match with XCorr $=3.04$ is to the same peptide sequence but with an alternative phosphorylation site: LGGKLTGPTANK.

The very weak Ascore indicates that, given the observed MS/MS spectrum, the phosphopeptide identified could either be Sequest's first or second best match (Nat Biotechnol. 2006;24(10):1285-92).

\section{MS/MS spectrum's fragmentation table}

\begin{tabular}{|c|c|c|c|c|c|c|c|c|c|c|}
\hline B & B Ions & $\mathrm{B}+2 \mathrm{H}$ & $\mathrm{B}-\mathrm{NH} 3$ & $\mathrm{~B}-\mathrm{H} 2 \mathrm{O}$ & AA & $Y$ Ions & $\mathrm{Y}+2 \mathrm{H}$ & $\mathrm{Y}-\mathrm{NH} 3$ & $\mathrm{Y}-\mathrm{H} 2 \mathrm{O}$ & $Y$ \\
\hline 1 & 114,1 & & & & $\mathrm{~L}$ & 1139.6 & 570,3 & 1122,6 & 1121,6 & 11 \\
\hline 2 & 171.1 & & & & G & 1026.5 & 513.8 & 1009.5 & 1008.5 & 10 \\
\hline 3 & 228.1 & & & & G & 969.5 & 485.2 & 952.5 & 951,5 & 9 \\
\hline 4 & 356.2 & 178,6 & 339,2 & & K & 912.5 & 456.7 & 895,4 & 894,4 & 8 \\
\hline 5 & 469.3 & 235,2 & 452,3 & & L & 784.4 & 392.7 & 767.3 & 766.4 & 7 \\
\hline 6 & 650.3 & 325,7 & 633,3 & 632,3 & $\mathbf{T}+\mathbf{8 0}$ & 671.3 & 336.1 & 654,3 & 653.3 & 6 \\
\hline 7 & 707.3 & 354.2 & 690,3 & 689.3 & G & 490.3 & & 473.2 & 472,3 & 5 \\
\hline 8 & 808.4 & 404,7 & 791.4 & 790.4 & $\mathbf{T}$ & 433.2 & & 416.2 & 415.2 & 4 \\
\hline 9 & 879.4 & 440.2 & 862.4 & 861,4 & A & 332,2 & & 315.2 & & 3 \\
\hline 10 & 993.5 & 497.2 & 976.5 & 975.5 & $\mathrm{~N}$ & 261.2 & & 244.1 & & 2 \\
\hline 11 & 1139,6 & 570,3 & 1122,6 & 1121,6 & K & 147,1 & & 130,1 & & 1 \\
\hline
\end{tabular}




\section{MS/MS spectrum}

\section{Full-range}

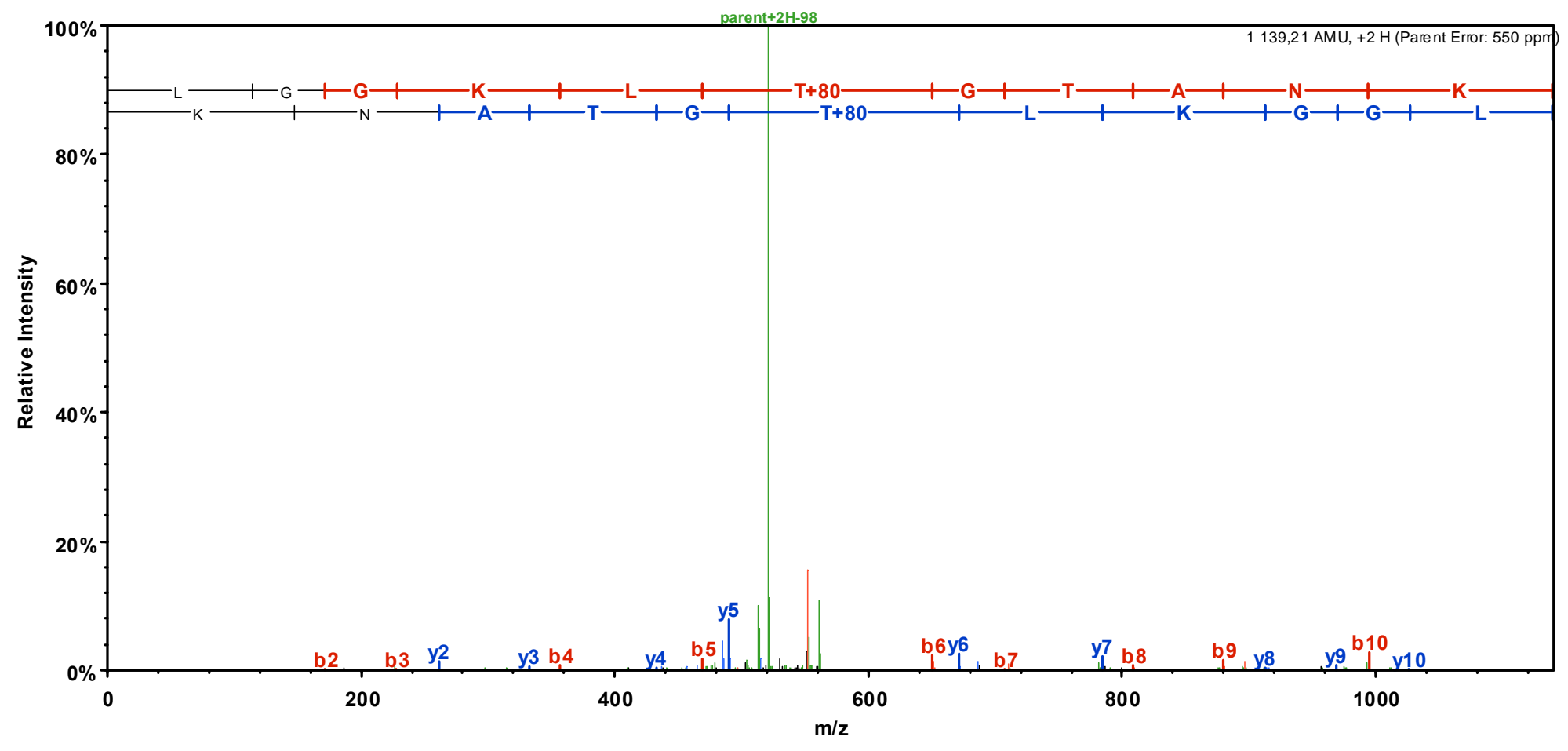

\section{Close to precursor}

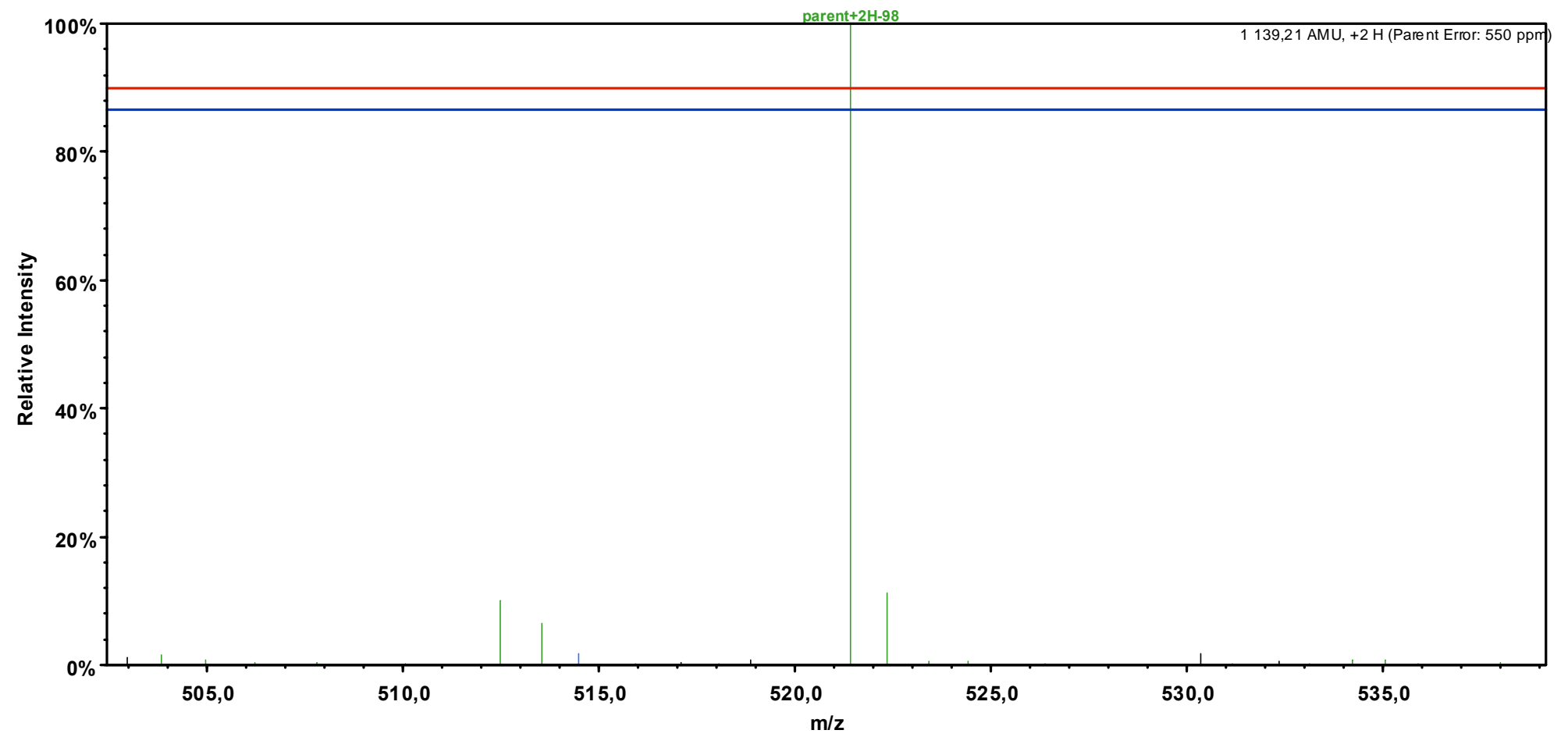




\section{Left of precursor}

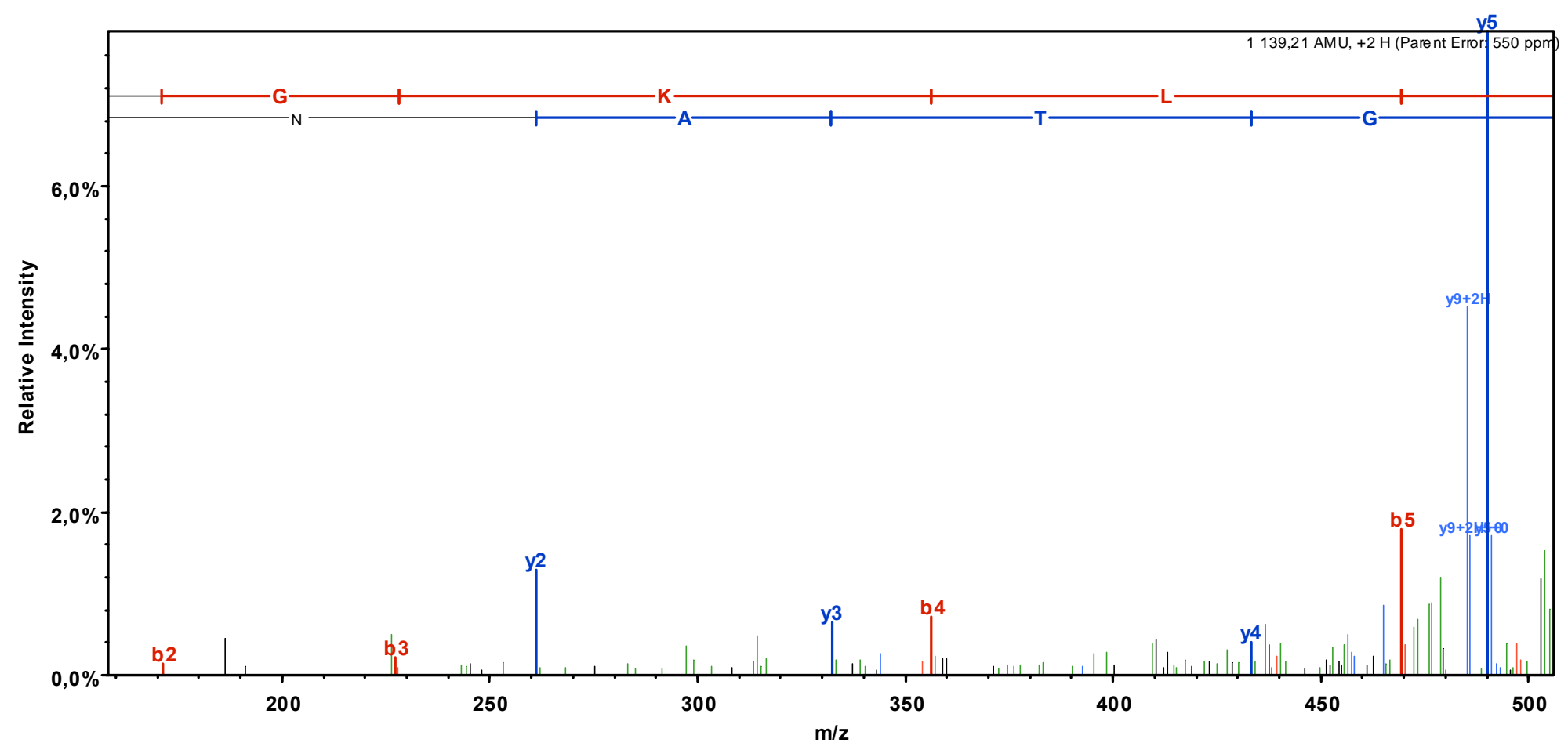

\section{$\underline{\text { Right of precursor }}$}

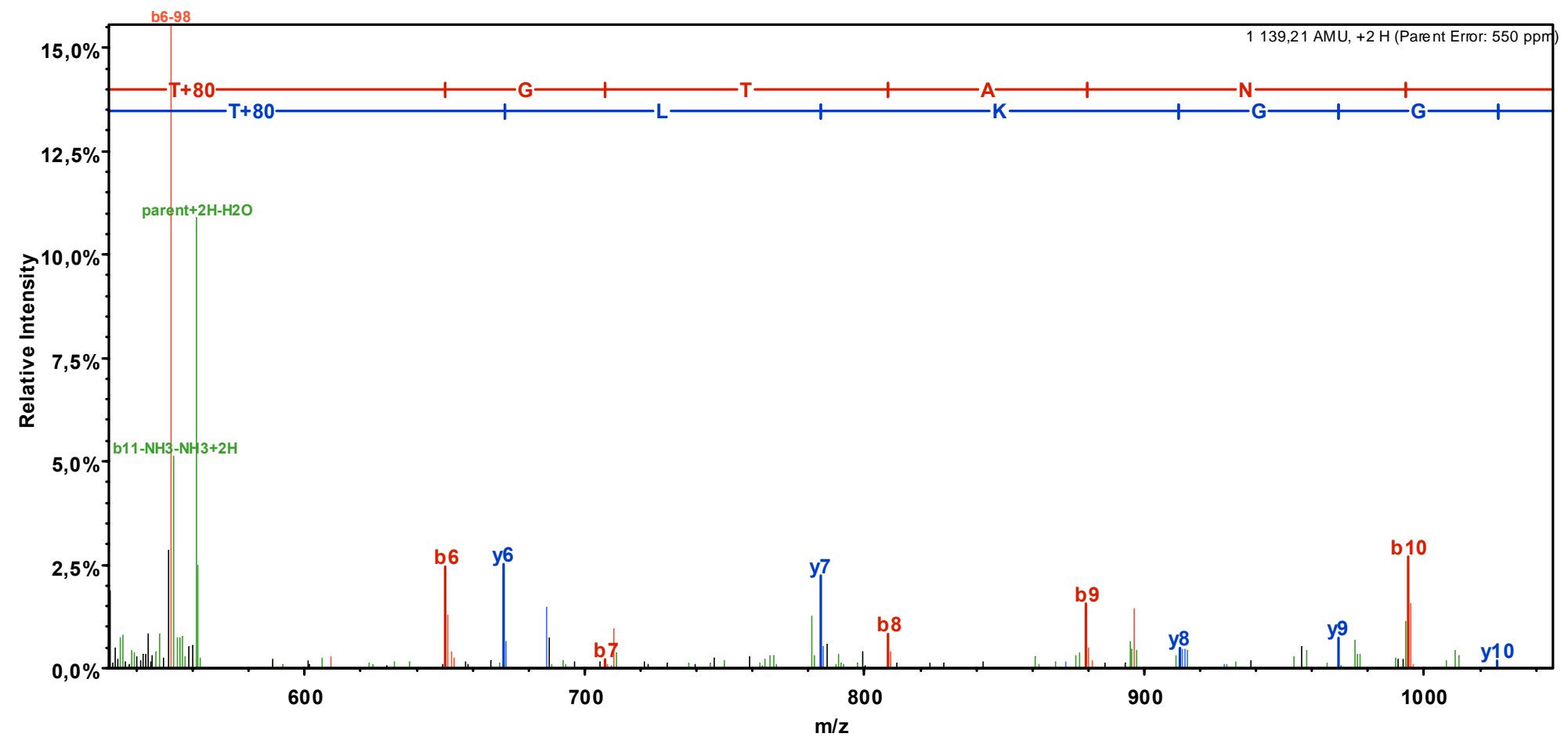




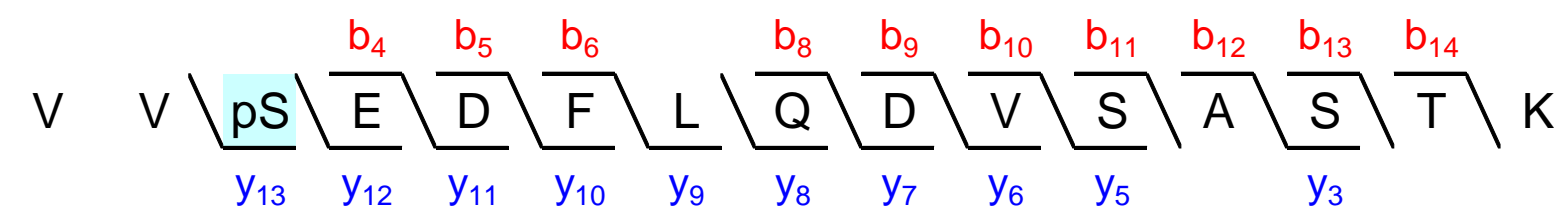

Sequest: XCorr $=3.96, \mathrm{dCn}=0.71$.

Ascore $=53.75$

Phosphorylation site is localized with $\geq 99 \%$ certainty as Ascore $\geq 20$ (Nat Biotechnol. 2006;24(10):1285-92).

\section{MS/MS spectrum's fragmentation table}

\begin{tabular}{|c|c|c|c|c|c|c|c|c|c|c|}
\hline B & B Ions & $\mathrm{B}+2 \mathrm{H}$ & $\mathrm{B}-\mathrm{NH} 3$ & $\mathrm{~B}-\mathrm{H} 2 \mathrm{O}$ & AA & $Y$ Ions & $\mathrm{Y}+2 \mathrm{H}$ & $\mathrm{Y}-\mathrm{NH} 3$ & $\mathrm{Y}-\mathrm{H} 2 \mathrm{O}$ & $Y$ \\
\hline 1 & 100,1 & & & & $\mathrm{~V}$ & 1704,8 & 852,9 & 1687,7 & 1686.8 & 15 \\
\hline 2 & 199,1 & & & & V & 1605,7 & 803,4 & 1588,7 & 1587,7 & 14 \\
\hline 3 & 366.1 & & & 348,1 & $S+80$ & 1506.6 & 753.8 & 1489,6 & 1488,6 & 13 \\
\hline 4 & 495.2 & & & 477,2 & E & 1339,6 & 670,3 & 1322.6 & 1321,6 & 12 \\
\hline 5 & 610.2 & & & 592,2 & D & 1210.6 & 605,8 & 1193,6 & 1192,6 & 11 \\
\hline 6 & 757.3 & 379,1 & & 739.3 & $F$ & 1095.6 & 548,3 & 1078.5 & 1077,6 & 10 \\
\hline 7 & 870,4 & 435,7 & & 852,4 & L & 948.5 & 474.8 & 931,5 & 930,5 & 9 \\
\hline 8 & 998.4 & 499,7 & 981,4 & 980,4 & $\mathbf{Q}$ & $\mathbf{8 3 5 . 4}$ & 418,2 & 818.4 & 817,4 & 8 \\
\hline 9 & 1113.5 & 557,2 & 1096.4 & 1095.4 & D & 707.4 & 354,2 & 690,3 & 689,3 & 7 \\
\hline 10 & 1212.5 & 606,8 & 1195,5 & 1194.5 & $\mathrm{~V}$ & 592.3 & 296,7 & 575,3 & 574,3 & 6 \\
\hline 11 & 1299.6 & 650,3 & 1282,5 & 1281,5 & S & 493.3 & & 476,2 & 475,3 & 5 \\
\hline 12 & 1370.6 & 685,8 & 1353.6 & 1352.6 & A & 406,2 & & 389,2 & 388,2 & 4 \\
\hline 13 & 1457.6 & 729,3 & 1440.6 & 1439,6 & S & 335.2 & & 318,2 & 317,2 & 3 \\
\hline 14 & 1558.7 & 779,8 & 1541,6 & 1540.7 & $\mathbf{T}$ & 248,2 & & 231,1 & 230,2 & 2 \\
\hline 15 & 1704.8 & 852,9 & 1687,7 & 1686.8 & K & 147,1 & & 130,1 & & 1 \\
\hline
\end{tabular}




\section{MS/MS spectrum}

\section{Full-range}

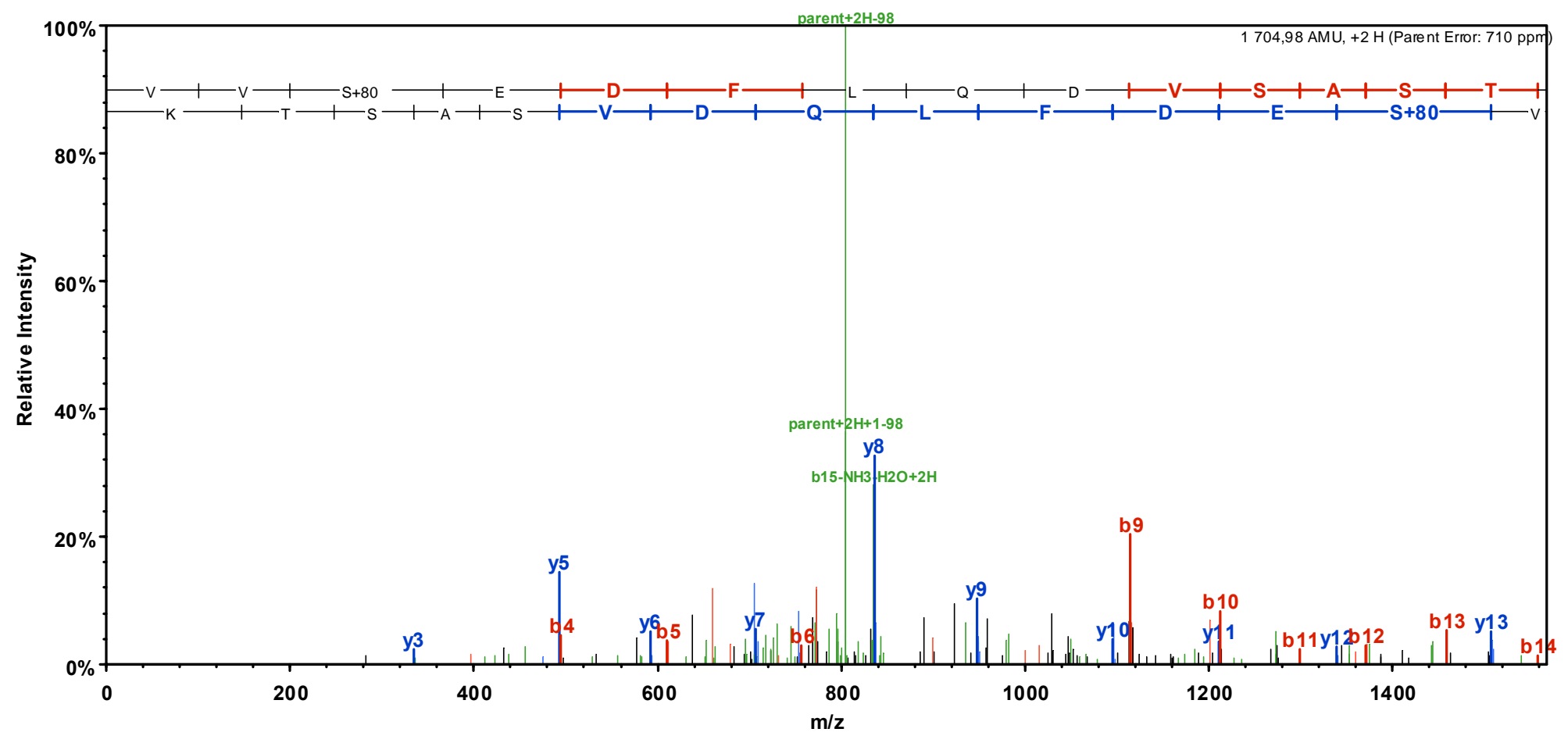

\section{$\underline{\text { Close to precursor }}$}

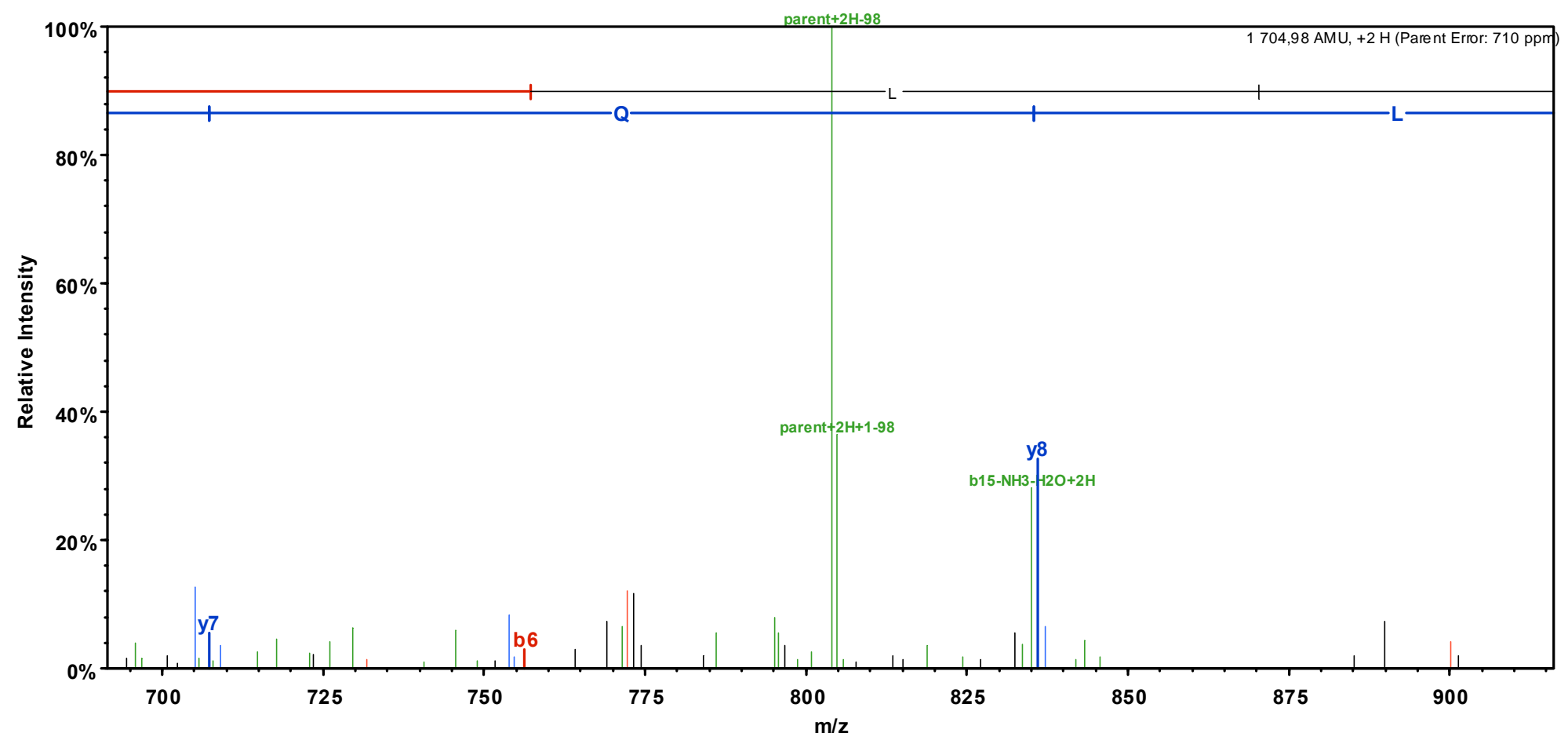




\section{Left of precursor}

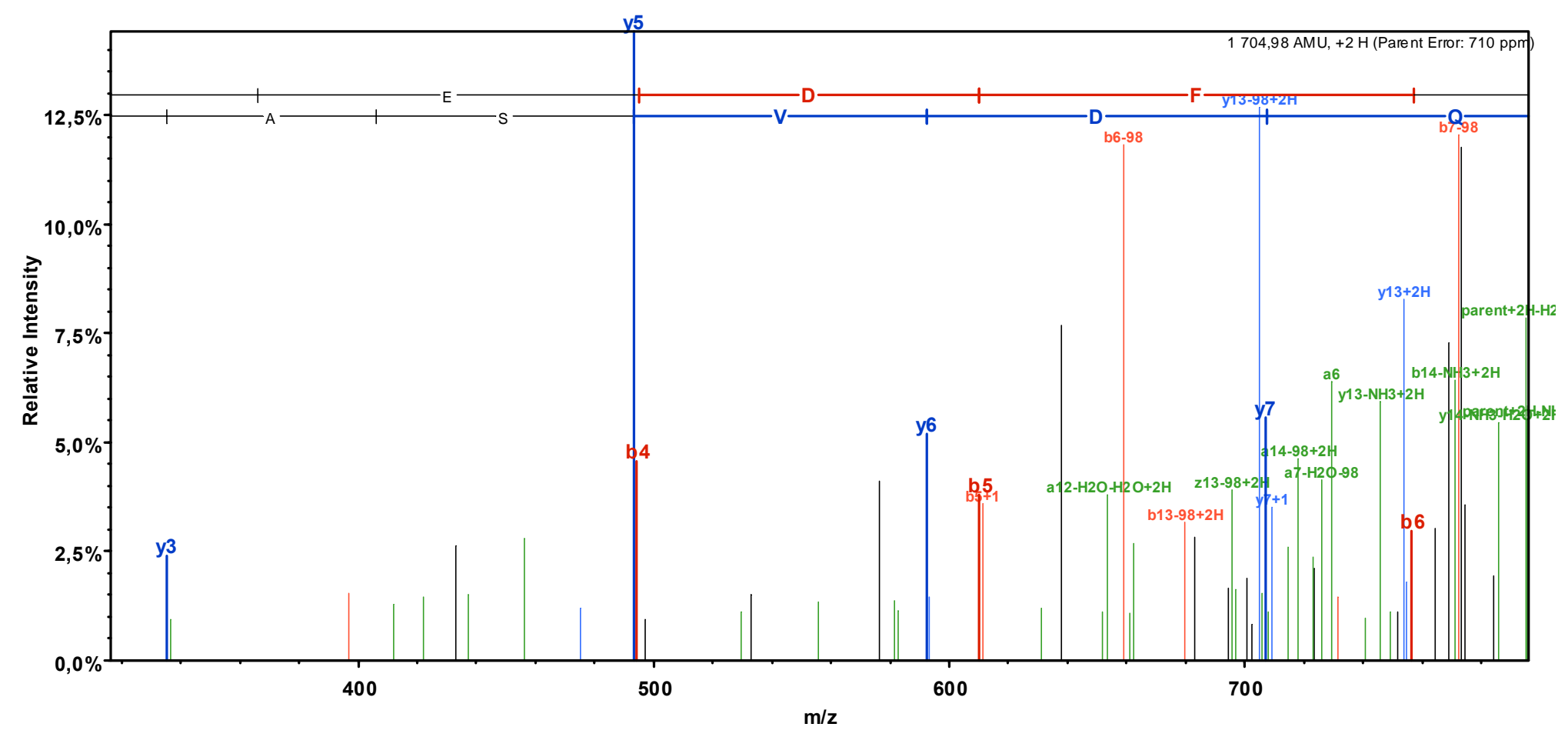

\section{$\underline{\text { Right of precursor }}$}

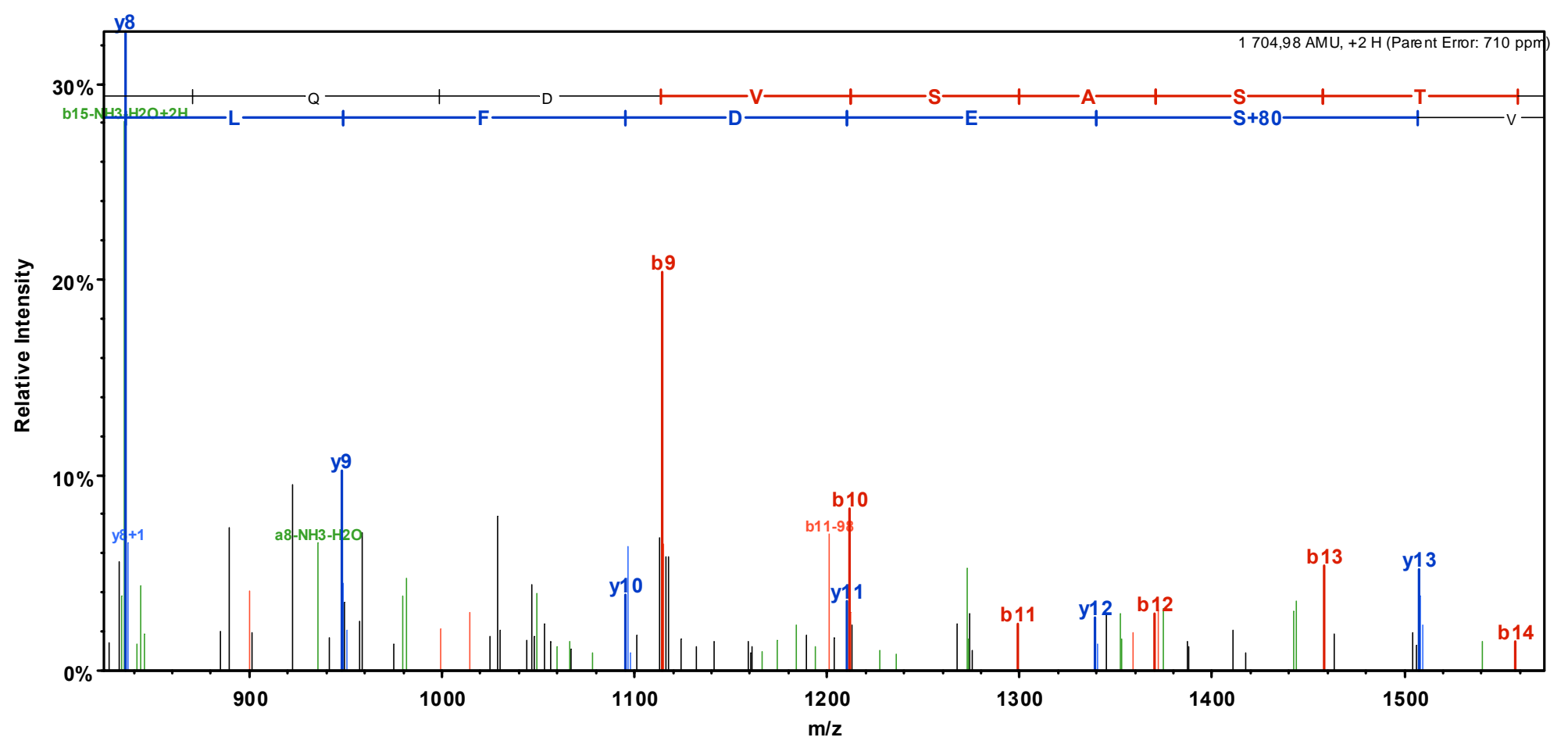




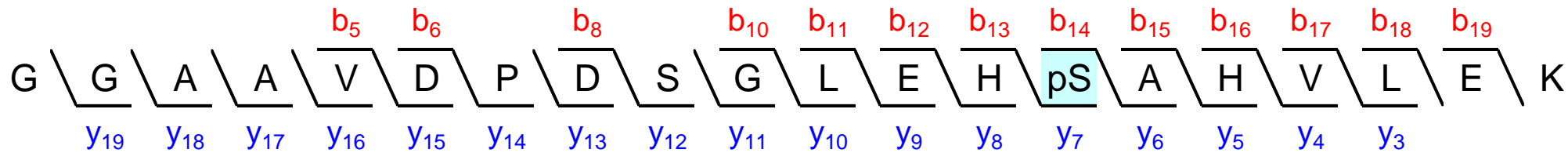

Sequest: XCorr $=3.85, \mathrm{dCn}=0.88$.

Ascore $=21.05$

Phosphorylation site is localized with $\geq 99 \%$ certainty as Ascore $\geq 20$ (Nat Biotechnol. 2006;24(10):1285-92).

\section{MS/MS spectrum's fragmentation table}

\begin{tabular}{|c|c|c|c|c|c|c|c|c|c|c|}
\hline B & B Ions & $\mathrm{B}+2 \mathrm{H}$ & $\mathrm{B}-\mathrm{NH} 3$ & $\mathrm{~B}-\mathrm{H} 2 \mathrm{O}$ & AA & $Y$ Ions & $\mathrm{Y}+2 \mathrm{H}$ & $\mathrm{Y}-\mathrm{NH} 3$ & $\mathrm{Y}-\mathrm{H} 2 \mathrm{O}$ & $Y$ \\
\hline 1 & 58,0 & & & & G & 2068.9 & 1035,0 & 2051,9 & 2050,9 & 20 \\
\hline 2 & 115,1 & & & & G & 2011,9 & 1006.5 & 1994,9 & 1993.9 & 19 \\
\hline 3 & 186,1 & & & & A & 1954,9 & 977.9 & 1937,9 & 1936,9 & 18 \\
\hline 4 & 257,1 & & & & A & 1883,9 & 942,4 & 1866,8 & 1865,8 & 17 \\
\hline 5 & 356.2 & & & & V & 1812,8 & 906.9 & 1795,8 & 1794,8 & 16 \\
\hline 6 & 471.2 & 236.1 & & 453.2 & D & 1713.7 & 857.4 & 1696.7 & 1695.7 & 15 \\
\hline 7 & 568,3 & 284,6 & & 550,3 & $\mathbf{P}$ & 1598.7 & 799,9 & 1581,7 & 1580.7 & 14 \\
\hline 8 & 683.3 & 342,2 & & 665.3 & D & 1501.7 & 751.3 & 1484,6 & 1483.7 & 13 \\
\hline 9 & 770,3 & 385,7 & & 752,3 & S & 1386.6 & 693.8 & 1369.6 & 1368.6 & 12 \\
\hline 10 & 827,4 & 414,2 & & 809,3 & G & 1299.6 & 650.3 & 1282.6 & 1281,6 & 11 \\
\hline 11 & 940.4 & 470,7 & & 922.4 & L & 1242.6 & 621,8 & 1225.6 & 1224,6 & 10 \\
\hline 12 & 1069.5 & 535,2 & & 1051,5 & $E$ & 1129.5 & 565,3 & 1112.5 & 1111,5 & 9 \\
\hline 13 & 1206.5 & 603,8 & & 1188.5 & H & 1000.5 & 500.7 & 983.4 & 982,5 & 8 \\
\hline 14 & 1373.5 & 687,3 & & 1355.5 & $S+80$ & 863.4 & 432,2 & 846,4 & 845.4 & 7 \\
\hline 15 & 1444,6 & 722,8 & & 1426.6 & A & 696.4 & 348,7 & 679.4 & 678,4 & 6 \\
\hline 16 & 1581.6 & 791.3 & & 1563.6 & $\mathbf{H}$ & 625.4 & 313,2 & 608,3 & 607.4 & 5 \\
\hline 17 & 1680.7 & 840.9 & & 1662,7 & V & 488.3 & & 471,3 & 470,3 & 4 \\
\hline 18 & 1793.8 & 897.4 & & 1775,8 & L & 389.2 & & 372,2 & 371,2 & 3 \\
\hline 19 & 1922,8 & 961.9 & & 1904,8 & $E$ & 276,2 & & 259,1 & 258.1 & 2 \\
\hline 20 & 2068,9 & 1035,0 & 2051,9 & 2050,9 & K & 147,1 & & 130,1 & & 1 \\
\hline
\end{tabular}




\section{MS/MS spectrum}

\section{Full-range}

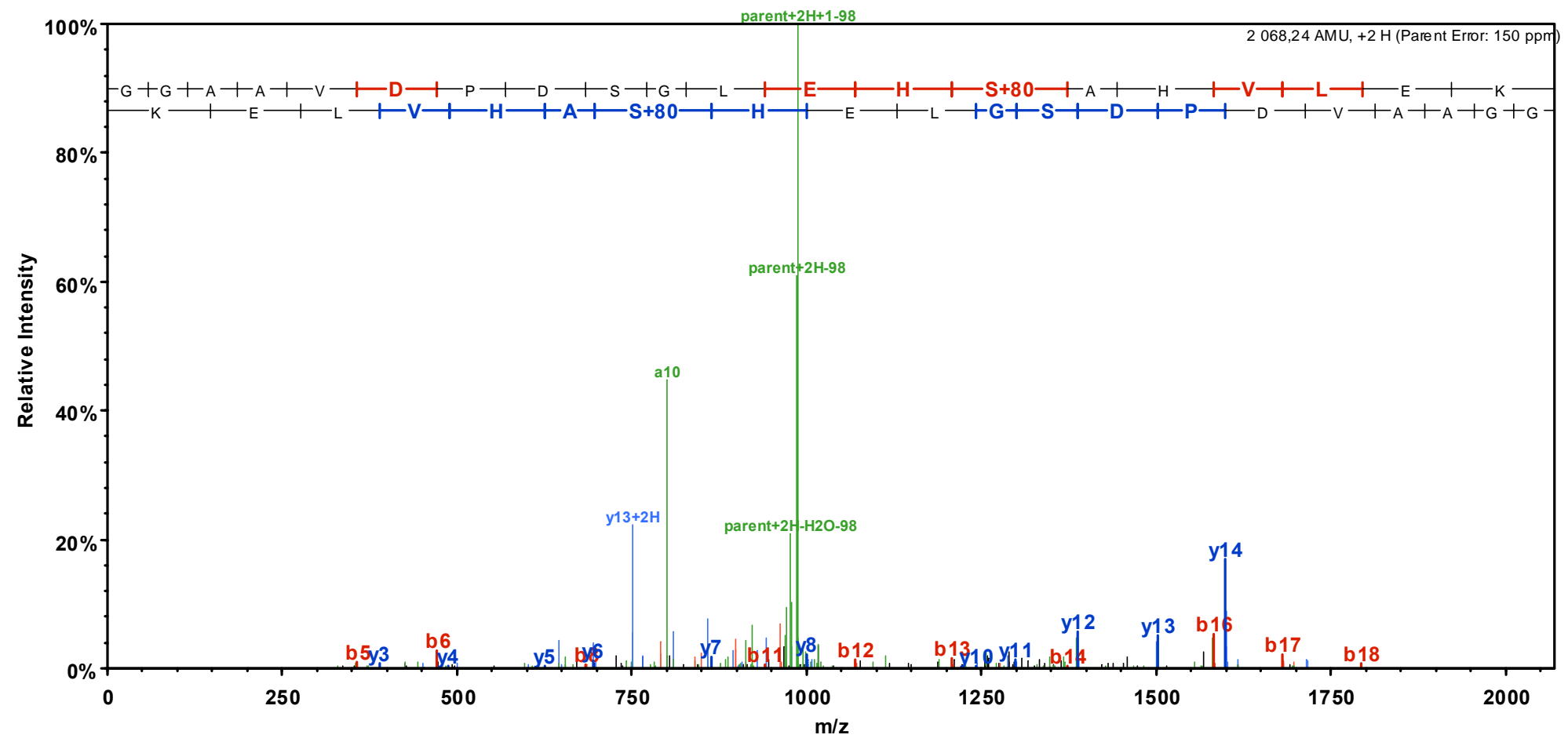

\section{Close to precursor}

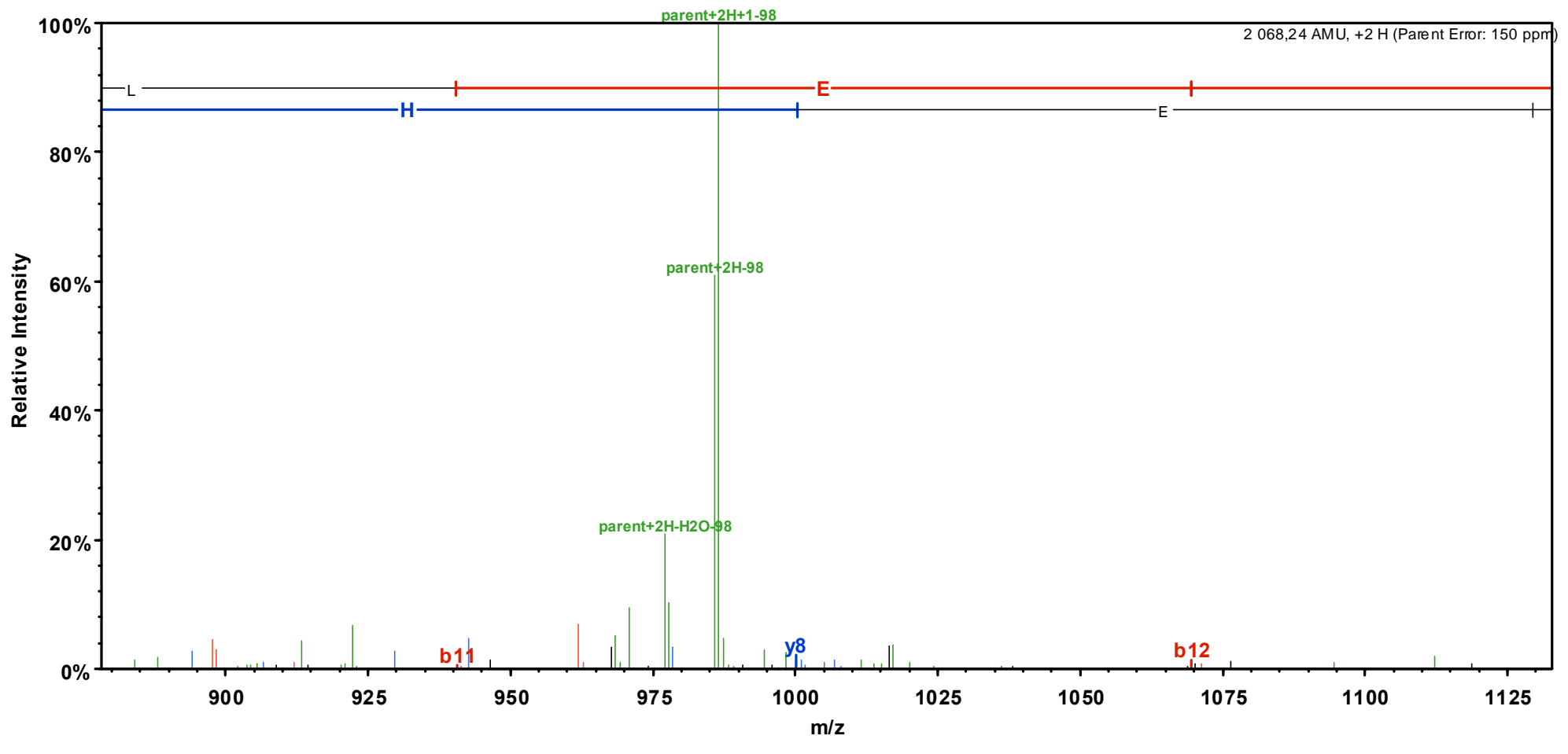




\section{Left of precursor}

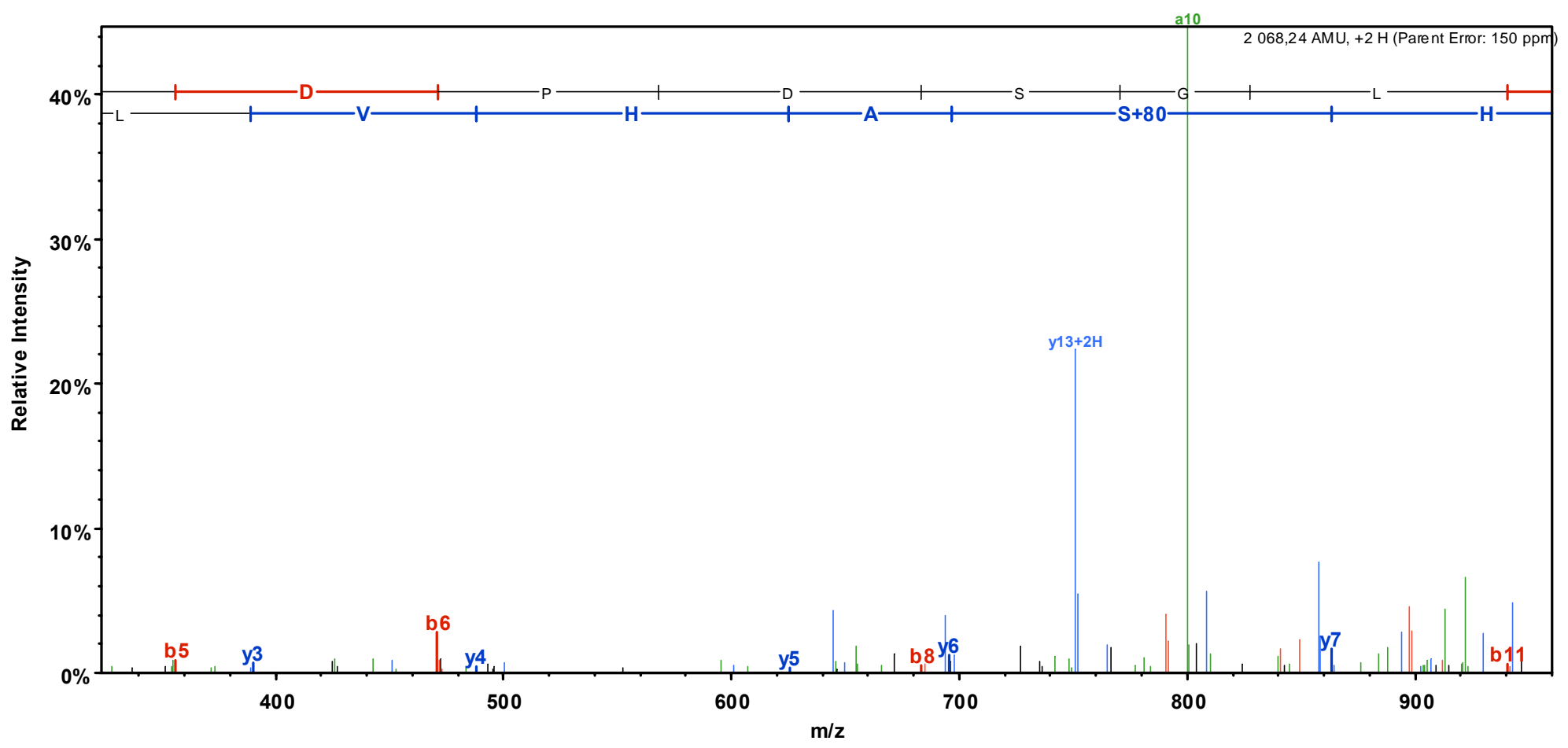

\section{$\underline{\text { Right of precursor }}$}

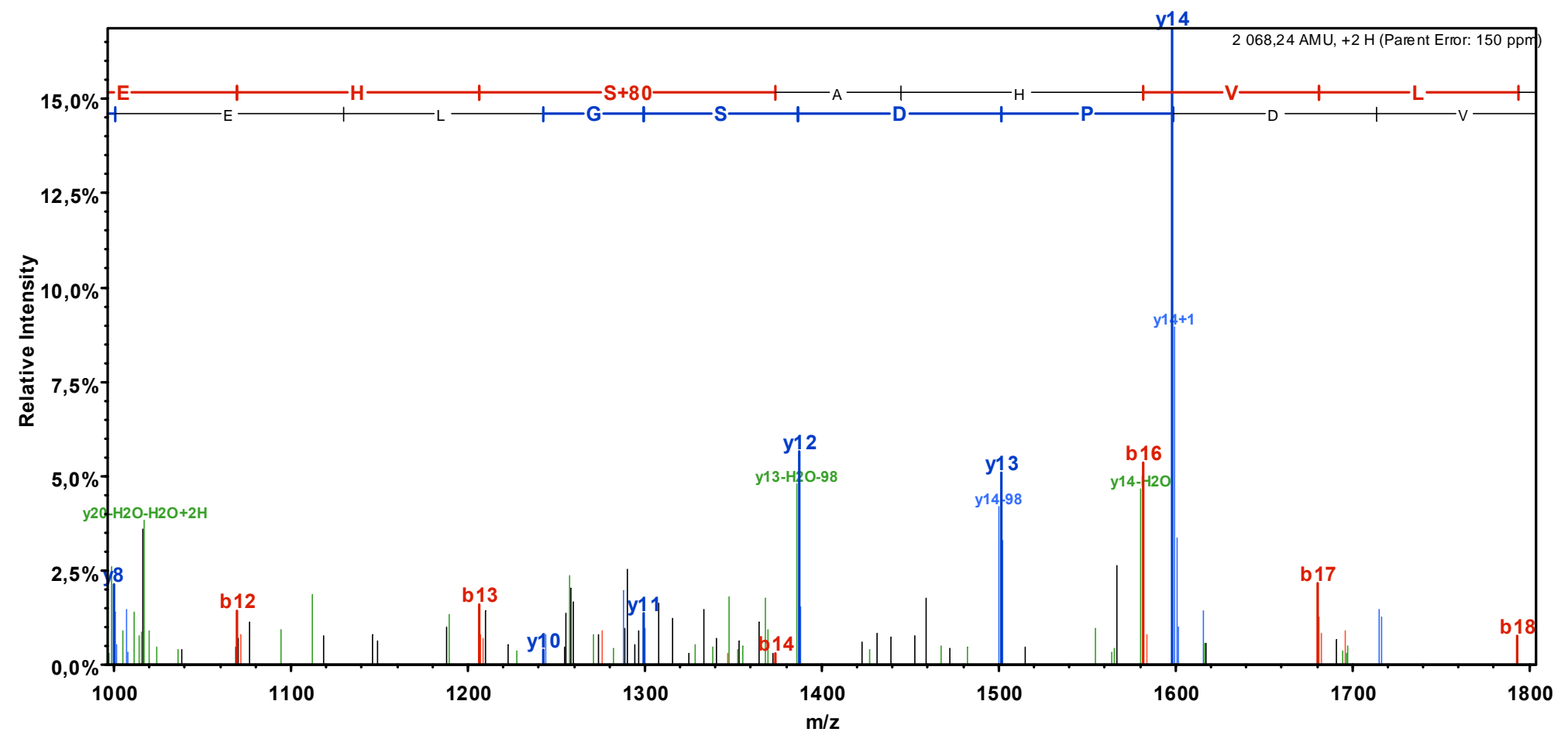




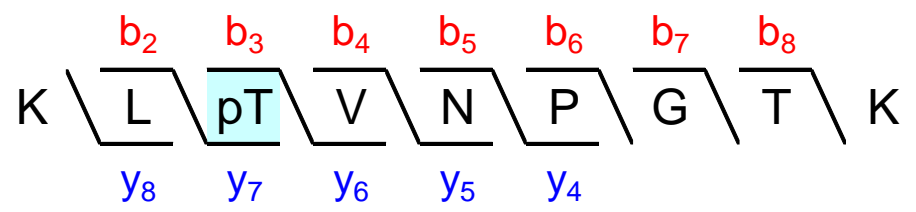

Sequest: XCorr $=1.96, \mathrm{dCn}=0.77$.

Ascore $=22.88$

Phosphorylation site is localized with $\geq 99 \%$ certainty as Ascore $\geq 20$ (Nat Biotechnol. 2006;24(10):1285-92).

MS/MS spectrum's fragmentation table

\begin{tabular}{|c|c|c|c|c|c|c|c|c|c|c|}
\hline B & B Ions & $\mathrm{B}+2 \mathrm{H}$ & $\mathrm{B}-\mathrm{NH} 3$ & $\mathrm{~B}-\mathrm{H} 2 \mathrm{O}$ & AA & $Y$ Ions & $\mathrm{Y}+2 \mathrm{H}$ & $\mathrm{Y}-\mathrm{NH} 3$ & $\mathrm{Y}-\mathrm{H} 2 \mathrm{O}$ & Y \\
\hline 1 & 129,1 & 65.1 & 112,1 & & $\mathrm{~K}$ & 1037.5 & 519,3 & 1020.5 & 1019,5 & 9 \\
\hline 2 & 242.2 & 121,6 & 225.2 & & $\mathbf{L}$ & 909.4 & 455.2 & 892,4 & 891,4 & 8 \\
\hline 3 & 423.2 & 212.1 & 406,2 & 405,2 & $\mathbf{T}+\mathbf{8 0}$ & 796.4 & 398.7 & 779.3 & 778.4 & 7 \\
\hline 4 & 522,3 & 261.6 & 505,2 & 504.3 & V & 615.3 & 308.2 & 598.3 & 597,3 & 6 \\
\hline 5 & 636.3 & 318.7 & 619.3 & 618,3 & $\mathrm{~N}$ & 516.3 & & 499.3 & 498.3 & 5 \\
\hline 6 & 733.4 & 367,2 & 716.3 & 715,4 & $\mathbf{P}$ & 402.2 & & 385.2 & 384,2 & 4 \\
\hline 7 & 790.4 & 395,7 & 773.4 & 772,4 & G & 305,2 & & 288,2 & 287,2 & 3 \\
\hline 8 & 891.4 & 446,2 & 874.4 & 873.4 & $\mathbf{T}$ & 248,2 & & 231,1 & 230,2 & 2 \\
\hline 9 & 1037,5 & 519,3 & 1020,5 & 1019,5 & K & 147,1 & & 130,1 & & 1 \\
\hline
\end{tabular}




\section{MS/MS spectrum}

\section{Full-range}

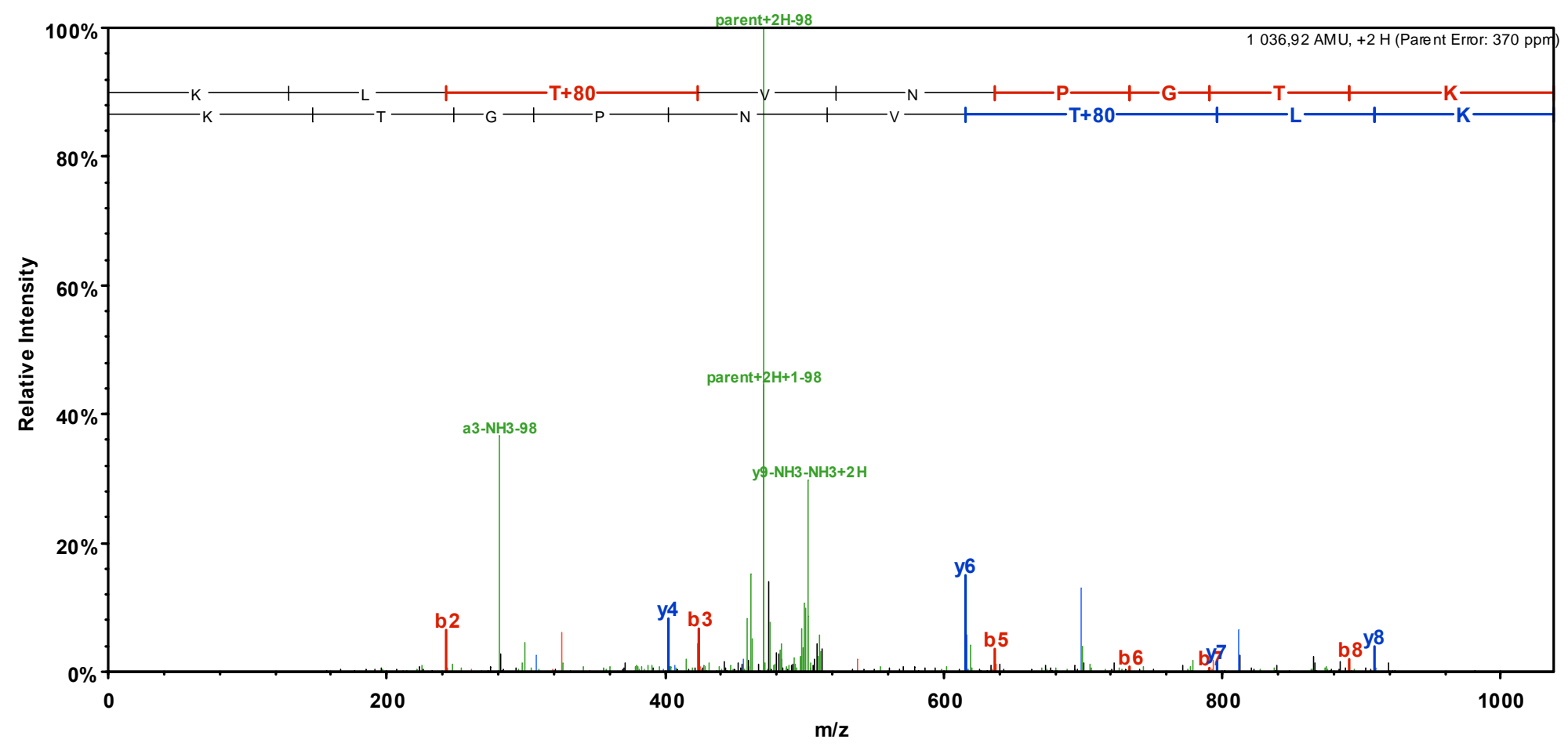

\section{Close to precursor}

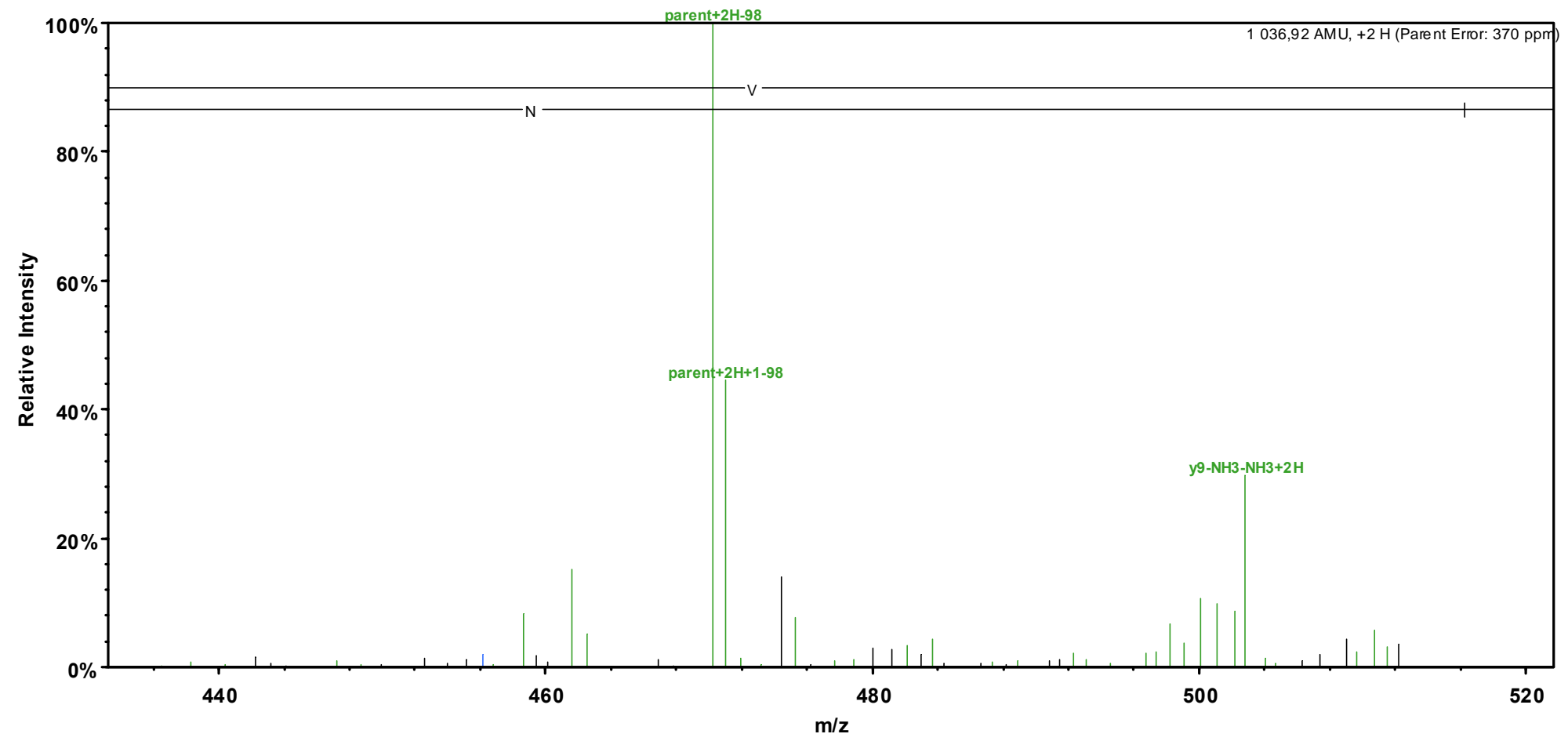




\section{Left of precursor}

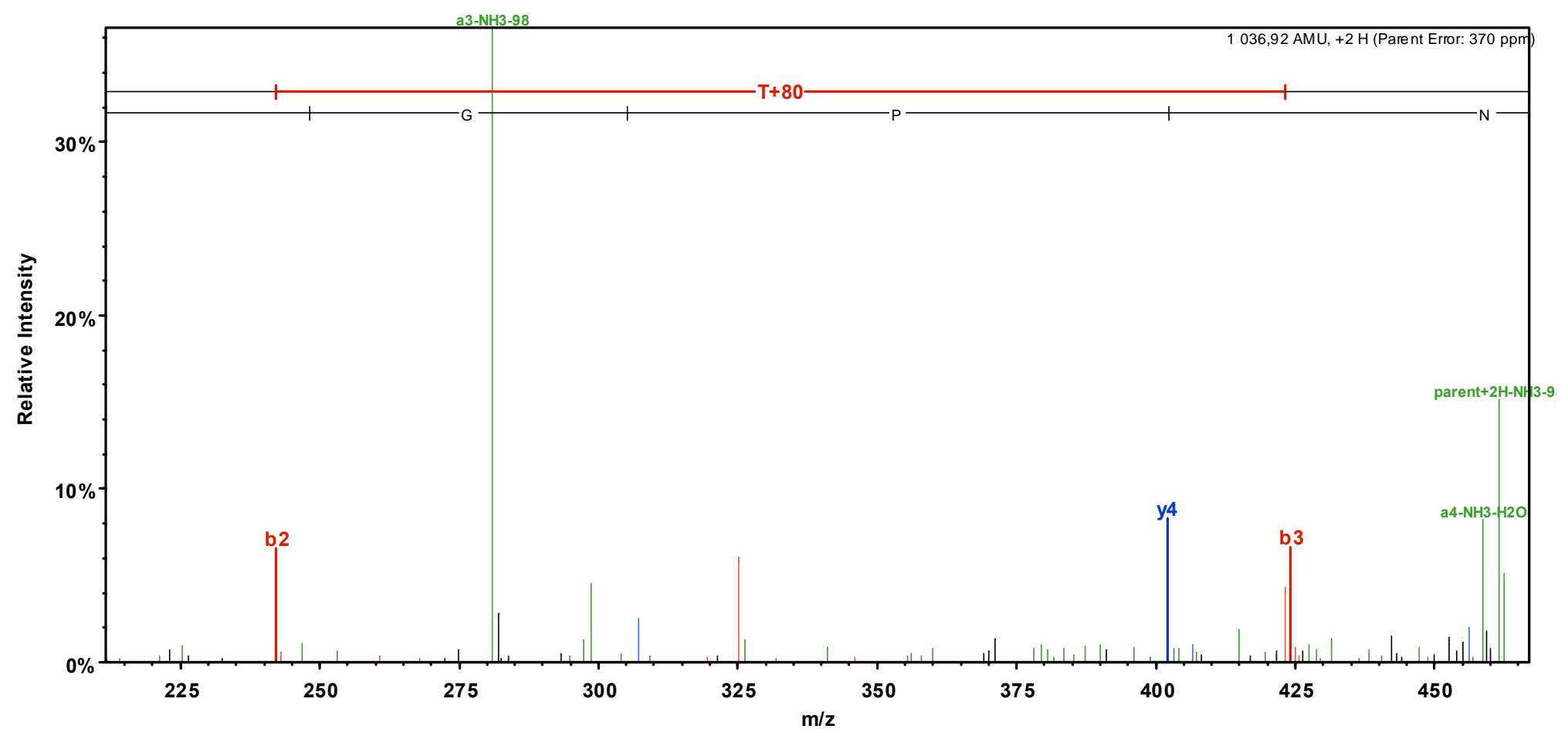

\section{$\underline{\text { Right of precursor }}$}

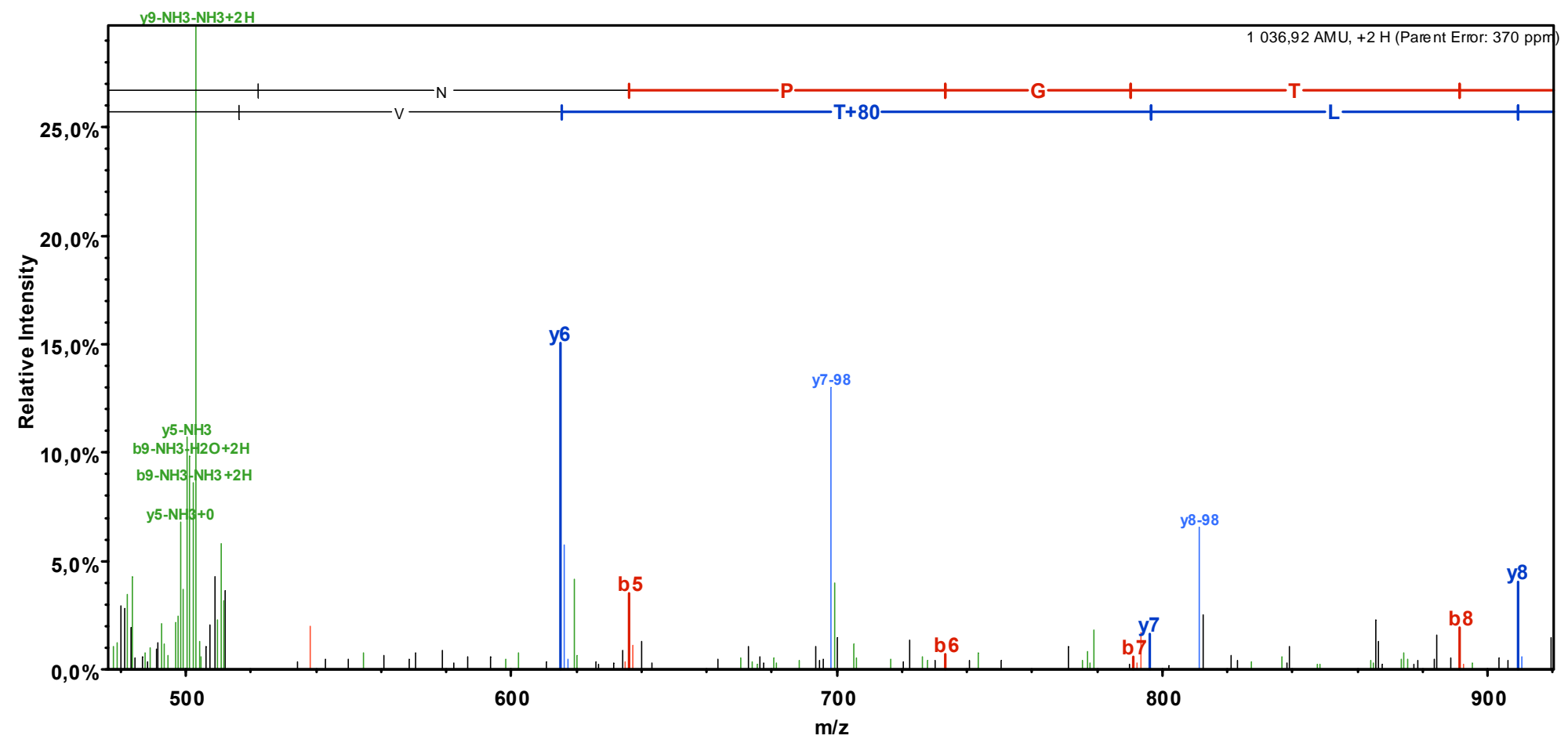




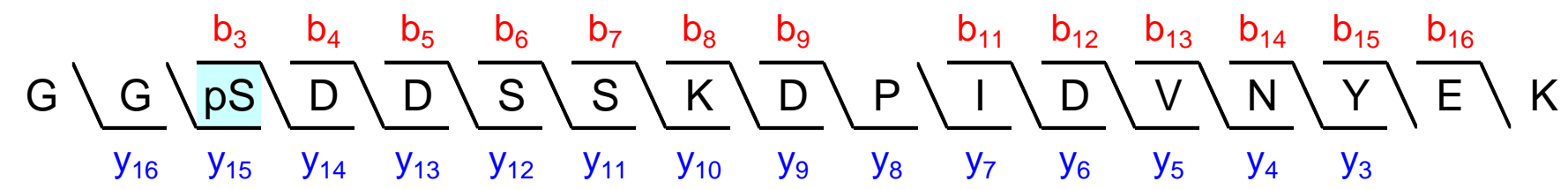

Sequest: XCorr $=5.78, \mathrm{dCn}=0.10$.

Ascore $=50.89$

Phosphorylation site is localized with $\geq 99 \%$ certainty as Ascore $\geq 20$ (Nat Biotechnol. 2006;24(10):1285-92).

\section{MS/MS spectrum's fragmentation table}

\begin{tabular}{|c|c|c|c|c|c|c|c|c|c|c|}
\hline B & $B$ Ions & $\mathrm{B}+2 \mathrm{H}$ & $\mathrm{B}-\mathrm{NH} 3$ & $\mathrm{~B}-\mathrm{H} 2 \mathrm{O}$ & AA & $Y$ Ions & $\mathrm{Y}+2 \mathrm{H}$ & $\mathrm{Y}-\mathrm{NH} 3$ & $\mathrm{Y}-\mathrm{H} 2 \mathrm{O}$ & $\mathrm{Y}$ \\
\hline 1 & 58,0 & & & & G & 1905,8 & 953.4 & 1888,7 & 1887.8 & 17 \\
\hline 2 & 115,1 & & & & G & 1848,8 & 924.9 & 1831,7 & 1830.7 & 16 \\
\hline 3 & 282.0 & & & 264,0 & S+80 & 1791,7 & 896.4 & 1774,7 & 1773,7 & 15 \\
\hline 4 & 397.1 & & & 379.1 & D & 1624.7 & 812,9 & 1607.7 & 1606.7 & 14 \\
\hline 5 & 512.1 & & & 494,1 & D & 1509.7 & 755.4 & 1492,7 & 1491.7 & 13 \\
\hline 6 & 599.1 & 300,1 & & 581,1 & S & 1394.7 & 697.8 & 1377.7 & 1376.7 & 12 \\
\hline 7 & 686.2 & 343,6 & & 668.2 & S & 1307.6 & 654,3 & 1290,6 & 1289.6 & 11 \\
\hline 8 & 814.3 & 407.6 & 797,2 & 796.3 & K & 1220.6 & 610,8 & 1203.6 & 1202.6 & 10 \\
\hline 9 & 929.3 & 465,1 & 912,3 & 911.3 & D & 1092.5 & 546,8 & 1075.5 & 1074.5 & 9 \\
\hline 10 & 1026,3 & 513,7 & 1009.3 & 1008,3 & $\mathbf{P}$ & 977.5 & 489,3 & 960.5 & 959.5 & 8 \\
\hline 11 & 1139.4 & 570,2 & 1122,4 & 1121.4 & I & 880.4 & 440.7 & 863.4 & 862,4 & 7 \\
\hline 12 & 1254.5 & 627.7 & 1237.4 & 1236.4 & D & 767.4 & 384,2 & 750.3 & 749.3 & 6 \\
\hline 13 & 1353.5 & 677,3 & 1336.5 & 1335.5 & V & 652.3 & & 635.3 & 634.3 & 5 \\
\hline 14 & 1467.6 & 734.3 & 1450.5 & 1449.6 & $\mathrm{~N}$ & 553.3 & & 536.2 & 535,3 & 4 \\
\hline 15 & 1630.6 & 815.8 & 1613.6 & 1612,6 & $\mathbf{Y}$ & 439.2 & & 422,2 & 421.2 & 3 \\
\hline 16 & 1759.7 & 880,3 & 1742,6 & 1741.7 & E & 276,2 & & 259,1 & 258,1 & 2 \\
\hline 17 & 1905,8 & 953.4 & 1888.7 & 1887,8 & K & 147,1 & & 130,1 & & 1 \\
\hline
\end{tabular}




\section{MS/MS spectrum}

\section{Full-range}

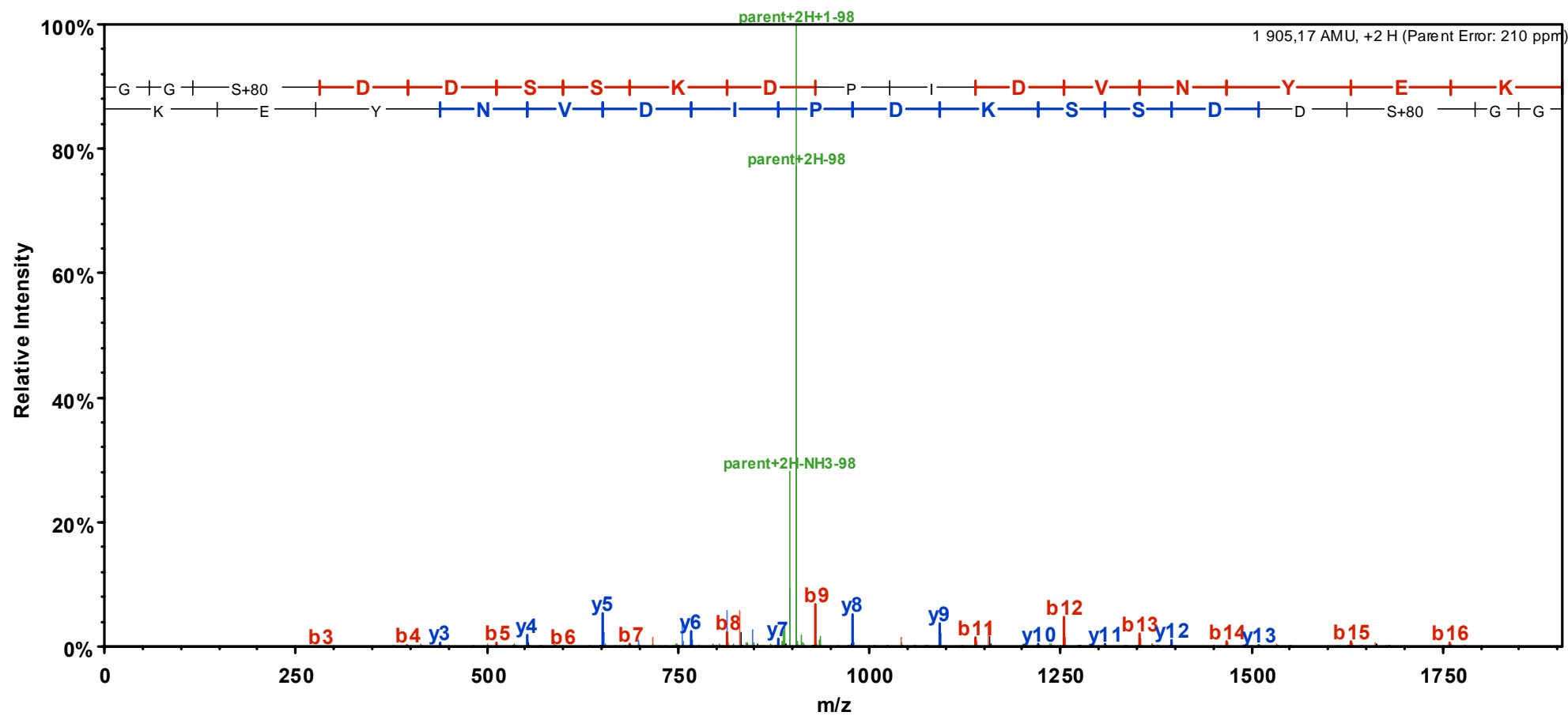

\section{Close to precursor}

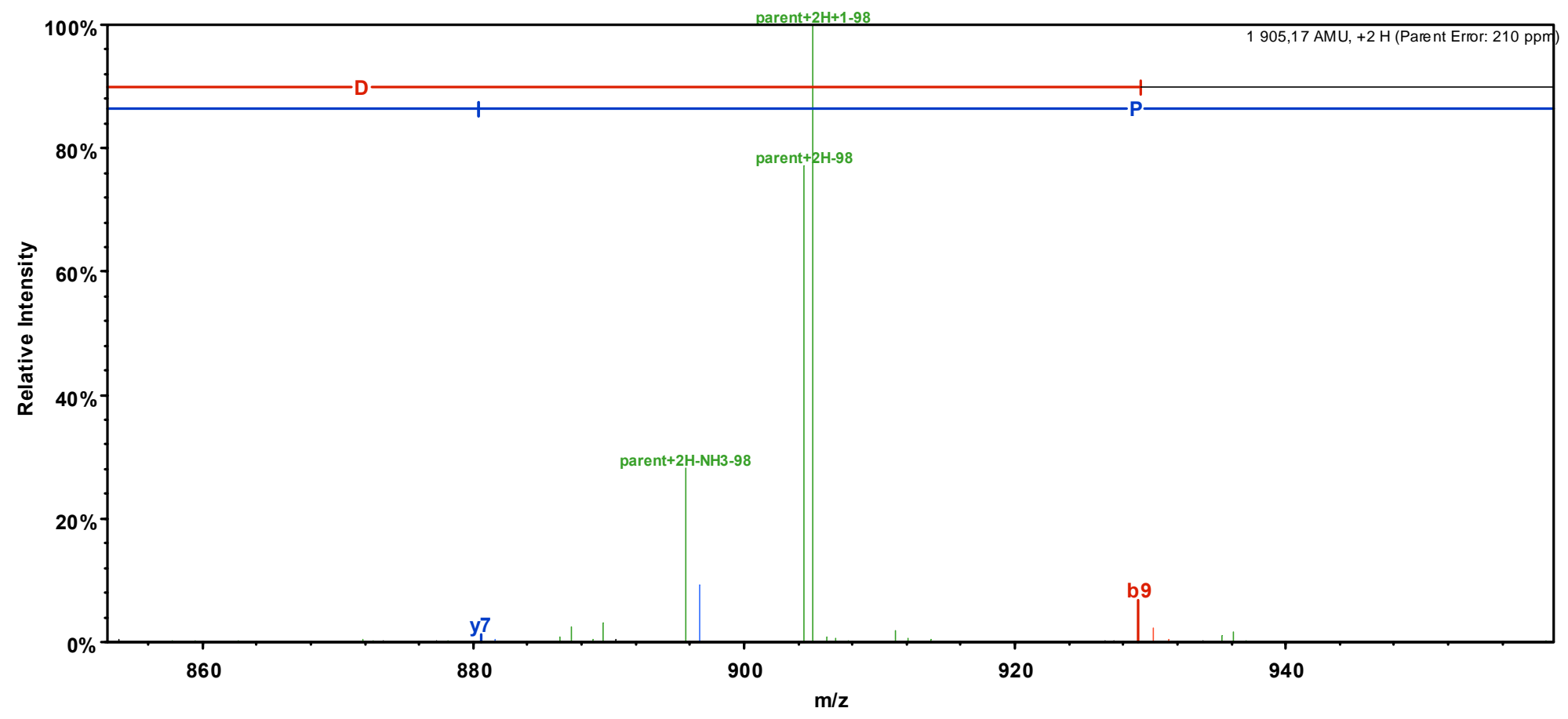




\section{$\underline{\text { Left of precursor }}$}

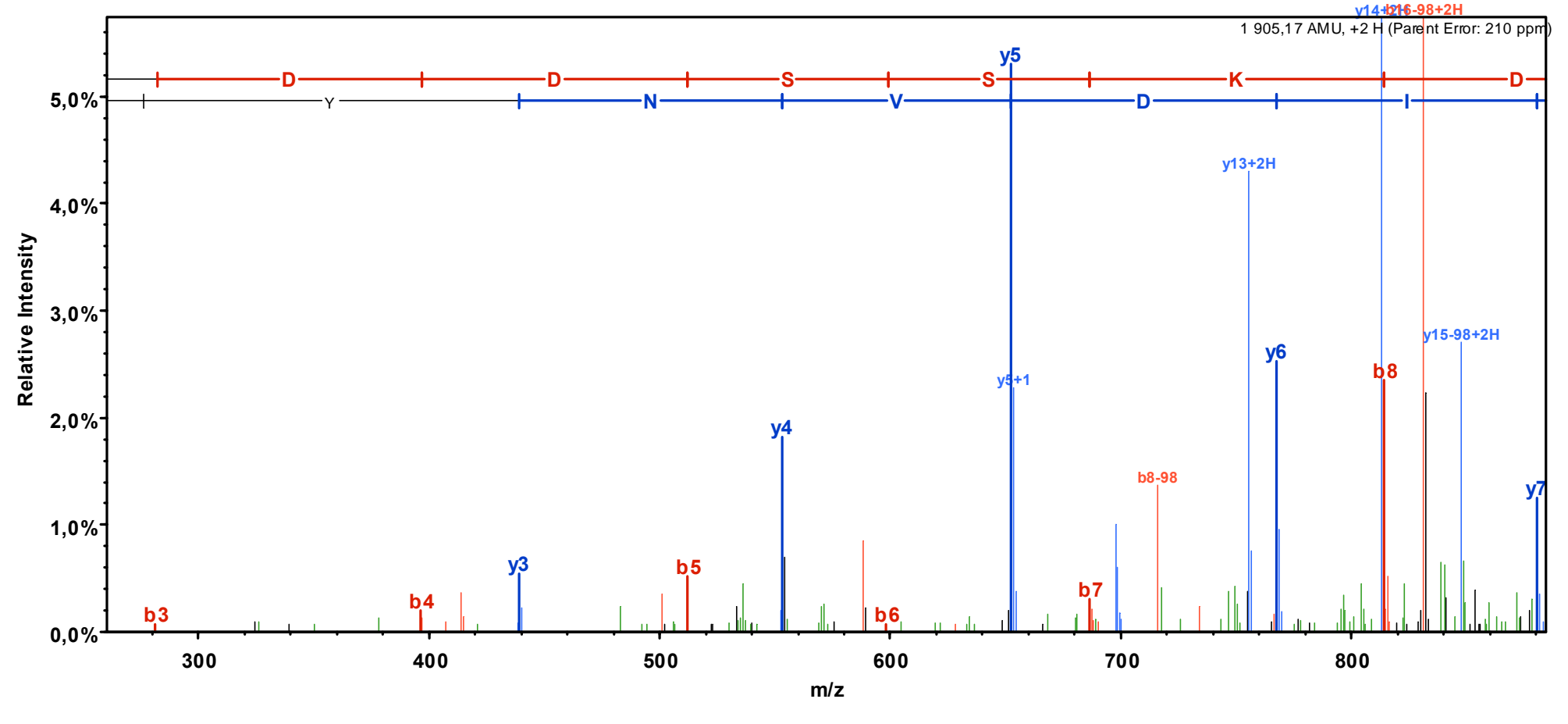

\section{$\underline{\text { Right of precursor }}$}

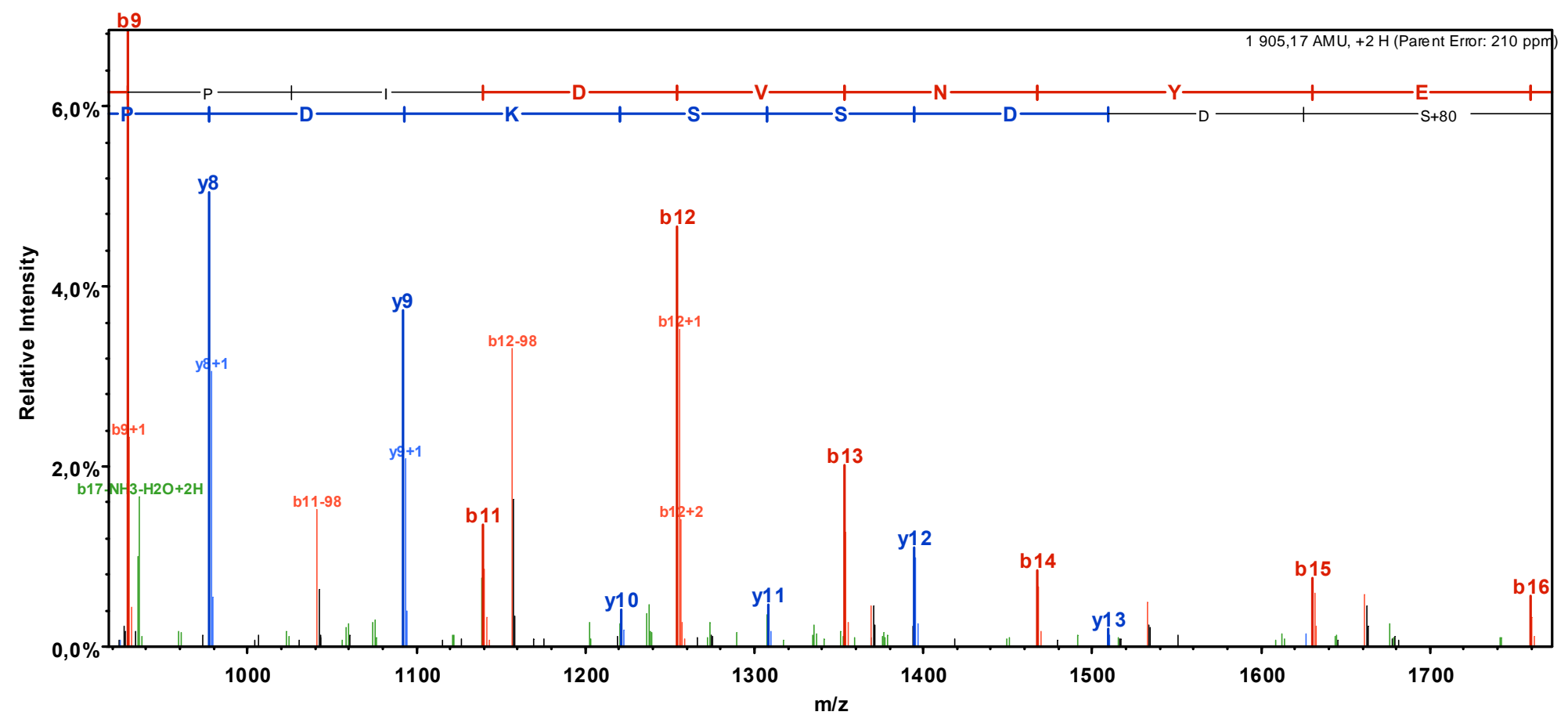




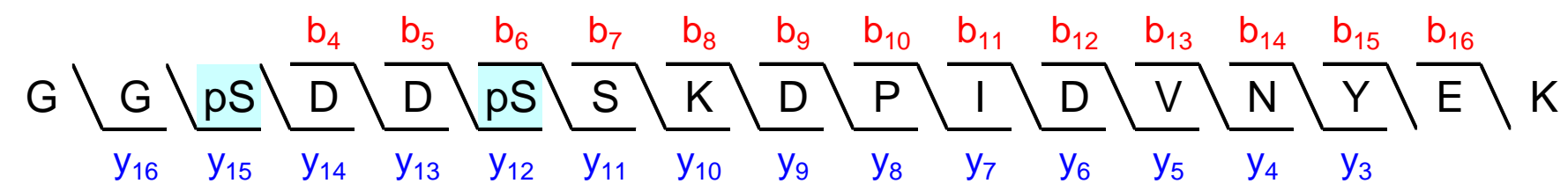

Sequest: $X$ Corr $=5.46, \mathrm{dCn} 1=0.11$ (between $1^{\text {st }}$ and $2^{\text {nd }}$ best matches), $\mathrm{dCn} 2=0.26$ (between $2^{\text {nd }}$ and $3^{\text {rd }}$ best matches). $A_{\text {score }}=48.32$ (first phosphorylation), 11.51 (second phosphorylation).

Sequest's second best match with XCorr $=4.85$ is to the same peptide sequence but with an alternative phosphorylation site: GGPSDDSPSKDPIDVNYEK.

The weak Ascore for the second phosphorylation indicates that, given the observed MS/MS spectrum, the phosphopeptide identified could either be Sequest's first or second best match (Nat Biotechnol. 2006;24(10):1285-92).

\section{MS/MS spectrum's fragmentation table}

\begin{tabular}{|c|c|c|c|c|c|c|c|c|c|c|}
\hline B & B Ions & $\mathrm{B}+2 \mathrm{H}$ & $\mathrm{B}-\mathrm{NH} 3$ & $\mathrm{~B}-\mathrm{H} 2 \mathrm{O}$ & AA & $Y$ Ions & $\mathrm{Y}+2 \mathrm{H}$ & $\mathrm{Y}-\mathrm{NH} 3$ & $\mathrm{Y}-\mathrm{H} 2 \mathrm{O}$ & $Y$ \\
\hline 1 & 58,0 & & & & G & 1985,7 & 993.4 & 1968.7 & 1967.7 & 17 \\
\hline 2 & 115,1 & & & & G & 1928.7 & 964.9 & 1911,7 & 1910,7 & 16 \\
\hline 3 & 282,0 & & & 264,0 & $S+80$ & 1871,7 & 936.4 & 1854,7 & 1853,7 & 15 \\
\hline 4 & 397.1 & & & 379,1 & D & 1704,7 & 852.9 & 1687,7 & 1686.7 & 14 \\
\hline 5 & 512.1 & & & 494,1 & D & 1589.7 & 795.3 & 1572,6 & 1571,7 & 13 \\
\hline 6 & 679.1 & 340,1 & & 661.1 & $\mathbf{S + 8 0}$ & 1474.6 & 737,8 & 1457.6 & 1456.6 & 12 \\
\hline 7 & 766.1 & 383.6 & & 748.1 & S & 1307.6 & 654,3 & 1290.6 & 1289,6 & 11 \\
\hline 8 & 894.2 & 447,6 & 877.2 & 876.2 & K & 1220.6 & 610,8 & 1203.6 & 1202,6 & 10 \\
\hline 9 & 1009.3 & 505.1 & 992,2 & 991,2 & D & 1092.5 & 546,8 & 1075.5 & 1074,5 & 9 \\
\hline 10 & 1106.3 & 553.7 & 1089.3 & 1088,3 & $\mathbf{P}$ & 977.5 & 489,3 & 960.5 & 959.5 & 8 \\
\hline 11 & 1219.4 & 610.2 & 1202,4 & 1201.4 & I & 880.4 & 440,7 & 863.4 & 862,4 & 7 \\
\hline 12 & 1334.4 & 667.7 & 1317.4 & 1316.4 & D & 767,4 & 384,2 & 750.3 & 749,3 & 6 \\
\hline 13 & 1433.5 & 717.2 & 1416.5 & 1415.5 & V & 652.3 & & 635,3 & 634,3 & 5 \\
\hline 14 & 1547.5 & 774.3 & 1530.5 & 1529.5 & $\mathrm{~N}$ & 553.3 & & 536,2 & 535,3 & 4 \\
\hline 15 & 1710.6 & 855.8 & 1693.6 & 1692,6 & $\mathbf{Y}$ & 439.2 & & 422,2 & 421.2 & 3 \\
\hline 16 & 1839.6 & 920.3 & 1822,6 & 1821,6 & $\mathrm{E}$ & 276,2 & & 259,1 & 258,1 & 2 \\
\hline 17 & 1985,7 & 993,4 & 1968,7 & 1967.7 & K & 147,1 & & 130,1 & & 1 \\
\hline
\end{tabular}




\section{MS/MS spectrum}

\section{Full-range}

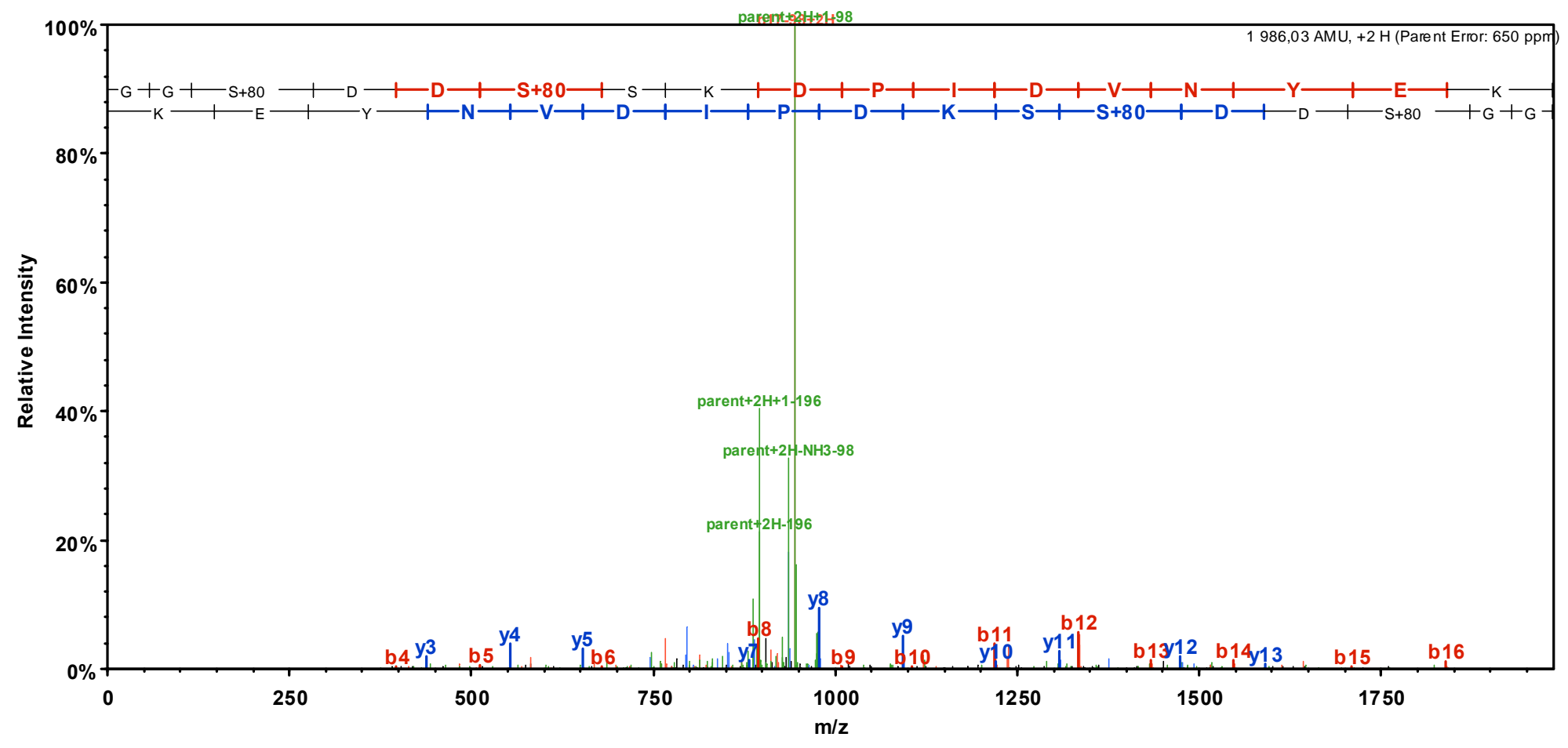

\section{Close to precursor}

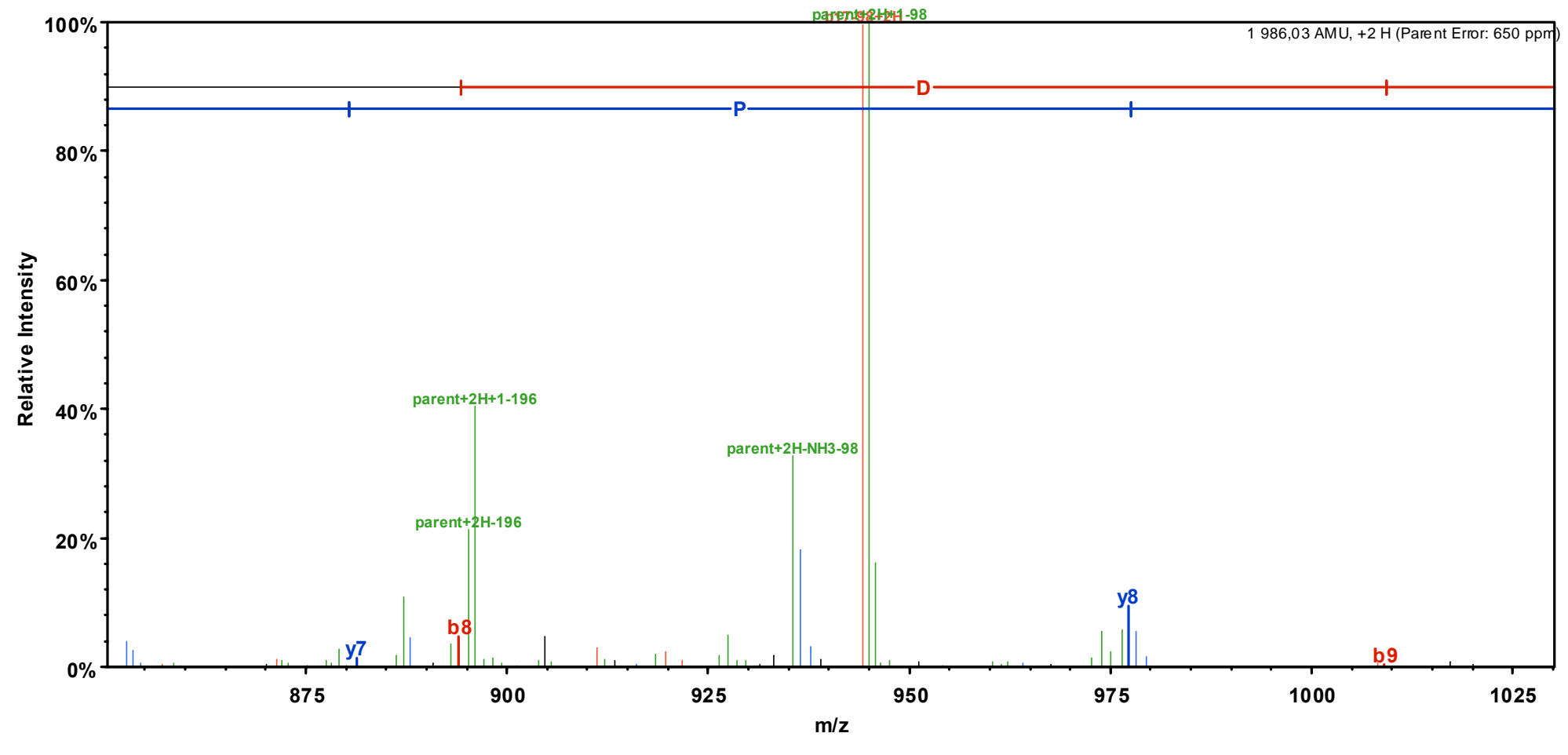




\section{Left of precursor}

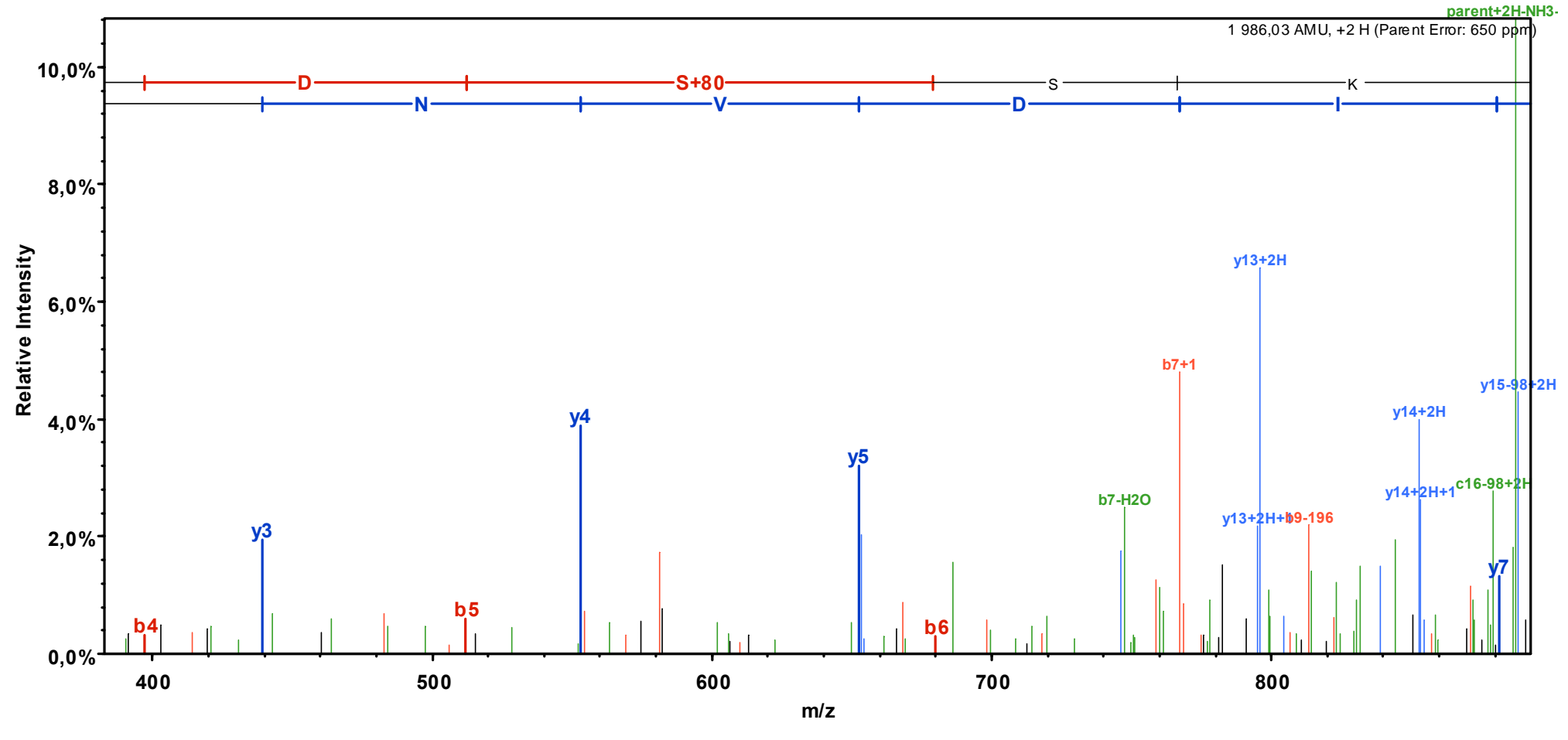

\section{$\underline{\text { Right of precursor }}$}

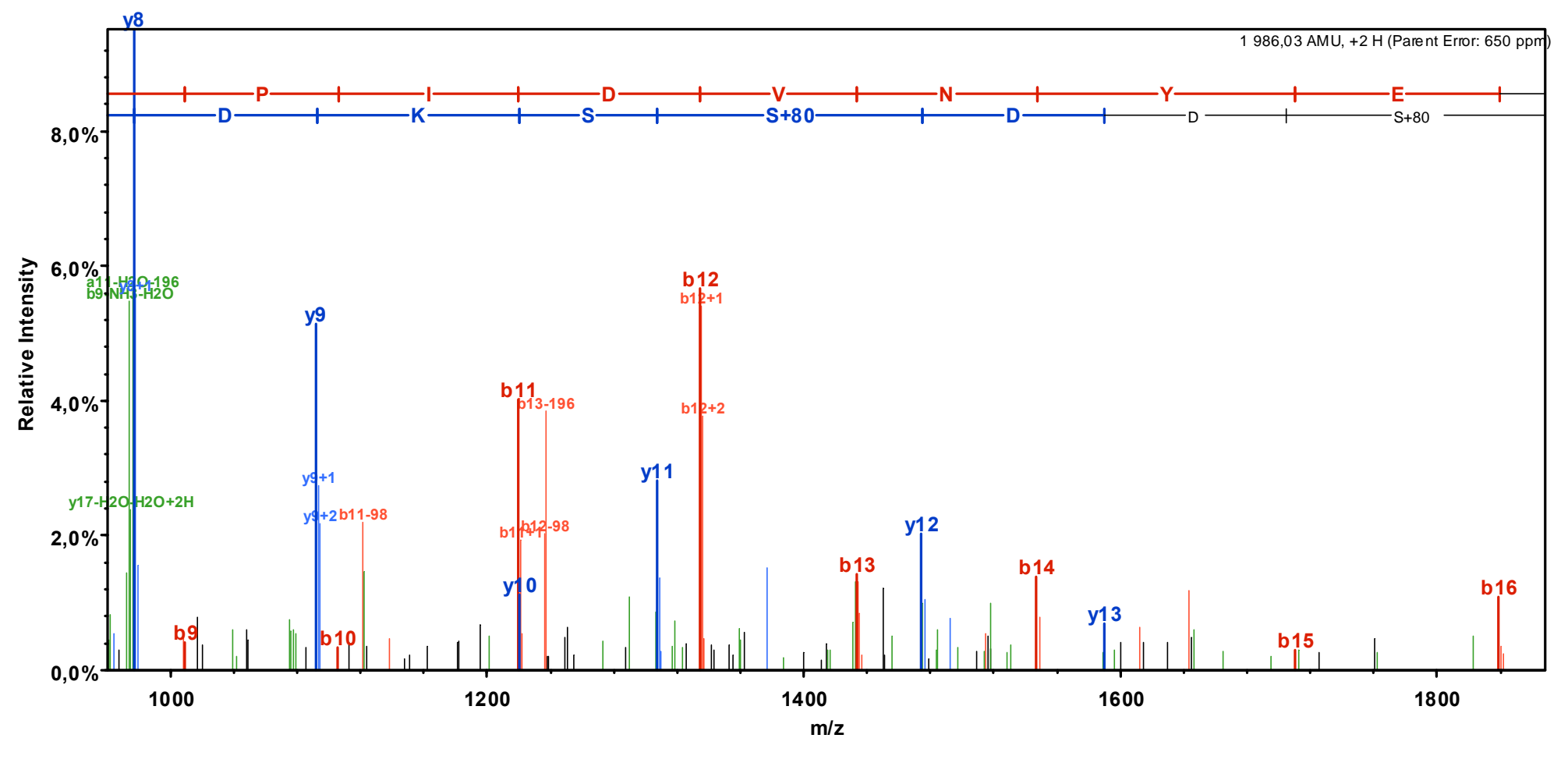


Supplementary mass spectrometric data:

PARP-1, Protein Kinase C $\beta$-phosphorylated

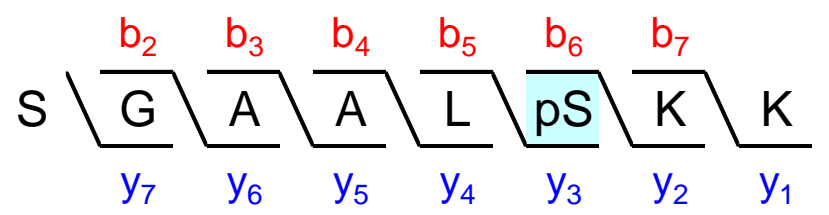

Sequest: XCorr $=1.51, \mathrm{dCn}=0.67$.

Ascore $=75.46$

Phosphorylation site is localized with $\geq 99 \%$ certainty as Ascore $\geq 20$ (Nat Biotechnol. 2006;24(10):1285-92).

MS/MS spectrum's fragmentation table

\begin{tabular}{|c|c|c|c|c|c|c|c|c|c|c|}
\hline B & $B$ Ions & $\mathrm{B}+2 \mathrm{H}$ & $\mathrm{B}-\mathrm{NH} 3$ & $\mathrm{~B}-\mathrm{H} 2 \mathrm{O}$ & AA & $Y$ Ions & $\mathrm{Y}+2 \mathrm{H}$ & $\mathrm{Y}-\mathrm{NH} 3$ & $\mathrm{Y}-\mathrm{H} 2 \mathrm{O}$ & Y \\
\hline 1 & 88,0 & & & 70,0 & $S$ & 841,4 & 421,2 & 824,4 & 823,4 & 8 \\
\hline 2 & 145.1 & & & 127,1 & G & 754,4 & 377.7 & 737.4 & 736.4 & 7 \\
\hline 3 & 216.1 & & & 198.1 & A & 697.4 & 349.2 & 680.3 & 679.4 & 6 \\
\hline 4 & 287.1 & & & 269,1 & A & 626.3 & 313.7 & 609,3 & 608.3 & 5 \\
\hline 5 & 400.2 & & & 382,2 & L & 555.3 & 278.1 & 538,3 & 537.3 & 4 \\
\hline 6 & 567.2 & 284,1 & & 549,2 & $S+80$ & 442.2 & 221,6 & 425,2 & 424,2 & 3 \\
\hline 7 & 695.3 & 348.2 & 678.3 & 677,3 & K & 275.2 & 138.1 & 258.2 & & 2 \\
\hline 8 & 841,4 & 421,2 & 824,4 & 823,4 & K & 147.1 & & 130.1 & & 1 \\
\hline
\end{tabular}




\section{MS/MS spectrum}

Full-range

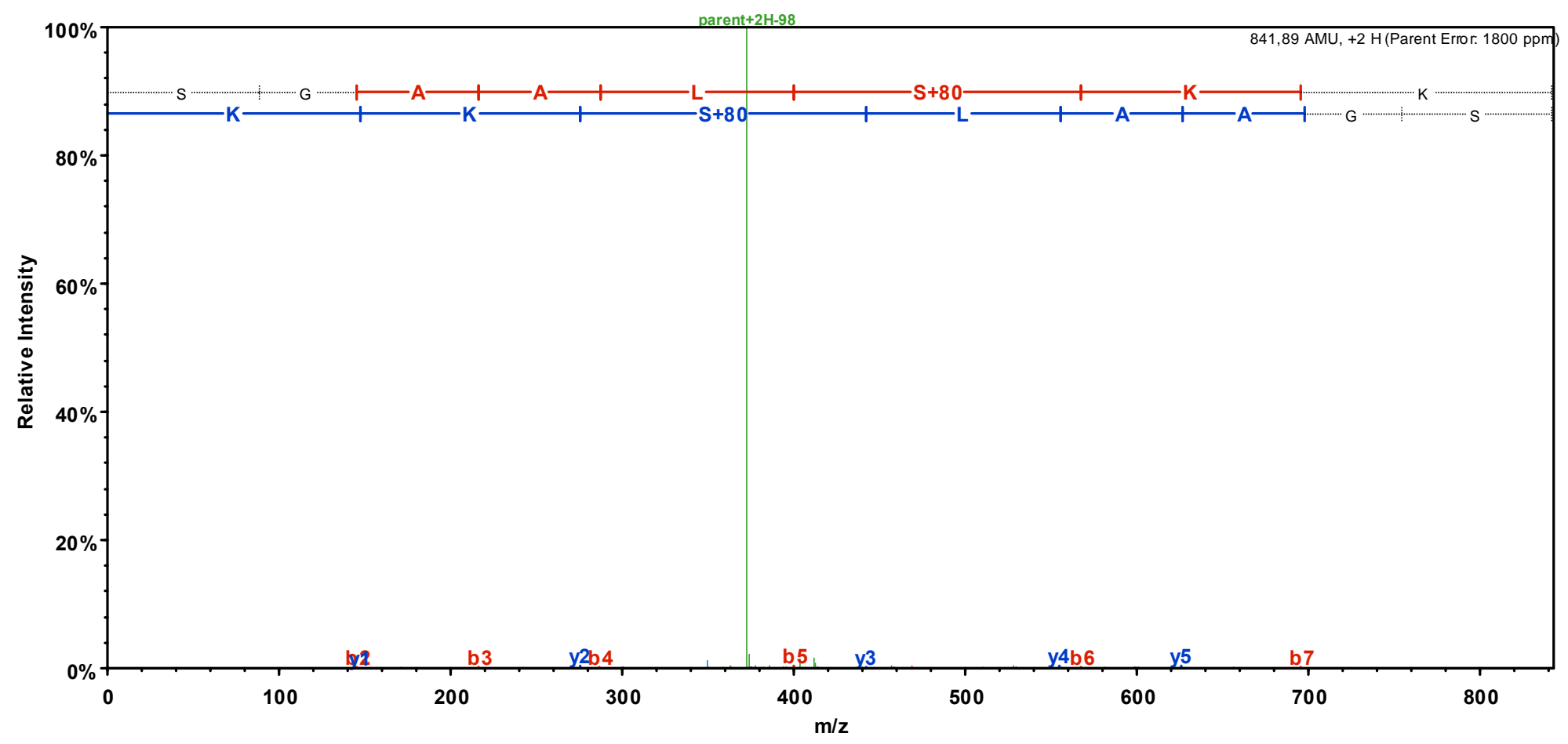

\section{$\underline{\text { Close to precursor }}$}

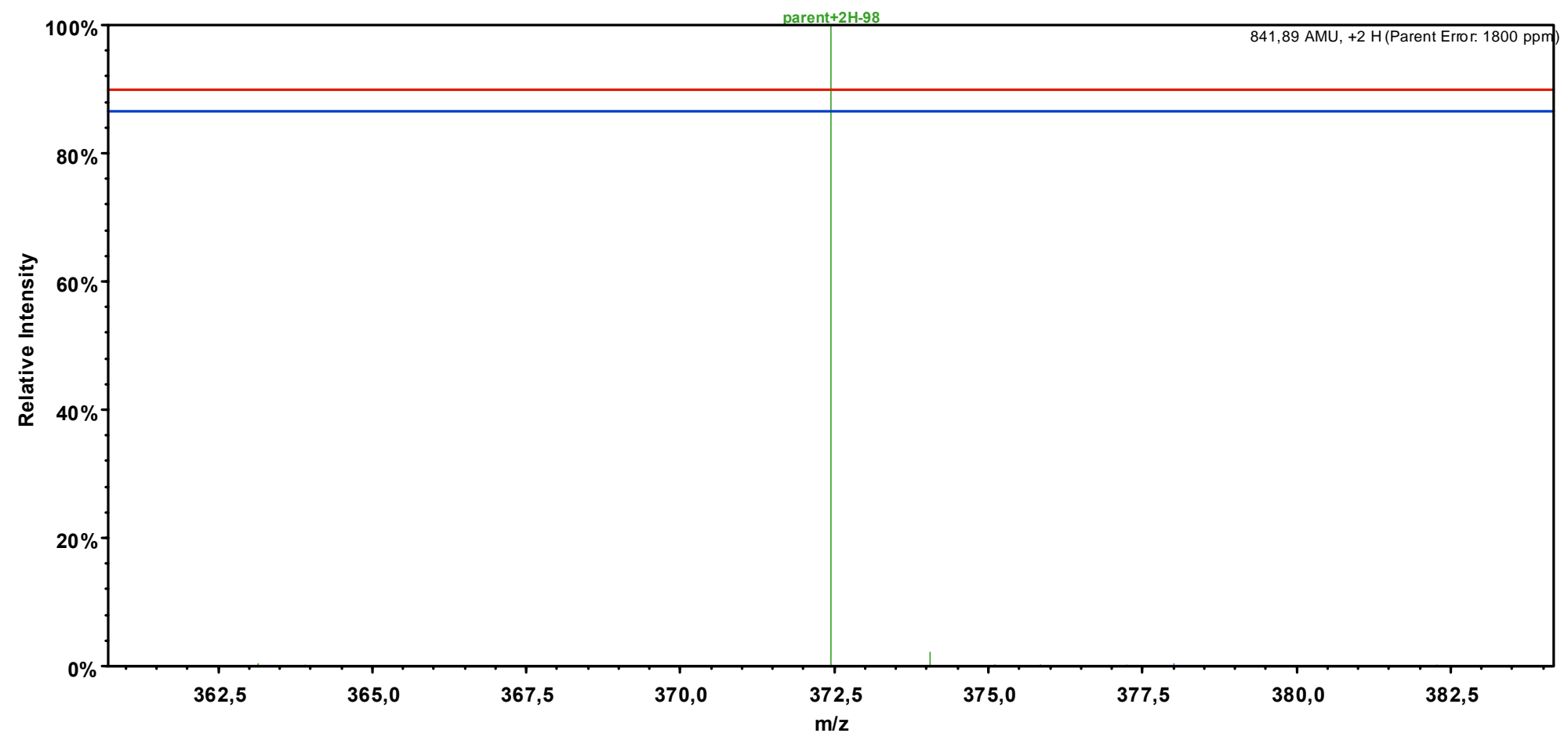




\section{Left of precursor}

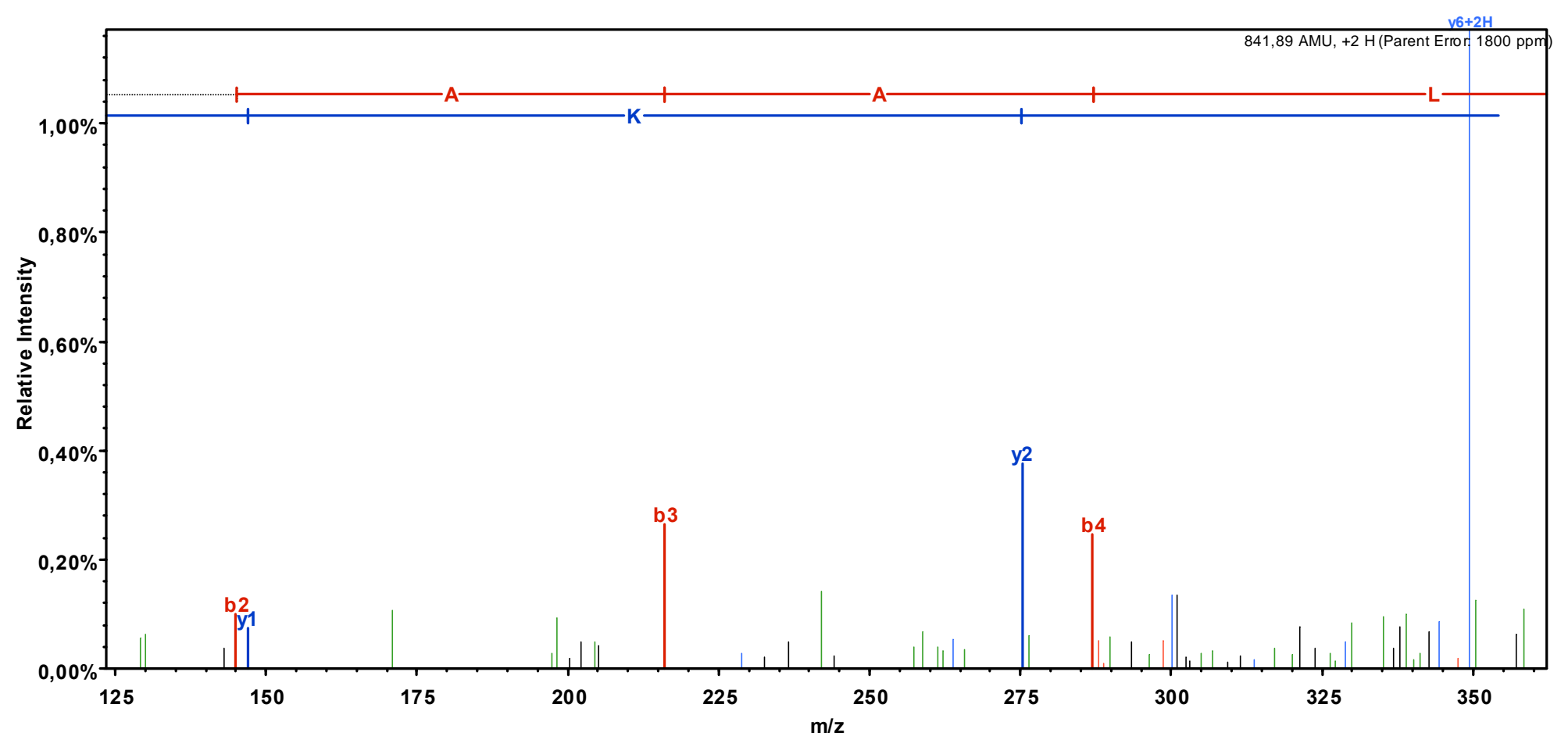

\section{$\underline{\text { Right of precursor }}$}

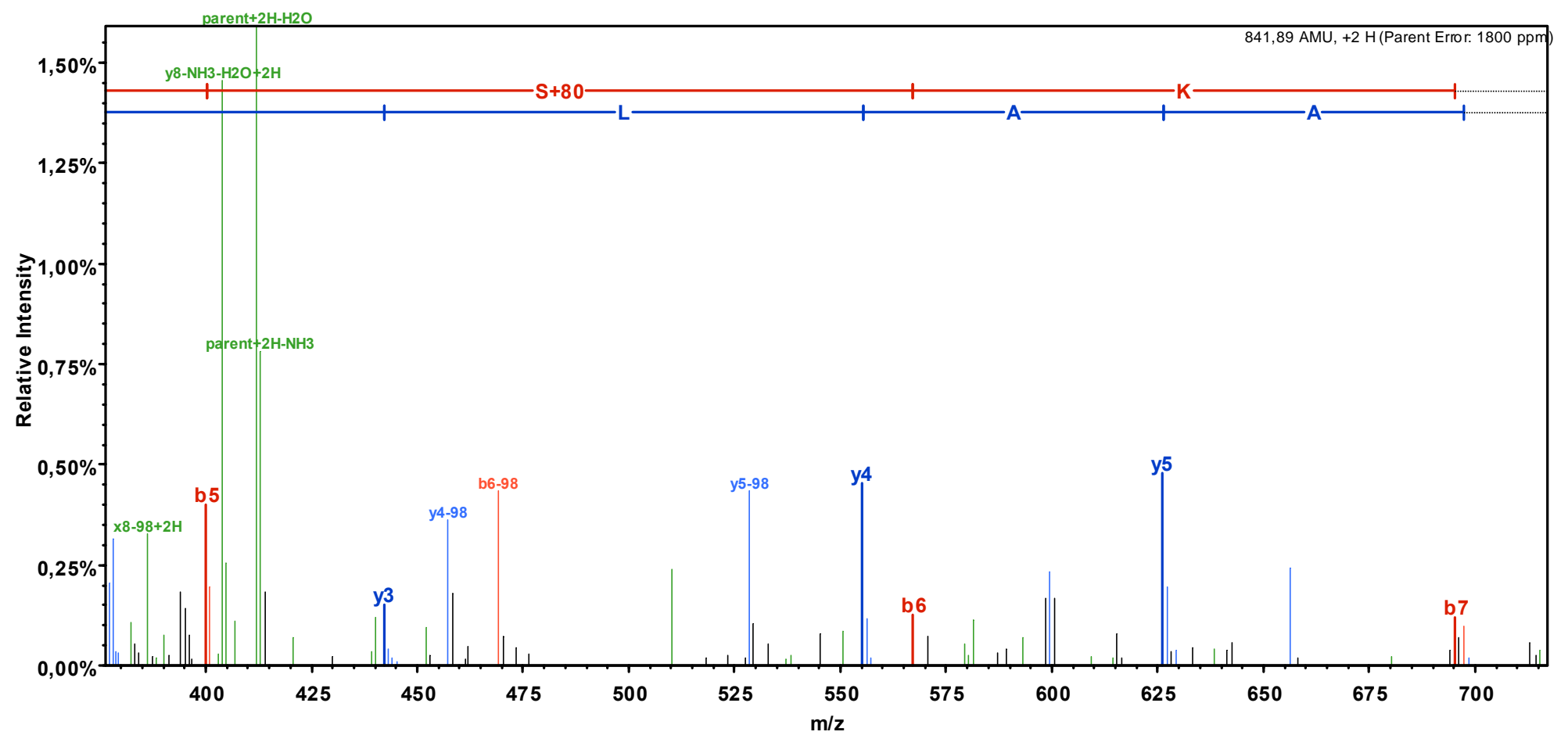




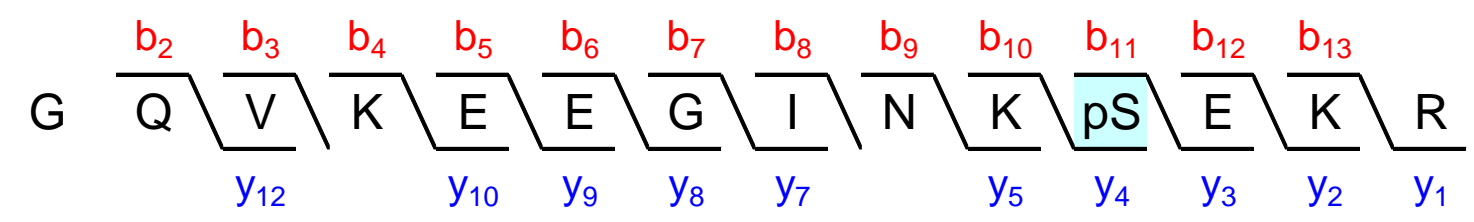

Sequest: XCorr $=3.92, \mathrm{dCn}=0.43$.

Ascore $=$ Not available (only one possible phosphorylation site).

The phosphorylation is located at the only possible phosphorylation site (no other S, T, Y within the peptide).

\section{MS/MS spectrum's fragmentation table}

\begin{tabular}{|c|c|c|c|c|c|c|c|c|c|c|}
\hline B & B Ions & $\mathrm{B}+2 \mathrm{H}$ & $\mathrm{B}-\mathrm{NH} 3$ & $\mathrm{~B}-\mathrm{H} 2 \mathrm{O}$ & AA & $Y$ Ions & $\mathrm{Y}+2 \mathrm{H}$ & $\mathrm{Y}-\mathrm{NH} 3$ & $\mathrm{Y}-\mathrm{H} 2 \mathrm{O}$ & $Y$ \\
\hline 1 & 58,0 & & & & G & 1681,8 & 841,4 & 1664,8 & 1663,8 & 14 \\
\hline 2 & 186.1 & & 169.1 & & $\mathbf{Q}$ & 1624,8 & 812,9 & 1607,8 & 1606,8 & 13 \\
\hline 3 & 285.2 & & 268.1 & & V & 1496,7 & 748.9 & 1479,7 & 1478,7 & 12 \\
\hline 4 & 413.3 & 207,1 & 396,2 & & K & 1397,7 & 699,3 & 1380,7 & 1379,7 & 11 \\
\hline 5 & 542.3 & 271.7 & 525.3 & 524,3 & E & 1269,6 & 635.3 & 1252,6 & 1251,6 & 10 \\
\hline 6 & 671.3 & 336.2 & 654.3 & 653.3 & E & 1140,5 & 570.8 & 1123,5 & 1122,5 & 9 \\
\hline 7 & 728.4 & 364.7 & 711,3 & 710.3 & G & 1011,5 & 506.3 & 994,5 & 993,5 & 8 \\
\hline 8 & 841.4 & 421.2 & 824.4 & 823.4 & I & 954,5 & 477.7 & 937,5 & 936,5 & 7 \\
\hline 9 & 955.5 & 478.2 & 938.5 & 937,5 & $\mathrm{~N}$ & 841,4 & 421,2 & 824,4 & 823,4 & 6 \\
\hline 10 & 1083.6 & 542,3 & 1066.6 & 1065,6 & K & 727.4 & 364.2 & 710,3 & 709,3 & 5 \\
\hline 11 & 1250,6 & 625.8 & 1233,6 & 1232,6 & $S+80$ & 599.3 & 300.1 & 582,2 & 581.2 & 4 \\
\hline 12 & 1379,6 & 690.3 & 1362,6 & 1361,6 & E & 432.3 & 216,6 & 415.2 & 414,2 & 3 \\
\hline 13 & 1507,7 & 754.4 & 1490,7 & 1489,7 & K & 303.2 & 152,1 & 286.2 & & 2 \\
\hline 14 & 1681,8 & 841,4 & 1664,8 & 1663,8 & $\mathbf{R}$ & 175.1 & & 158,1 & & 1 \\
\hline
\end{tabular}




\section{MS/MS spectrum}

\section{Full-range}

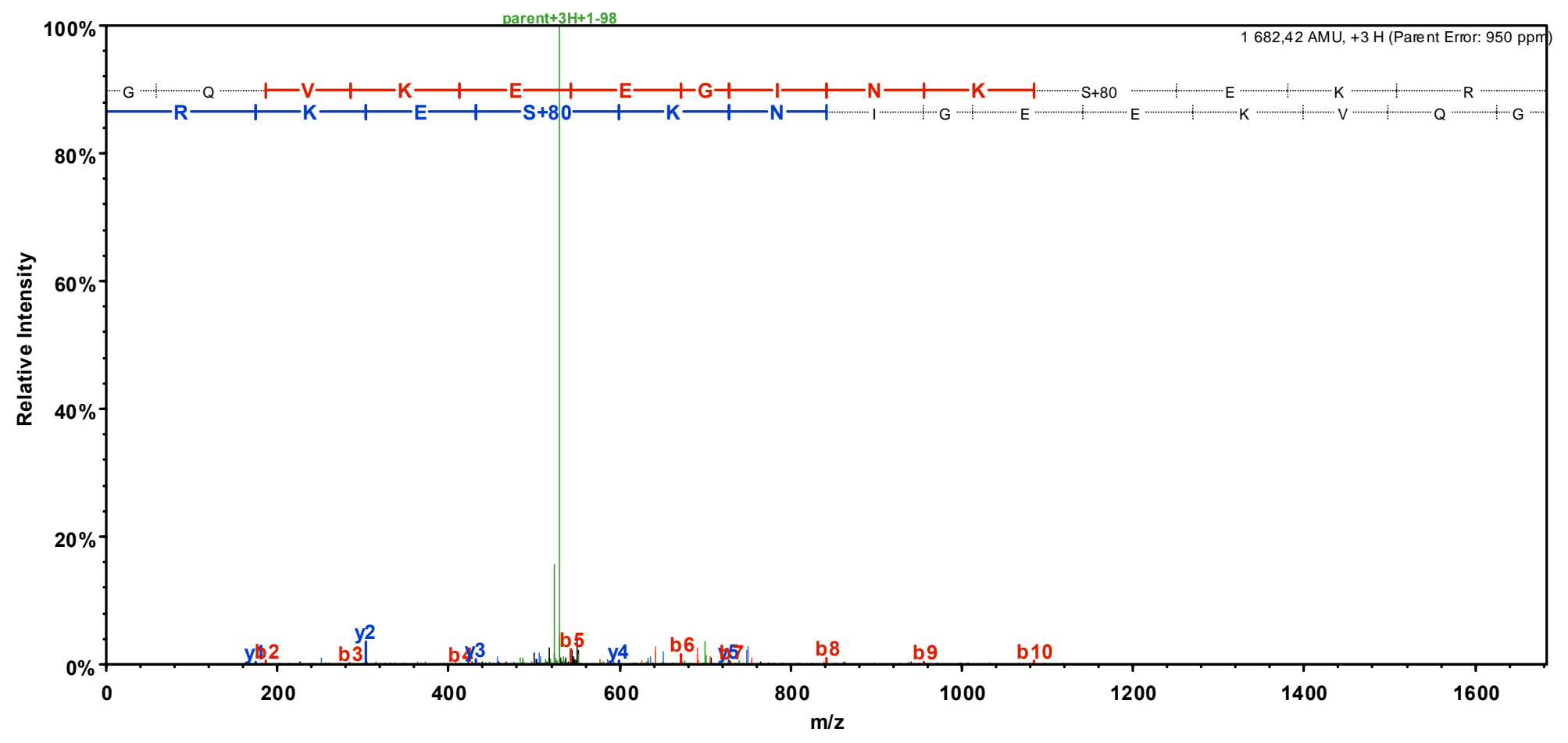

\section{Close to precursor}

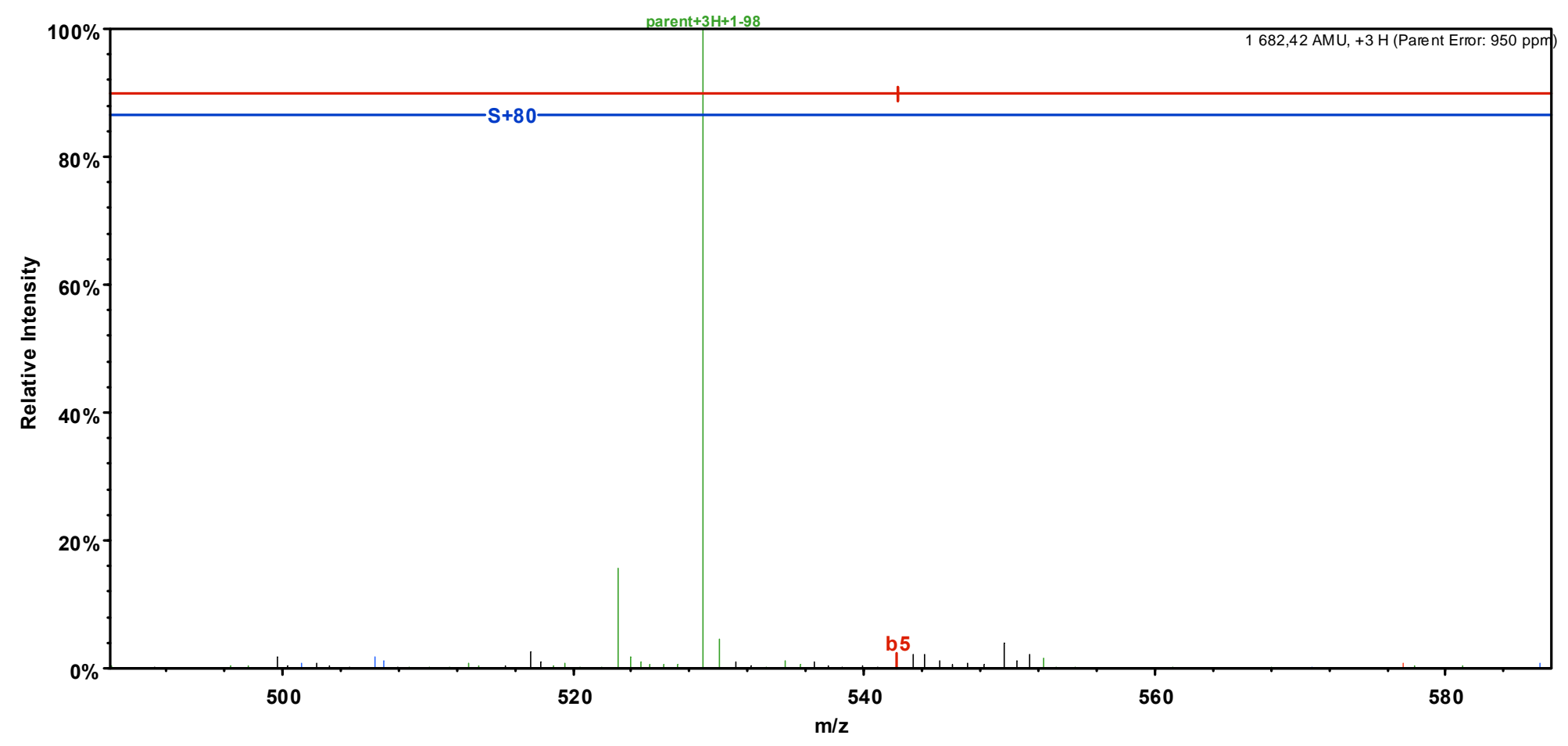




\section{Left of precursor}

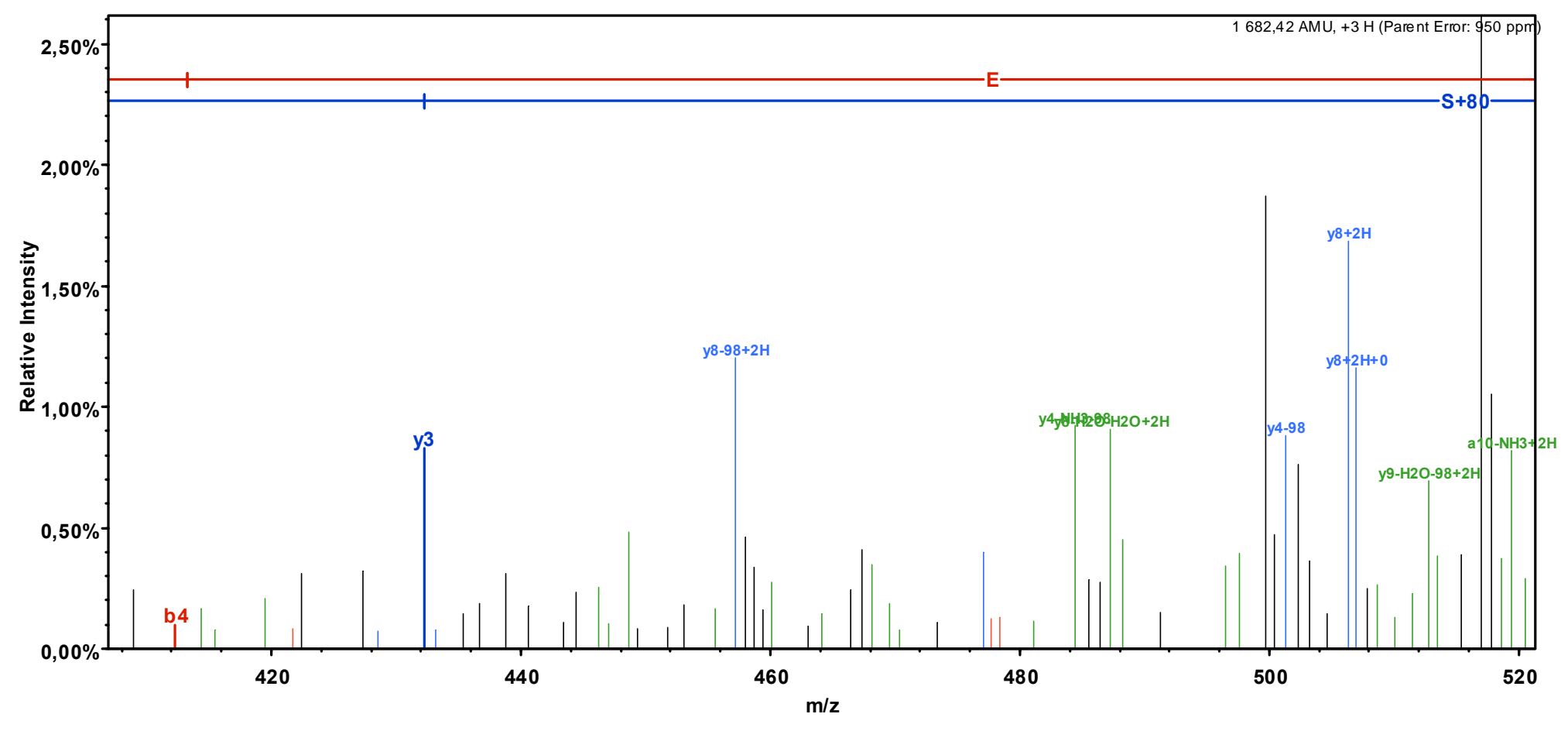

\section{$\underline{\text { Right of precursor }}$}

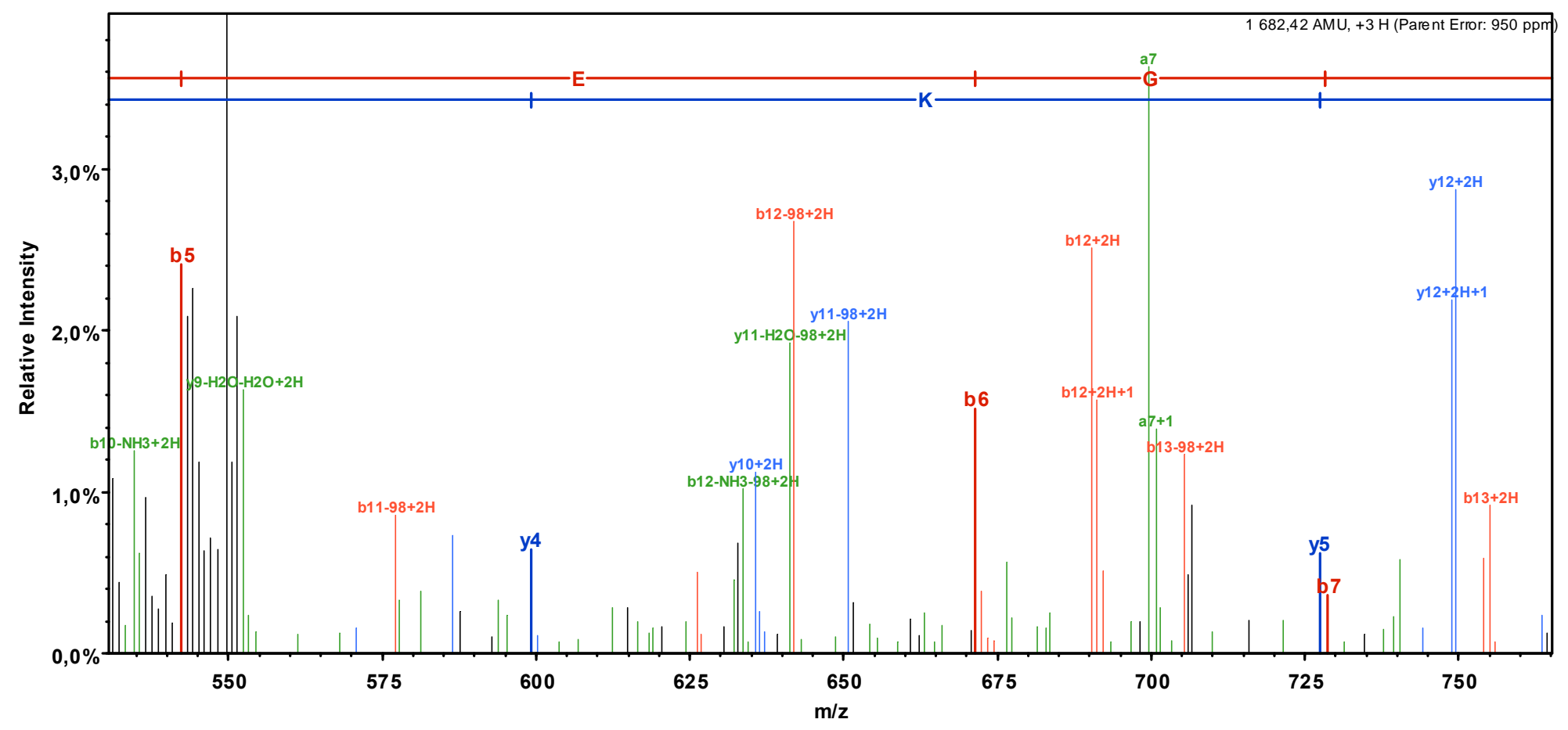




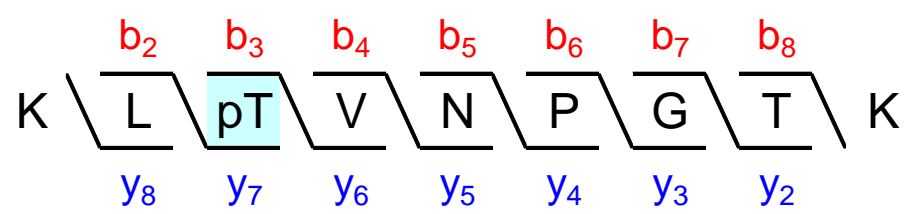

Sequest: XCorr $=2.45, \mathrm{dCn}=0.74$.

Ascore $=21.23$.

Phosphorylation site is localized with $\geq 99 \%$ certainty as Ascore $\geq 20$ (Nat Biotechnol. 2006;24(10):1285-92).

\section{MS/MS spectrum's fragmentation table}

\begin{tabular}{|c|c|c|c|c|c|c|c|c|c|c|}
\hline B & B Ions & $\mathrm{B}+2 \mathrm{H}$ & $\mathrm{B}-\mathrm{NH} 3$ & $\mathrm{~B}-\mathrm{H} 2 \mathrm{O}$ & AA & $Y$ Ions & $\mathrm{Y}+2 \mathrm{H}$ & $\mathrm{Y}-\mathrm{NH} 3$ & $\mathrm{Y}-\mathrm{H} 2 \mathrm{O}$ & $Y$ \\
\hline 1 & 129,1 & 65.1 & 112,1 & & $K$ & 1037,5 & 519,3 & 1020.5 & 1019,5 & 9 \\
\hline 2 & 242.2 & 121,6 & 225.2 & & $\mathbf{L}$ & 909.4 & 455.2 & 892,4 & 891,4 & 8 \\
\hline 3 & 423.2 & 212,1 & 406.2 & 405,2 & $\mathrm{~T}+\mathbf{8 0}$ & 796.4 & 398.7 & 779,3 & 778.4 & 7 \\
\hline 4 & 522,3 & 261.6 & 505,2 & 504.3 & V & 615.3 & 308.2 & 598,3 & 597.3 & 6 \\
\hline 5 & 636.3 & 318.7 & 619.3 & 618,3 & $\mathbf{N}$ & 516,3 & & 499.3 & 498,3 & 5 \\
\hline 6 & 733.4 & 367.2 & 716.3 & 715,4 & $\mathbf{P}$ & 402.2 & & 385.2 & 384,2 & 4 \\
\hline 7 & 790.4 & 395,7 & 773.4 & 772,4 & G & 305.2 & & 288.2 & 287.2 & 3 \\
\hline 8 & 891.4 & 446.2 & 874,4 & 873.4 & $\mathbf{T}$ & 248.2 & & 231.1 & 230.2 & 2 \\
\hline 9 & 1037.5 & 519,3 & 1020.5 & 1019,5 & K & 147,1 & & 130,1 & & 1 \\
\hline
\end{tabular}




\section{MS/MS spectrum}

\section{Full-range}

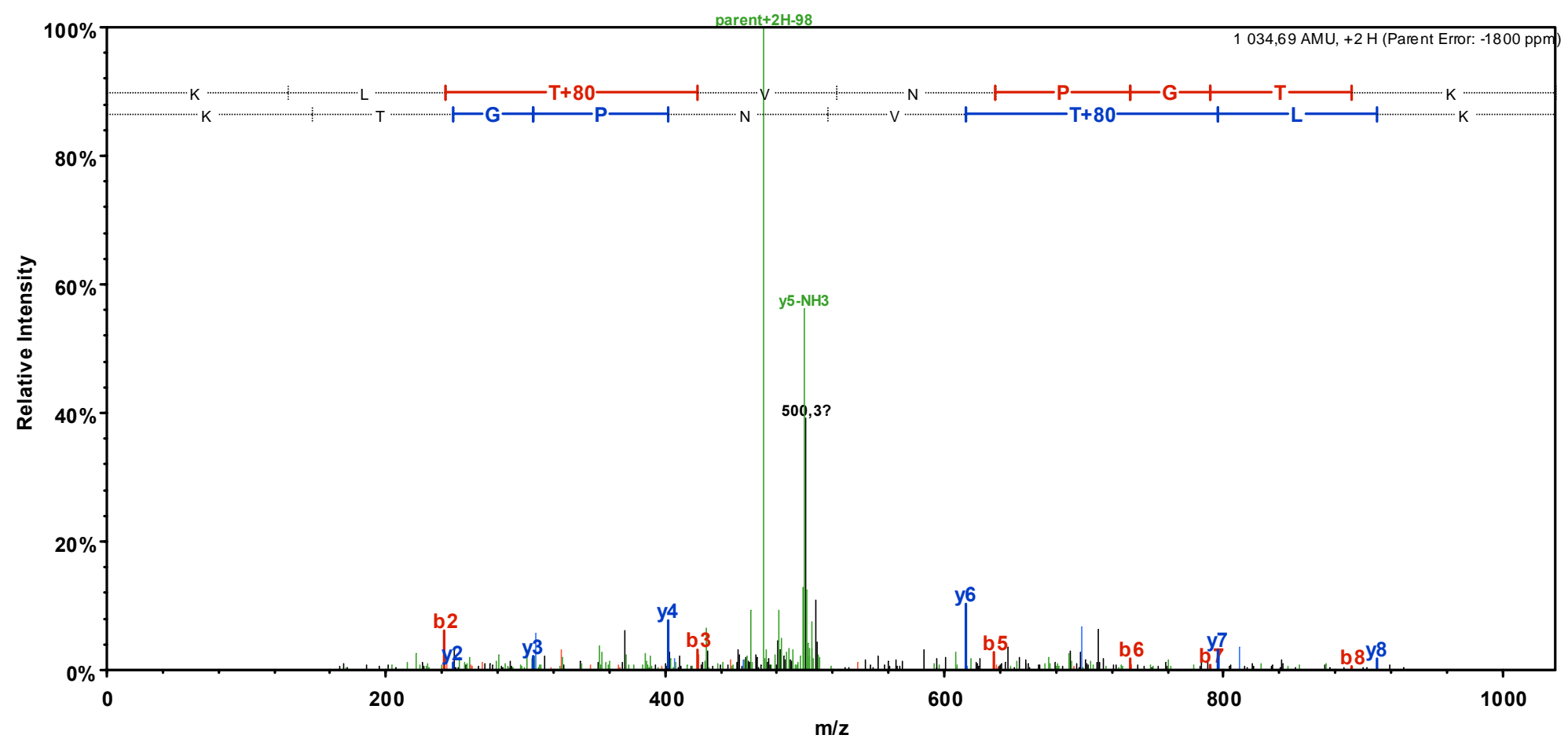

\section{$\underline{\text { Close to precursor }}$}

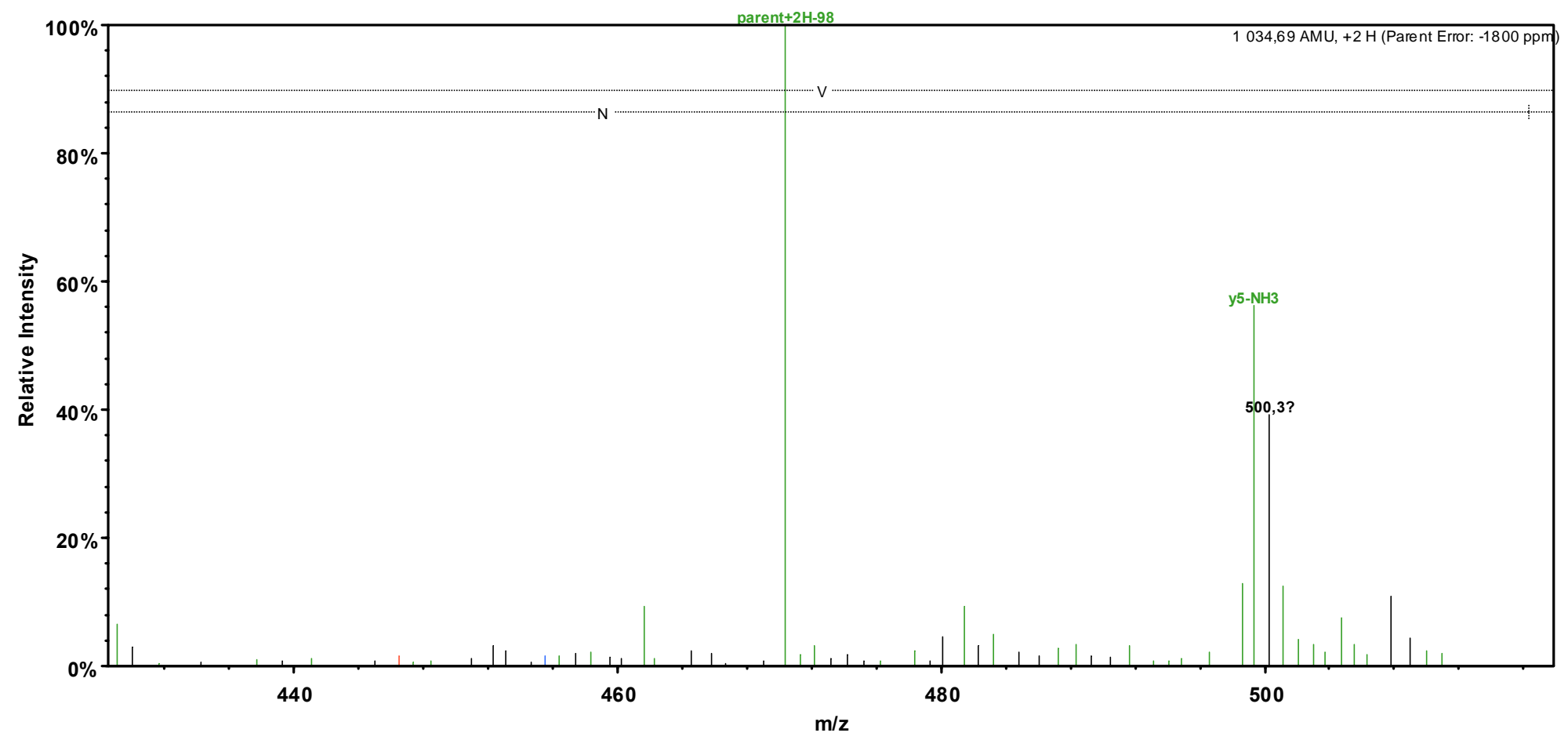




\section{Left of precursor}

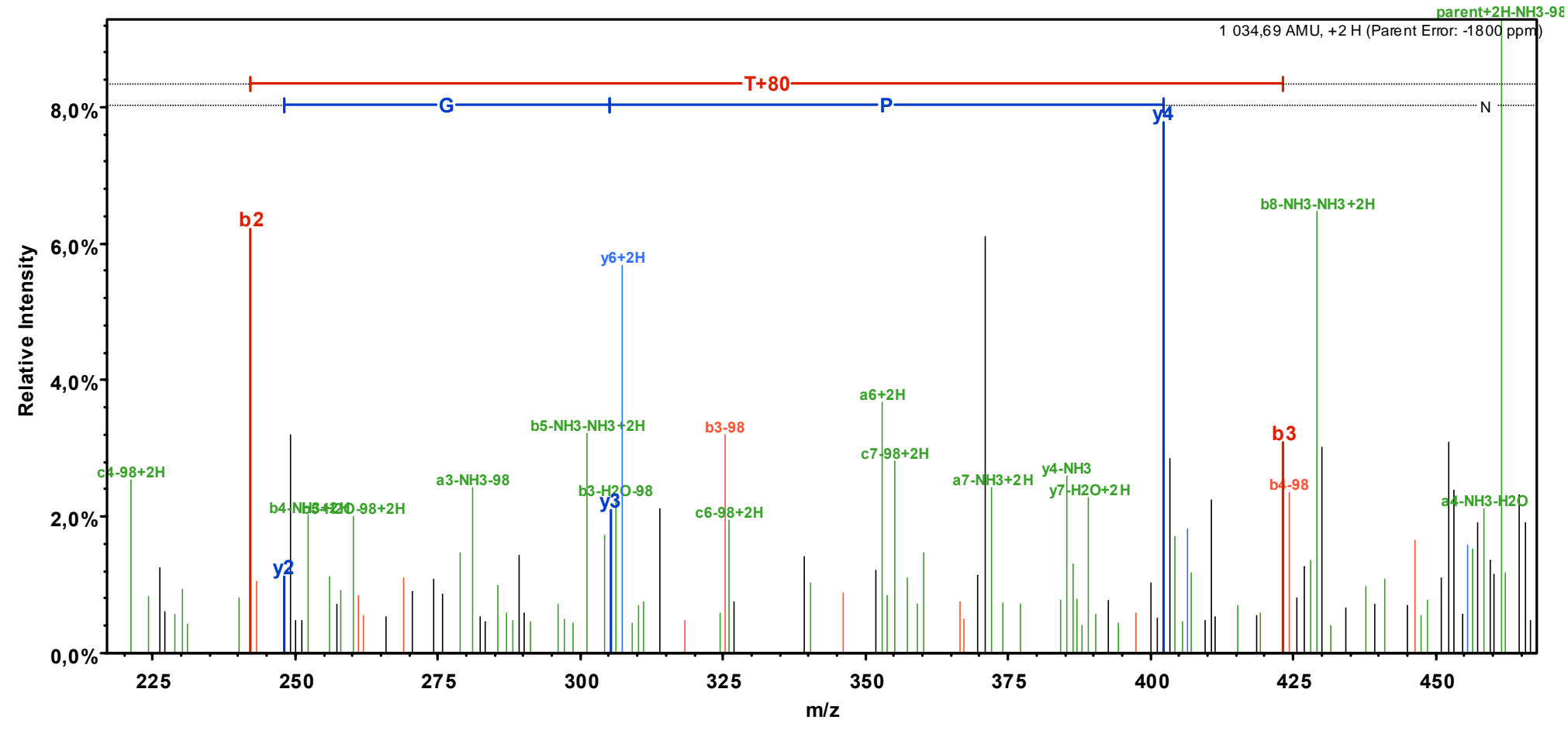

\section{$\underline{\text { Right of precursor }}$}

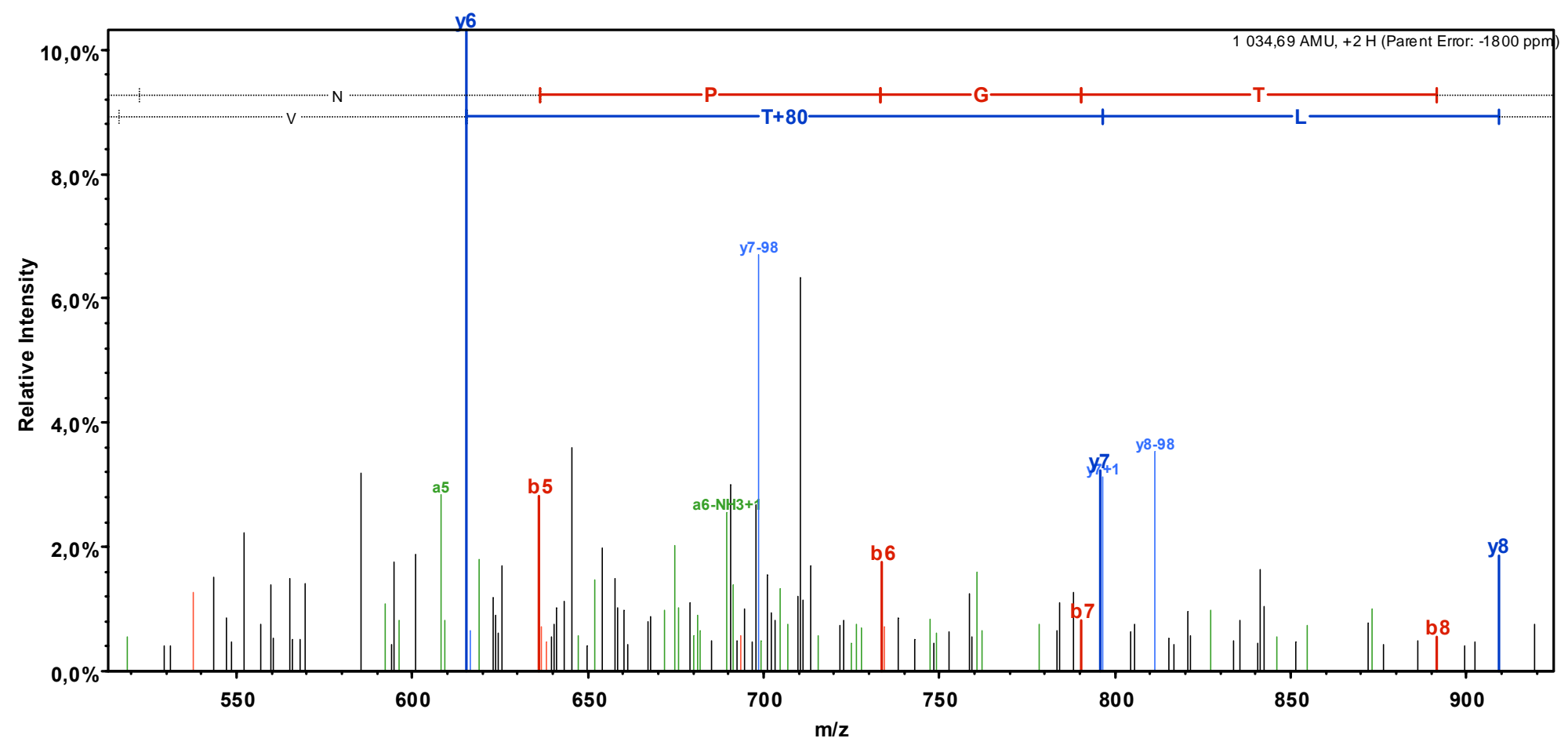




\section{Supplementary mass spectrometric data:}

PARP-1,

\section{phosphorylated with Ca2+/calmodulin-dependent protein kinase-II (CaMK II)}

\section{Spectrum 8170.2475.2475.2.dta (charge state: +2 )}

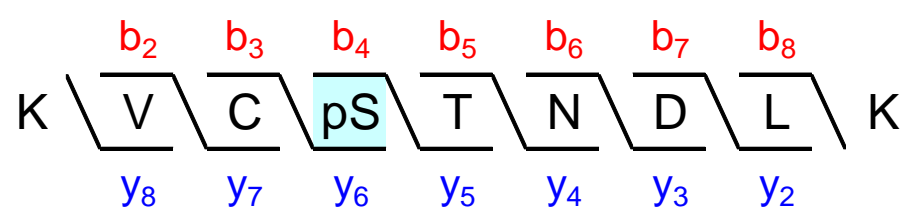

Sequest: $X$ Corr $=2.24, \mathrm{dCn} 1=0.08$ (between $1^{\text {st }}$ and $2^{\text {nd }}$ best matches), $\mathrm{dCn} 2=0.64$ (between $2^{\text {nd }}$ and $3^{\text {rd }}$ best matches). $A_{\text {score }}=0$.

Sequest's second best match with XCorr $=2.05$ is to the same peptide sequence but with an alternative phosphorylation site: KVCSPTNDLK.

The very weak Ascore indicates that, given the observed MS/MS spectrum, the phosphopeptide identified could either be Sequest's first or second best match (Nat Biotechnol. 2006;24(10):1285-92).

\section{MS/MS spectrum's fragmentation table}

\begin{tabular}{|c|c|c|c|c|c|c|c|c|c|c|}
\hline B & B Ions & $\mathrm{B}+2 \mathrm{H}$ & $\mathrm{B}-\mathrm{NH} 3$ & $\mathrm{~B}-\mathrm{H} 2 \mathrm{O}$ & AA & $Y$ Ions & $\mathrm{Y}+2 \mathrm{H}$ & $\mathrm{Y}-\mathrm{NH} 3$ & $\mathrm{Y}-\mathrm{H} 2 \mathrm{O}$ & $Y$ \\
\hline 1 & 129,1 & 65,1 & 112,1 & & $\mathrm{~K}$ & 1144,5 & 572,8 & 1127,5 & 1126,5 & 9 \\
\hline 2 & 228.2 & 114,6 & 211,1 & & V & 1016.4 & 508.7 & 999,4 & 998.4 & 8 \\
\hline 3 & 388.2 & 194,6 & 371,2 & & $C+57$ & 917.3 & 459.2 & 900,3 & 899.3 & 7 \\
\hline 4 & 555.2 & 278.1 & 538.2 & 537.2 & $S+80$ & 757.3 & 379.2 & 740.3 & 739.3 & 6 \\
\hline 5 & 656,2 & 328.6 & 639.2 & 638,2 & $\mathbf{T}$ & 590.3 & & 573,3 & 572,3 & 5 \\
\hline 6 & 770.3 & 385.6 & 753.3 & 752,3 & $\mathrm{~N}$ & 489.3 & & 472,2 & 471.3 & 4 \\
\hline 7 & 885.3 & 443.2 & 868.3 & 867.3 & D & 375.2 & & 358,2 & 357.2 & 3 \\
\hline 8 & 998.4 & 499.7 & 981.4 & 980.4 & L & 260.2 & & 243.2 & & 2 \\
\hline 9 & 1144,5 & 572,8 & 1127,5 & 1126,5 & K & 147,1 & & 130,1 & & 1 \\
\hline
\end{tabular}




\section{MS/MS spectrum}

\section{Full-range}

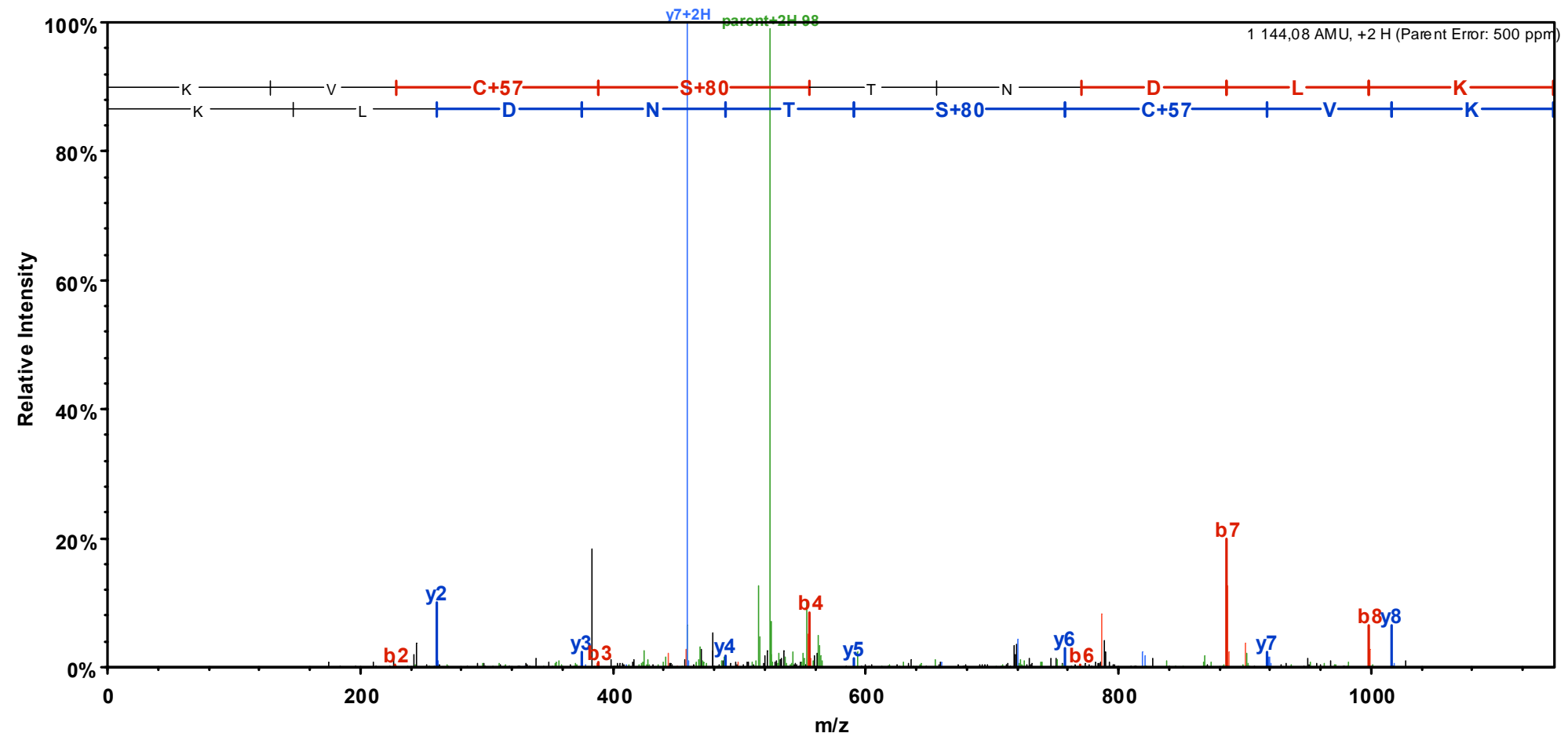

\section{Close to precursor}

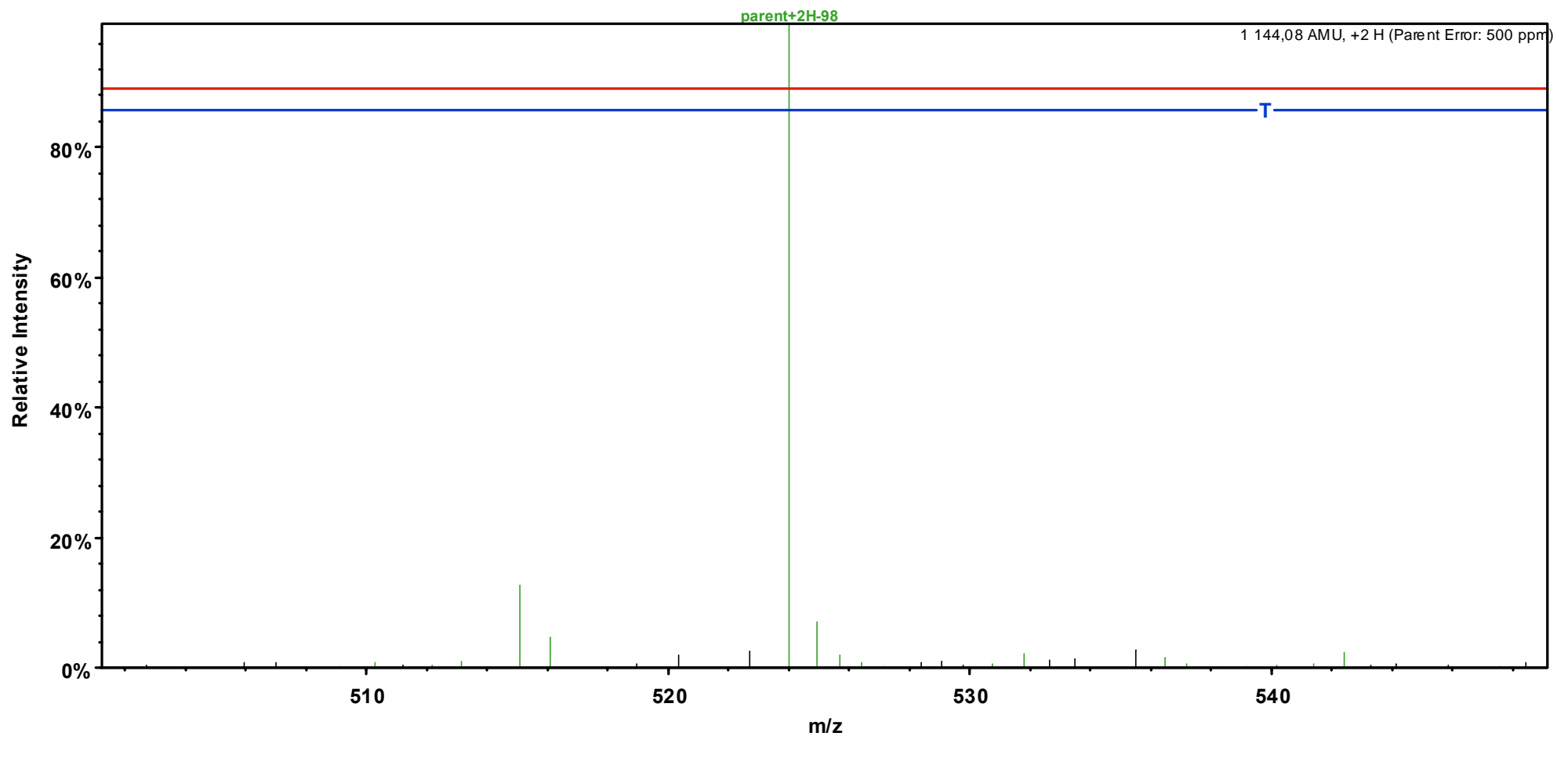




\section{Left of precursor}

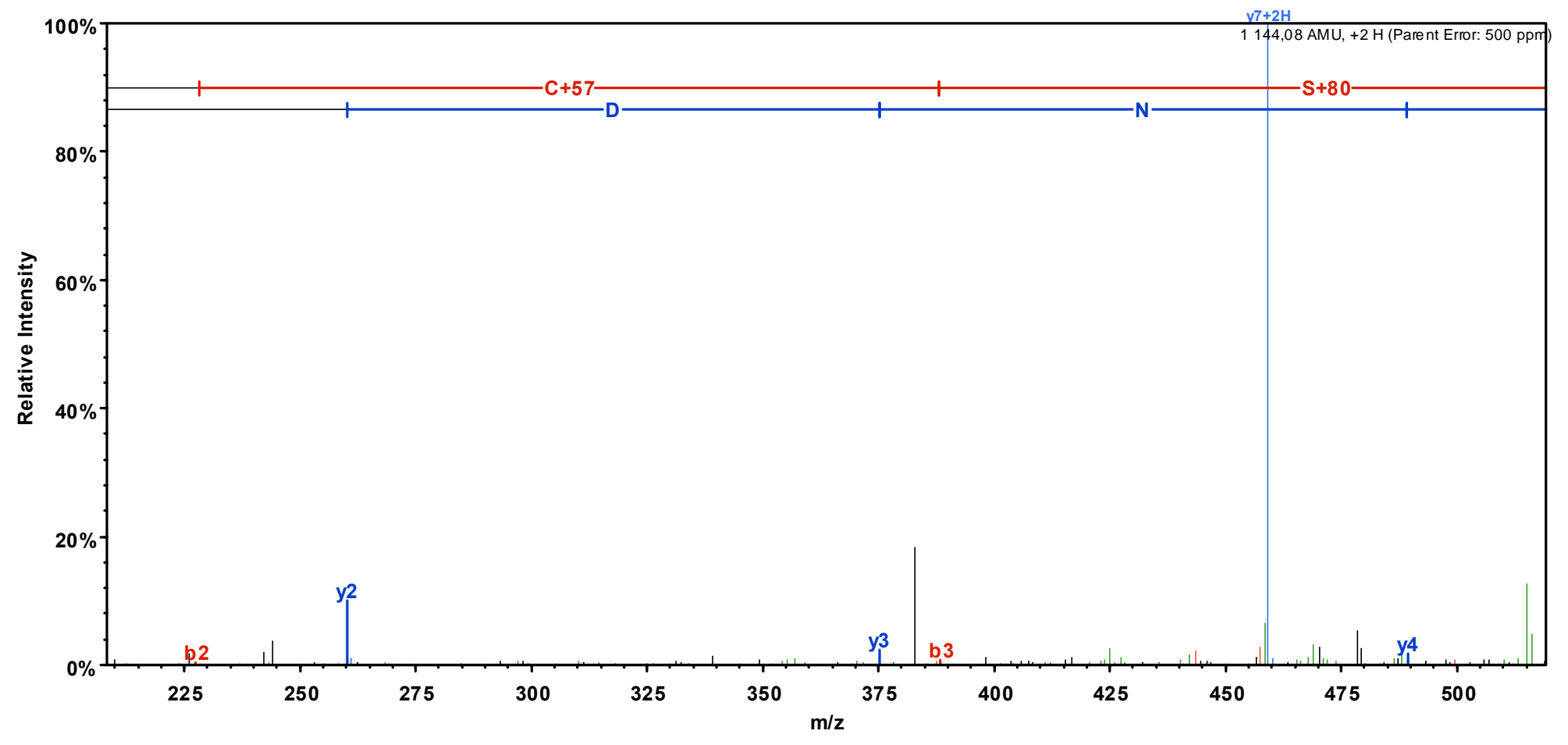

\section{$\underline{\text { Right of precursor }}$}

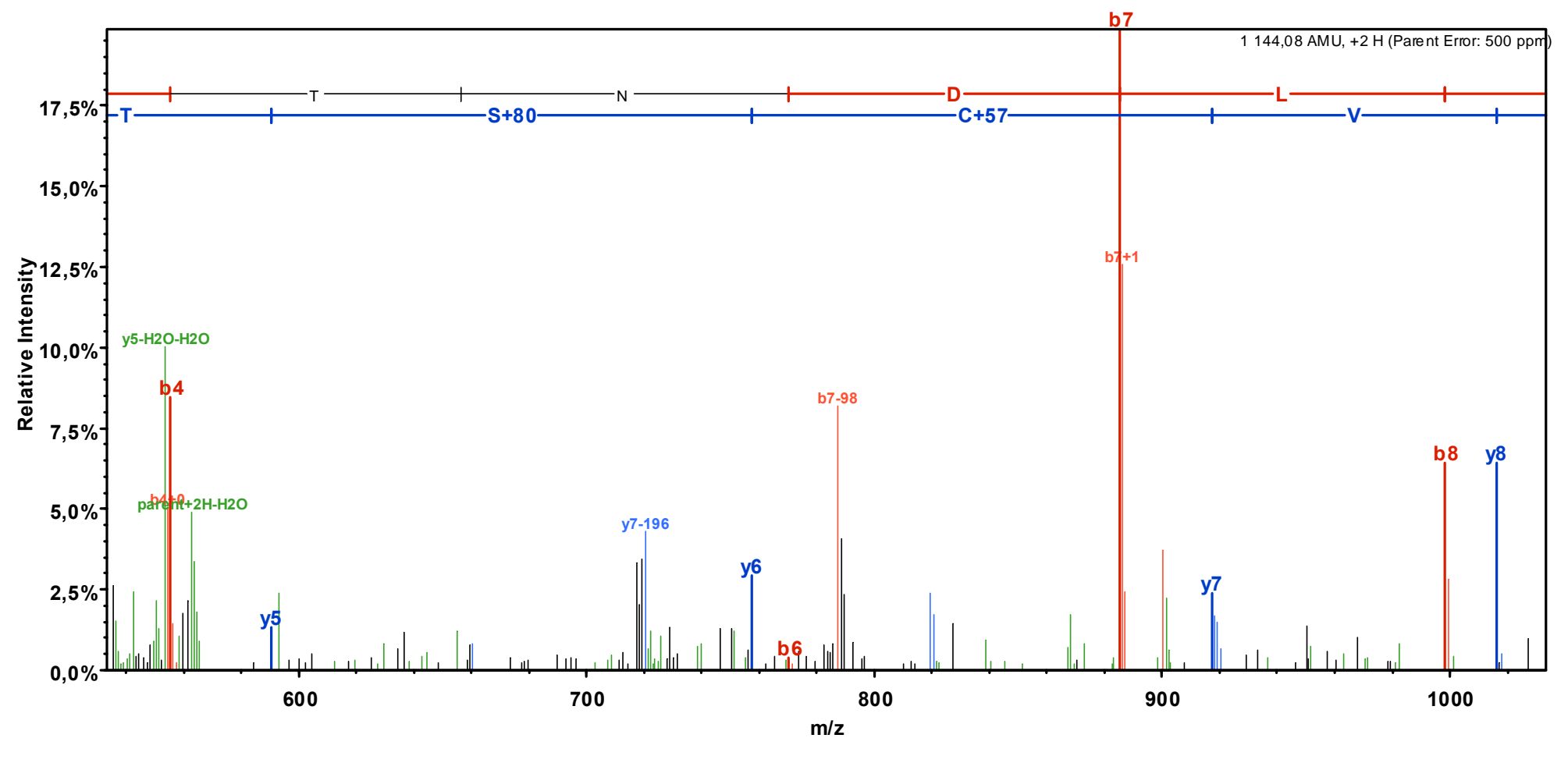




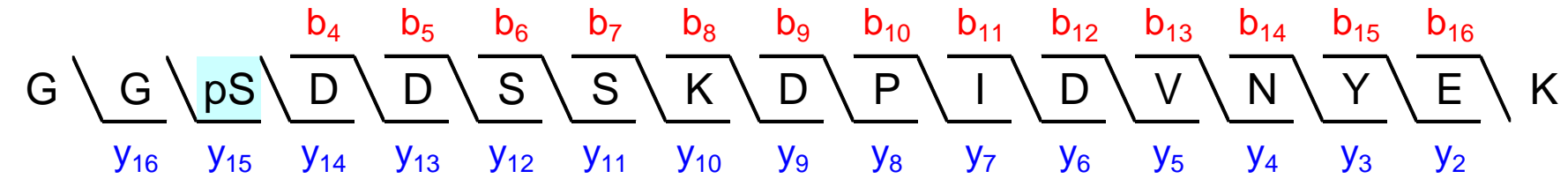

Sequest: XCorr $=5.12, \mathrm{dCn}=0.12$.

Ascore $=23.27$.

Phosphorylation site is localized with $\geq 99 \%$ certainty as Ascore $\geq 20$ (Nat Biotechnol. 2006;24(10):1285-92).

\section{MS/MS spectrum's fragmentation table}

\begin{tabular}{|c|c|c|c|c|c|c|c|c|c|c|}
\hline B & $B$ Ions & $\mathrm{B}+2 \mathrm{H}$ & $\mathrm{B}-\mathrm{NH} 3$ & $\mathrm{~B}-\mathrm{H} 2 \mathrm{O}$ & AA & $Y$ Ions & $\mathrm{Y}+2 \mathrm{H}$ & $\mathrm{Y}-\mathrm{NH} 3$ & $\mathrm{Y}-\mathrm{H} 2 \mathrm{O}$ & $\mathrm{Y}$ \\
\hline 1 & 58,0 & & & & G & 1905,8 & 953.4 & 1888,7 & 1887.8 & 17 \\
\hline 2 & 115,1 & & & & G & 1848,8 & 924.9 & 1831,7 & 1830.7 & 16 \\
\hline 3 & 282,0 & & & 264,0 & S+80 & 1791,7 & 896.4 & 1774,7 & 1773,7 & 15 \\
\hline 4 & 397.1 & & & 379,1 & D & 1624,7 & 812,9 & 1607,7 & 1606,7 & 14 \\
\hline 5 & 512.1 & & & 494,1 & D & 1509.7 & 755.4 & 1492.7 & 1491,7 & 13 \\
\hline 6 & 599.1 & 300,1 & & 581,1 & S & 1394.7 & 697.8 & 1377.7 & 1376.7 & 12 \\
\hline 7 & 686.2 & 343,6 & & 668.2 & S & 1307.6 & 654,3 & 1290,6 & 1289.6 & 11 \\
\hline 8 & 814.3 & 407.6 & 797,2 & 796.3 & K & 1220.6 & 610,8 & 1203.6 & 1202,6 & 10 \\
\hline 9 & 929.3 & 465,1 & 912,3 & 911.3 & D & 1092.5 & 546,8 & 1075.5 & 1074.5 & 9 \\
\hline 10 & 1026.3 & 513.7 & 1009.3 & 1008,3 & $\mathbf{P}$ & 977.5 & 489,3 & 960.5 & 959,5 & 8 \\
\hline 11 & 1139.4 & 570,2 & 1122,4 & 1121.4 & I & 880.4 & 440.7 & 863.4 & 862,4 & 7 \\
\hline 12 & 1254.5 & 627,7 & 1237.4 & 1236.4 & D & 767.4 & 384,2 & 750.3 & 749,3 & 6 \\
\hline 13 & 1353.5 & 677,3 & 1336.5 & 1335.5 & V & 652.3 & & 635.3 & 634.3 & 5 \\
\hline 14 & 1467.6 & 734.3 & 1450.5 & 1449.6 & $\mathrm{~N}$ & 553.3 & & 536.2 & 535.3 & 4 \\
\hline 15 & 1630.6 & 815.8 & 1613.6 & 1612,6 & $\mathbf{Y}$ & 439.2 & & 422.2 & 421,2 & 3 \\
\hline 16 & 1759.7 & 880.3 & 1742,6 & 1741,7 & E & 276.2 & & 259,1 & 258,1 & 2 \\
\hline 17 & 1905,8 & 953.4 & 1888.7 & 1887,8 & K & 147,1 & & 130,1 & & 1 \\
\hline
\end{tabular}




\section{MS/MS spectrum}

\section{Full-range}

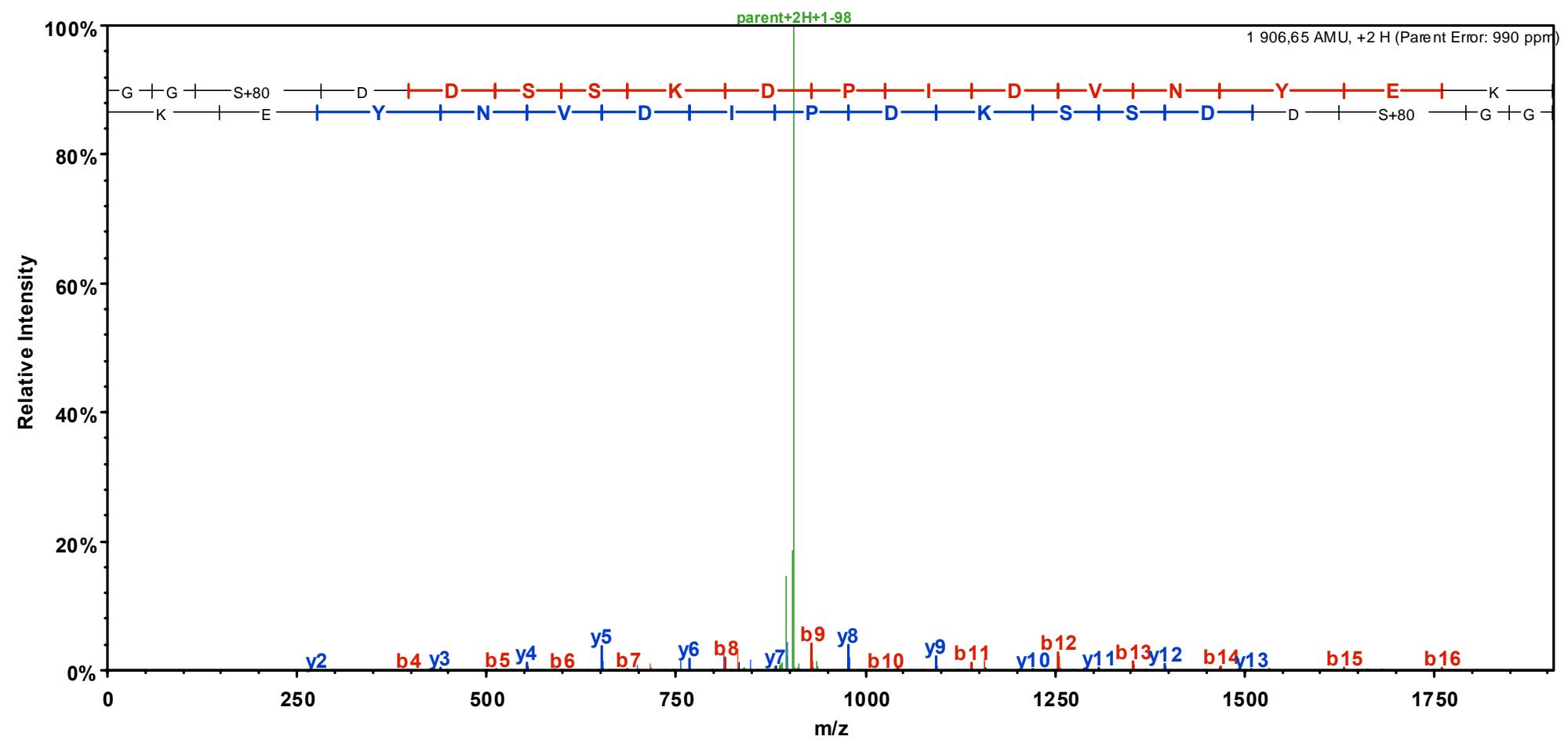

\section{Close to precursor}

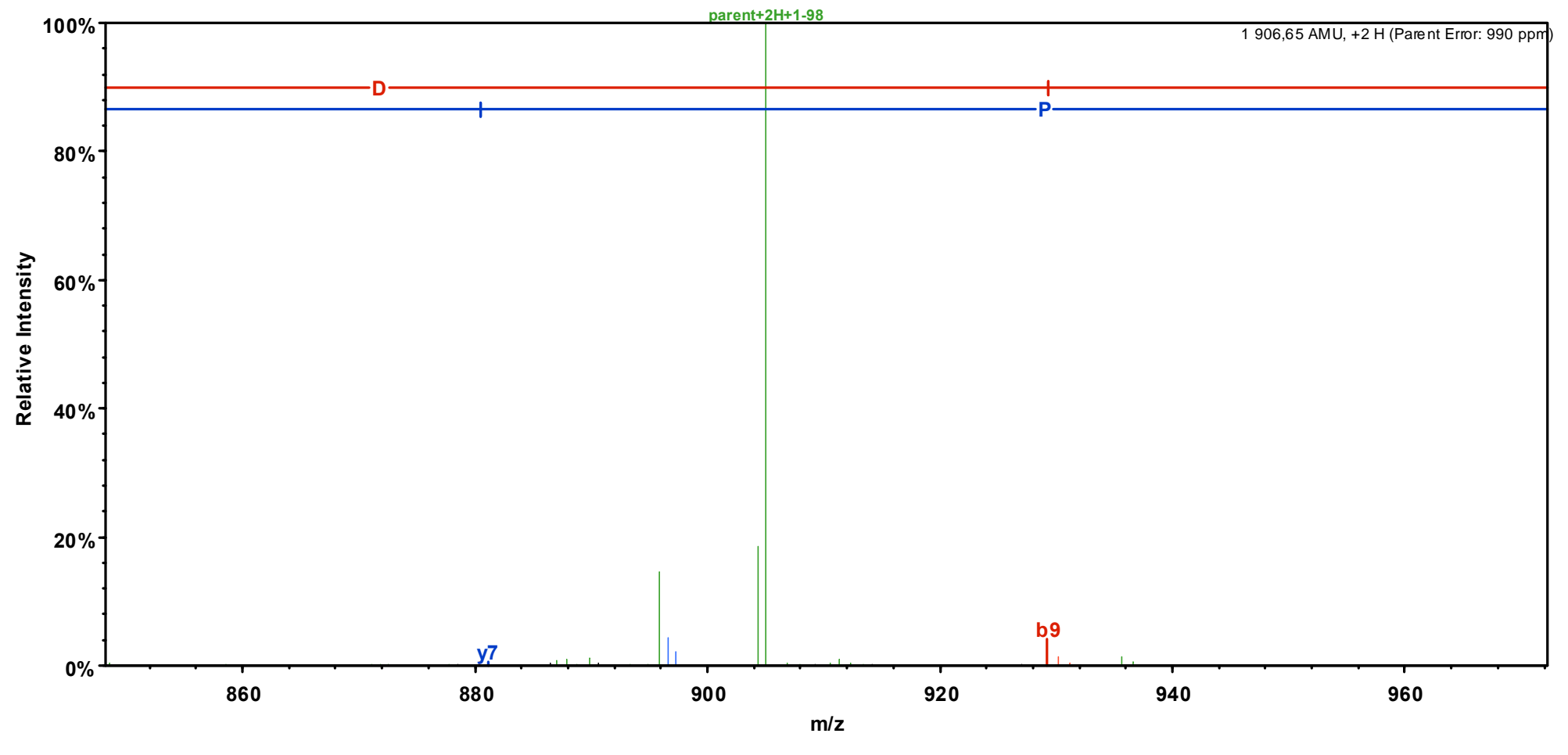




\section{$\underline{\text { Left of precursor }}$}

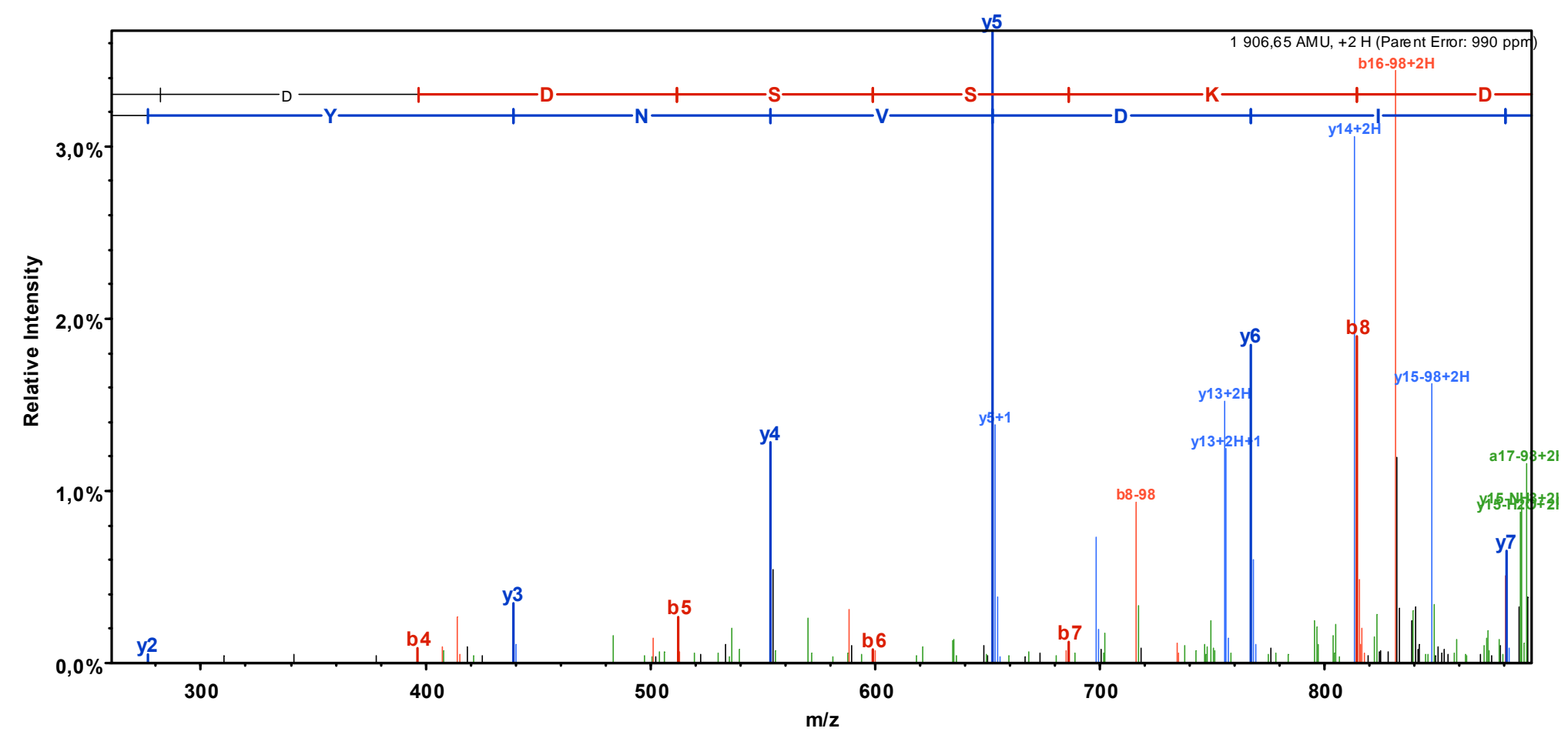

\section{$\underline{\text { Right of precursor }}$}

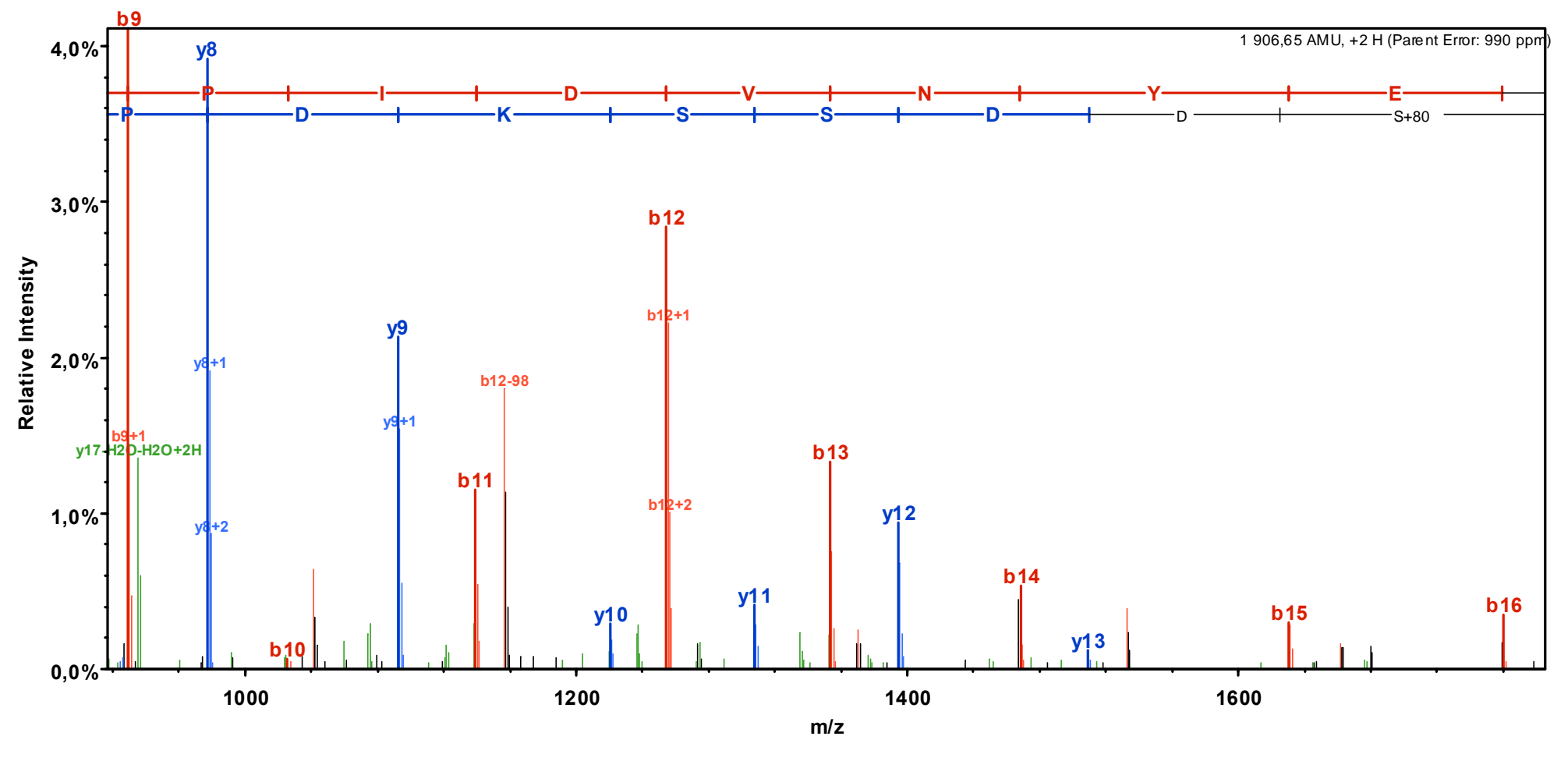




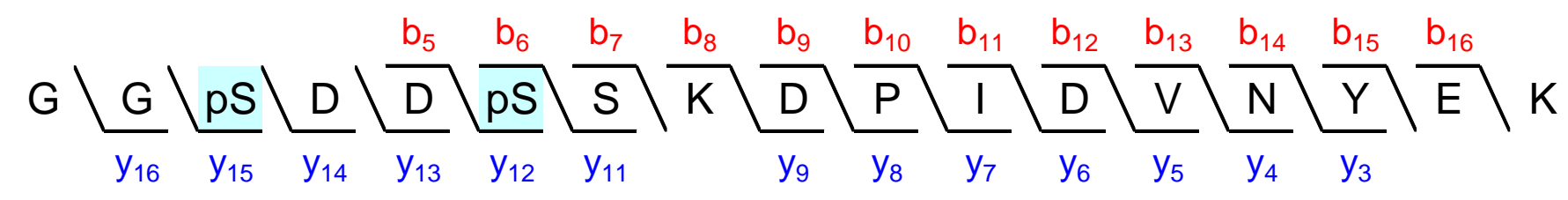

Sequest: $X$ Corr $=5.04, \mathrm{dCn} 1=0.05$ (between $1^{\text {st }}$ and $2^{\text {nd }}$ best matches), $\mathrm{dCn} 2=0.13$ (between $2^{\text {nd }}$ and $3^{\text {rd }}$ best matches). $A_{\text {score }}=28.34$ (first phosphorylation), 11.51 (second phosphorylation).

Sequest's second best match with XCorr $=4.79$ is to the same peptide sequence but with an alternative phosphorylation site: GGpSDDSpSKDPIDVNYEK.

The weak Ascore for the second phosphorylation indicates that, given the observed MS/MS spectrum, the phosphopeptide identified could either be Sequest's first or second best match (Nat Biotechnol. 2006;24(10):1285-92).

\section{MS/MS spectrum's fragmentation table}

\begin{tabular}{|c|c|c|c|c|c|c|c|c|c|c|}
\hline B & B Ions & $\mathrm{B}+2 \mathrm{H}$ & $\mathrm{B}-\mathrm{NH} 3$ & $\mathrm{~B}-\mathrm{H} 2 \mathrm{O}$ & AA & $Y$ Ions & $\mathrm{Y}+2 \mathrm{H}$ & $\mathrm{Y}-\mathrm{NH} 3$ & $\mathrm{Y}-\mathrm{H} 2 \mathrm{O}$ & $Y$ \\
\hline 1 & 58,0 & & & & G & 1985,7 & 993,4 & 1968.7 & 1967.7 & 17 \\
\hline 2 & 115,1 & & & & G & 1928.7 & 964.9 & 1911,7 & 1910,7 & 16 \\
\hline 3 & 282,0 & & & 264,0 & $S+80$ & 1871,7 & 936.4 & 1854,7 & 1853,7 & 15 \\
\hline 4 & 397,1 & & & 379,1 & D & 1704.7 & 852.9 & 1687,7 & 1686.7 & 14 \\
\hline 5 & 512.1 & & & 494,1 & D & 1589,7 & 795.3 & 1572,6 & 1571,7 & 13 \\
\hline 6 & 679.1 & 340,1 & & 661.1 & $\mathbf{S + 8 0}$ & 1474.6 & 737,8 & 1457.6 & 1456.6 & 12 \\
\hline 7 & 766.1 & 383.6 & & 748.1 & S & 1307.6 & 654,3 & 1290.6 & 1289,6 & 11 \\
\hline 8 & 894.2 & 447,6 & 877.2 & 876.2 & K & 1220,6 & 610,8 & 1203,6 & 1202,6 & 10 \\
\hline 9 & 1009.3 & 505,1 & 992,2 & 991,2 & D & 1092.5 & 546.8 & 1075.5 & 1074.5 & 9 \\
\hline 10 & 1106,3 & 553,7 & 1089.3 & 1088,3 & $\mathbf{P}$ & 977.5 & 489,3 & 960.5 & 959.5 & 8 \\
\hline 11 & 1219.4 & 610,2 & 1202.4 & 1201.4 & I & 880.4 & 440,7 & 863.4 & 862,4 & 7 \\
\hline 12 & 1334.4 & 667.7 & 1317.4 & 1316.4 & D & 767,4 & 384,2 & 750.3 & 749,3 & 6 \\
\hline 13 & 1433.5 & 717,2 & 1416.5 & 1415.5 & V & 652.3 & & 635.3 & 634,3 & 5 \\
\hline 14 & 1547.5 & 774.3 & 1530.5 & 1529.5 & $\mathrm{~N}$ & 553.3 & & 536.2 & 535.3 & 4 \\
\hline 15 & 1710.6 & 855.8 & 1693.6 & 1692,6 & $\mathbf{Y}$ & 439.2 & & 422,2 & 421.2 & 3 \\
\hline 16 & 1839.6 & 920.3 & 1822,6 & 1821,6 & $\mathrm{E}$ & 276,2 & & 259,1 & 258,1 & 2 \\
\hline 17 & 1985,7 & 993,4 & 1968,7 & 1967.7 & K & 147,1 & & 130,1 & & 1 \\
\hline
\end{tabular}




\section{MS/MS spectrum}

\section{Full-range}

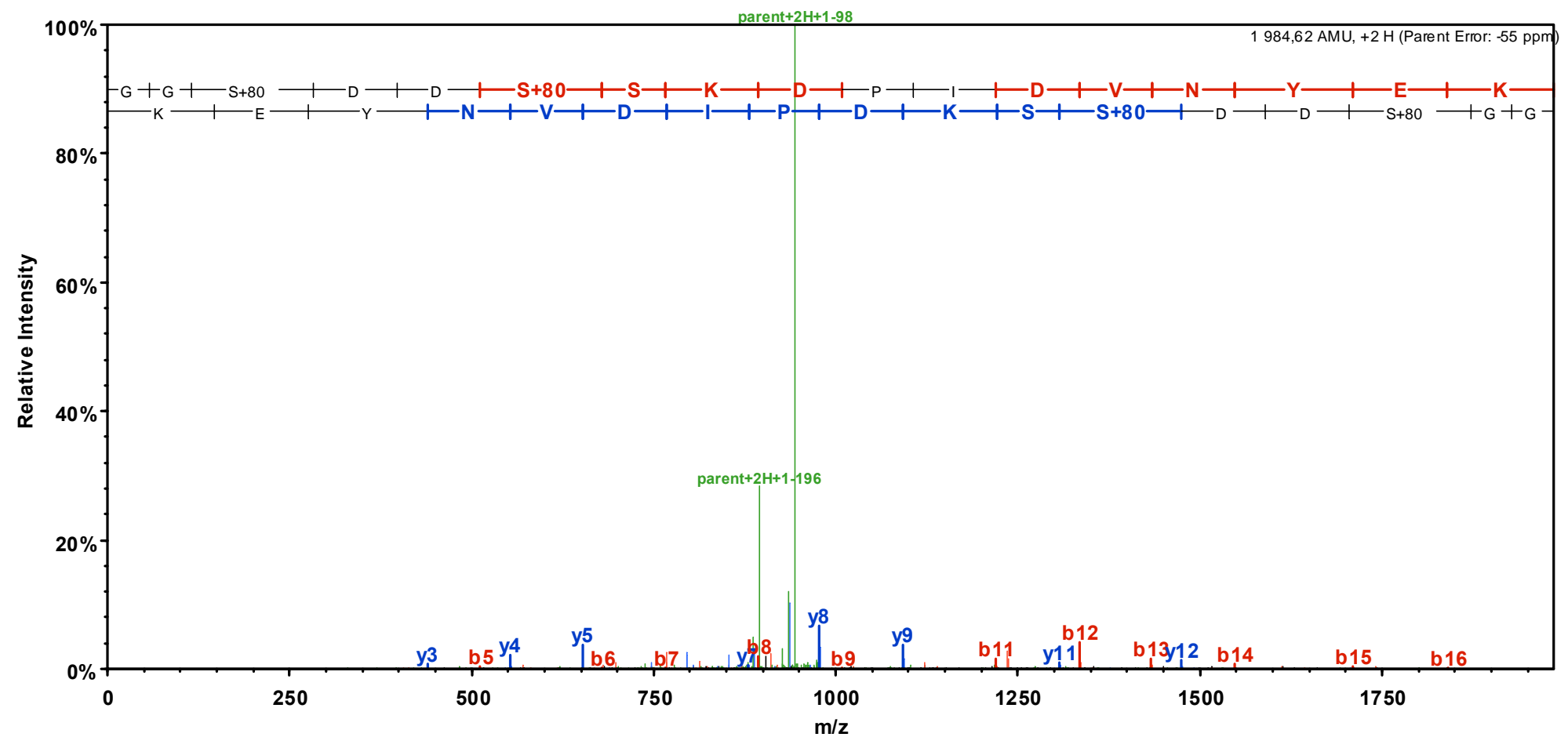

\section{Close to precursor}

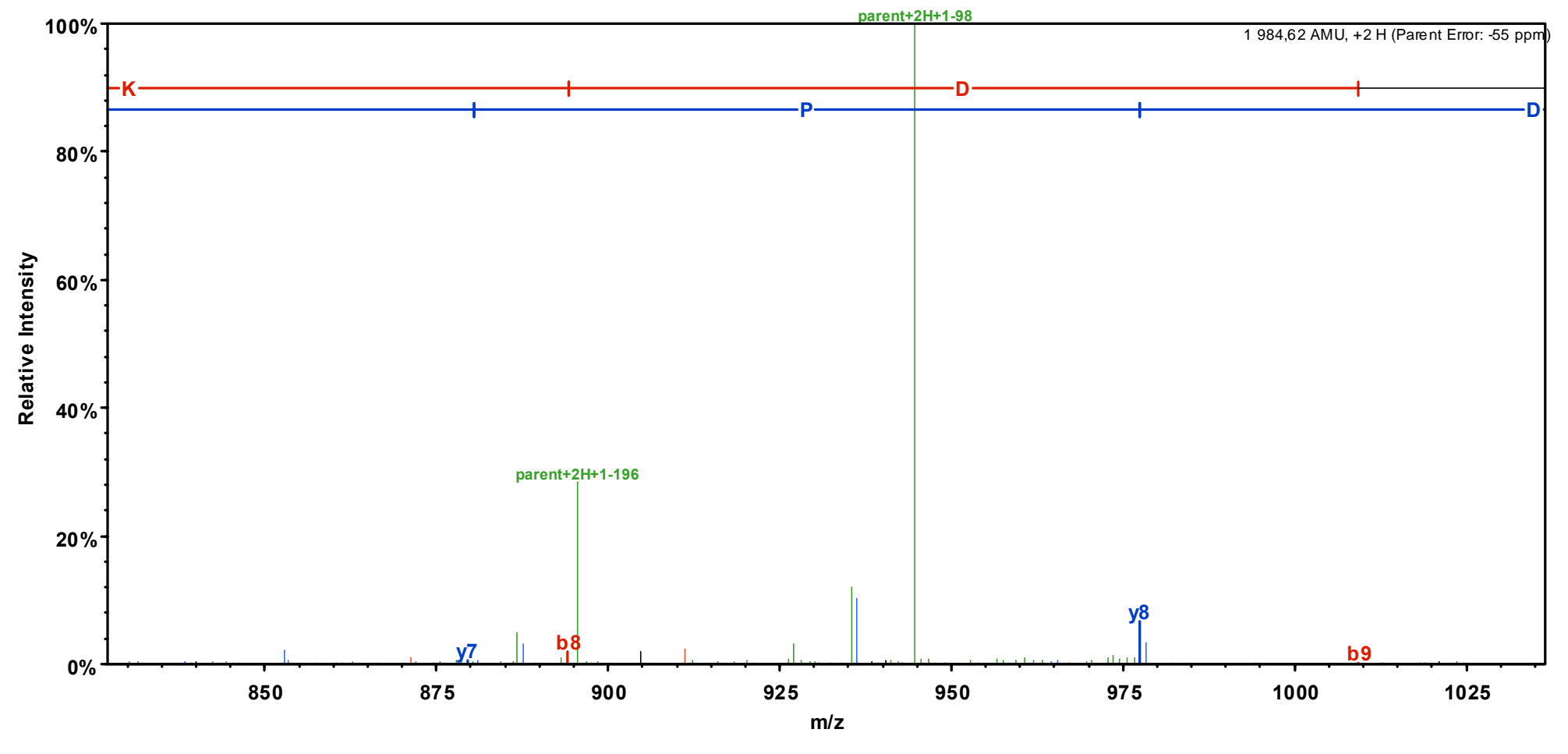




\section{Left of precursor}

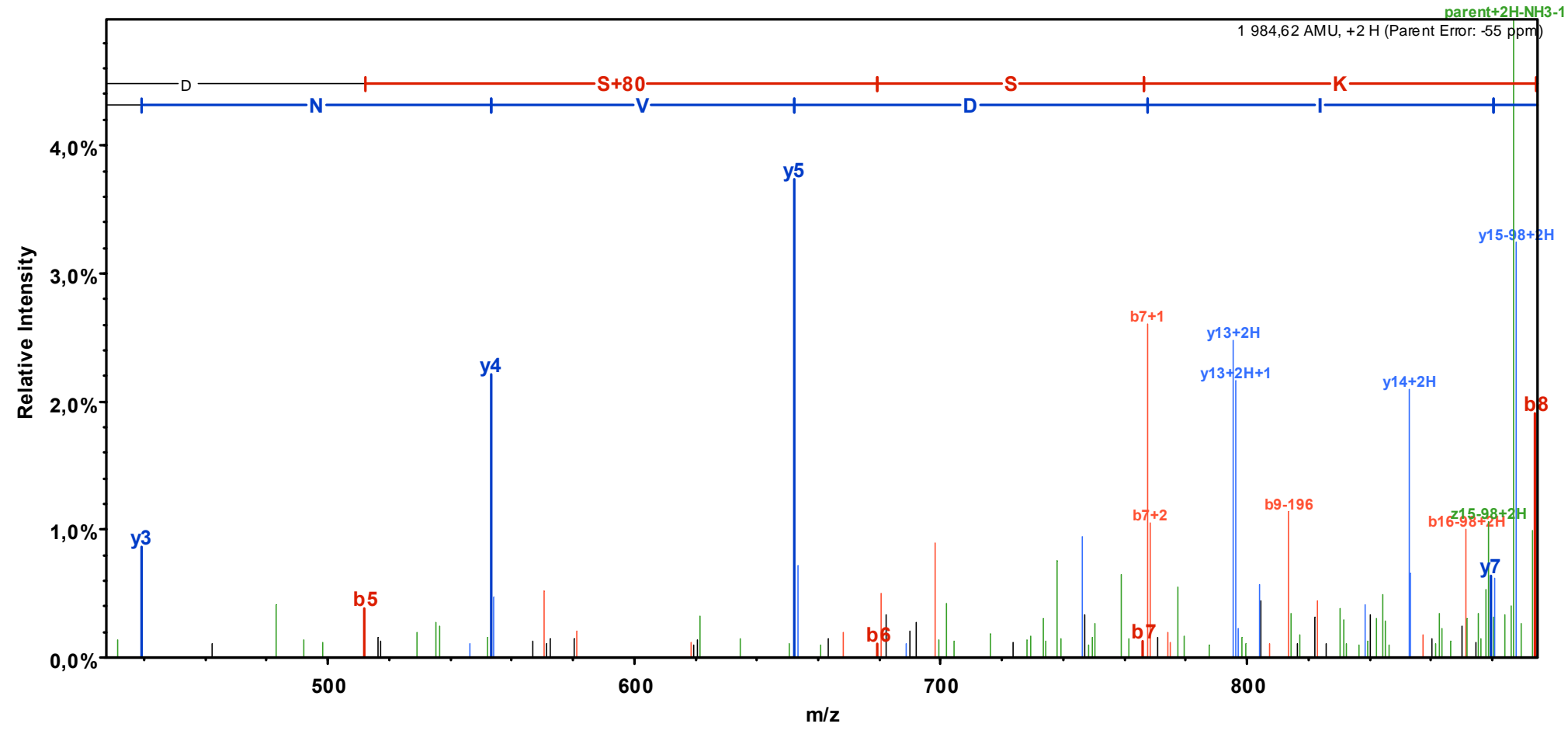

\section{$\underline{\text { Right of precursor }}$}

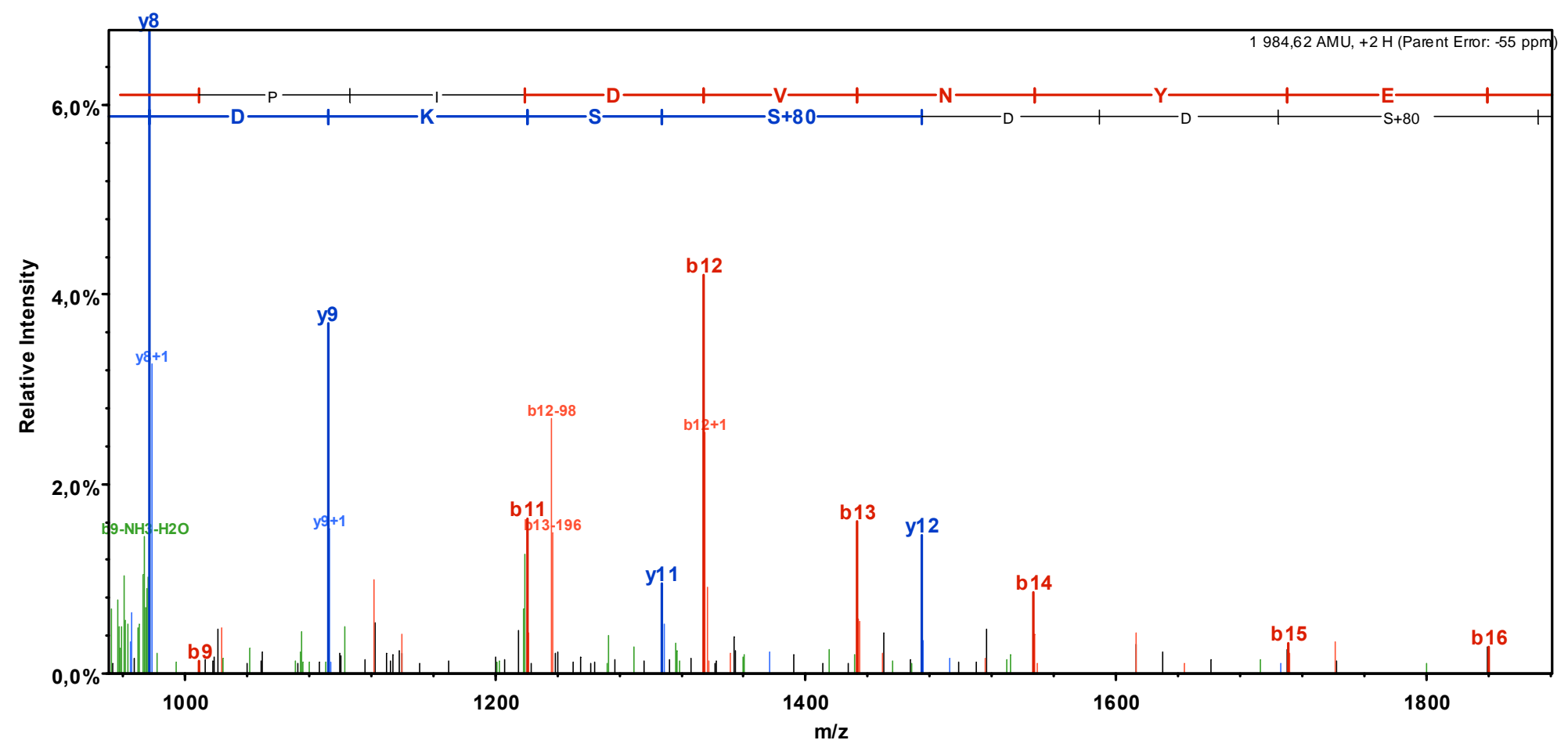


Supplementary mass spectrometric data:

PARP-1,

phosphorylated with C-Jun N-Terminal Kinase 1 (JNK1)

Spectrum Ingel8474.12185.12185.3.dta (charge state: +3)

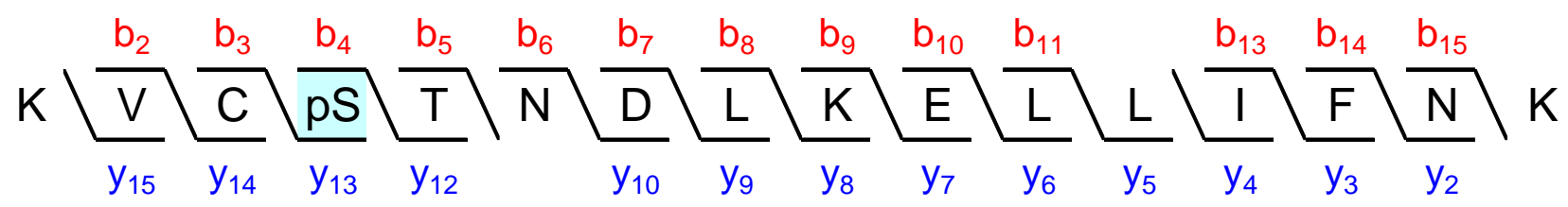

Sequest: $X$ Corr $=5.29, \mathrm{dCn} 1=0.06$ (between $1^{\text {st }}$ and $2^{\text {nd }}$ best matches), $\mathrm{dCn} 2=0.85$ (between $2^{\text {nd }}$ and $3^{\text {rd }}$ best matches). $\mathrm{A}_{\text {score }}=13.49$.

Sequest's second best matches with XCorr $=4.97$ is to the same peptide sequence but with an alternative phosphorylation site:

KVCSPTNDLKELLIFNK

The weak Ascore indicates that, given the observed MS/MS spectrum, the phosphopeptide identified could either be Sequest's first or second best match (Nat Biotechnol. 2006;24(10):1285-92).

\section{MS/MS spectrum's fragmentation table}

\begin{tabular}{|c|c|c|c|c|c|c|c|c|c|c|}
\hline B & B Ions & $\mathrm{B}+2 \mathrm{H}$ & $\mathrm{B}-\mathrm{NH} 3$ & $\mathrm{~B}-\mathrm{H} 2 \mathrm{O}$ & AA & $Y$ Ions & $\mathrm{Y}+2 \mathrm{H}$ & $\mathrm{Y}-\mathrm{NH} 3$ & $\mathrm{Y}-\mathrm{H} 2 \mathrm{O}$ & $Y$ \\
\hline 1 & 129,1 & 65,1 & 112,1 & & $\mathrm{~K}$ & 2002,0 & 1001,5 & 1985,0 & 1984,0 & 16 \\
\hline 2 & 228.2 & 114,6 & 211,1 & & V & 1873,9 & 937.5 & 1856,9 & 1855,9 & 15 \\
\hline 3 & 388.2 & 194,6 & 371,2 & & $C+57$ & 1774,8 & 887.9 & 1757.8 & 1756,8 & 14 \\
\hline 4 & 555.2 & 278,1 & 538.2 & 537,2 & $S+80$ & 1614,8 & 807.9 & 1597,8 & 1596,8 & 13 \\
\hline 5 & 656.2 & 328,6 & 639.2 & 638.2 & $\mathbf{T}$ & 1447,8 & 724.4 & 1430,8 & 1429,8 & 12 \\
\hline 6 & 770.3 & 385,6 & 753.3 & 752,3 & $\mathrm{~N}$ & 1346,8 & 673.9 & 1329,7 & 1328,8 & 11 \\
\hline 7 & 885.3 & 443,2 & 868.3 & 867,3 & D & 1232,7 & 616.9 & 1215,7 & 1214,7 & 10 \\
\hline 8 & 998.4 & 499,7 & 981,4 & 980,4 & L & 1117.7 & 559.4 & 1100,7 & 1099.7 & 9 \\
\hline 9 & 1126.5 & 563.8 & 1109,5 & 1108.5 & K & 1004.6 & 502.8 & 987.6 & 986.6 & 8 \\
\hline 10 & 1255.5 & 628,3 & 1238.5 & 1237,5 & $E$ & 876.5 & 438.8 & 859.5 & 858,5 & 7 \\
\hline 11 & 1368,6 & 684.8 & 1351,6 & 1350,6 & L & 747.5 & 374.2 & 730,5 & & 6 \\
\hline 12 & 1481,7 & 741,4 & 1464,7 & 1463,7 & L & 634,4 & & 617.4 & & 5 \\
\hline 13 & 1594,8 & 797.9 & 1577.8 & 1576,8 & I & 521.3 & & 504.3 & & 4 \\
\hline 14 & 1741,9 & 871.4 & 1724,8 & 1723.8 & $\mathbf{F}$ & 408.2 & & 391.2 & & 3 \\
\hline 15 & 1855,9 & 928.5 & 1838,9 & 1837,9 & $\mathrm{~N}$ & 261.2 & & 244.1 & & 2 \\
\hline 16 & 2002,0 & 1001,5 & 1985,0 & 1984,0 & K & 147,1 & & 130,1 & & 1 \\
\hline
\end{tabular}




\section{MS/MS spectrum}

\section{Full-range}

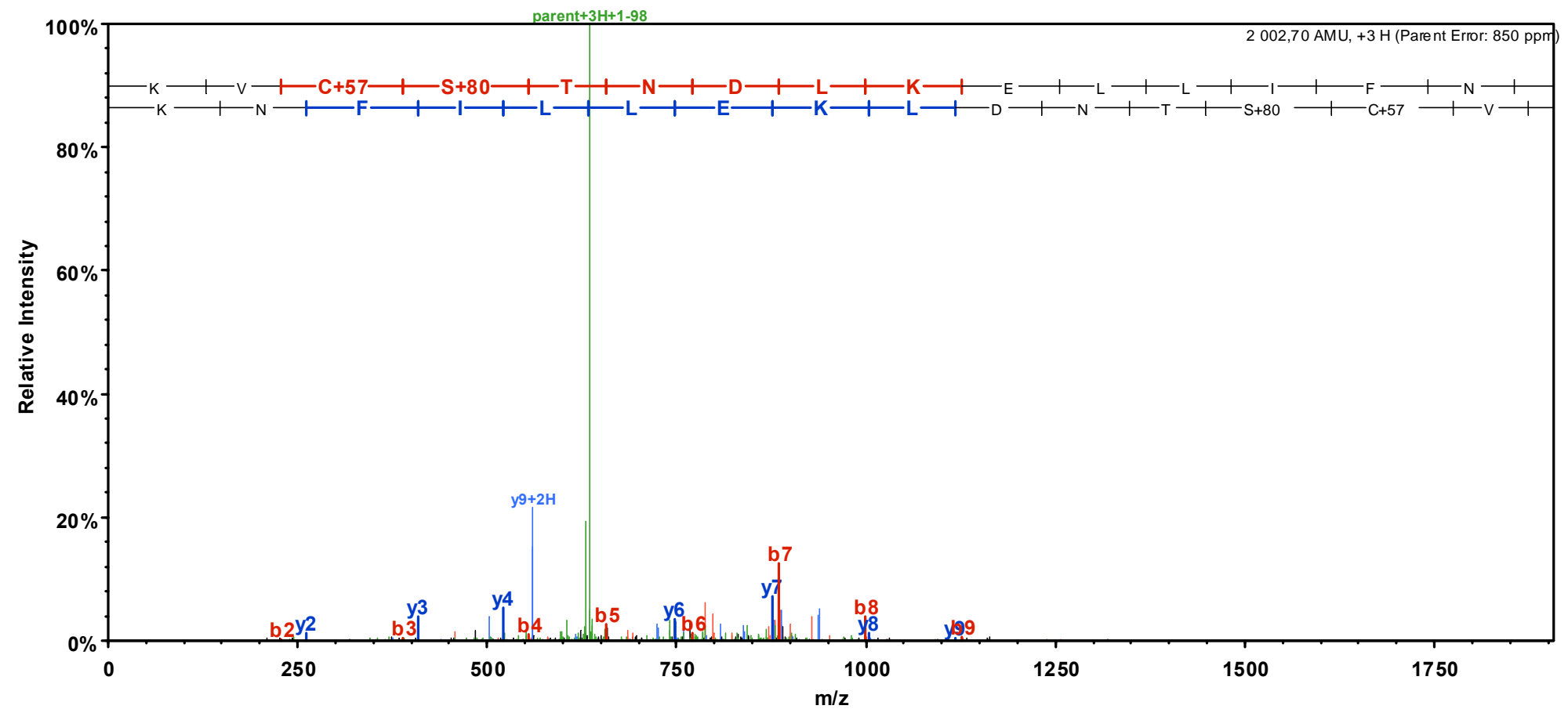

\section{$\underline{\text { Close to precursor }}$}

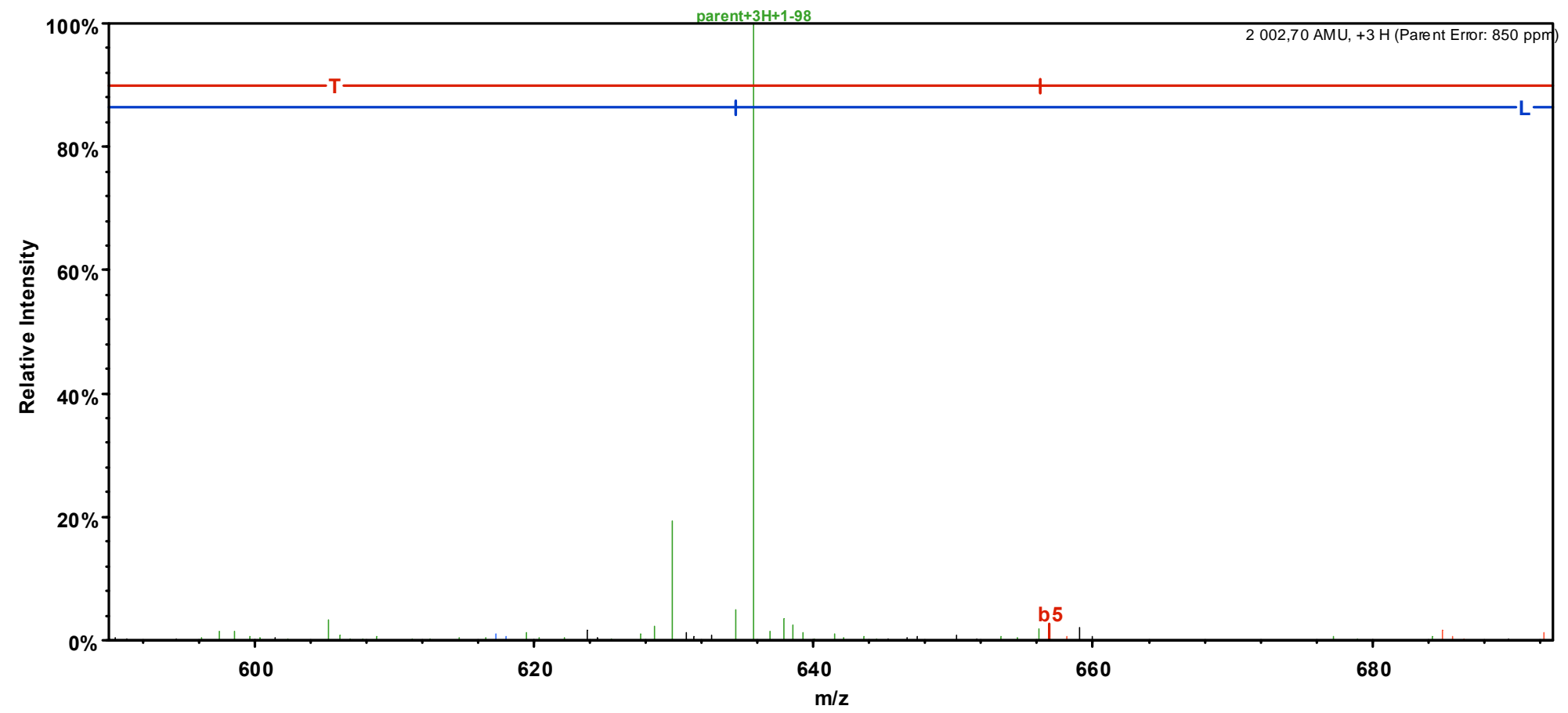




\section{Left of precursor}

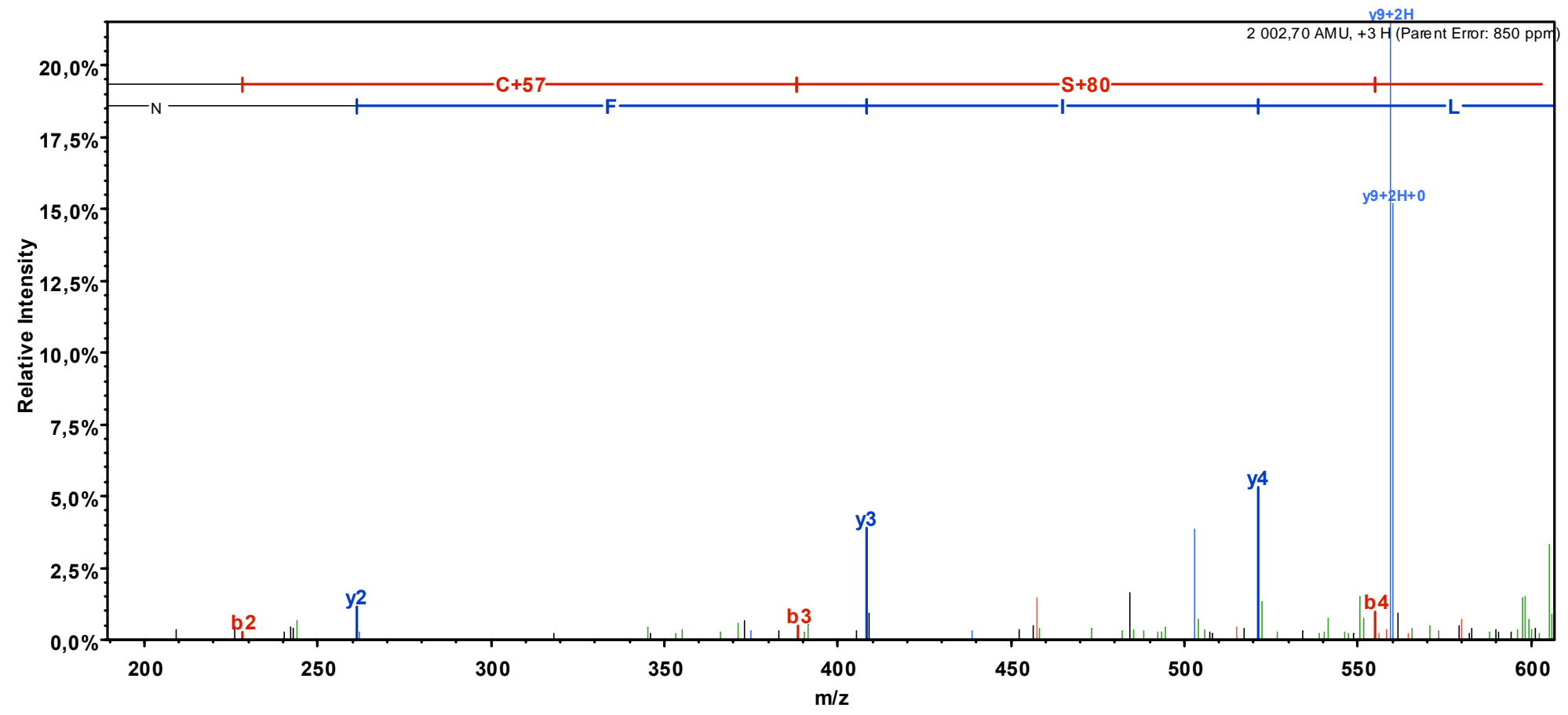

\section{$\underline{\text { Right of precursor }}$}

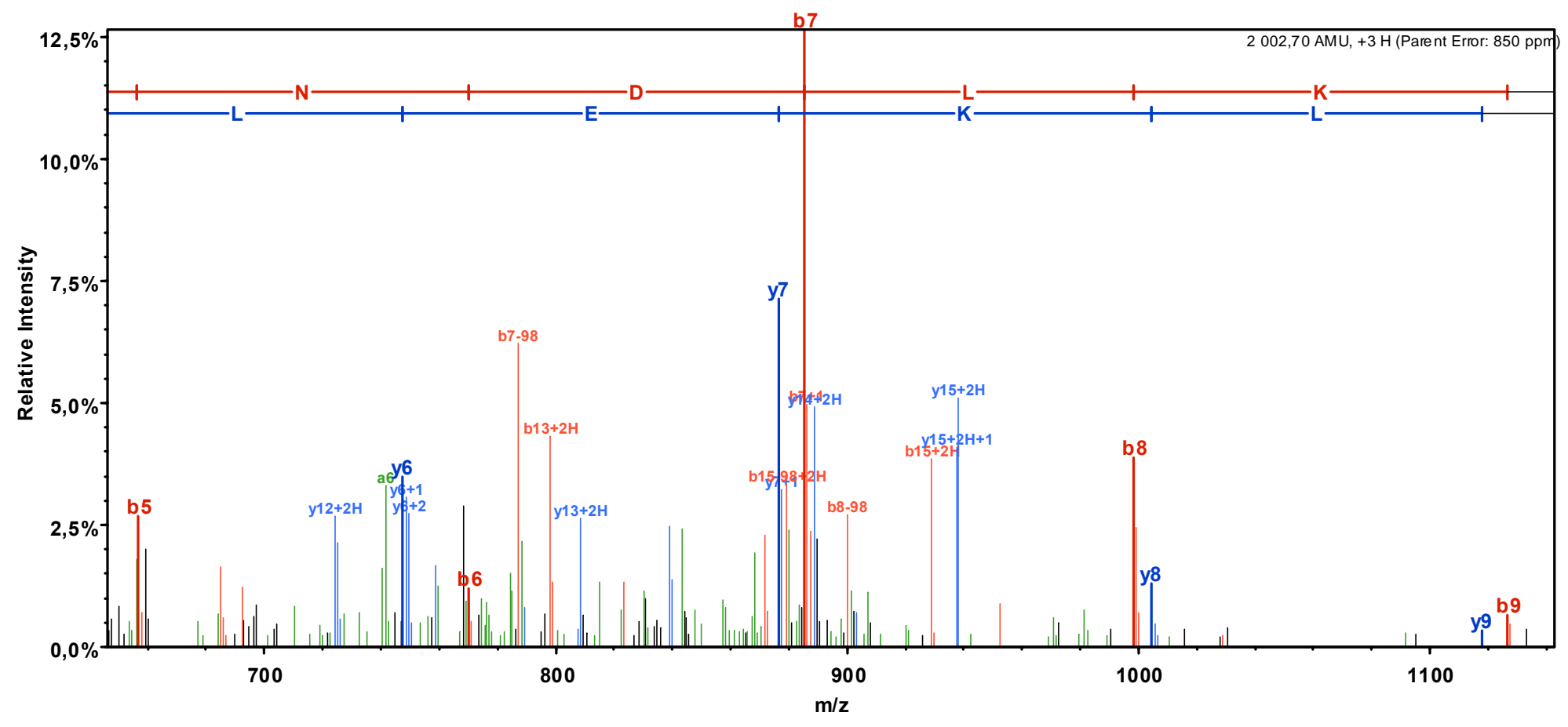




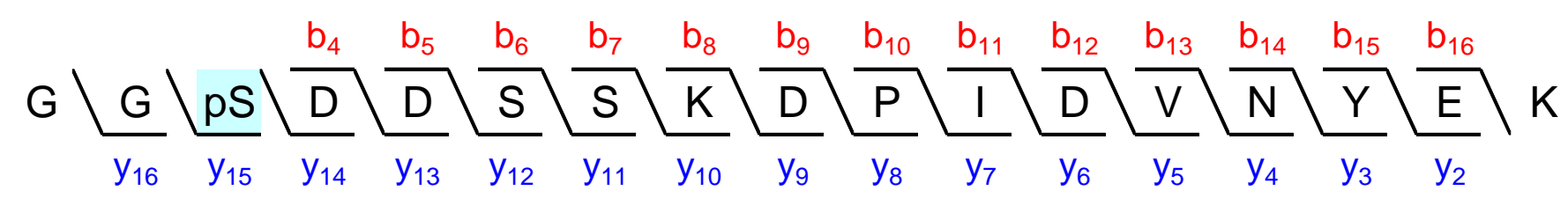

Sequest: XCorr $=5.43, d C n=0.99$.

Ascore $=32.37$.

Phosphorylation site is localized with $\geq 99 \%$ certainty as Ascore $\geq 20$ (Nat Biotechnol. 2006;24(10):1285-92).

\section{MS/MS spectrum's fragmentation table}

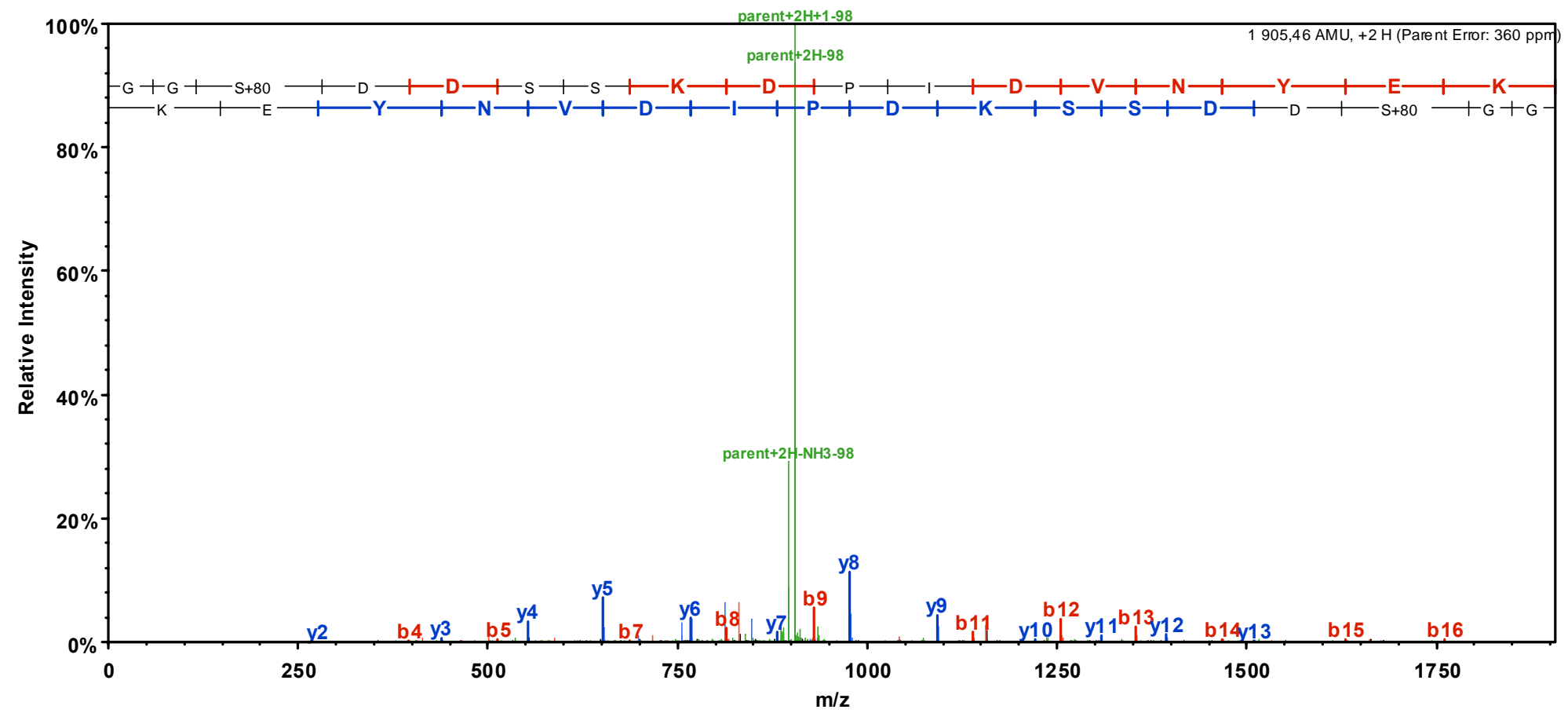




\section{MS/MS spectrum}

\section{Full-range}

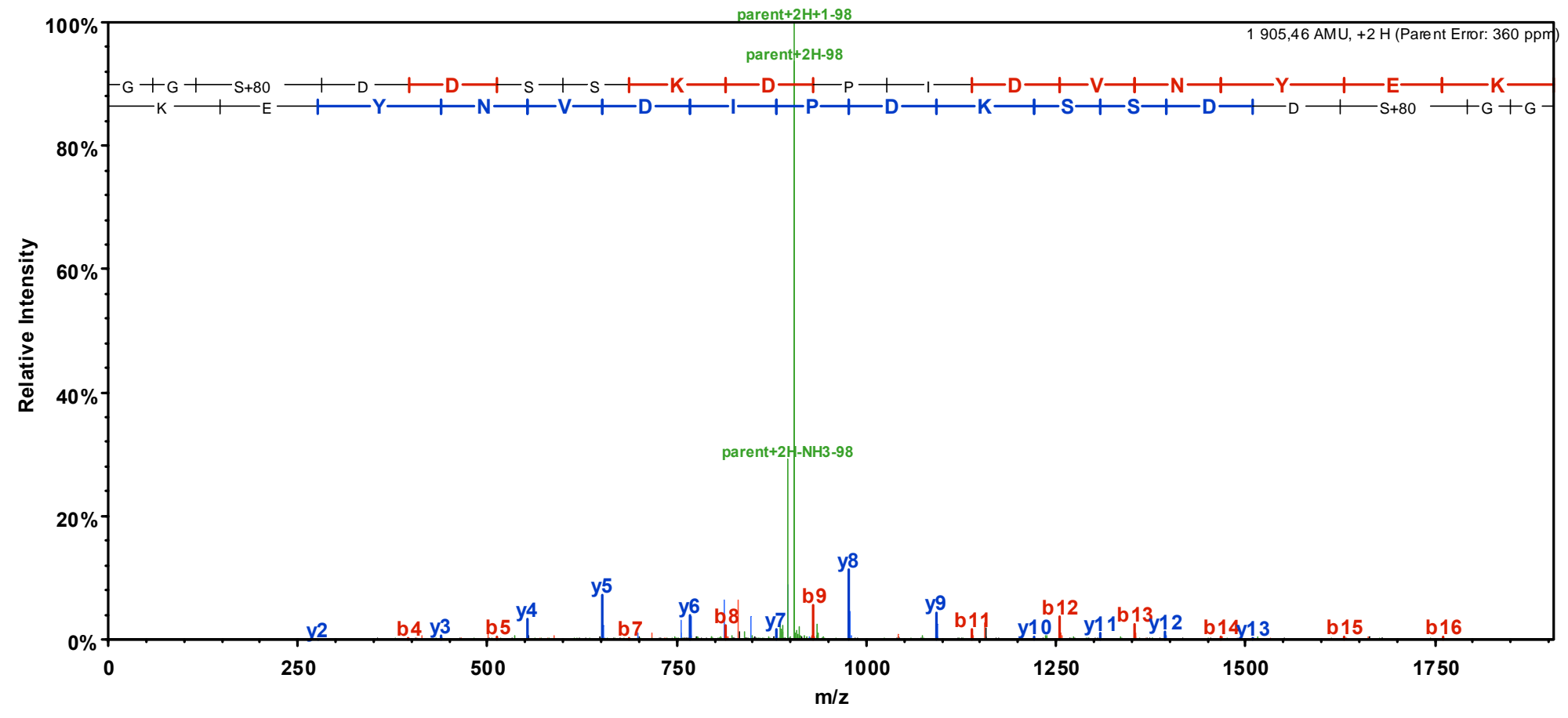

\section{$\underline{\text { Close to precursor }}$}

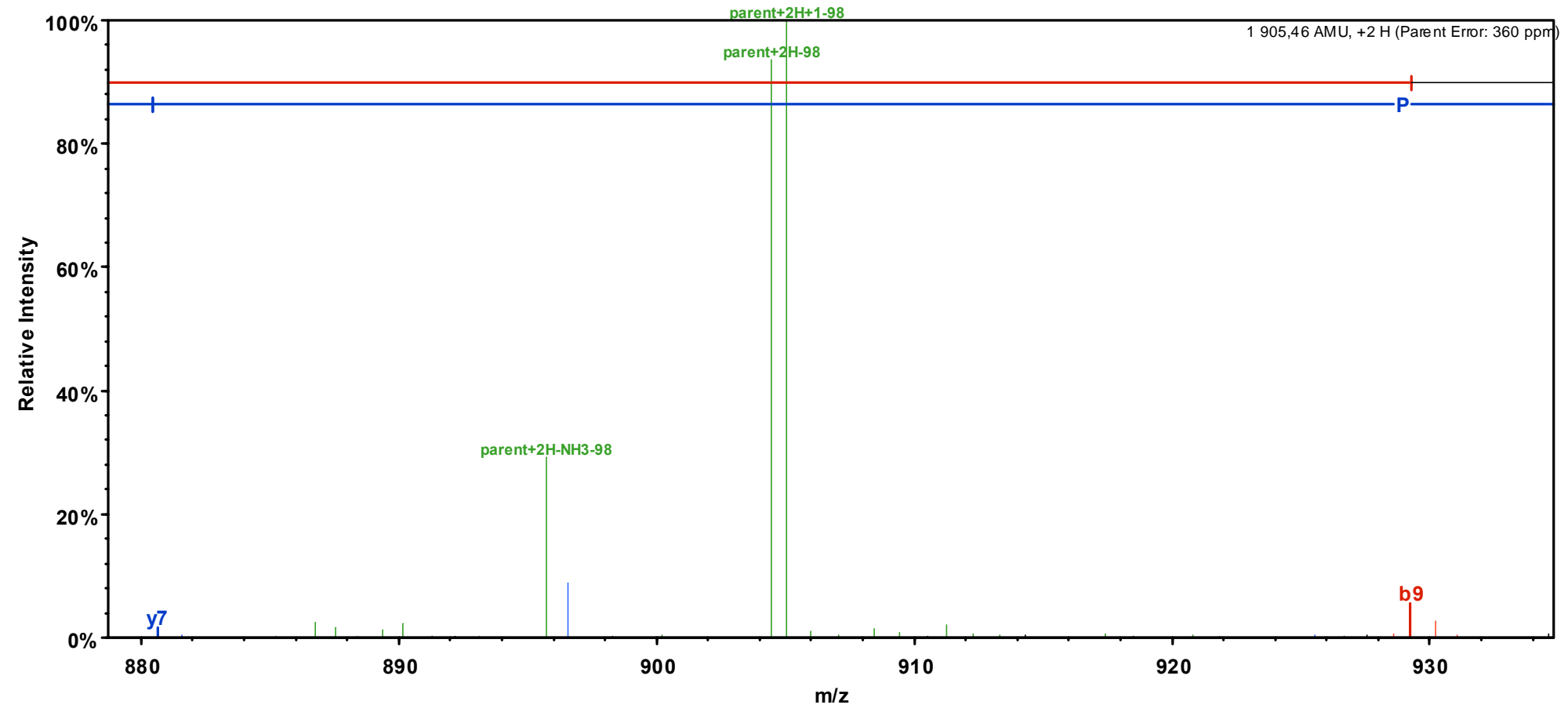




\section{$\underline{\text { Left of precursor }}$}

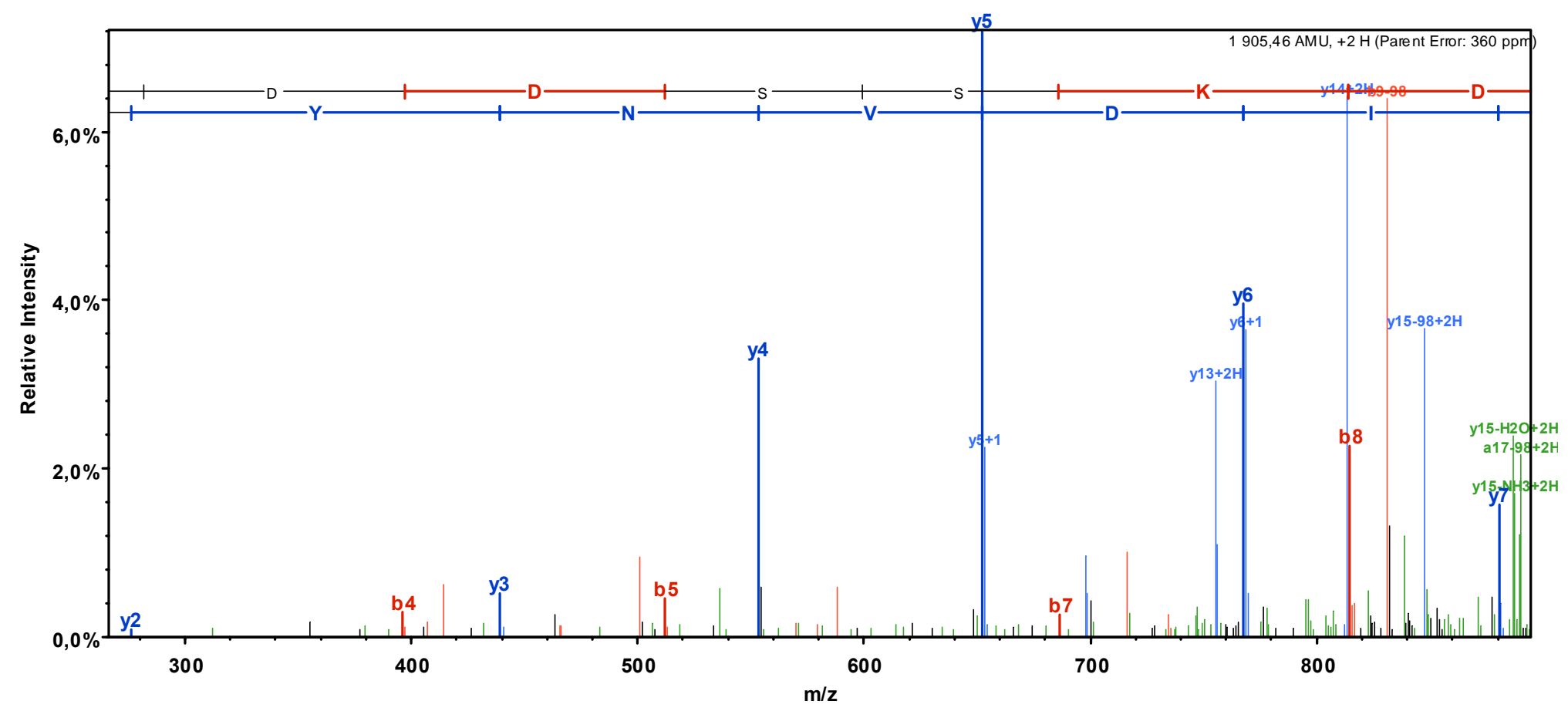

\section{$\underline{\text { Right of precursor }}$}

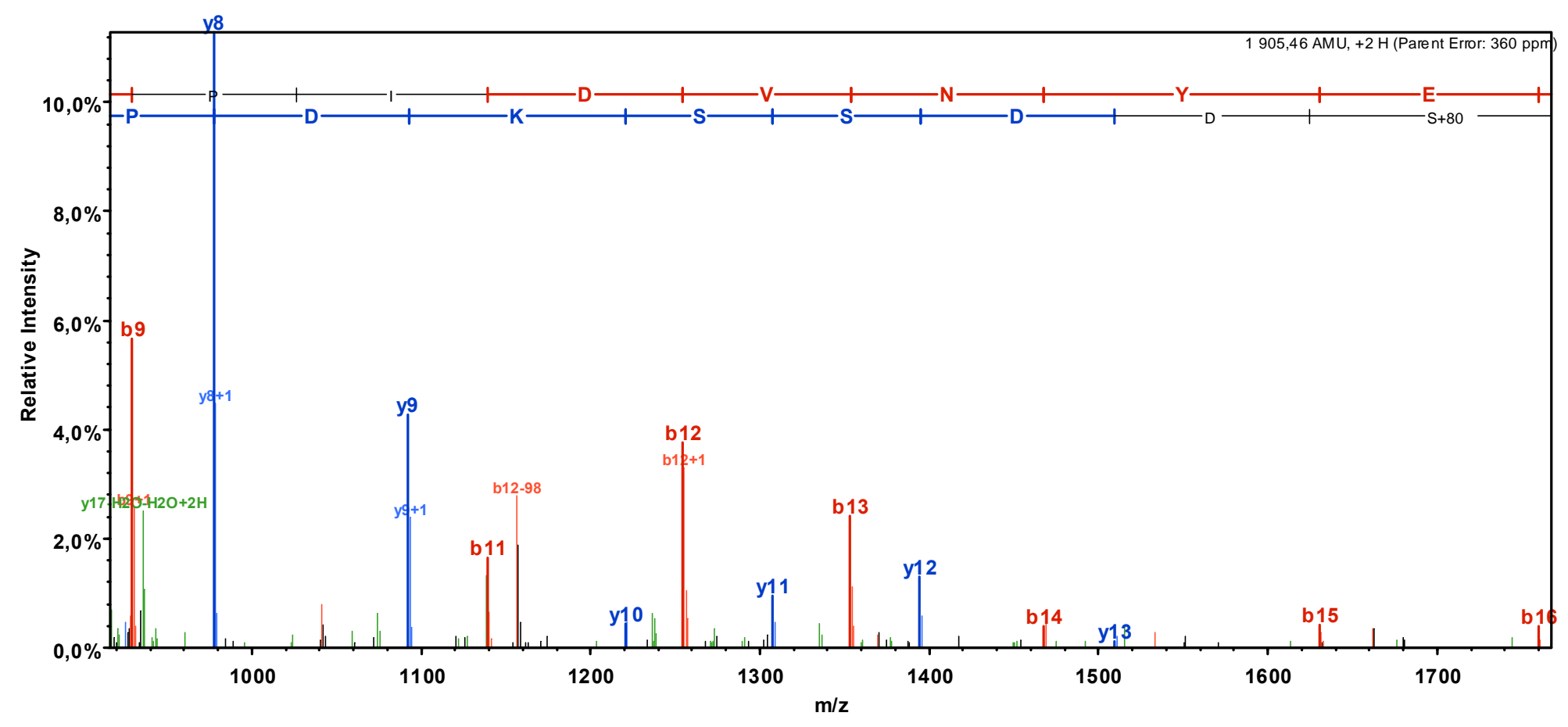


Supplementary mass spectrometric data:

PARP-1,

phosphorylated with Cyclin-dependent kinase-5 (CDK5)

Spectrum Ingel8473.10404.10404.2.dta (charge state: +2)

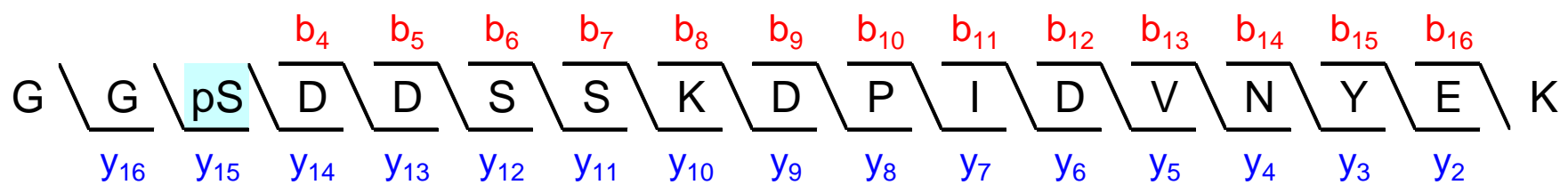

Sequest: XCorr $=4.56, \mathrm{dCn} 1=0.10$ (between $1^{\text {st }}$ and $2^{\text {nd }}$ best matches), $\mathrm{dCn} 2=0.13$ (between $2^{\text {nd }}$ and $3^{\text {rd }}$ best matches), $\mathrm{dCn} 2=$ 0.91 (between $3^{\text {rd }}$ and $4^{\text {th }}$ best matches).

$\mathrm{A}_{\text {score }}=13.49$.

Sequest's second and third best matches with XCorr $=4.12$ and XCorr $=3.96$ respectively are to the same peptide sequence but with alternative phosphorylation sites:

\section{GGSDDPSSKDPIDVNYEK}

GGPSDDSpSKDPIDVNYEK

The very weak Ascore indicates that, given the observed MS/MS spectrum, the phosphopeptide identified could either be Sequest's first, second or third best match (Nat Biotechnol. 2006;24(10):1285-92).

\section{MS/MS spectrum's fragmentation table}

\begin{tabular}{|c|c|c|c|c|c|c|c|c|c|c|}
\hline B & B Ions & $\mathrm{B}+2 \mathrm{H}$ & $\mathrm{B}-\mathrm{NH} 3$ & $\mathrm{~B}-\mathrm{H} 2 \mathrm{O}$ & AA & $Y$ Ions & $\mathrm{Y}+2 \mathrm{H}$ & $\mathrm{Y}-\mathrm{NH} 3$ & $\mathrm{Y}-\mathrm{H} 2 \mathrm{O}$ & $Y$ \\
\hline 1 & 58,0 & & & & G & 1905,8 & 953,4 & 1888,7 & 1887,8 & 17 \\
\hline 2 & 115,1 & & & & G & 1848,8 & 924.9 & 1831,7 & 1830,7 & 16 \\
\hline 3 & 282.0 & & & 264,0 & $S+80$ & 1791,7 & 896.4 & 1774,7 & 1773,7 & 15 \\
\hline 4 & 397.1 & & & 379,1 & D & 1624,7 & 812.9 & 1607,7 & 1606,7 & 14 \\
\hline 5 & 512.1 & & & 494,1 & D & 1509.7 & 755.4 & 1492,7 & 1491,7 & 13 \\
\hline 6 & 599.1 & 300,1 & & 581,1 & S & 1394.7 & 697.8 & 1377.7 & 1376,7 & 12 \\
\hline 7 & 686.2 & 343.6 & & 668.2 & S & 1307.6 & 654,3 & 1290.6 & 1289,6 & 11 \\
\hline 8 & 814.3 & 407.6 & 797,2 & 796.3 & K & 1220.6 & 610,8 & 1203.6 & 1202,6 & 10 \\
\hline 9 & 929.3 & 465.1 & 912,3 & 911.3 & D & 1092.5 & 546,8 & 1075.5 & 1074.5 & 9 \\
\hline 10 & 1026.3 & 513,7 & 1009,3 & 1008,3 & $\mathbf{P}$ & 977.5 & 489.3 & 960.5 & 959.5 & 8 \\
\hline 11 & 1139,4 & 570,2 & 1122.4 & 1121.4 & I & 880.4 & 440.7 & 863.4 & 862,4 & 7 \\
\hline 12 & 1254.5 & 627.7 & 1237.4 & 1236.4 & D & 767.4 & 384,2 & 750.3 & 749.3 & 6 \\
\hline 13 & 1353.5 & 677,3 & 1336.5 & 1335.5 & V & 652,3 & & 635.3 & 634.3 & 5 \\
\hline 14 & 1467.6 & 734.3 & 1450.5 & 1449.6 & $\mathrm{~N}$ & 553.3 & & 536.2 & 535,3 & 4 \\
\hline 15 & 1630.6 & 815,8 & 1613,6 & 1612,6 & $\mathrm{Y}$ & 439.2 & & 422.2 & 421,2 & 3 \\
\hline 16 & 1759.7 & 880,3 & 1742,6 & 1741.7 & E & 276.2 & & 259,1 & 258,1 & 2 \\
\hline 17 & 1905,8 & 953.4 & 1888,7 & 1887,8 & K & 147,1 & & 130,1 & & 1 \\
\hline
\end{tabular}




\section{MS/MS spectrum}

\section{Full-range}

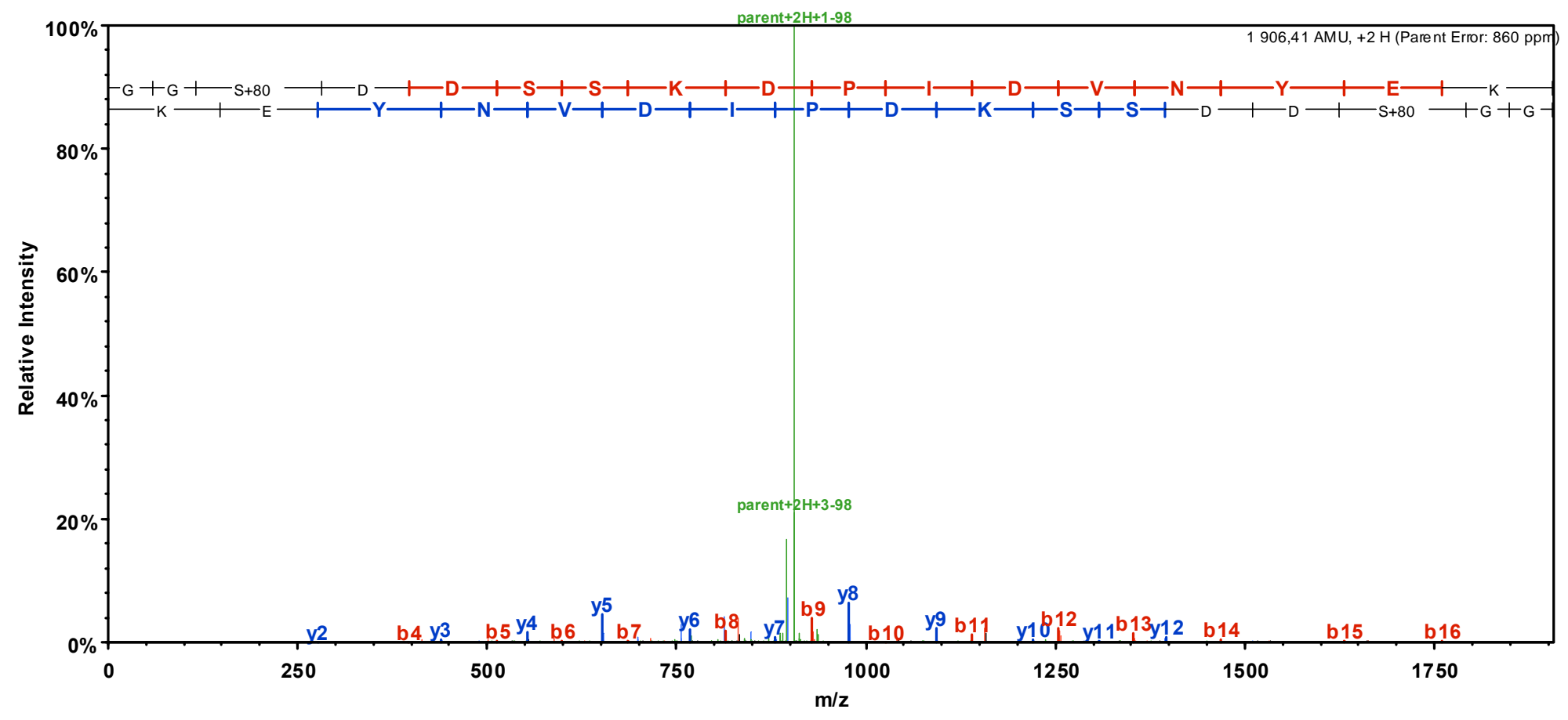

\section{$\underline{\text { Close to precursor }}$}

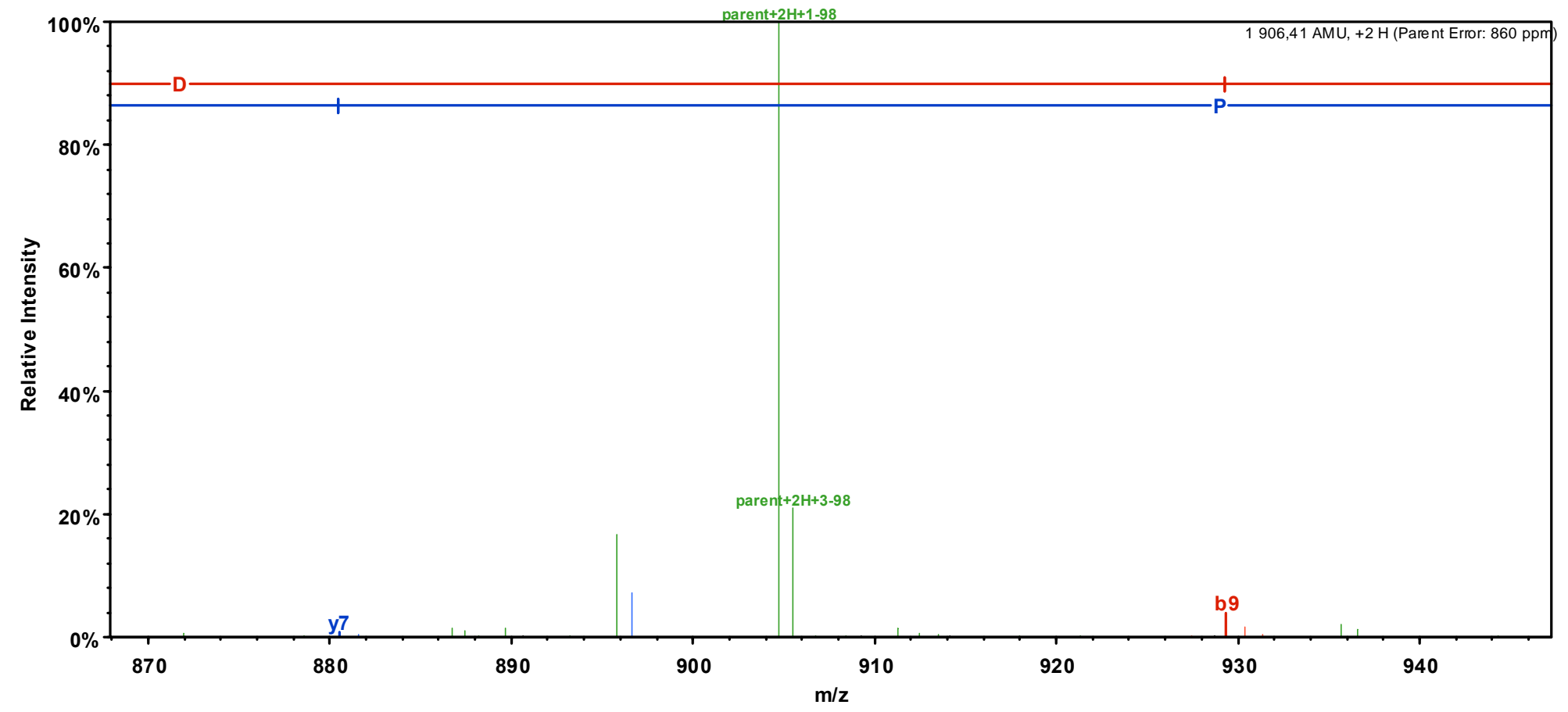




\section{$\underline{\text { Left of precursor }}$}

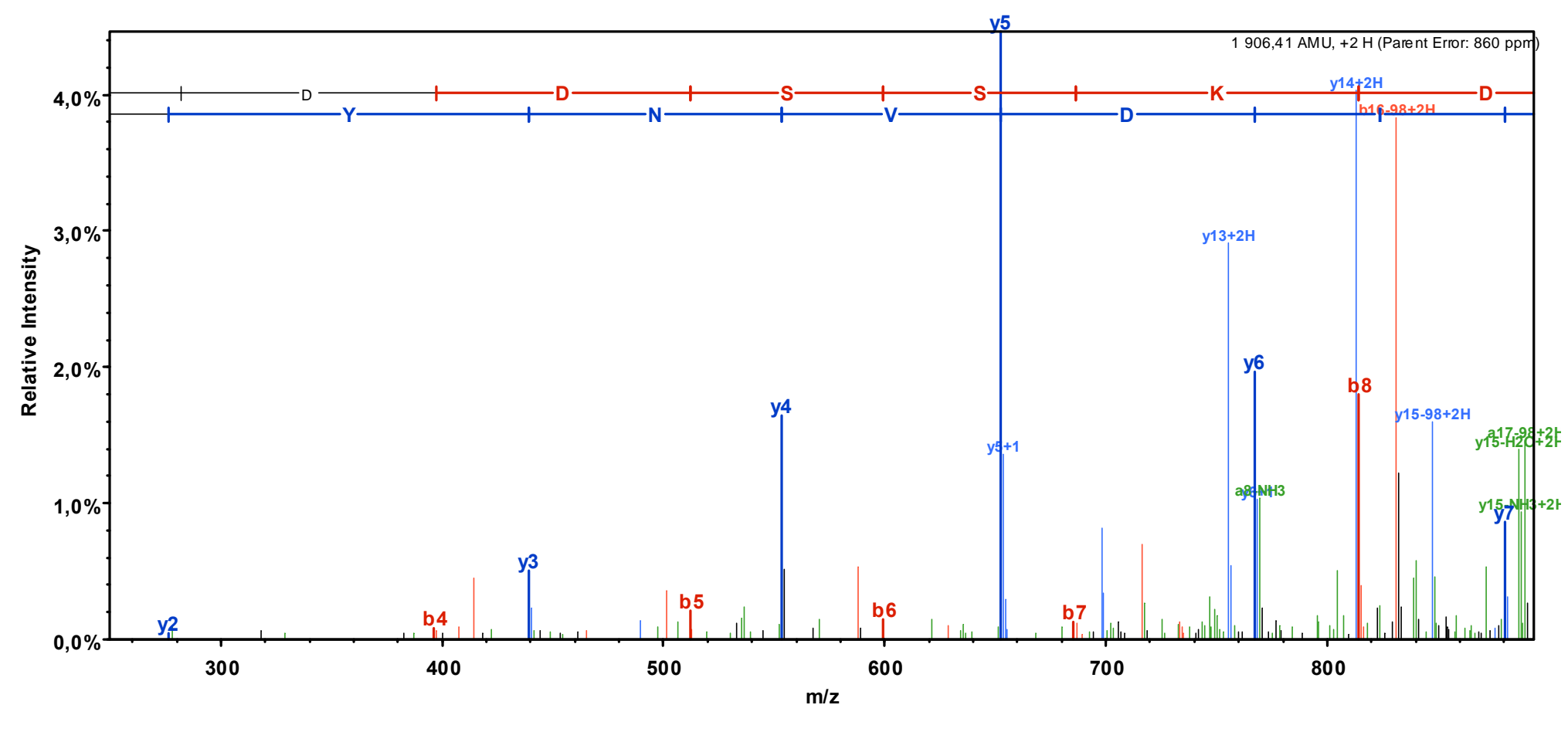

\section{$\underline{\text { Right of precursor }}$}

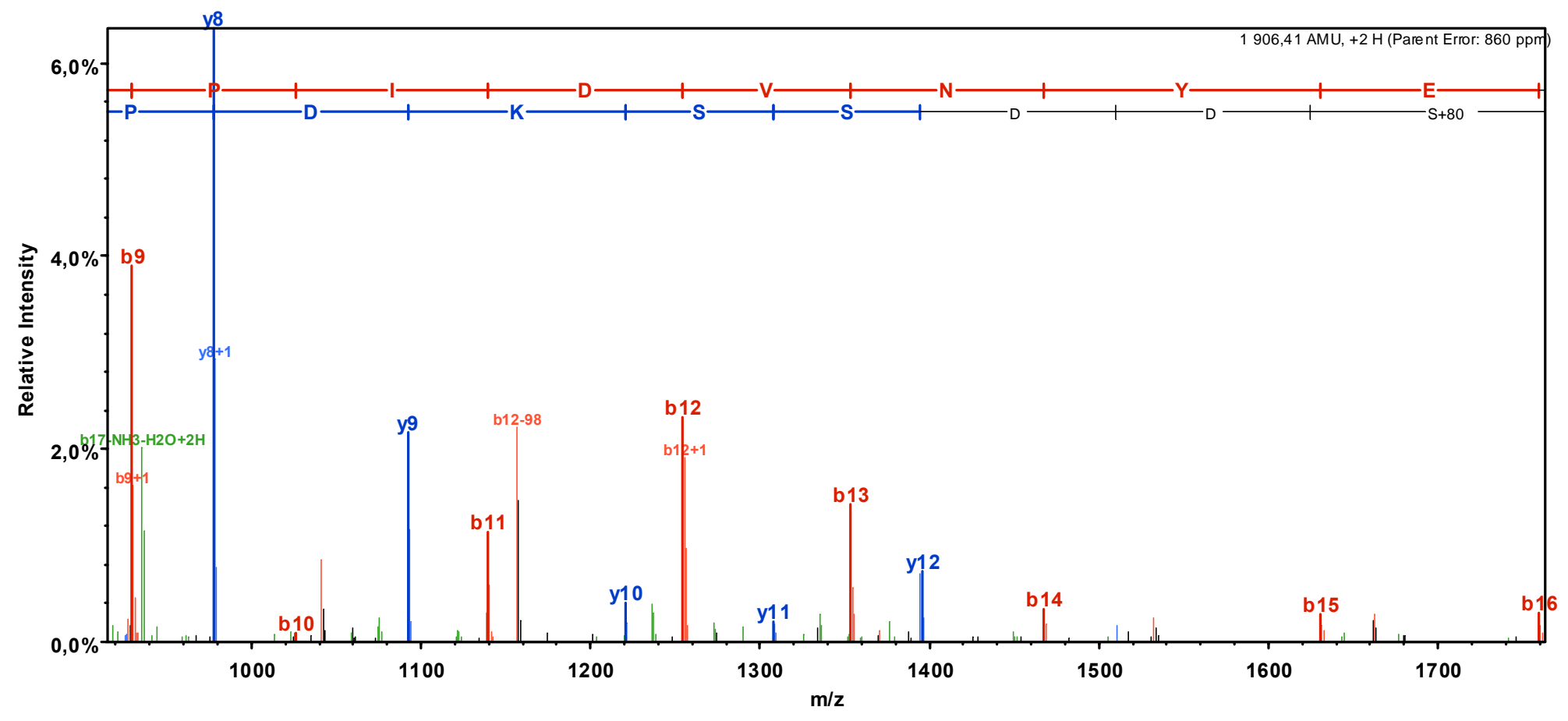


Supplementary mass spectrometric data:

PARG, Casein kinase II-phosphorylated

\section{Spectrum 7806.3163.3163.3.dta (charge state: +3)}

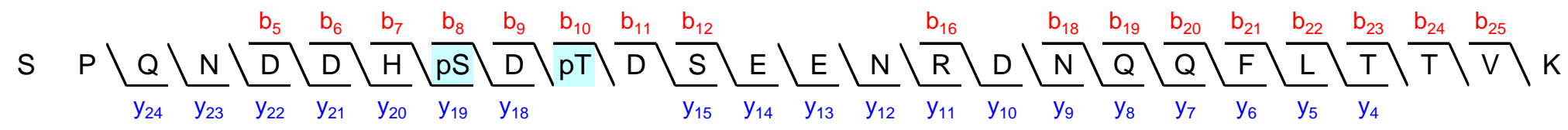

Sequest: XCorr $=4.93, \mathrm{dCn}=0.11$.

Ascore $=23.16$ (first phosphorylation), 29.85 (second phosphorylation).

Both phosphorylation sites are localized with $\geq 99 \%$ certainty as Ascore $\geq 20$ (Nat Biotechnol. 2006;24(10):1285-92).

\section{MS/MS spectrum's fragmentation table}

\begin{tabular}{|c|c|c|c|c|c|c|c|c|c|c|}
\hline B & B Ions & $\mathrm{B}+2 \mathrm{H}$ & $\mathrm{B}-\mathrm{NH} 3$ & $\mathrm{~B}-\mathrm{H} 2 \mathrm{O}$ & AA & $Y$ Ions & $\mathrm{Y}+2 \mathrm{H}$ & $\mathrm{Y}-\mathrm{NH} 3$ & $\mathrm{Y}-\mathrm{H} 2 \mathrm{O}$ & $Y$ \\
\hline 1 & 88,0 & & & 70.0 & $\mathrm{~S}$ & 3180,2 & 1590,6 & 3163,2 & 3162,2 & 26 \\
\hline 2 & 185.1 & & & 167,1 & $\mathbf{P}$ & 3093,2 & 1547,1 & 3076,2 & 3075,2 & 25 \\
\hline 3 & 313,2 & & 296.1 & 295,1 & $\mathbf{Q}$ & 2996.2 & 1498.6 & 2979.1 & 2978,1 & 24 \\
\hline 4 & 427,2 & & 410,2 & 409,2 & $\mathrm{~N}$ & 2868,1 & 1434.6 & 2851,1 & 2850,1 & 23 \\
\hline 5 & 542.2 & & 525,2 & 524,2 & D & 2754,1 & 1377.5 & 2737,0 & 2736.0 & 22 \\
\hline 6 & 657.2 & 329,1 & 640.2 & 639,2 & D & 2639,0 & 1320.0 & 2622.0 & 2621,0 & 21 \\
\hline 7 & 794.3 & 397,7 & 777,3 & 776,3 & $\mathbf{H}$ & 2524,0 & 1262.5 & 2507,0 & 2506,0 & 20 \\
\hline 8 & 961.3 & 481,2 & 944.3 & 943.3 & $S+80$ & 2386,9 & 1194.0 & 2369,9 & 2368.9 & 19 \\
\hline 9 & 1076.3 & 538,7 & 1059,3 & 1058,3 & D & 2219,9 & 1110.5 & 2202.9 & 2201,9 & 18 \\
\hline 10 & 1257.3 & 629,2 & 1240.3 & 1239.3 & $\mathbf{T}+\mathbf{8 0}$ & 2104,9 & 1053,0 & 2087,9 & 2086,9 & 17 \\
\hline 11 & 1372.4 & 686,7 & 1355,3 & 1354,4 & D & 1923,9 & 962.5 & 1906.9 & 1905,9 & 16 \\
\hline 12 & 1459.4 & 730,2 & 1442,4 & 1441,4 & S & 1808,9 & 904.9 & 1791,9 & 1790,9 & 15 \\
\hline 13 & 1588.4 & 794,7 & 1571,4 & 1570,4 & $\mathrm{E}$ & 1721,8 & 861.4 & 1704.8 & 1703.8 & 14 \\
\hline 14 & 1717.5 & 859,2 & 1700.5 & 1699.5 & $E$ & 1592,8 & 796.9 & 1575,8 & 1574,8 & 13 \\
\hline 15 & 1831,5 & 916.3 & 1814,5 & 1813.5 & $\mathrm{~N}$ & 1463,8 & 732.4 & 1446.7 & 1445,8 & 12 \\
\hline 16 & 1987,6 & \begin{tabular}{|l|l|}
994.3 \\
\end{tabular} & 1970,6 & 1969,6 & $\mathbf{R}$ & 1349.7 & 675.4 & 1332,7 & 1331,7 & 11 \\
\hline 17 & 2102,7 & 1051,8 & 2085,6 & 2084,7 & D & 1193.6 & 597,3 & 1176.6 & 1175,6 & 10 \\
\hline 18 & 2216.7 & 1108.9 & 2199,7 & 2198,7 & $\mathrm{~N}$ & 1078.6 & 539,8 & 1061,6 & 1060,6 & 9 \\
\hline 19 & 2344,8 & 1172.9 & 2327,7 & 2326,8 & $\mathbf{Q}$ & 964,5 & 482,8 & 947.5 & 946,5 & 8 \\
\hline 20 & 2472,8 & 1236.9 & 2455,8 & 2454,8 & $\mathbf{Q}$ & 836.5 & 418,7 & 819.5 & 818.5 & 7 \\
\hline 21 & 2619,9 & 1310.4 & 2602,9 & 2601,9 & $F$ & 708.4 & 354,7 & 691,4 & 690,4 & 6 \\
\hline 22 & 2733,0 & 1367.0 & 2715,9 & 2715,0 & L & 561.4 & & 544.3 & 543,4 & 5 \\
\hline 23 & 2834,0 & 1417.5 & 2817,0 & 2816,0 & $\mathbf{T}$ & 448.3 & & 431,3 & 430,3 & 4 \\
\hline 24 & 2935,1 & 1468.0 & 2918,0 & 2917,1 & $\mathbf{T}$ & 347,2 & & 330,2 & 329,2 & 3 \\
\hline 25 & 3034.1 & 1517.6 & 3017,1 & 3016,1 & V & 246,2 & & 229,2 & & 2 \\
\hline 26 & 3180,2 & 1590,6 & 3163.2 & 3162,2 & K & 147,1 & & 130,1 & & 1 \\
\hline
\end{tabular}




\section{MS/MS spectrum}

\section{Full-range}

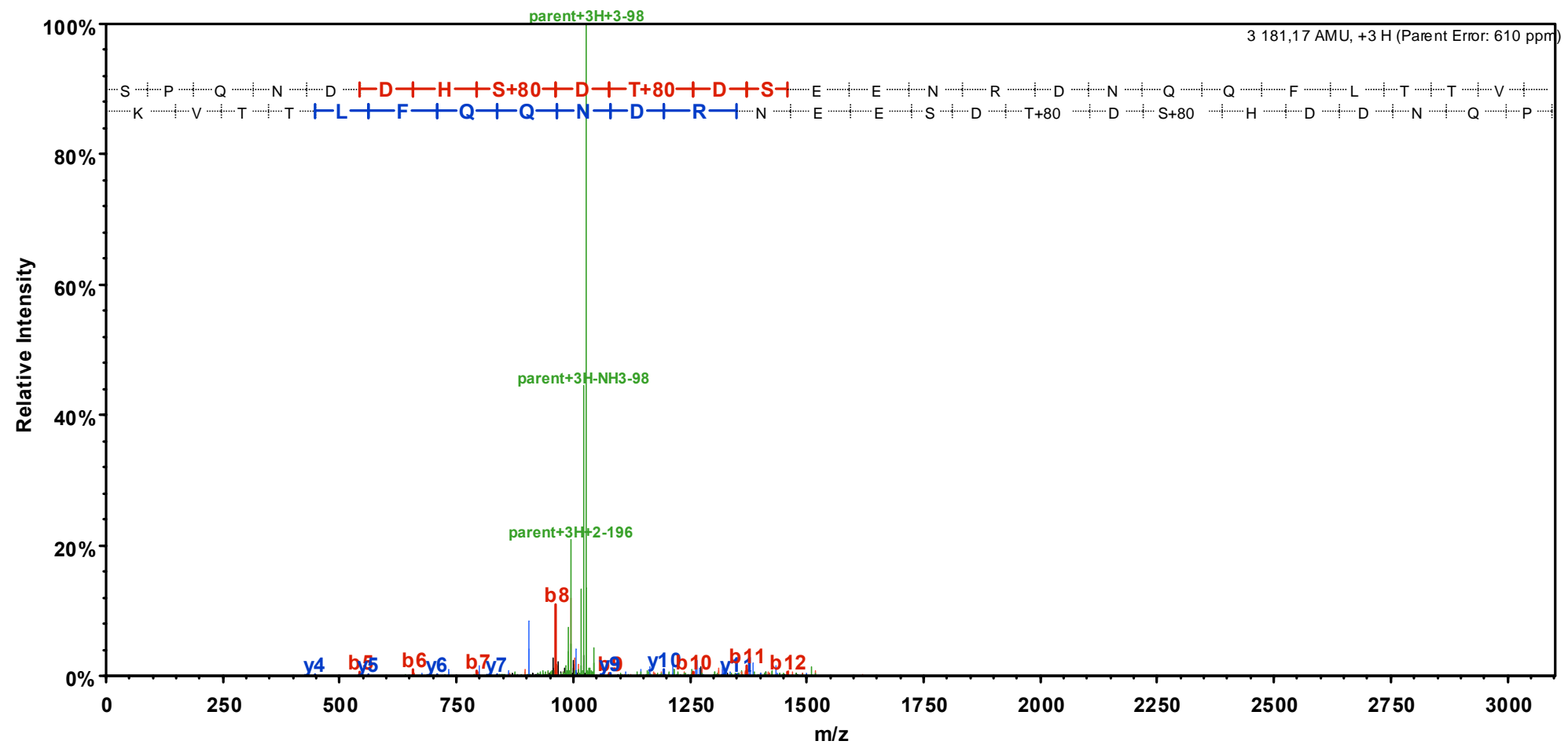

\section{Close to precursor}

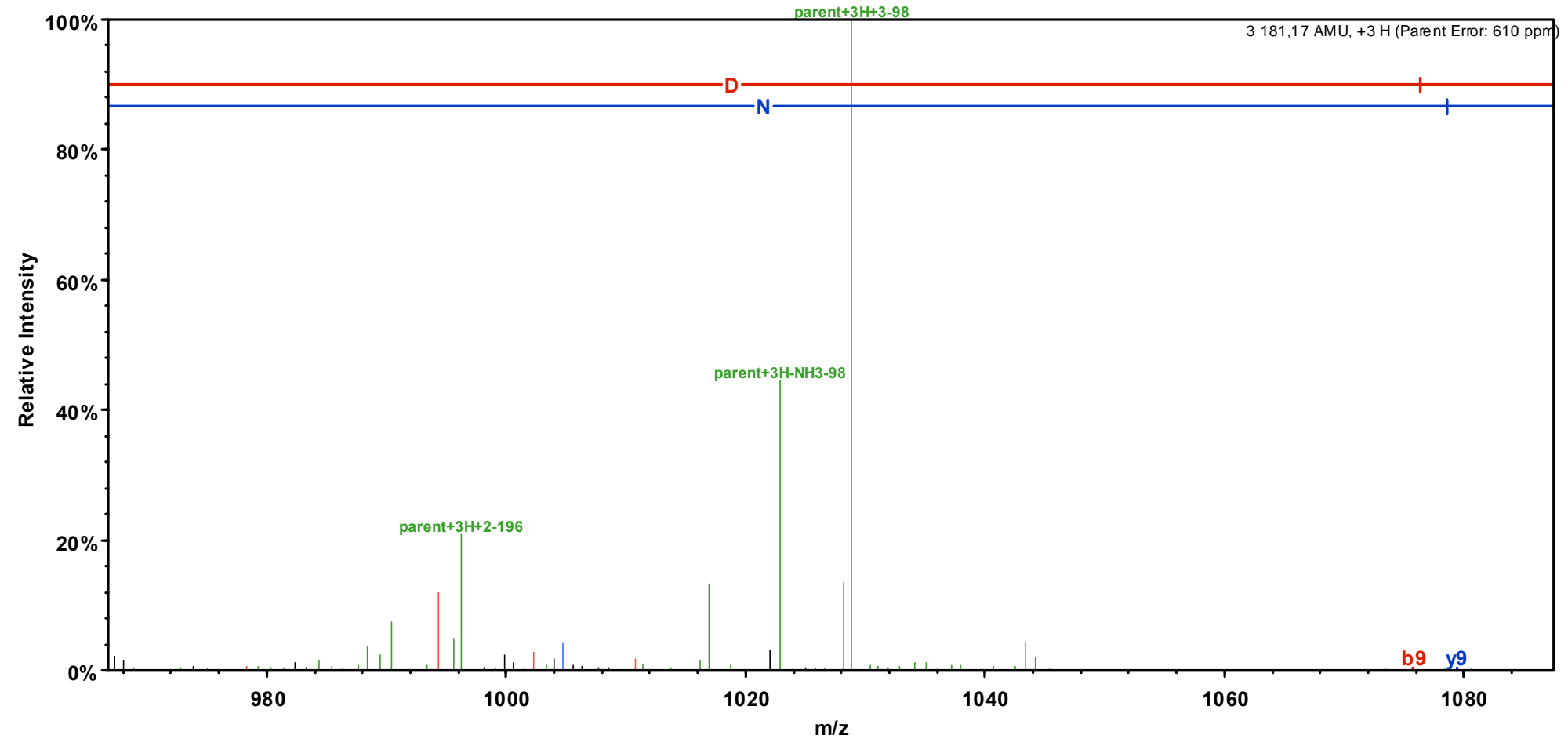




\section{$\underline{\text { Left of precursor }}$}

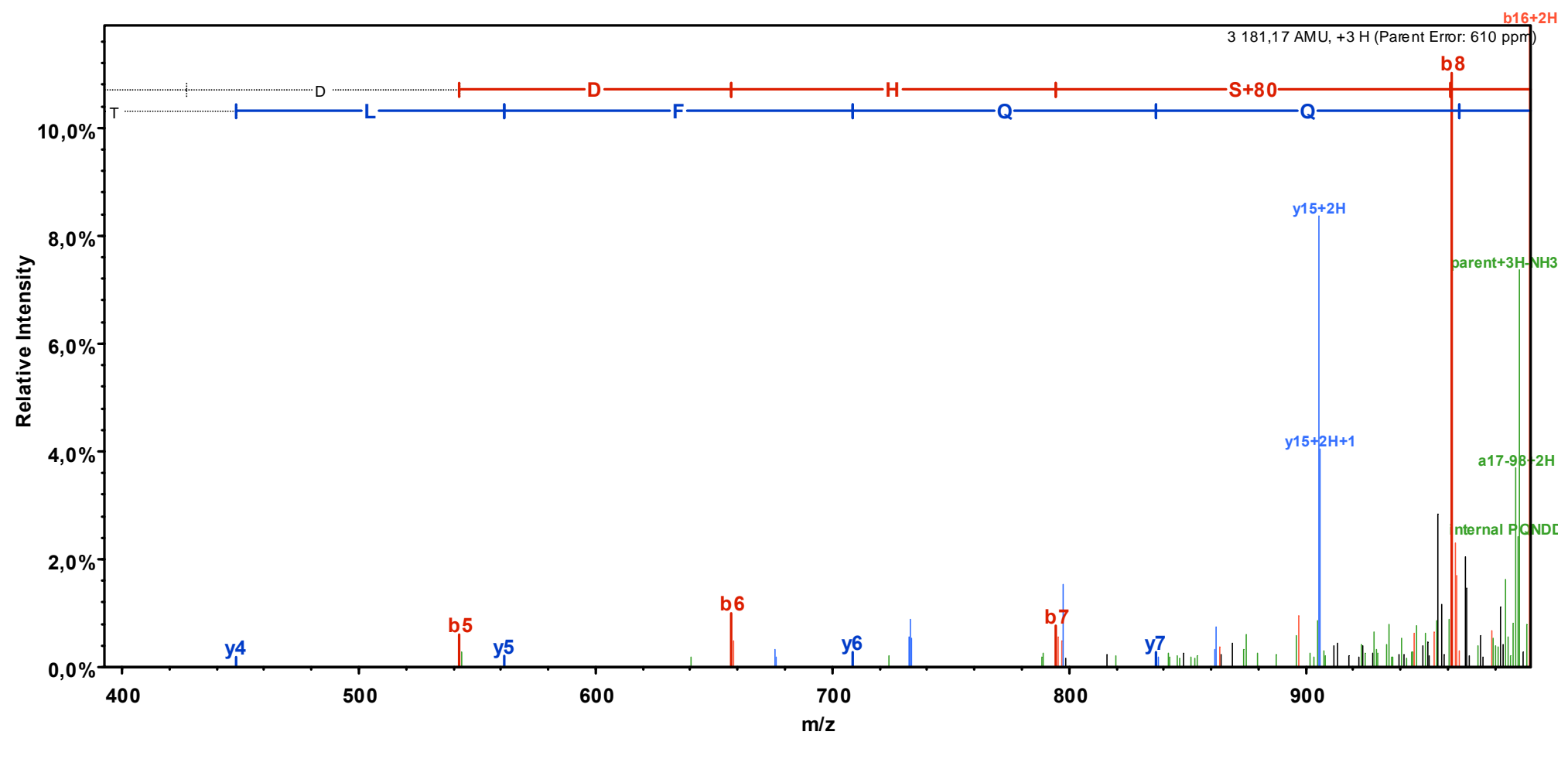

\section{$\underline{\text { Right of precursor }}$}

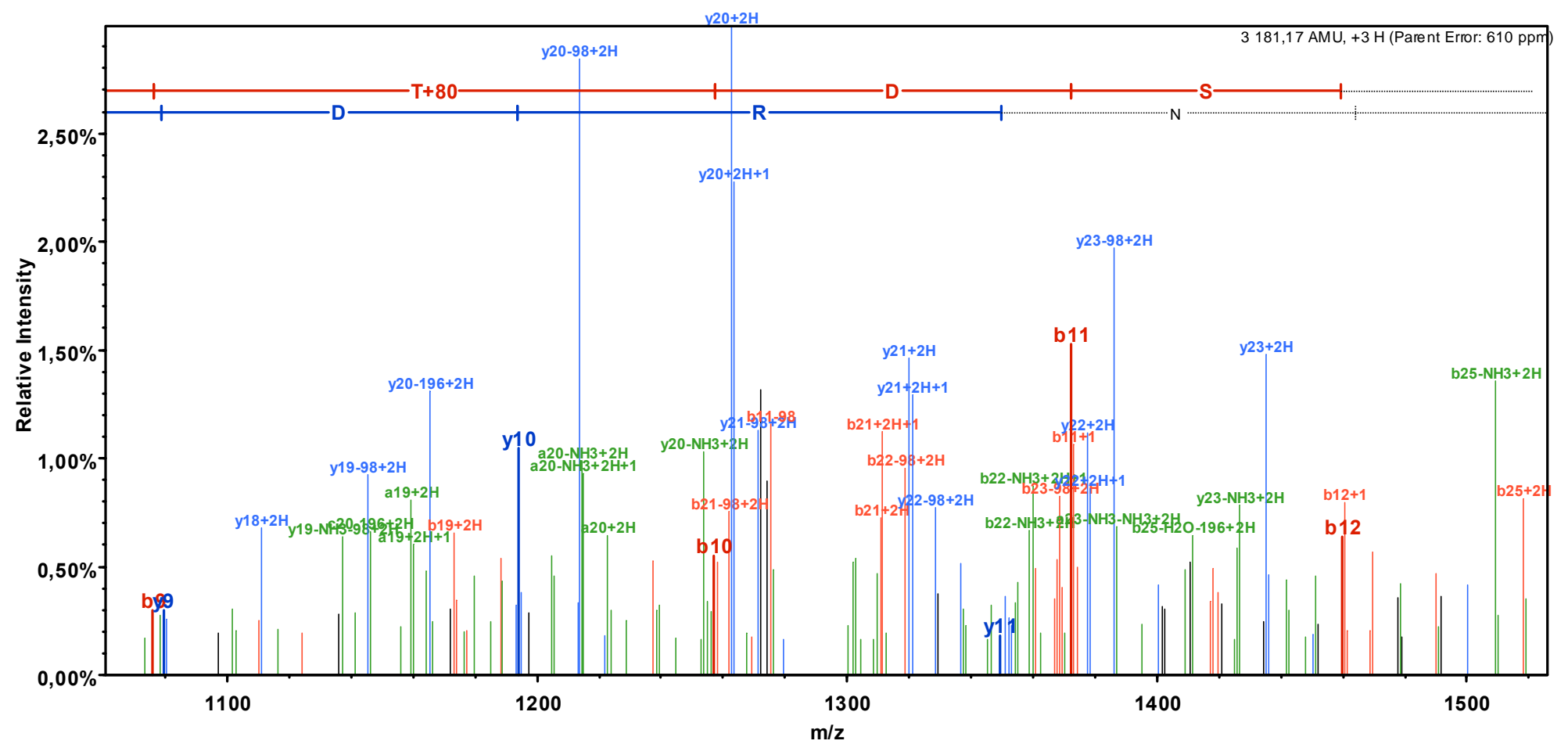




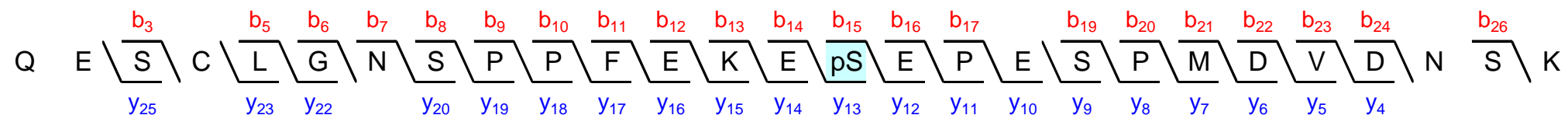

Sequest: XCorr $=4.97, \mathrm{dCn}=0.10$.

Ascore $=26.34$.

Phosphorylation site is localized with $\geq 99 \%$ certainty as Ascore $\geq 20$ (Nat Biotechnol. 2006;24(10):1285-92).

\section{MS/MS spectrum's fragmentation table}

\begin{tabular}{|c|c|c|c|c|c|c|c|c|c|c|}
\hline B & B Ions & $\mathrm{B}+2 \mathrm{H}$ & $\mathrm{B}-\mathrm{NH} 3$ & $\mathrm{~B}-\mathrm{H} 2 \mathrm{O}$ & AA & $Y$ Ions & $\mathrm{Y}+2 \mathrm{H}$ & $\mathrm{Y}-\mathrm{NH} 3$ & $\mathrm{Y}-\mathrm{H} 2 \mathrm{O}$ & $Y$ \\
\hline 1 & 129,1 & & 112,0 & & $\mathbf{Q}$ & 3133,3 & 1567.1 & 3116,2 & 3115,3 & 27 \\
\hline 2 & 258,1 & & 241,1 & 240,1 & $E$ & 3005,2 & 1503.1 & 2988,2 & 2987,2 & 26 \\
\hline 3 & 345,1 & & 328,1 & 327.1 & $\mathrm{~S}$ & 2876,2 & 1438.6 & 2859,1 & 2858,2 & 25 \\
\hline 4 & 505,2 & & 488,1 & 487,2 & $C+57$ & 2789,1 & 1395,1 & 2772,1 & 2771,1 & 24 \\
\hline 5 & 618.3 & & 601,2 & 600.2 & L & 2629,1 & 1315.1 & 2612,1 & 2611,1 & 23 \\
\hline 6 & 675.3 & 338,1 & 658.3 & 657,3 & G & 2516,0 & 1258.5 & 2499,0 & 2498,0 & 22 \\
\hline 7 & 789.3 & 395,2 & 772.3 & 771,3 & $\mathrm{~N}$ & 2459,0 & 1230,0 & 2442,0 & 2441,0 & 21 \\
\hline 8 & 876.4 & 438.7 & 859.3 & 858.3 & S & 2345,0 & 1173.0 & 2327,9 & 2326,9 & 20 \\
\hline 9 & 973.4 & 487,2 & 956.4 & 955,4 & $\mathbf{P}$ & 2257,9 & 1129.5 & 2240,9 & 2239,9 & 19 \\
\hline 10 & 1070.5 & 535,7 & 1053.4 & 1052,4 & $\mathbf{P}$ & 2160,9 & 1080.9 & 2143,8 & 2142,9 & 18 \\
\hline 11 & 1217.5 & 609.3 & 1200.5 & 1199,5 & $F$ & 2063,8 & 1032.4 & 2046,8 & 2045,8 & 17 \\
\hline 12 & 1346.6 & 673.8 & 1329.5 & 1328.6 & $E$ & 1916.7 & 958.9 & 1899,7 & 1898.7 & 16 \\
\hline 13 & 1474.7 & 737.8 & 1457.6 & 1456.7 & K & 1787.7 & 894.4 & 1770,7 & 1769,7 & 15 \\
\hline 14 & 1603,7 & 802,4 & 1586.7 & 1585,7 & $E$ & 1659.6 & 830.3 & 1642.6 & 1641,6 & 14 \\
\hline 15 & 1770,7 & 885.9 & 1753,7 & 1752,7 & $S+80$ & 1530.6 & 765,8 & 1513,5 & 1512,6 & 13 \\
\hline 16 & 1899,7 & 950.4 & 1882.7 & 1881,7 & E & 1363.6 & 682,3 & 1346.5 & 1345,6 & 12 \\
\hline 17 & 1996,8 & 998.9 & 1979,8 & 1978.8 & $\mathbf{P}$ & 1234.5 & 617,8 & 1217.5 & 1216.5 & 11 \\
\hline 18 & 2125,8 & 1063.4 & 2108,8 & 2107,8 & $E$ & 1137.5 & 569,2 & 1120.4 & 1119.5 & 10 \\
\hline 19 & 2212,9 & 1106.9 & 2195,8 & 2194,9 & S & 1008.4 & 504,7 & 991,4 & 990.4 & 9 \\
\hline 20 & 2309,9 & 1155.5 & 2292,9 & 2291,9 & $\mathbf{P}$ & 921.4 & 461.2 & 904.4 & 903.4 & 8 \\
\hline 21 & 2457,0 & 1229.0 & 2439,9 & 2439.0 & $M+16$ & 824.3 & 412,7 & 807,3 & 806,3 & 7 \\
\hline 22 & 2572,0 & 1286.5 & 2555,0 & 2554,0 & D & 677.3 & 339,2 & 660,3 & 659,3 & 6 \\
\hline 23 & 2671,1 & 1336.0 & 2654.0 & 2653,0 & V & 562.3 & & 545,3 & 544,3 & 5 \\
\hline 24 & 2786.1 & 1393.5 & 2769,1 & 2768.1 & D & 463.2 & & 446.2 & 445,2 & 4 \\
\hline 25 & 2900,1 & 1450,6 & 2883,1 & 2882,1 & $\mathrm{~N}$ & 348,2 & & 331,2 & 330,2 & 3 \\
\hline 26 & 2987,2 & 1494.1 & 2970,1 & 2969,1 & S & 234,1 & & 217,1 & 216,1 & 2 \\
\hline 27 & 3133,3 & 1567,1 & 3116,2 & 3115,3 & K & 147,1 & & 130,1 & & 1 \\
\hline
\end{tabular}




\section{MS/MS spectrum}

Full-range

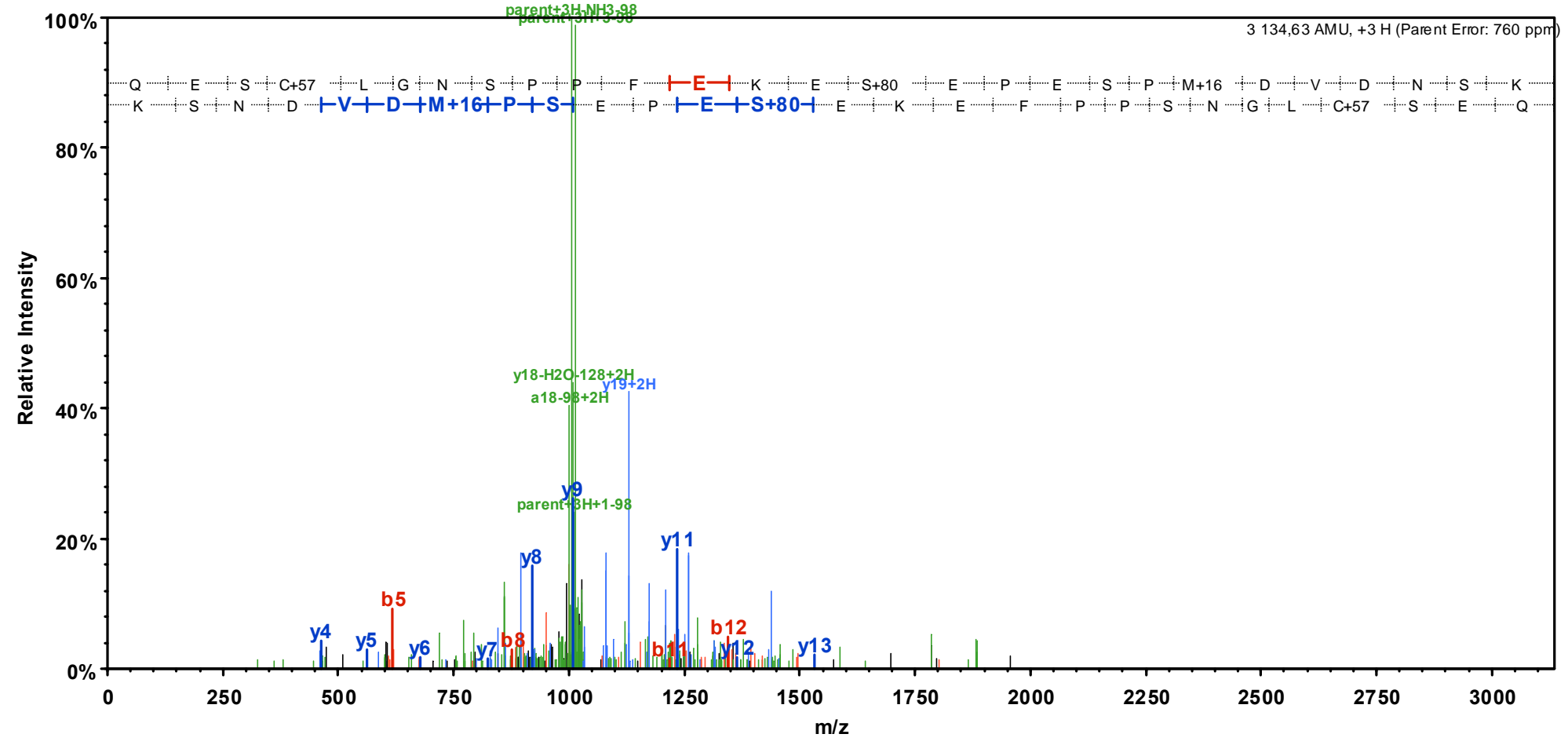

\section{Close to precursor}

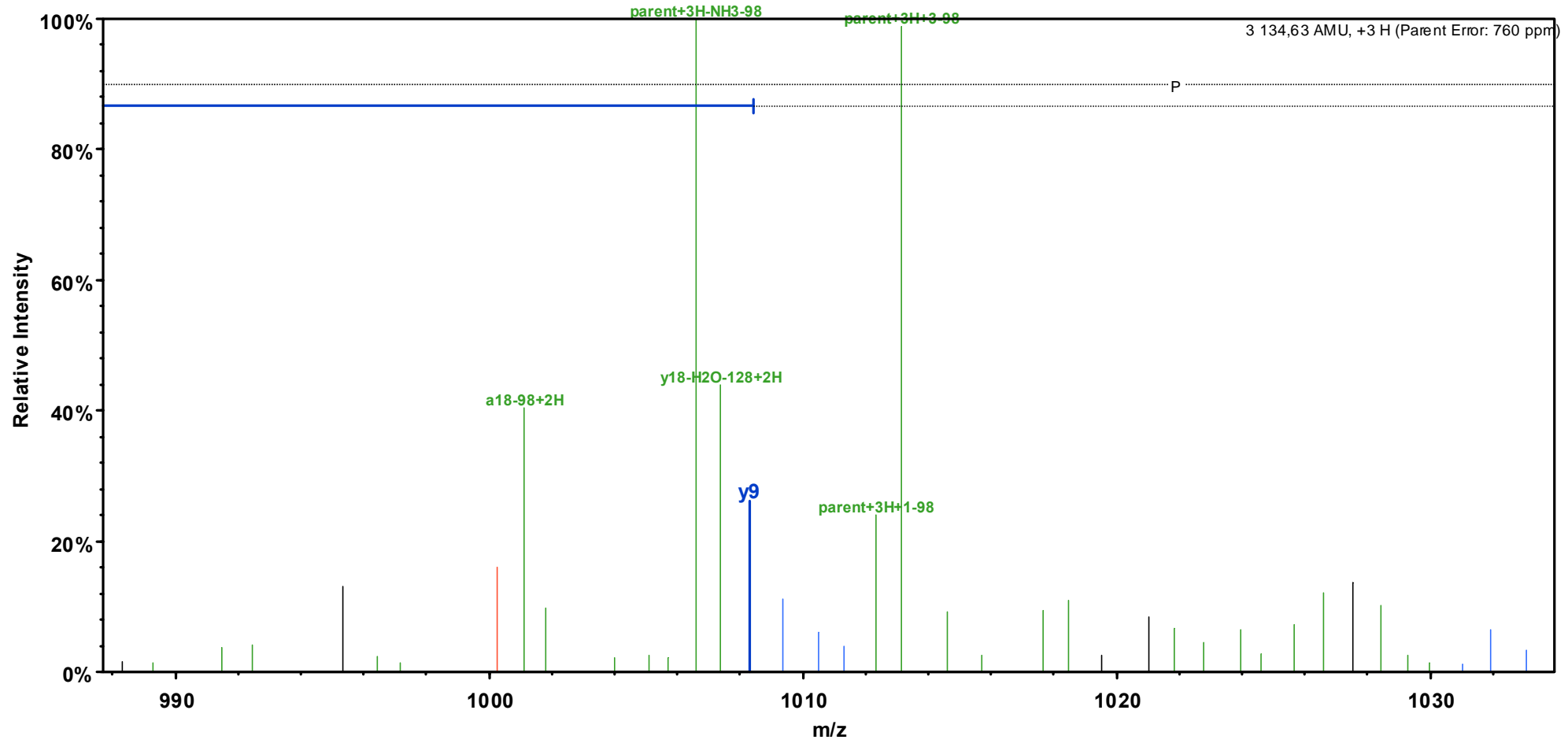




\section{Left of precursor}

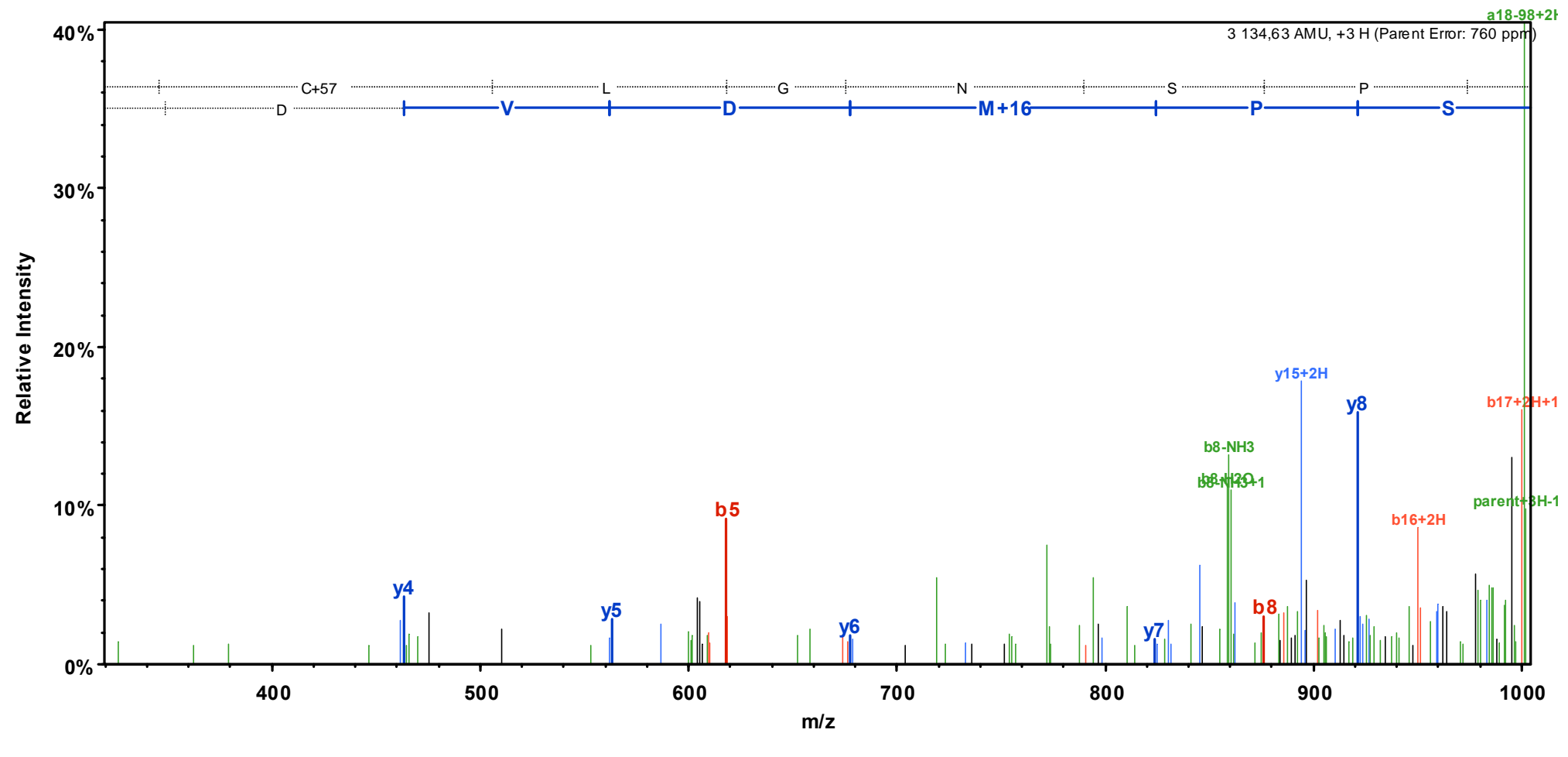

\section{$\underline{\text { Right of precursor }}$}

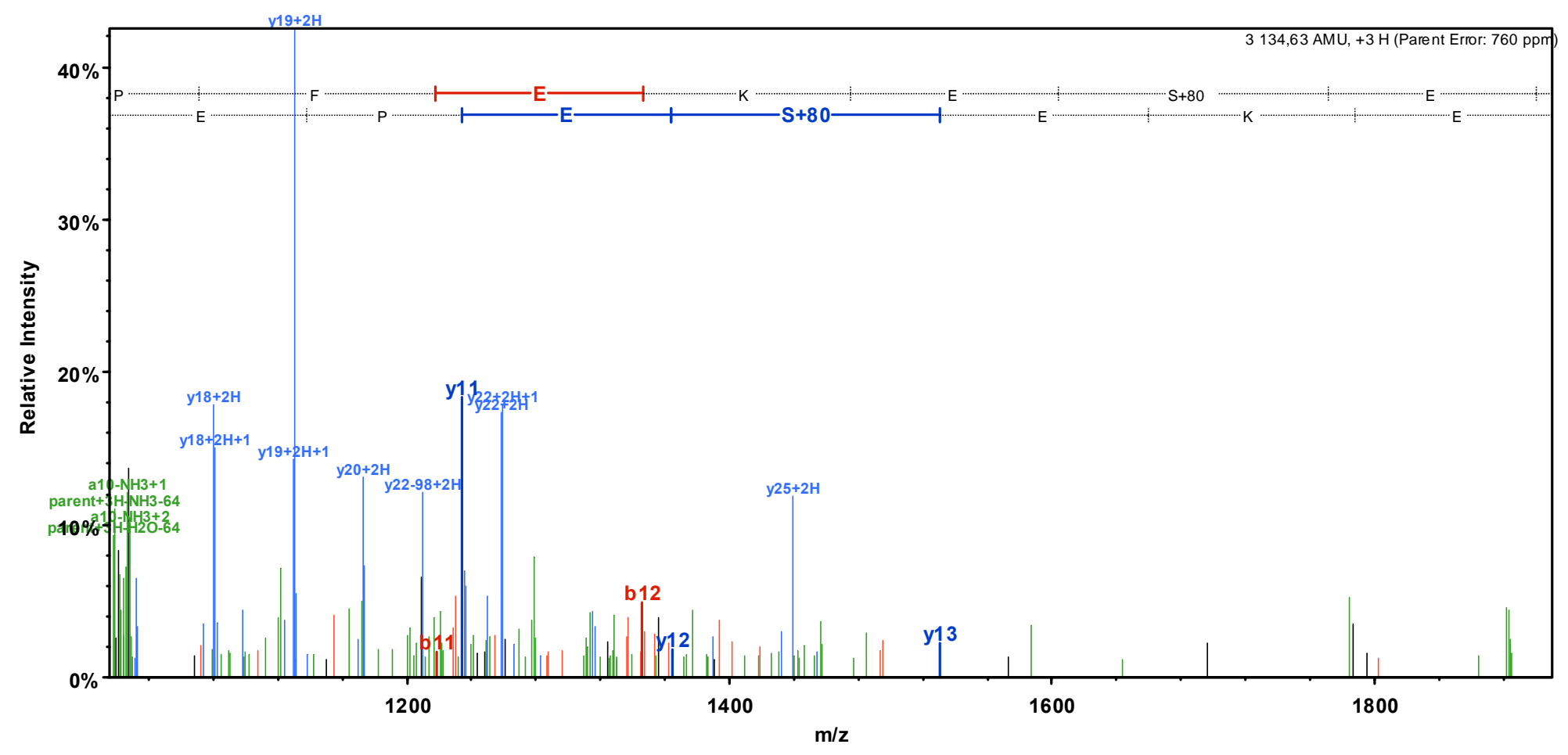




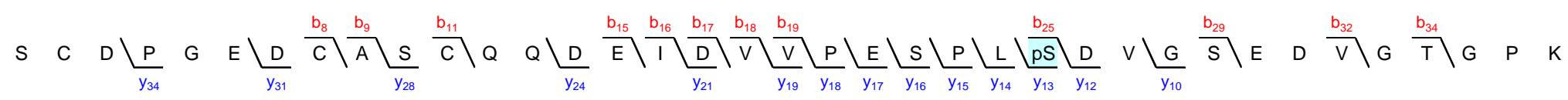

Sequest: $X$ Corr $=2.72, \mathrm{dCn} 1=0.03$ (between $1^{\text {st }}$ and $2^{\text {nd }}$ best matches), $\mathrm{dCn} 2=0.11$ (between $2^{\text {nd }}$ and $3^{\text {rd }}$ best matches). $A_{\text {score }}=0$.

Sequest's second best match with XCorr $=2.63$ is to the same peptide sequence but with an alternative phosphorylation site: SCDPGEDCASCQQDEIDVVPEPSPLSDVGSEDVGTGPK.

The very weak Ascore indicates that, given the observed MS/MS spectrum, the phosphopeptide identified could either be Sequest's first or second best match (Nat Biotechnol. 2006;24(10):1285-92).

\section{MS/MS spectrum's fragmentation table}

\begin{tabular}{|c|c|c|c|c|c|c|c|c|c|c|}
\hline B & B Ions & $\mathrm{B}+2 \mathrm{H}$ & $\mathrm{B}-\mathrm{NH} 3$ & $\mathrm{~B}-\mathrm{H} 2 \mathrm{O}$ & AA & $Y$ Ions & $\mathrm{Y}+2 \mathrm{H}$ & $\mathrm{Y}-\mathrm{NH} 3$ & $\mathrm{Y}-\mathrm{H} 2 \mathrm{O}$ & $\mathrm{Y}$ \\
\hline 1 & $\mathbf{8 8 , 0}$ & & & 70.0 & 5 & 4015,6 & 2008.3 & 3998.6 & 3997,6 & 37 \\
\hline 2 & 248,1 & & & 230,1 & $C+57$ & 3928,6 & 1964,8 & 3911,5 & 3910,6 & 36 \\
\hline 3 & 363,1 & & & 345,1 & D & 3768.5 & 1884,8 & 3751,5 & 3750.5 & 35 \\
\hline 4 & 460,2 & & & 442,1 & $\mathbf{P}$ & 3653.5 & 1827.3 & 3636,5 & 3635.5 & 34 \\
\hline 5 & 517,2 & & & 499,2 & G & 3556.5 & 1778,7 & 3539,4 & 3538,5 & 33 \\
\hline 6 & 646,2 & 323.6 & & 628,2 & $E$ & 3499,4 & 1750,2 & 3482,4 & 3481,4 & 32 \\
\hline 7 & 761,2 & 381,1 & & 743,2 & D & 3370,4 & 1685.7 & 3353.4 & 3352,4 & 31 \\
\hline 8 & 921.3 & 461,1 & & 903,3 & $C+57$ & 3255,4 & 1628,2 & 3238,3 & 3237,4 & 30 \\
\hline 9 & 992,3 & 496.7 & & 974,3 & A & 3095.3 & 1548,2 & 3078.3 & 3077,3 & 29 \\
\hline 10 & 1079,3 & 540,2 & & 1061,3 & $S$ & 3024,3 & 1512.7 & 3007,3 & 3006,3 & 28 \\
\hline 11 & 1239,4 & 620,2 & & 1221.4 & $C+57$ & 2937,3 & 1469,1 & 2920,2 & 2919,3 & 27 \\
\hline 12 & 1367,4 & 684,2 & 1350,4 & 1349,4 & Q & 2777,2 & 1389.1 & 2760,2 & 2759,2 & 26 \\
\hline 13 & 1495,5 & 748,2 & 1478.5 & 1477,5 & $\mathbf{Q}$ & 2649,2 & 1325,1 & 2632,2 & 2631,2 & 25 \\
\hline 14 & 1610,5 & $\mathbf{8 0 5 , 8}$ & 1593.5 & 1592,5 & D & 2521,1 & 1261.1 & 2504,1 & 2503.1 & 24 \\
\hline 15 & 1739.6 & 870.3 & 1722,5 & 1721,5 & $E$ & 2406,1 & 1203,6 & 2389,1 & 2388,1 & 23 \\
\hline 16 & 1852.6 & 926.8 & 1835,6 & 1834.6 & I & 2277.1 & 1139,0 & 2260,0 & 2259,0 & 22 \\
\hline 17 & 1967,7 & 984.3 & 1950,6 & 1949,7 & D & 2164,0 & 1082.5 & 2146.9 & 2146,0 & 21 \\
\hline 18 & 2066.7 & 1033.9 & 2049,7 & 2048,7 & V & 2048.9 & 1025,0 & 2031,9 & 2030,9 & 20 \\
\hline 19 & 2165,8 & 1083.4 & 2148,8 & 2147,8 & V & 1949.9 & 975.4 & 1932,8 & 1931,9 & 19 \\
\hline 20 & 2262,9 & 1131,9 & 2245,8 & 2244,8 & $\mathbf{P}$ & 1850,8 & 925,9 & 1833.8 & 1832,8 & 18 \\
\hline 21 & 2391,9 & 1196.5 & 2374,9 & 2373.9 & $\mathrm{E}$ & 1753.8 & 877.4 & 1736.7 & 1735.7 & 17 \\
\hline 22 & 2478,9 & 1240,0 & 2461,9 & 2460,9 & S & 1624.7 & 812.9 & 1607,7 & 1606.7 & 16 \\
\hline 23 & 2576,0 & 1288,5 & 2559,0 & 2558,0 & $\mathbf{P}$ & 1537.7 & 769,3 & 1520.7 & 1519,7 & 15 \\
\hline 24 & 2689,1 & 1345,0 & 2672,0 & 2671,1 & L & 1440.6 & 720,8 & 1423,6 & 1422,6 & 14 \\
\hline 25 & 2856,1 & 1428.5 & 2839,0 & 2838,1 & $S+80$ & 1327,5 & 664,3 & 1310.5 & 1309.5 & 13 \\
\hline 26 & 2971,1 & 1486,1 & 2954,1 & 2953,1 & D & 1160.5 & 580,8 & 1143,5 & 1142,5 & 12 \\
\hline 27 & 3070,2 & 1535,6 & 3053.1 & 3052,2 & V & 1045.5 & 523,3 & 1028.5 & 1027.5 & 11 \\
\hline 28 & 3127,2 & 1564,1 & 3110,2 & 3109,2 & G & 946.4 & 473,7 & 929.4 & 928.4 & 10 \\
\hline 29 & 3214,2 & 1607.6 & 3197,2 & 3196,2 & S & 889,4 & 445,2 & 872,4 & 871,4 & 9 \\
\hline 30 & 3343,3 & 1672,1 & 3326,2 & 3325,2 & E & 802,4 & 401,7 & 785,4 & 784,4 & 8 \\
\hline 31 & 3458,3 & 1729,6 & 3441,3 & 3440,3 & D & 673,4 & 337,2 & 656,3 & 655,3 & 7 \\
\hline 32 & 3557,4 & 1779.2 & 3540,3 & 3539,3 & V & 558,3 & 279,7 & 541,3 & 540,3 & 6 \\
\hline 33 & 3614,4 & 1807.7 & 3597,4 & 3596,4 & G & 459,3 & & 442,2 & 441,2 & 5 \\
\hline 34 & 3715,4 & 1858.2 & 3698,4 & 3697,4 & $\mathbf{T}$ & 402,2 & & 385,2 & 384,2 & 4 \\
\hline 35 & 3772,4 & 1886,7 & 3755,4 & 3754,4 & G & 301,2 & & 284,2 & & 3 \\
\hline 36 & 3869.5 & 1935.3 & 3852.5 & 3851.5 & $\mathbf{P}$ & 244,2 & & 227,1 & & 2 \\
\hline 37 & 4015,6 & 2008.3 & 3998.6 & 3997.6 & $K$ & 147,1 & & 130,1 & & 1 \\
\hline
\end{tabular}




\section{MS/MS spectrum}

\section{Full-range}

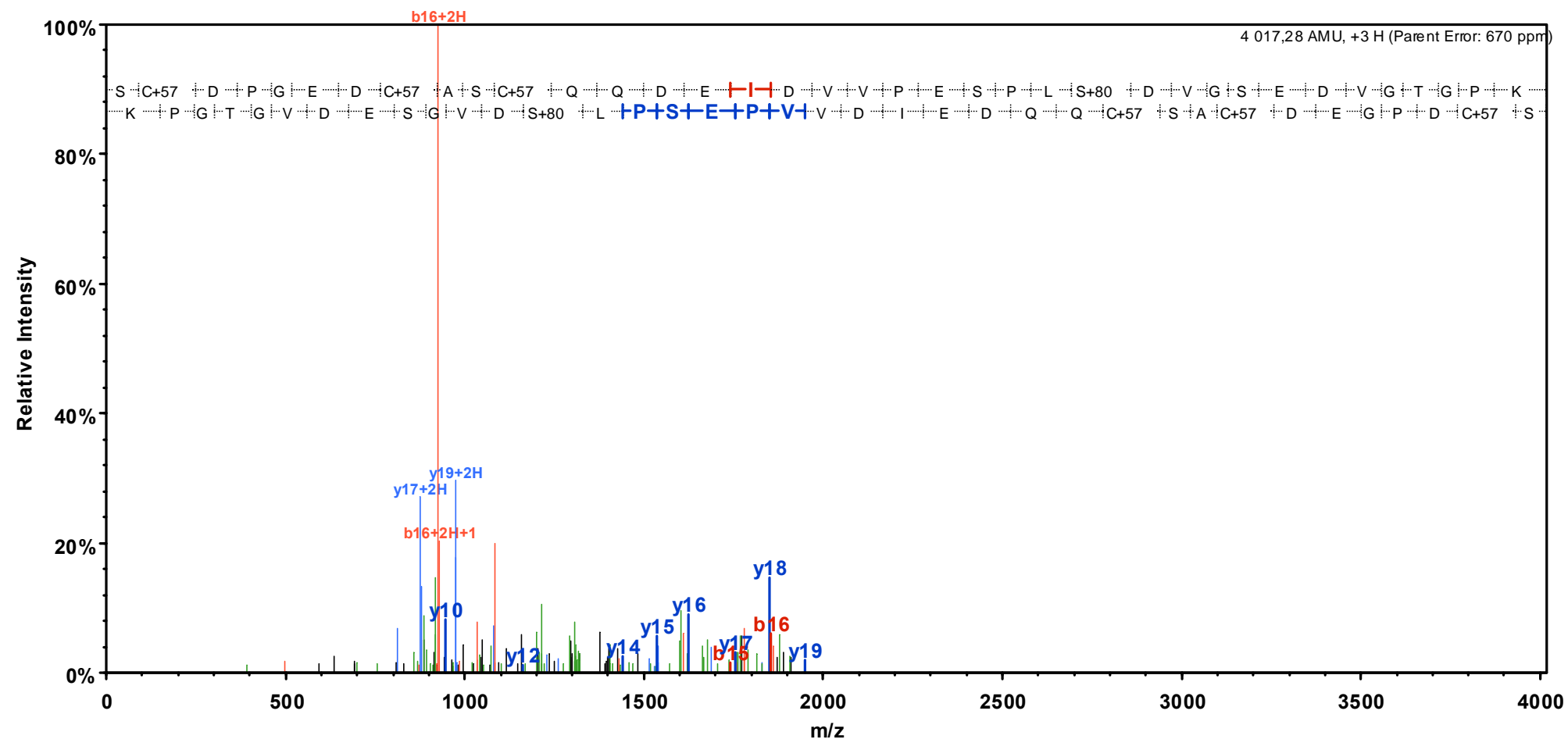

\section{Close to precursor}

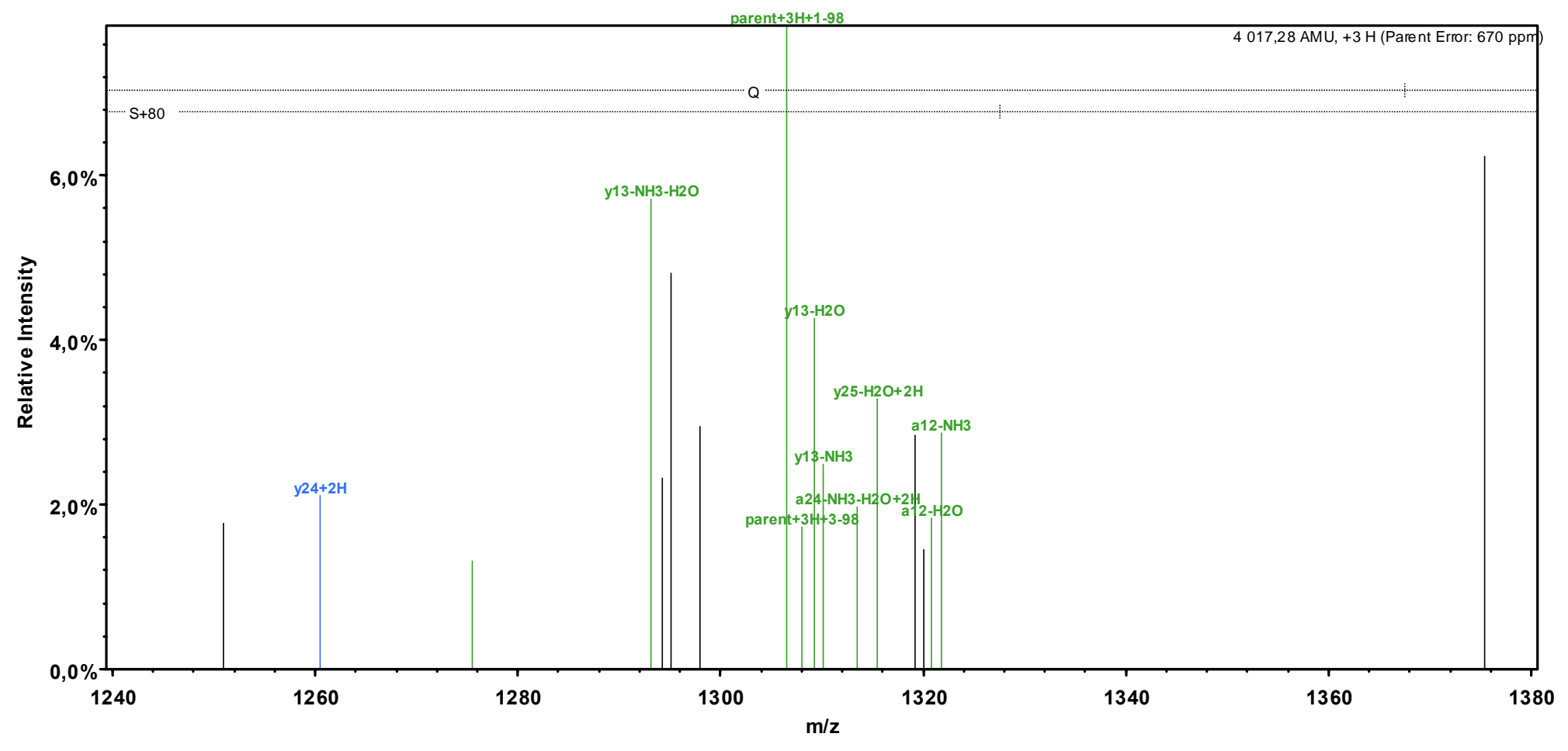




\section{Left of precursor}

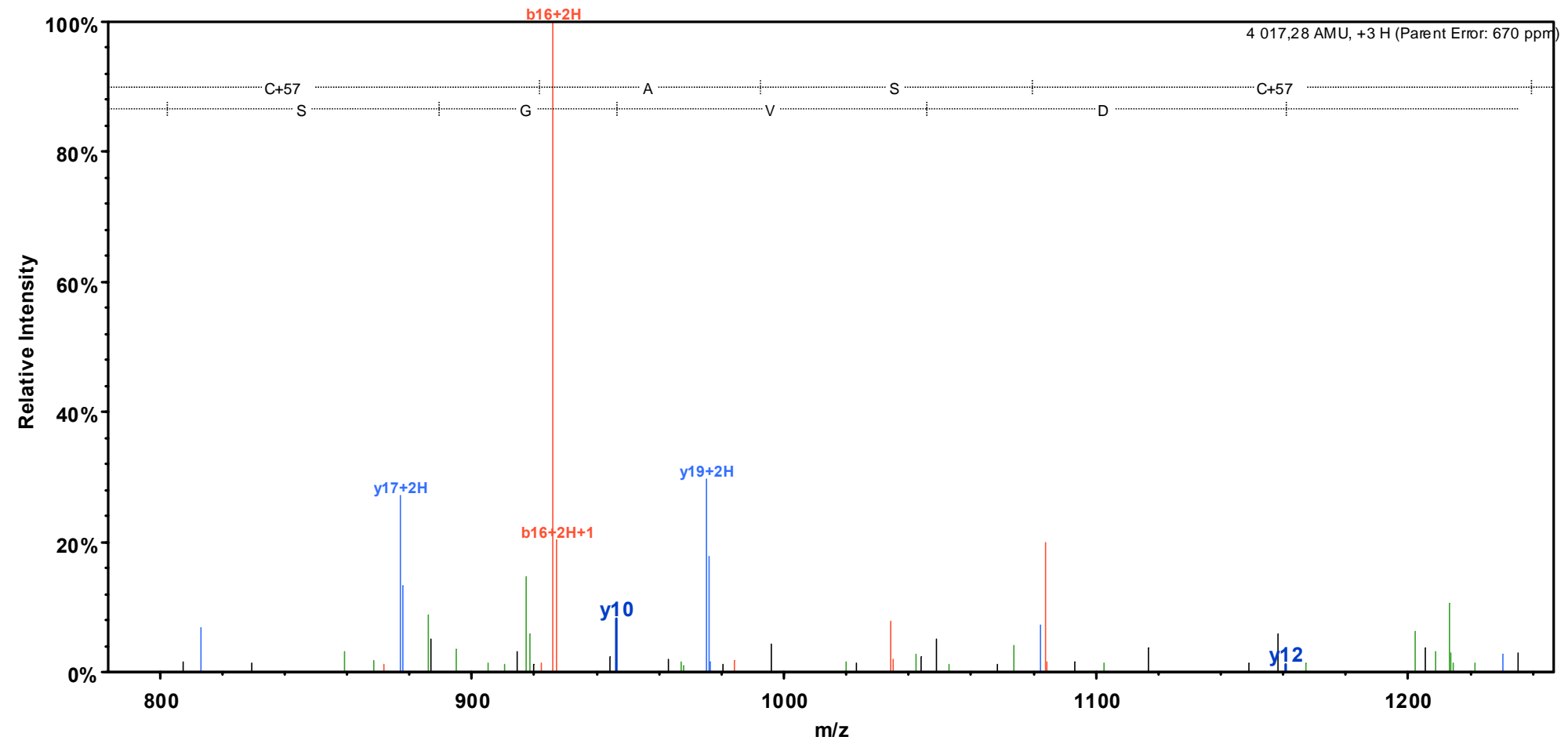

\section{$\underline{\text { Right of precursor }}$}

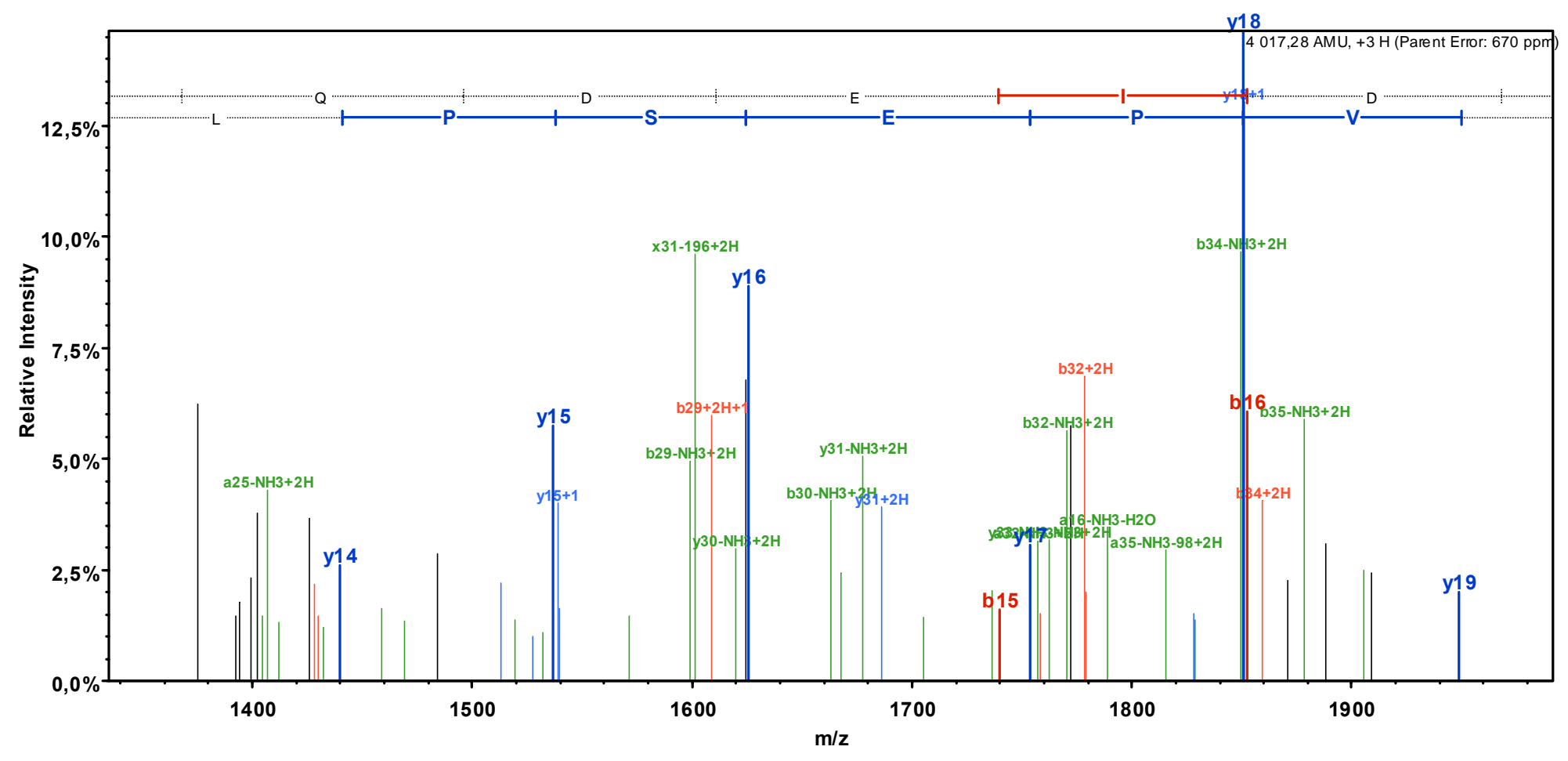




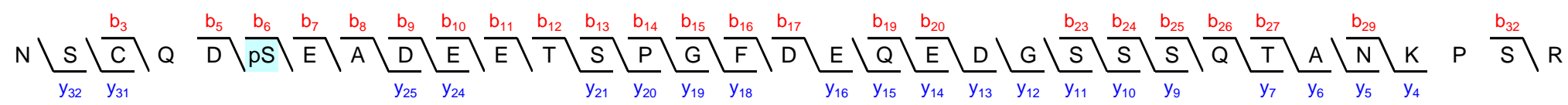

Sequest: XCorr $=6.29, \mathrm{dCn}=0.14$.

Ascore $=23.35$.

Phosphorylation site is localized with $\geq 99 \%$ certainty as Ascore $\geq 20$ (Nat Biotechnol. 2006;24(10):1285-92).

\section{MS/MS spectrum's fragmentation table}

\begin{tabular}{|c|c|c|c|c|c|c|c|c|c|c|}
\hline B & B Ions & $\mathrm{B}+2 \mathrm{H}$ & $\mathrm{B}-\mathrm{NH} 3$ & $\mathrm{~B}-\mathrm{H} 2 \mathrm{O}$ & AA & $Y$ Ions & $\mathrm{Y}+2 \mathrm{H}$ & $\mathrm{Y}-\mathrm{NH} 3$ & $\mathrm{Y}-\mathrm{H} 2 \mathrm{O}$ & $Y$ \\
\hline 1 & 115,1 & & 98,0 & & $\mathrm{~N}$ & 3669,4 & 1835,2 & 3652,4 & 3651,4 & 33 \\
\hline 2 & 202,1 & & 185,1 & 184,1 & $S$ & 3555,4 & 1778.2 & 3538,3 & 3537,4 & 32 \\
\hline 3 & 362.1 & & 345,1 & 344,1 & $C+57$ & 3468,3 & 1734.7 & 3451,3 & 3450,3 & 31 \\
\hline 4 & 490,2 & & 473.1 & 472,2 & $\mathbf{Q}$ & 3308,3 & 1654.7 & 3291,3 & 3290,3 & 30 \\
\hline 5 & 605.2 & & 588.2 & 587,2 & D & $3 \mathbf{1 8 0 , 2}$ & 1590,6 & 3163,2 & 3162,2 & 29 \\
\hline 6 & 772.2 & 386,6 & 755.2 & 754,2 & $\mathbf{S + 8 0}$ & 3065,2 & 1533,1 & 3048,2 & 3047,2 & 28 \\
\hline 7 & 901,2 & 451,1 & 884,2 & 883,2 & E & 2898,2 & 1449,6 & 2881,2 & 2880,2 & 27 \\
\hline 8 & 972.3 & 486,6 & 955,3 & 954.3 & A & 2769,2 & 1385,1 & 2752,1 & 2751,2 & 26 \\
\hline 9 & 1087.3 & 544,2 & 1070,3 & 1069,3 & D & 2698,1 & 1349.6 & 2681,1 & 2680,1 & 25 \\
\hline 10 & 1216,3 & 608,7 & 1199.3 & 1198.3 & $E$ & 2583.1 & 1292.1 & 2566.1 & 2565,1 & 24 \\
\hline 11 & 1345.4 & 673,2 & 1328.4 & 1327.4 & $E$ & 2454,1 & 1227.5 & 2437,0 & 2436,1 & 23 \\
\hline 12 & 1446.4 & 723,7 & 1429.4 & 1428.4 & $T$ & 2325,0 & 1163,0 & 2308,0 & 2307,0 & 22 \\
\hline 13 & 1533.5 & 767.2 & 1516.4 & 1515.5 & S & 2224,0 & 1112.5 & 2206.9 & 2206,0 & 21 \\
\hline 14 & 1630.5 & $\mathbf{8 1 5 , 8}$ & 1613.5 & 1612,5 & $\mathbf{P}$ & 2136,9 & 1069.0 & 2119,9 & 8,9 & 20 \\
\hline 15 & 1687.5 & 844.3 & 1670,5 & 1669,5 & G & 2039,9 & 1020.4 & 2022,9 & 1,9 & 19 \\
\hline 16 & 1834.6 & 917.8 & 1817.6 & 1816.6 & $F$ & 1982,9 & 991.9 & 1965,8 & 1964,9 & 18 \\
\hline 17 & 1949.6 & 975,3 & 1932,6 & 1931,6 & D & 1835,8 & 918,4 & 1818,8 & 1817,8 & 17 \\
\hline 18 & 2078,7 & 1039,8 & 2061,7 & 2060,7 & $E$ & 1720,8 & 860.9 & 1703.7 & 1702,8 & 16 \\
\hline 19 & 2206,7 & 1103.9 & 2189,7 & 2188.7 & $\mathbf{Q}$ & 1591.7 & 796.4 & 1574.7 & 1573.7 & 15 \\
\hline 20 & 2335,8 & 1168.4 & 2318,8 & 2317,8 & $E$ & 1463.7 & 732.3 & 1446.6 & 1445,7 & 14 \\
\hline 21 & 2450,8 & 1225,9 & 2433,8 & 2432.8 & D & 1334.6 & 667.8 & 1317,6 & 1316.6 & 13 \\
\hline 22 & 2507,8 & 1254,4 & 2490,8 & 2489,8 & G & 1219,6 & 610.3 & 1202.6 & 1201,6 & 12 \\
\hline 23 & 2594,9 & 1297.9 & 2577,8 & 2576,9 & S & 1162.6 & 581.8 & 1145,6 & 1144,6 & 11 \\
\hline 24 & 2681,9 & 1341.5 & 2664,9 & 2663,9 & S & 1075.5 & 538.3 & 1058.5 & 1057,5 & 10 \\
\hline 25 & 2768,9 & 1385.0 & 2751,9 & 2750,9 & S & 988.5 & 494,8 & 971,5 & 970.5 & 9 \\
\hline 26 & 2897,0 & 1449.0 & 2880,0 & 2879,0 & $\mathbf{Q}$ & 901,5 & 451,2 & 884.5 & 883,5 & 8 \\
\hline 27 & 2998,0 & 1499.5 & 2981,0 & 2980,0 & $\mathbf{T}$ & 773.4 & 387,2 & 756,4 & 755,4 & 7 \\
\hline 28 & 3069,1 & 1535,0 & 3052,0 & 3051,1 & A & 672.4 & 336,7 & 655.4 & 654,4 & 6 \\
\hline 29 & 3183.1 & 1592.1 & 3166.1 & 3165,1 & $\mathrm{~N}$ & 601.3 & 301,2 & 584,3 & 583,3 & 5 \\
\hline 30 & 3311,2 & 1656.1 & 3294,2 & 3293,2 & K & 487.3 & 244,2 & 470,3 & 469,3 & 4 \\
\hline 31 & 3408,3 & 1704,6 & 3391,2 & 3390,3 & $\mathbf{P}$ & 359,2 & & 342,2 & 341,2 & 3 \\
\hline 32 & 3495,3 & 1748.2 & 3478,3 & 3477,3 & S & 262,2 & & 245,1 & 244,1 & 2 \\
\hline 33 & 3669,4 & 1835,2 & 3652,4 & 3651,4 & $\mathbf{R}$ & 175,1 & & 158,1 & & 1 \\
\hline
\end{tabular}




\section{MS/MS spectrum}

\section{Full-range}

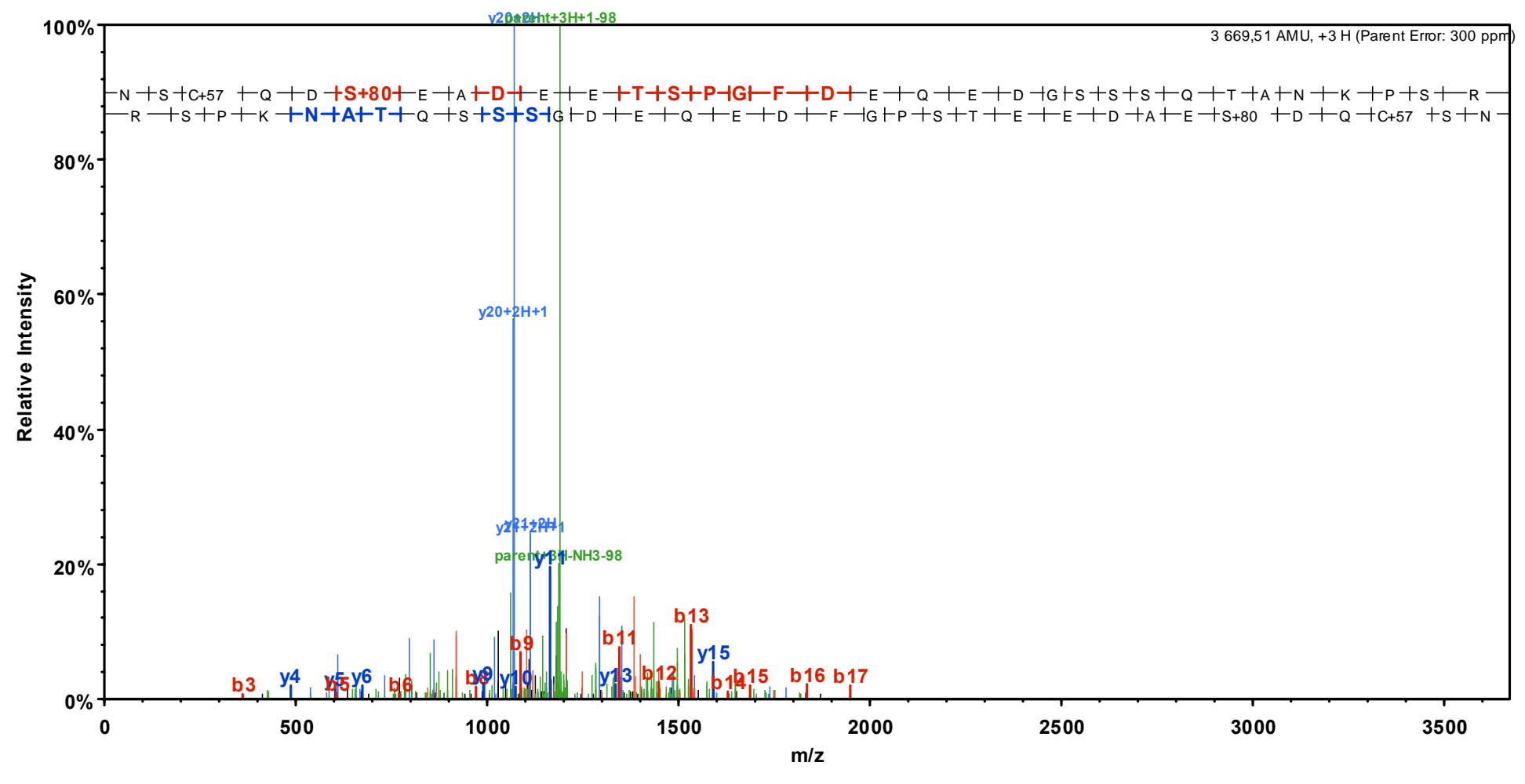

\section{$\underline{\text { Close to precursor }}$}

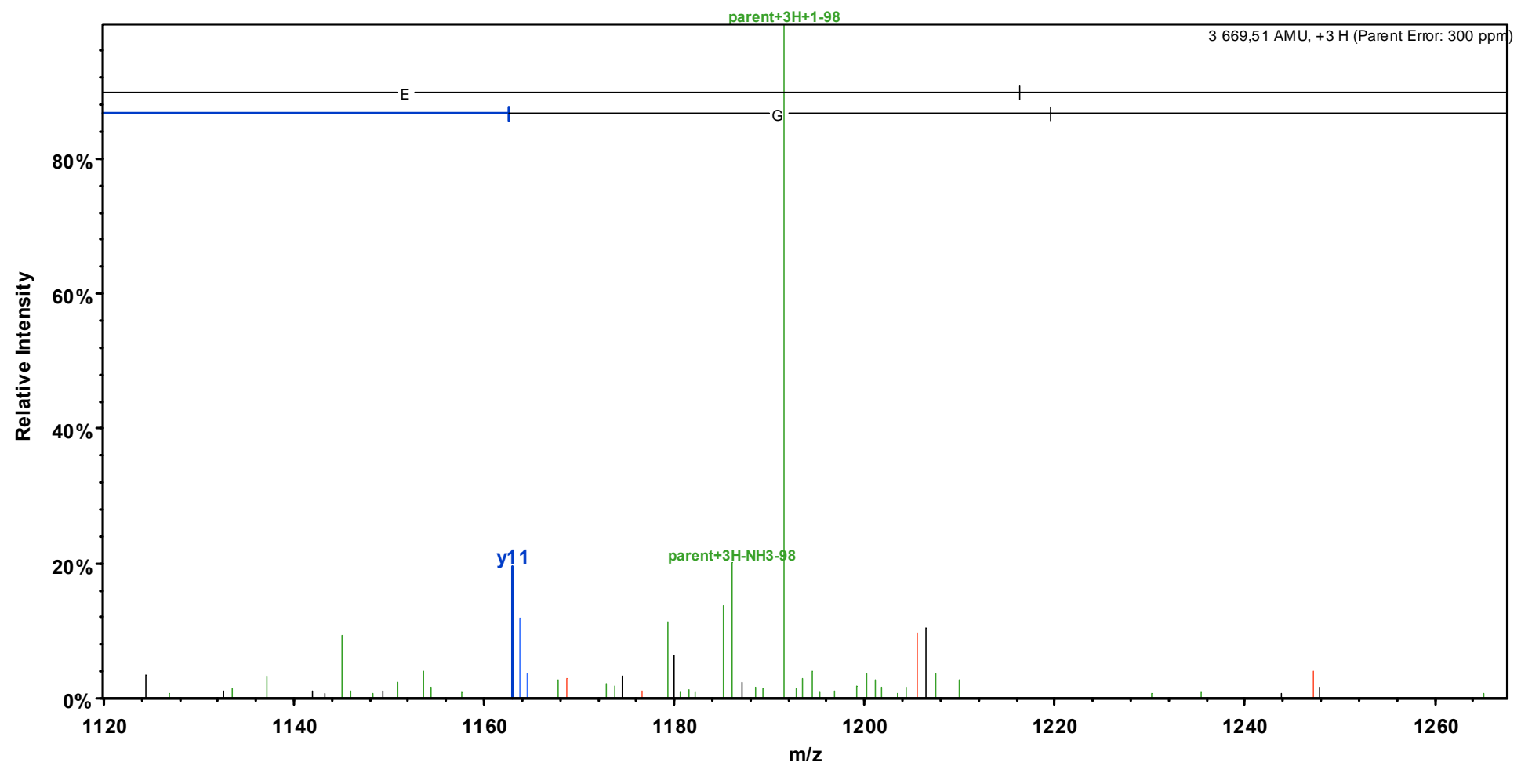




\section{Left of precursor}

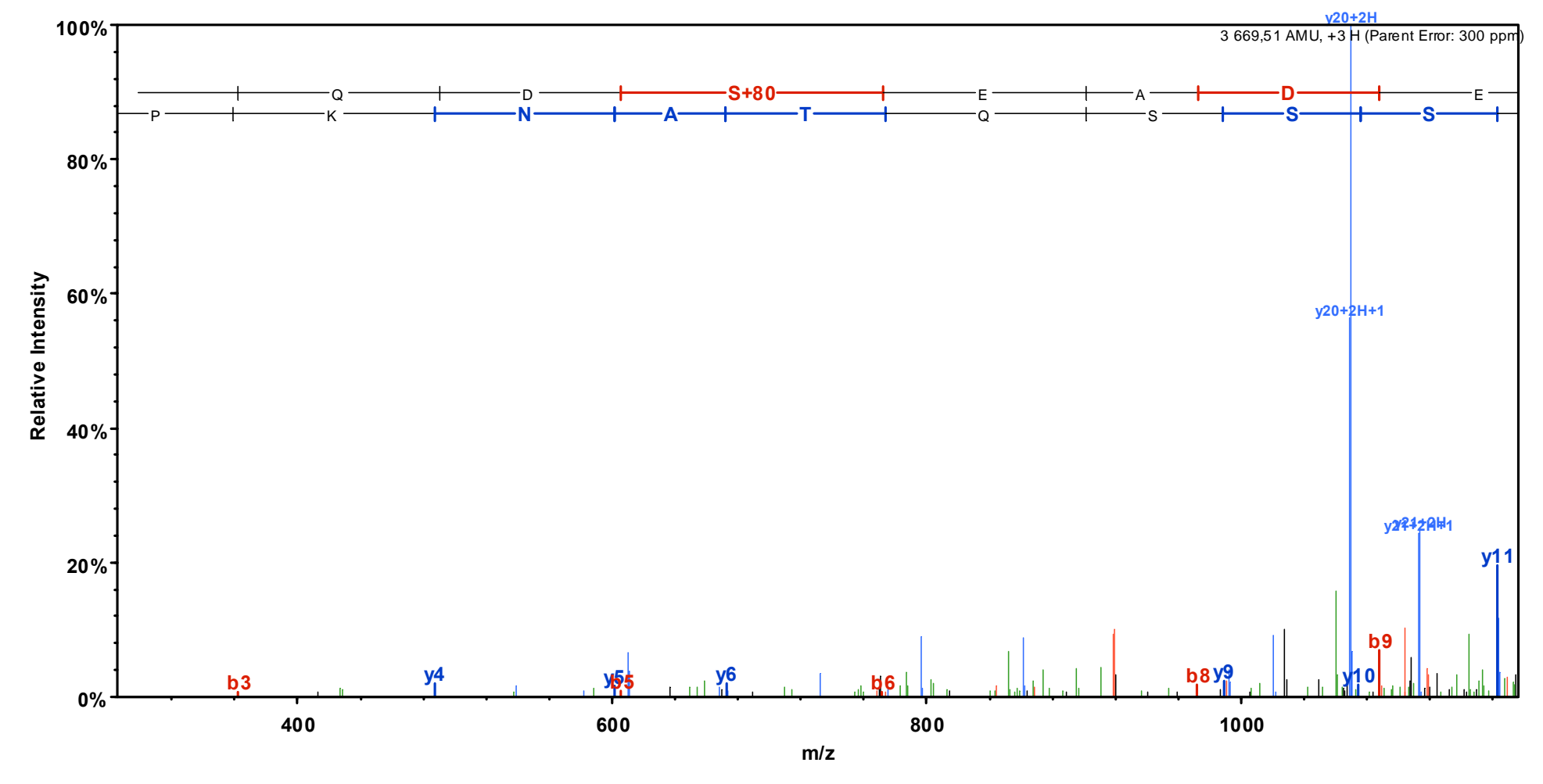

\section{$\underline{\text { Right of precursor }}$}

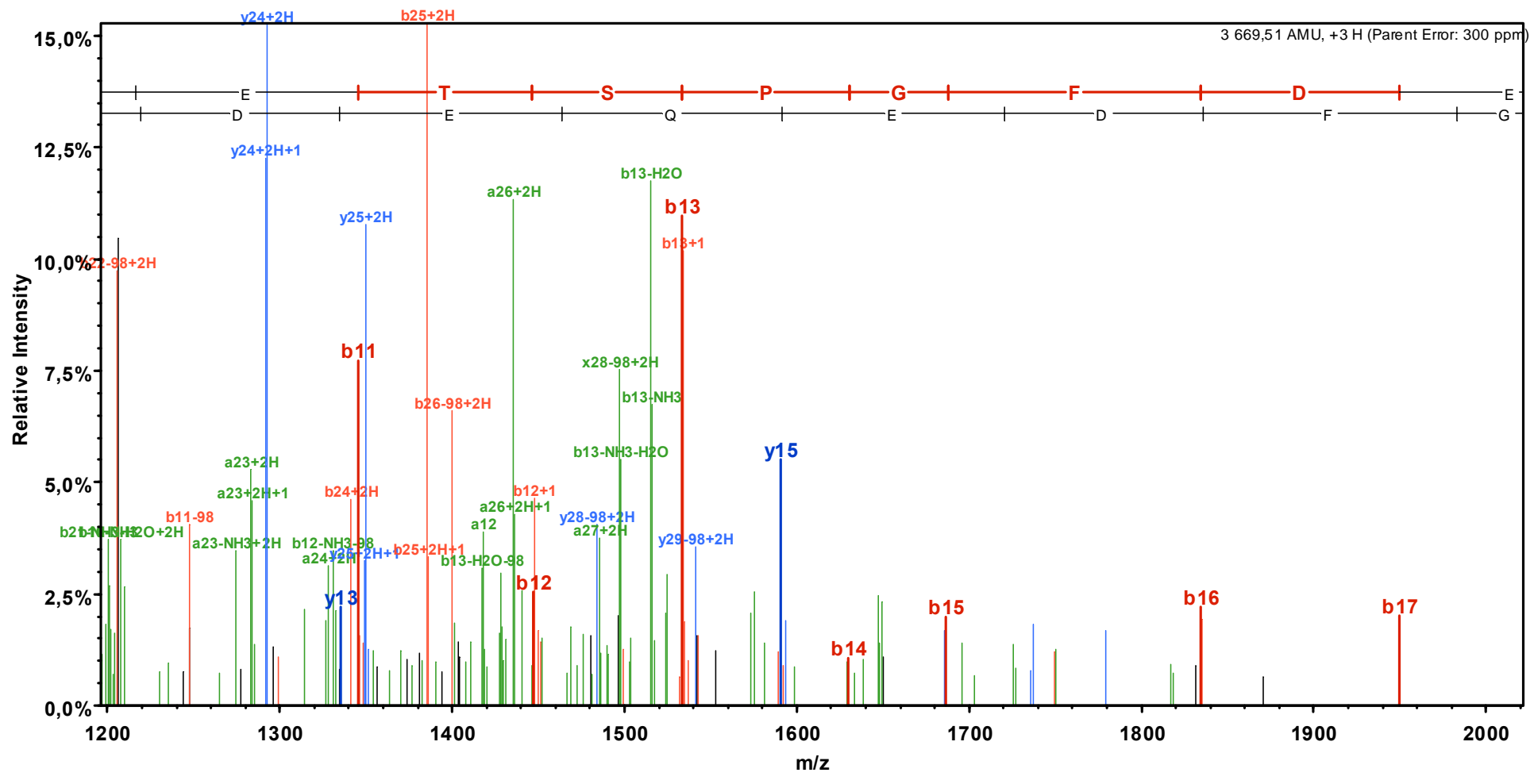




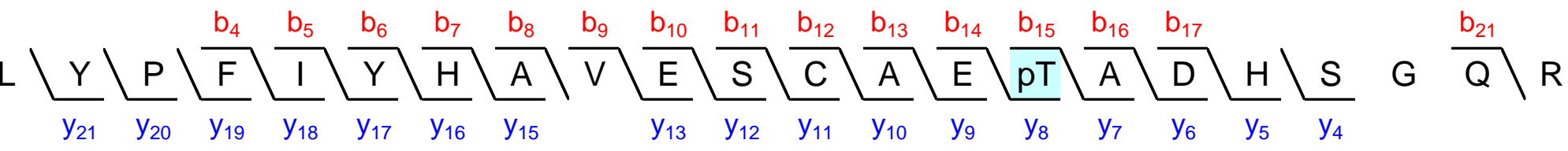

Sequest: $\mathrm{XCorr}=3.97, \mathrm{dCn} 1=0.05$ (between $1^{\text {st }}$ and $2^{\text {nd }}$ best matches), $\mathrm{dCn} 2=0.10$ (between $2^{\text {nd }}$ and $3^{\text {rd }}$ best matches).

Ascore $=7.65$.

Sequest's second best match with XCorr $=3.76$ is to the same peptide sequence but with an alternative phosphorylation site: LYPFIYHAVEpSCAEpTADHSGQR.

The weak Ascore indicates that, given the observed MS/MS spectrum, the phosphopeptide identified could either be Sequest's first or second best match (Nat Biotechnol. 2006;24(10):1285-92).

\section{MS/MS spectrum's fragmentation table}

\begin{tabular}{|c|c|c|c|c|c|c|c|c|c|c|}
\hline B & B Ions & $\mathrm{B}+2 \mathrm{H}$ & $\mathrm{B}-\mathrm{NH} 3$ & $\mathrm{~B}-\mathrm{H} 2 \mathrm{O}$ & AA & Y Ions & $\mathrm{Y}+2 \mathrm{H}$ & $\mathrm{Y}-\mathrm{NH} 3$ & $\mathrm{Y}-\mathrm{H} 2 \mathrm{O}$ & $Y$ \\
\hline 1 & 114,1 & & & & $\mathbf{L}$ & 2631,1 & 1316,1 & 2614,1 & 2613,1 & 22 \\
\hline 2 & 277,2 & & & & $\mathrm{Y}$ & 2518,0 & 1259.5 & 2501,0 & 2500,0 & 21 \\
\hline 3 & 374,2 & & & & $\mathbf{P}$ & 2355,0 & 1178.0 & 2338,0 & 2337,0 & 20 \\
\hline 4 & 521.3 & & & & $\mathbf{F}$ & 2257,9 & 1129.5 & 2240,9 & 2239,9 & 19 \\
\hline 5 & 634.4 & & & & I & 2110,9 & 1055.9 & 2093,8 & 2092,9 & 18 \\
\hline 6 & 797.4 & 399,2 & & & $\mathrm{Y}$ & 1997,8 & 999.4 & 1980,8 & 1979,8 & 17 \\
\hline 7 & 934.5 & 467,7 & & & H & 1834,7 & 917.9 & 1817,7 & 1816,7 & 16 \\
\hline 8 & 1005.5 & 503.3 & & & A & 1697,7 & 849.3 & 1680,6 & 1679,6 & 15 \\
\hline 9 & 1104.6 & 552,8 & & & V & 1626.6 & 813,8 & 1609.6 & 1608,6 & 14 \\
\hline 10 & 1233.6 & 617.3 & & 1215,6 & E & 1527,6 & 764.3 & 1510.5 & 1509.5 & 13 \\
\hline 11 & 1320.7 & 660.8 & & 1302,7 & S & 1398.5 & 699,8 & 1381.5 & 1380,5 & 12 \\
\hline 12 & 1480,7 & 740.9 & & 1462,7 & $C+57$ & 1311,5 & 656.2 & 1294,5 & 1293,5 & 11 \\
\hline 13 & 1551,7 & 776.4 & & 1533,7 & A & 1151,4 & 576,2 & 1134.4 & 1133.4 & 10 \\
\hline 14 & 1680,8 & 840.9 & & 1662,8 & E & 1080.4 & 540,7 & 1063.4 & 1062,4 & 9 \\
\hline 15 & 1861,8 & 931.4 & & 1843,8 & $\mathbf{T}+\mathbf{8 0}$ & 951.4 & 476,2 & 934.3 & 933.4 & 8 \\
\hline 16 & 1932,8 & 966.9 & & 1914,8 & A & 770.4 & 385,7 & 753.3 & 752,3 & 7 \\
\hline 17 & 2047,9 & 1024.4 & & 2029,8 & D & 699.3 & 350.2 & 682.3 & 681,3 & 6 \\
\hline 18 & 2184,9 & 1093,0 & & 2166,9 & H & 584.3 & 292.6 & 567.3 & 566,3 & 5 \\
\hline 19 & 2271,9 & 1136,5 & & 2253,9 & S & 447,2 & & 430.2 & 429.2 & 4 \\
\hline 20 & 2329,0 & 1165,0 & & 2311,0 & G & 360,2 & & 343.2 & & 3 \\
\hline 21 & 2457,0 & 1229.0 & 2440,0 & 2439,0 & $\mathbf{Q}$ & 303,2 & & 286.2 & & 2 \\
\hline 22 & 2631,1 & 1316,1 & 2614.1 & 2613,1 & R & 175,1 & & 158,1 & & 1 \\
\hline
\end{tabular}




\section{MS/MS spectrum}

\section{Full-range}

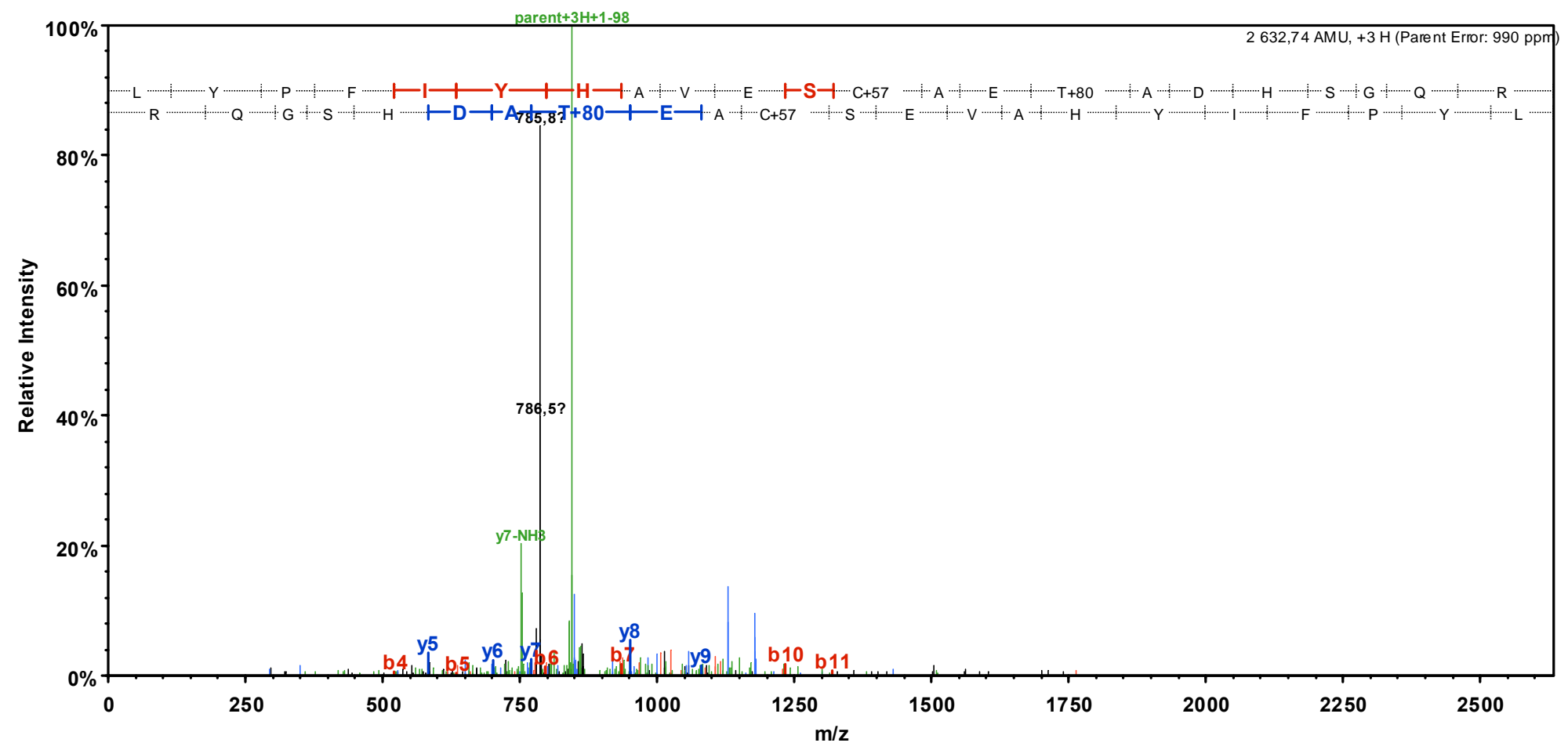

\section{Close to precursor}

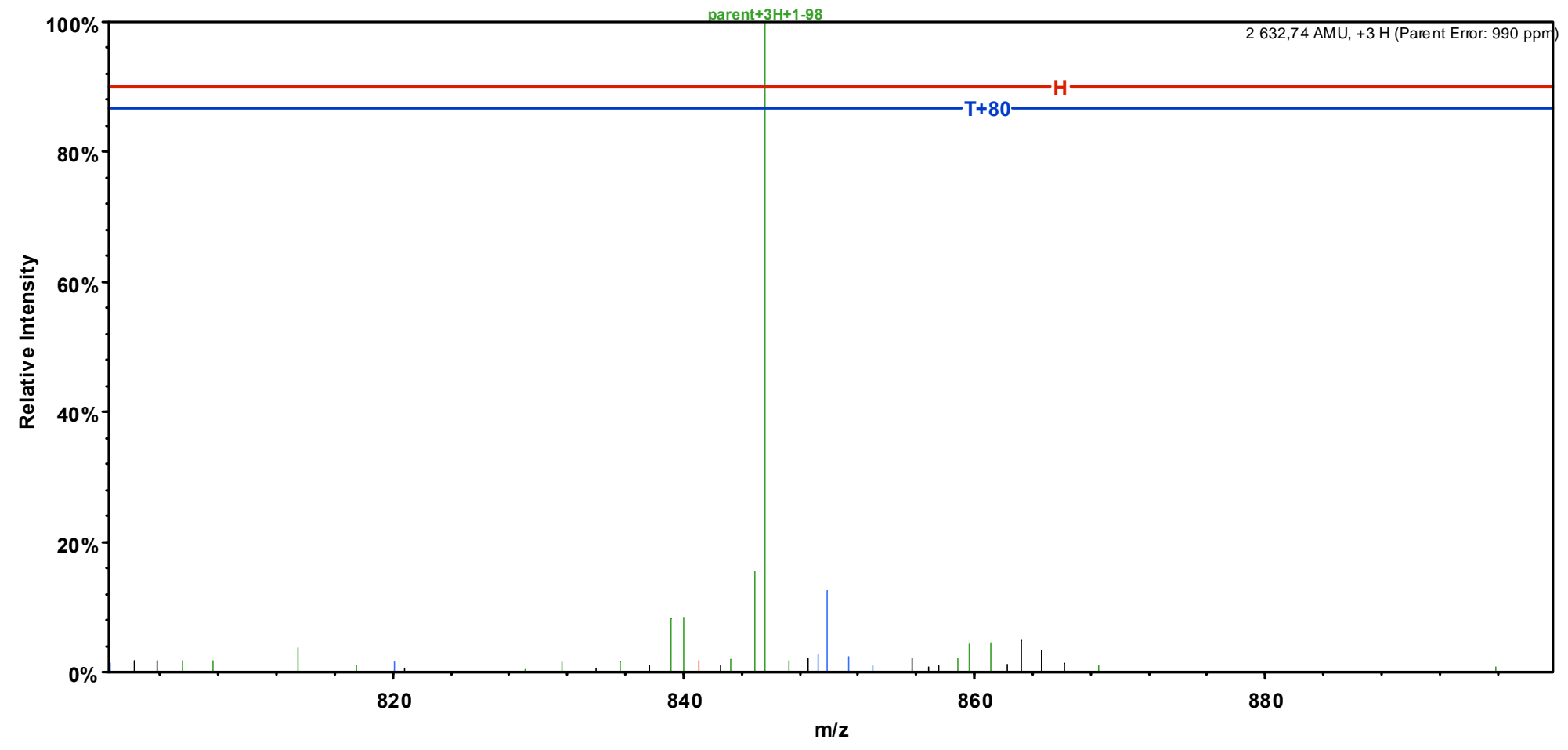




\section{Left of precursor}

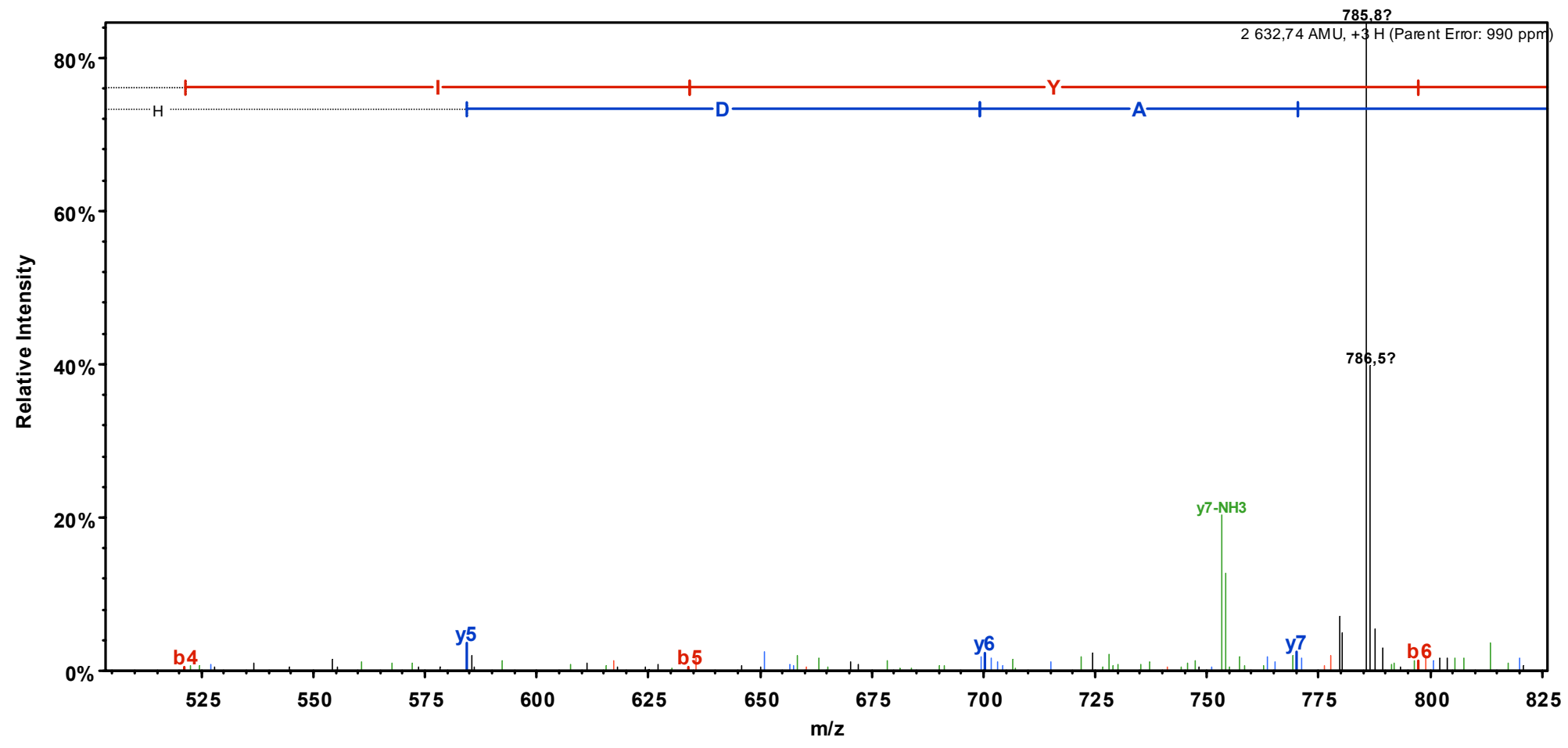

\section{$\underline{\text { Right of precursor }}$}

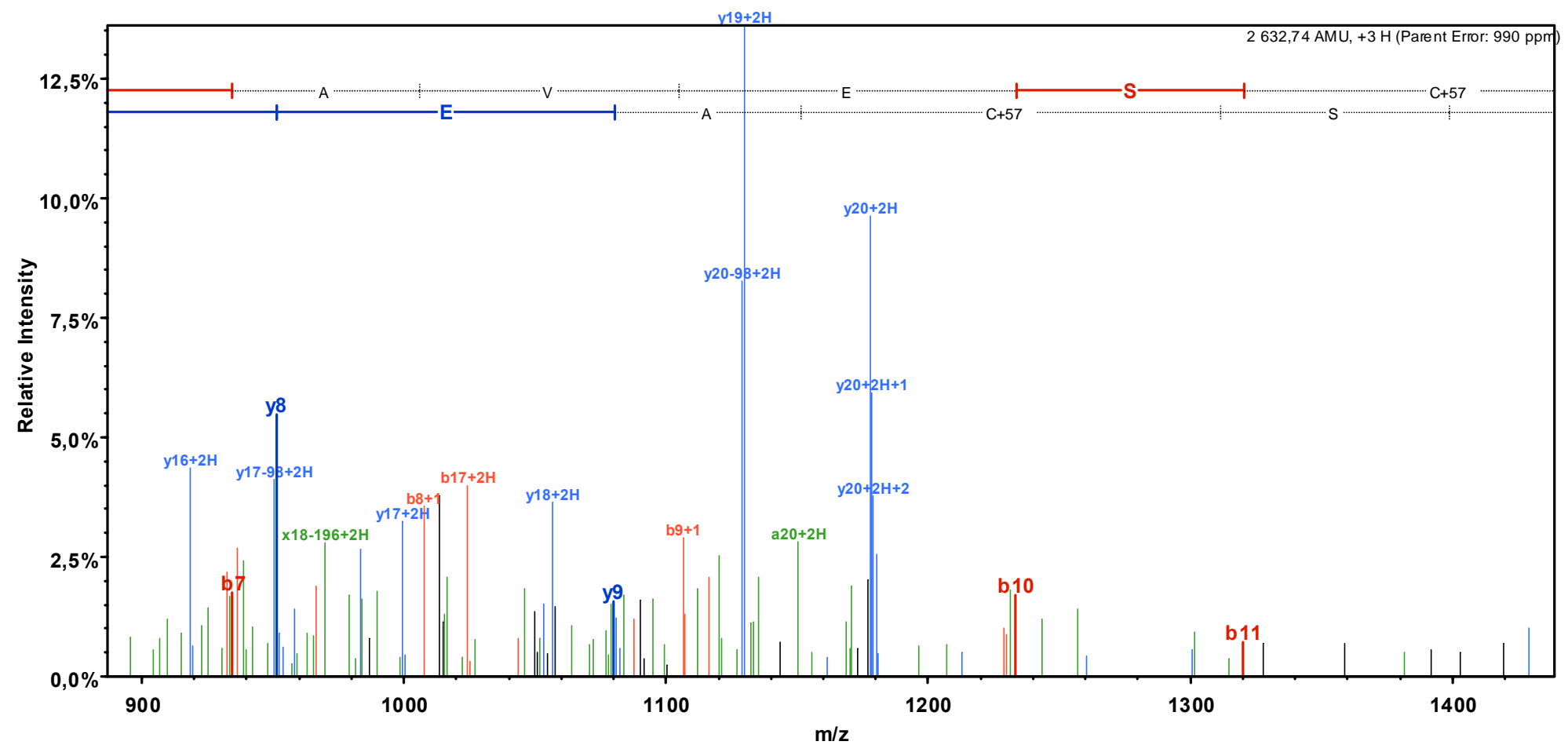




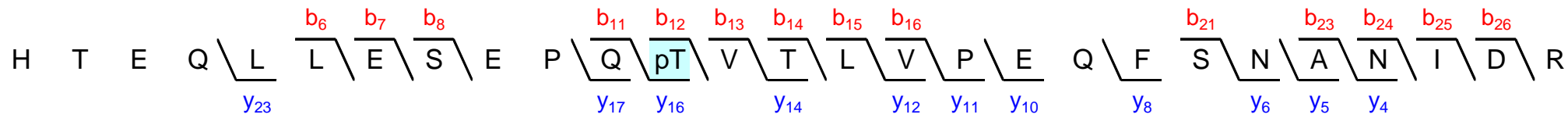

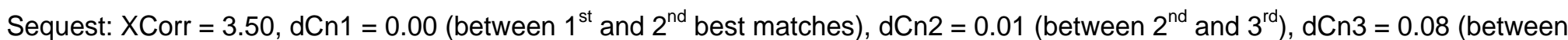
$3^{\text {rd }}$ and $\left.4^{\text {th }}\right)$.

Ascore $=5.06$.

Sequest's second and third best matches with XCorr $=3.50$ and XCorr $=3.25$ respectively are to the same peptide sequence but with alternative phosphorylation sites:

HTEQLLEPSEPQTVTLVPEQFSNANIDR

HPTEQLLESEPQTVTLVPEQFSNANIDR

The weak Ascore indicates that, given the observed MS/MS spectrum, the phosphopeptide identified could either be Sequest's first, second or third best match (Nat Biotechnol. 2006;24(10):1285-92).

\section{MS/MS spectrum's fragmentation table}

\begin{tabular}{|c|c|c|c|c|c|c|c|c|c|c|}
\hline B & B Ions & $\mathrm{B}+2 \mathrm{H}$ & $\mathrm{B}-\mathrm{NH} 3$ & $\mathrm{~B}-\mathrm{H} 2 \mathrm{O}$ & AA & $Y$ Ions & $\mathrm{Y}+2 \mathrm{H}$ & $\mathrm{Y}-\mathrm{NH} 3$ & $\mathrm{Y}-\mathrm{H} 2 \mathrm{O}$ & $Y$ \\
\hline 1 & 138.1 & 69,5 & & & $\mathrm{H}$ & 3175,5 & 1588,3 & 3158,5 & 3157,5 & 27 \\
\hline 2 & 239.1 & 120.1 & & 221,1 & $\mathbf{T}$ & 3038,4 & 1519,7 & 3021,4 & 3020,4 & 26 \\
\hline 3 & 368,2 & 184,6 & & 350,1 & E & 2937.4 & 1469,2 & 2920,4 & 2919,4 & 25 \\
\hline 4 & 496.2 & 248,6 & 479,2 & 478,2 & $\mathbf{Q}$ & 2808,3 & 1404,7 & 2791,3 & 2790,3 & 24 \\
\hline 5 & 609,3 & 305,2 & 592,3 & 591,3 & L & 2680,3 & 1340.6 & 2663,3 & 2662,3 & 23 \\
\hline 6 & 722.4 & 361,7 & 705.4 & 704,4 & L & 2567,2 & 1284,1 & 2550,2 & 2549,2 & 22 \\
\hline 7 & 851.4 & 426.2 & 834.4 & 833.4 & E & 2454,1 & 1227,6 & 2437,1 & 2436,1 & 21 \\
\hline 8 & 938.5 & 469,7 & 921.4 & 920,4 & S & 2325,1 & 1163,0 & 2308,0 & 2307,1 & 20 \\
\hline 9 & 1067,5 & 534,3 & 1050,5 & 1049,5 & E & 2238,0 & 1119,5 & 2221,0 & 2220,0 & 19 \\
\hline 10 & 1164,6 & 582,8 & 1147,5 & 1146,5 & $\mathbf{P}$ & 2109,0 & 1055,0 & 2092,0 & 2091,0 & 18 \\
\hline 11 & 1292.6 & 646.8 & 1275.6 & 1274,6 & $\mathbf{Q}$ & 2011,9 & 1006.5 & 1994,9 & 1993,9 & 17 \\
\hline 12 & 1473,6 & 737.3 & 1456,6 & 1455.6 & $T+80$ & 1883,9 & 942.4 & 1866,9 & 1865,9 & 16 \\
\hline 13 & 1572.7 & 786.9 & 1555,7 & 1554.7 & V & 1702,9 & 851,9 & 1685,8 & 1684,9 & 15 \\
\hline 14 & 1673,7 & 837.4 & 1656,7 & 1655,7 & $\mathbf{T}$ & 1603.8 & 802.4 & 1586,8 & 1585,8 & 14 \\
\hline 15 & 1786.8 & 893.9 & 1769,8 & 1768,8 & L & 1502,8 & 751,9 & 1485,7 & 1484,7 & 13 \\
\hline 16 & 1885.9 & 943.5 & 1868,9 & 1867,9 & V & 1389.7 & 695,3 & 1372,6 & 1371.7 & 12 \\
\hline 17 & 1982,9 & 992,0 & 1965,9 & 1964,9 & $\mathbf{P}$ & 1290.6 & 645.8 & 1273.6 & 1272.6 & 11 \\
\hline 18 & 2112,0 & 1056.5 & 2095,0 & 2094,0 & E & 1193.6 & 597,3 & 1176,5 & 1175,5 & 10 \\
\hline 19 & 2240,0 & 1120,5 & 2223,0 & 2222,0 & $\mathbf{Q}$ & 1064.5 & 532,8 & 1047.5 & 1046.5 & 9 \\
\hline 20 & 2387,1 & 1194,1 & 2370.1 & 2369,1 & $F$ & 936,5 & 468,7 & 919,4 & 918.4 & 8 \\
\hline 21 & 2474,1 & 1237.6 & 2457,1 & 2456.1 & S & 789,4 & 395,2 & 772,4 & 771,4 & 7 \\
\hline 22 & 2588,2 & 1294,6 & 2571,2 & 2570,2 & $\mathrm{~N}$ & 702.4 & 351,7 & 685.3 & 684,3 & 6 \\
\hline 23 & 2659,2 & 1330.1 & 2642,2 & 2641,2 & A & 588.3 & & 571,3 & 570,3 & 5 \\
\hline 24 & 2773,3 & 1387.1 & 2756.2 & 2755,3 & $\mathrm{~N}$ & 517.3 & & 500,2 & 499,3 & 4 \\
\hline 25 & 2886.4 & 1443.7 & 2869,3 & 2868,3 & I & 403,2 & & 386,2 & 385,2 & 3 \\
\hline 26 & 3001,4 & 1501.2 & 2984.4 & 2983.4 & D & 290,1 & & 273,1 & 272,1 & 2 \\
\hline 27 & 3175,5 & 1588.3 & 3158.5 & 3157,5 & $\mathbf{R}$ & 175,1 & & 158,1 & & 1 \\
\hline
\end{tabular}




\section{MS/MS spectrum}

\section{Full-range}

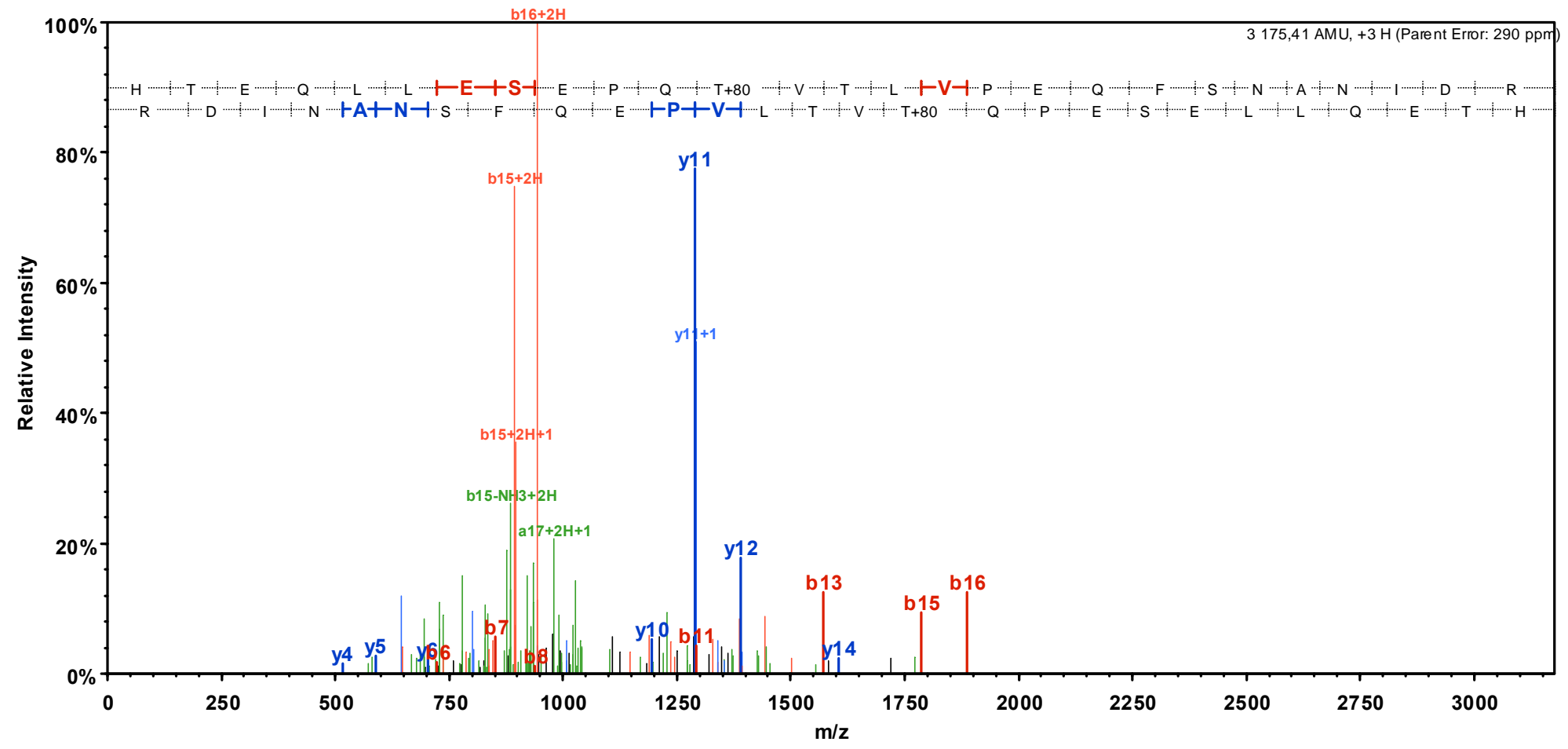

\section{Close to precursor}

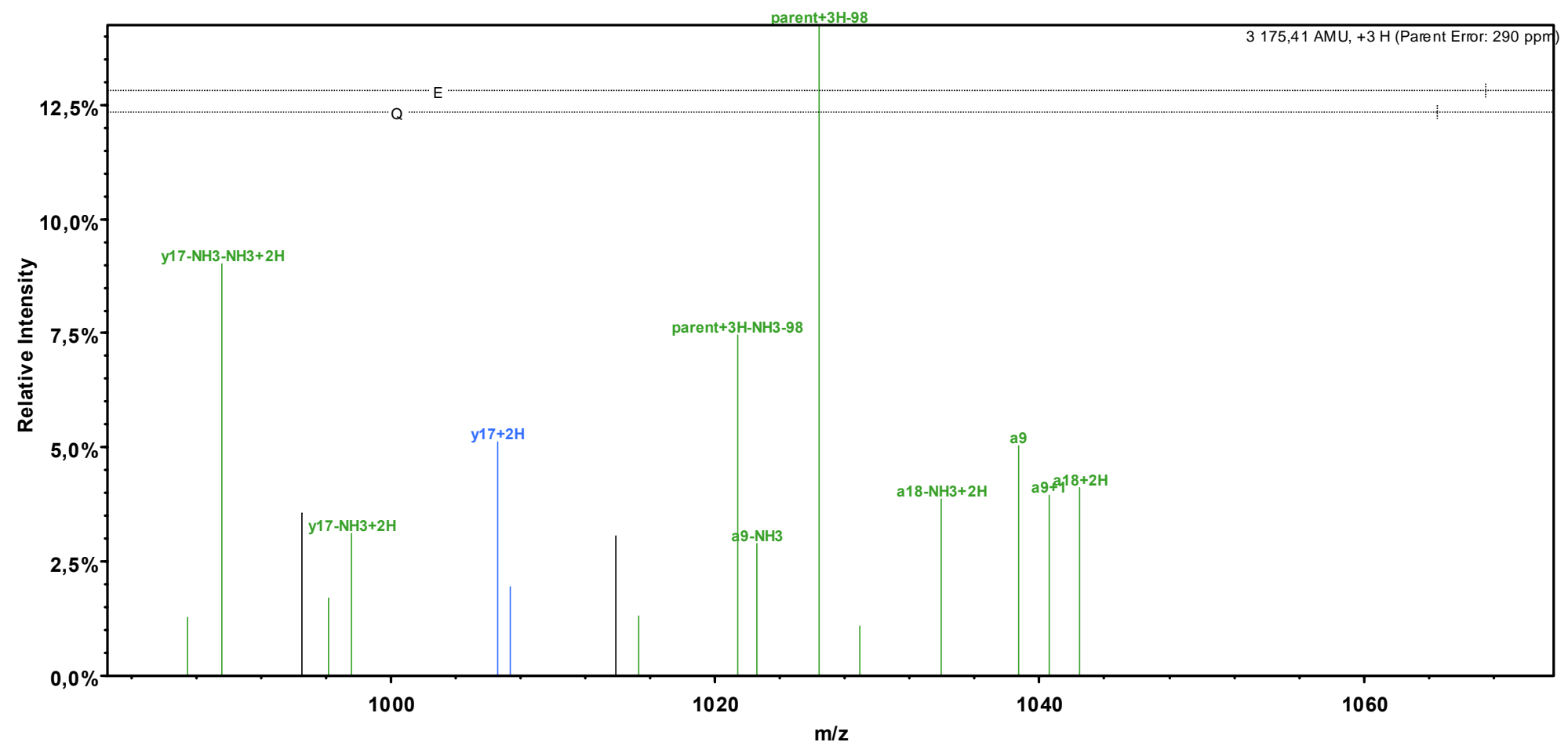




\section{Left of precursor}

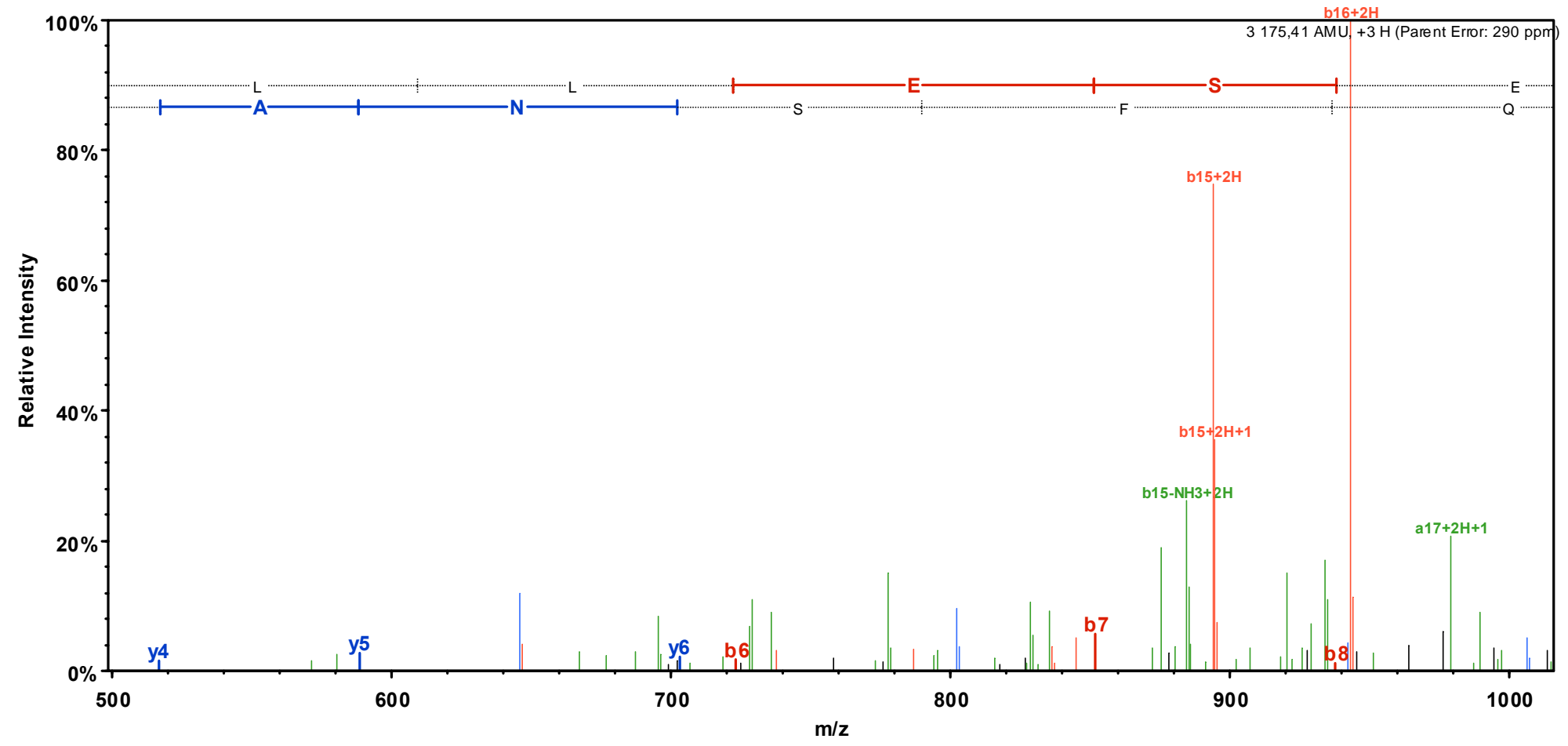

\section{$\underline{\text { Right of precursor }}$}

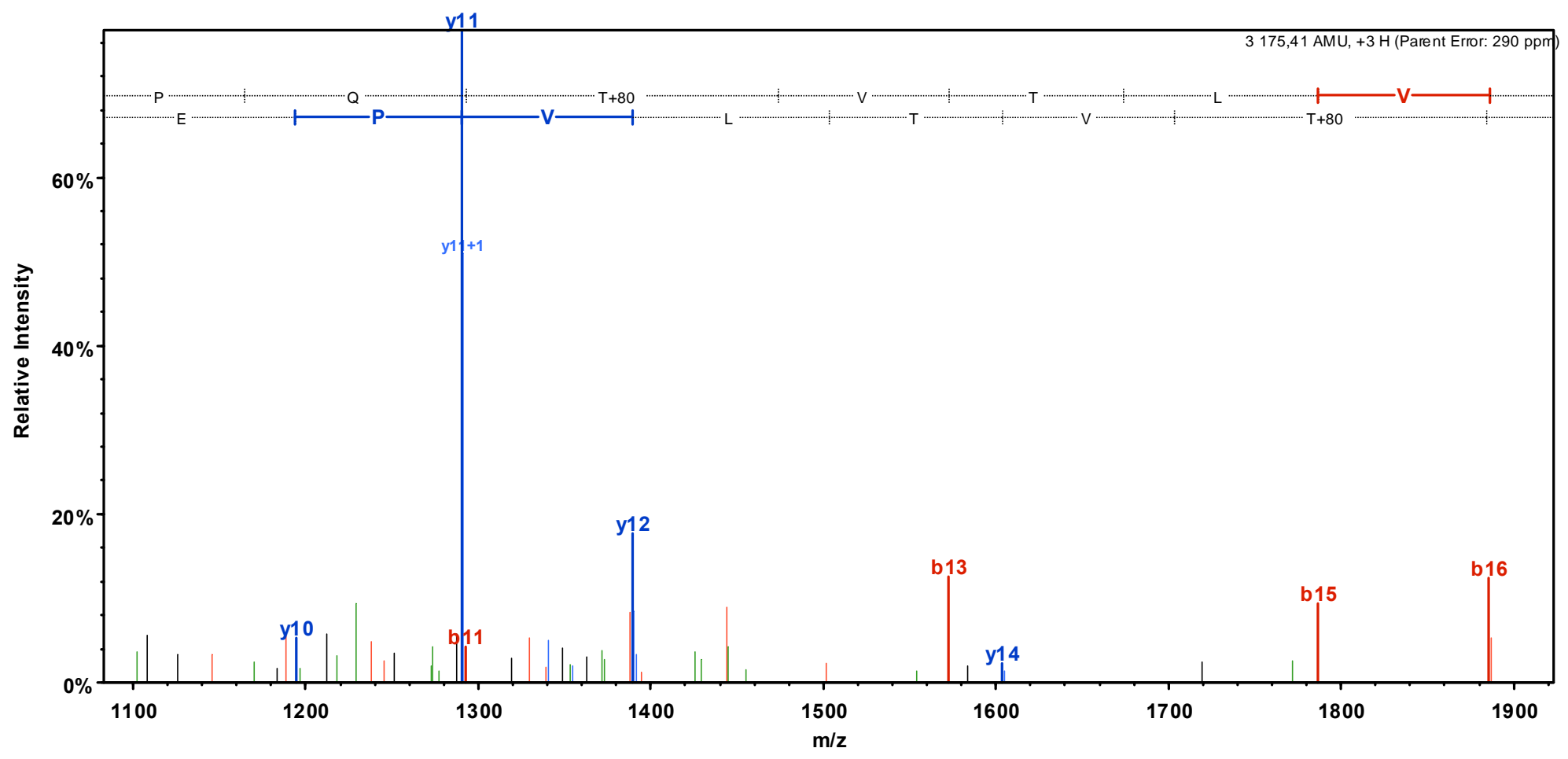

